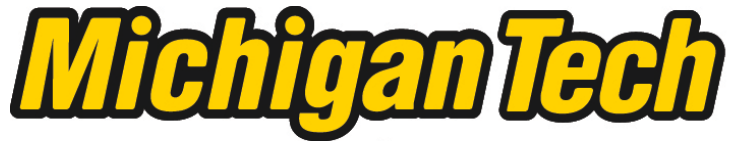 \\ Michigan Technological University Create the Future Digital Commons @ Michigan Tech
}

Integration of computational models and experimental characterization to study internal frost damage in cementitious materials

Kenny $\mathrm{Ng}$

Michigan Technological University

Follow this and additional works at: https://digitalcommons.mtu.edu/etds

Part of the Civil and Environmental Engineering Commons

Copyright 2012 Kenny Ng

\section{Recommended Citation}

$\mathrm{Ng}$, Kenny, "Integration of computational models and experimental characterization to study internal frost damage in cementitious materials ", Dissertation, Michigan Technological University, 2012.

https://doi.org/10.37099/mtu.dc.etds/260

Follow this and additional works at: https://digitalcommons.mtu.edu/etds

3 Part of the Civil and Environmental Engineering Commons 


\title{
INTEGRATION OF COMPUTATIONAL MODELS AND EXPERIMENTAL CHARACTERIZATION TO STUDY INTERNAL FROST DAMAGE IN CEMENTITIOUS MATERIALS
}

By:

Kenny Ng

\begin{abstract}
A DISSERTATION
Submitted in partial fulfillment of the requirements for the degree of DOCTOR OF PHILOSOPHY

In Civil Engineering
\end{abstract}

MICHIGAN TECHNOLOGICAL UNIVERSITY

2012

(C) 2012 Kenny Ng 

This dissertation has been approved in partial fulfillment of the requirements for the Degree of DOCTOR OF PHILOSOPHY in Civil Engineering.

Department of Civil and Environmental Engineering

Dissertation Advisor: Qingli Dai

Committee Member: Zhanping You

Committee Member: Jacob E. Hiller

Committee Member: Ibrahim Miskioglu

Department Chair: $\quad$ David W. Hand 



\section{Table of Contents}

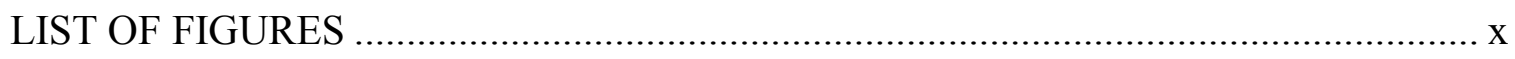

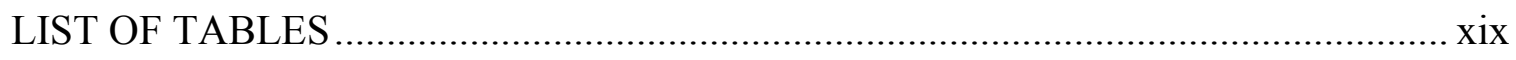

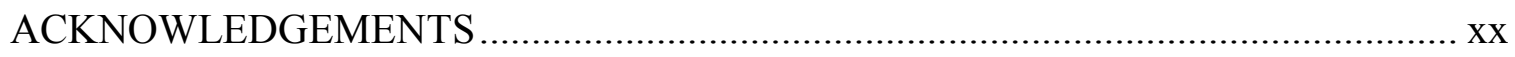

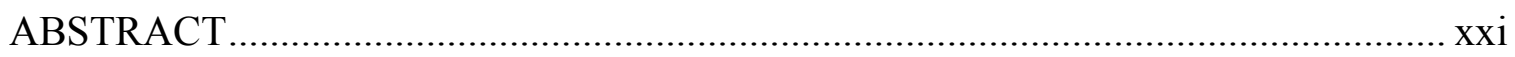

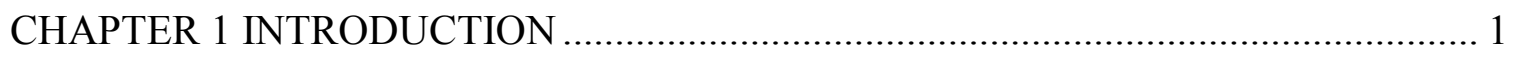

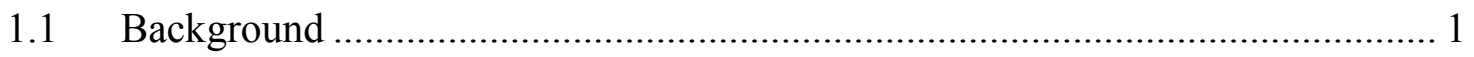

1.2 Literature Review on Concrete Microstructure ................................................ 5

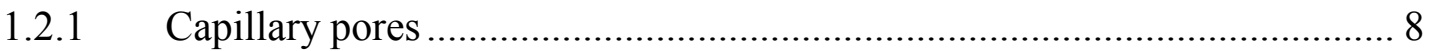

1.3 Literature Review of Internal Frost Damage on Concrete ............................... 9

1.4 Literature Review on Micro-damage Modeling............................................ 15

1.4.1 Micro-mechanical modeling of damage behavior ................................... 15

1.4.2 Extended Finite Element Method (XFEM) for fracture behavior .............. 17

1.4.3 Cohesive zone modeling (CZM) for fracture behavior.......................... 18

1.5 Literature Review on Image Acquisition and Processing Techniques............. 21

1.5.1 Scanning electron microscope (SEM) on capturing 2-D image of

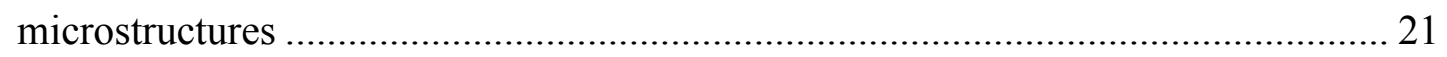

1.5.2 X-ray computed tomography (CT) for studying 3-D microstructure ......... 22

1.5.3 Transmission X-ray microscopy (TXM) to study 3-D microstructure ....... 23

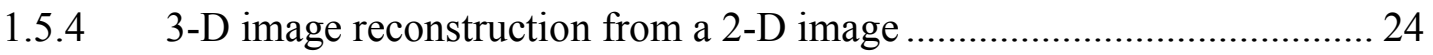

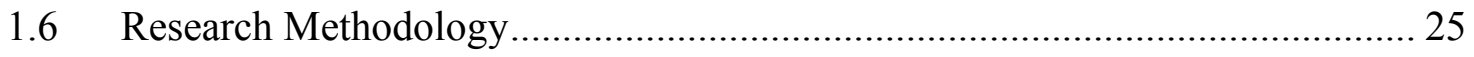

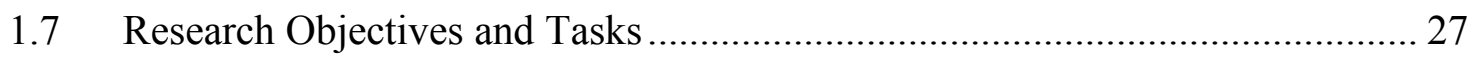

1.7.1 Task 1: Development and validation of computational fracture models ... 28 
1.7.2 Task 2: Pore pressure calculation and damage simulation on idealized samples

1.7.3 Task 3: Conducting controlled freeze-thaw test and microstructure image acquisition

1.7.4 Task 4: Numerical validation of crystallization pressure mechanism for frost-induced damage in cement-based samples .................................................. 32

CHAPTER 2 FRACTURE MODELS DEVELOPMENT FOR HETEROGENEOUS

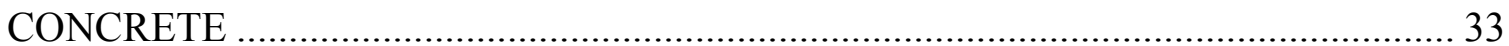

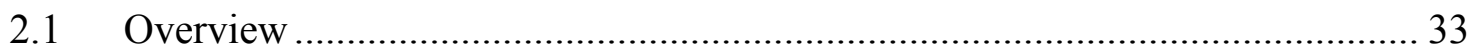

2.2 Extended Finite Element Method................................................................. 33

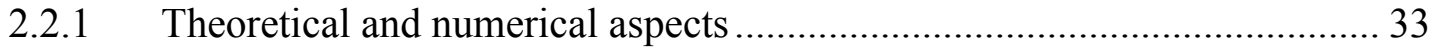

2.2.2 Fracture property prediction on uniform samples................................ 42

2.2.3 Fracture simulations on idealized cementitious sample.......................... 52

2.3 Bilinear Cohesive Zone Modeling Technique .............................................. 58

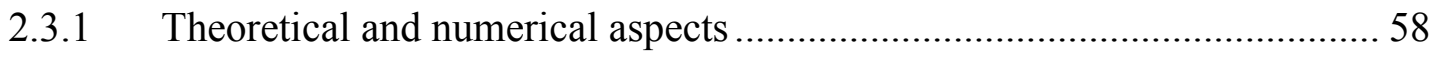

2.3.2 Fracture simulation on uniform or idealized samples............................. 71

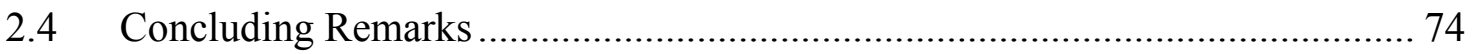

CHAPTER 3 EXPERIMENTAL TESTS ON CONCRETE AND CEMENT PASTE

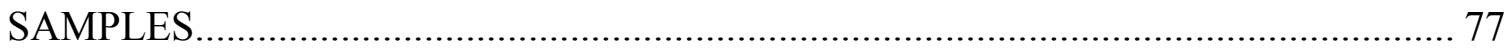

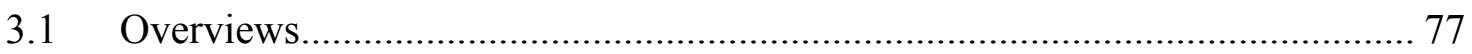

3.2 Concrete and Cement Paste Specimens Preparations ................................... 78

3.2.1 Concrete specimens preparations.................................................. 78

3.2.2 Cement paste specimens preparations ................................................... 81

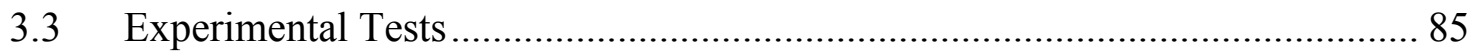

3.3.1 Single-edge-notched beam (SEB) bending test ................................... 85

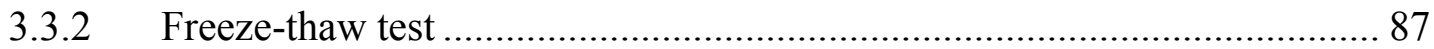




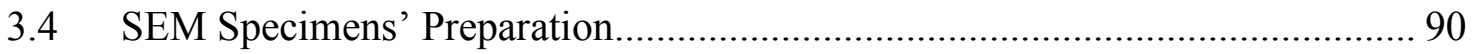

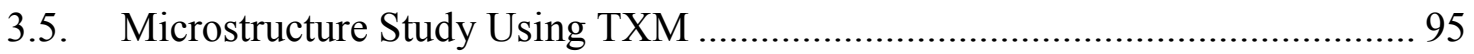

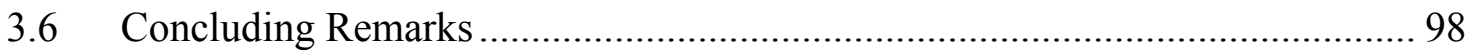

CHAPTER 4 IMAGE DATA ACQUISITION AND ANALYSIS ON CONCRETE AND

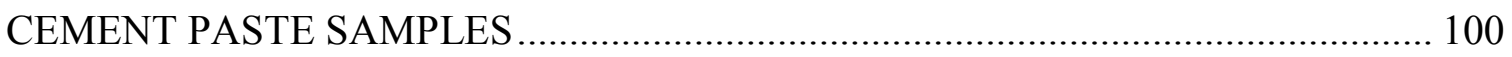

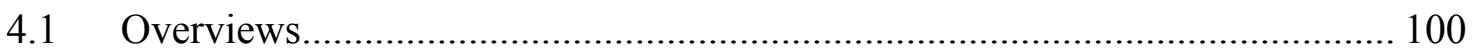

4.2 Image Analysis on Concrete Samples ............................................................. 101

4.3 Image Analysis on SEM Samples ............................................................... 104

4.3 TXM Sample Image Processing .................................................................. 116

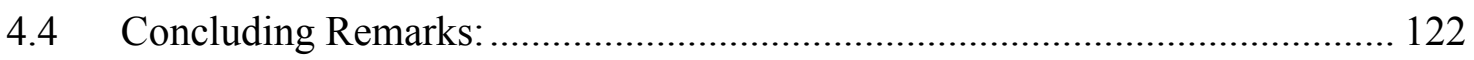

CHAPTER 5 INTERNAL FROST DAMAGE COMPUTATION WITH IDEALIZED PORES UNDER CRYSTALLIZATION PRESSURE............................................... 125

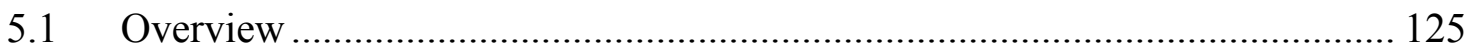

5.2 Internal Frost Damage Analysis within Idealized Pores of 2-D Samples under

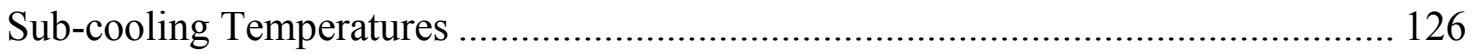

5.2.1 Scenario 1: Single pore system ................................................................ 127

5.2.2 Scenario 2: Multiple pore system ……………....................................... 131

5.3 3-D Fracture Simulation of Internal Frost Damage within Idealized Pore System 138

5.3.1 2-D damage simulation with one idealized pore in cement paste sample 138

5.3.1 3-D damage simulation with one idealized pore in cement paste sample 140

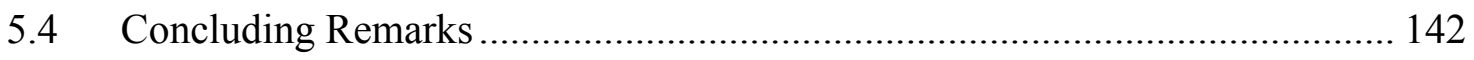

CHAPTER 6 VALIDATION OF MICROMECHANICAL ANALYSIS OF INTERNAL FROST DAMAGE AND FRACTURE TESTS WITH TESTED SAMPLES ................ 144

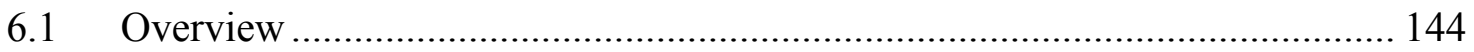

6.2 Computational Prediction and Validation with Concrete SEB Test ................. 145 
6.3 Computational prediction of fracture test and internal frost damage with 2-D

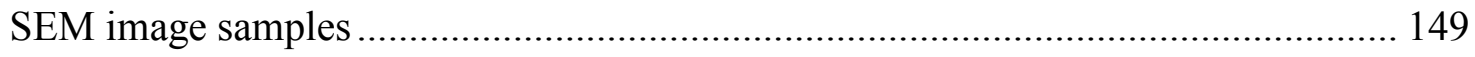

6.3.1 2-D SEM digital sample generation....................................................... 150

6.3.2 Computational simulation of 2-D SEM digital samples under CT test .... 151

6.3.3 Validation of internal frost damage 2-D simulation of SEM digital freezethawed damaged samples .................................................................................. 153

6.4 Computational Prediction of Internal Frost Damage with 3-D SEM Image

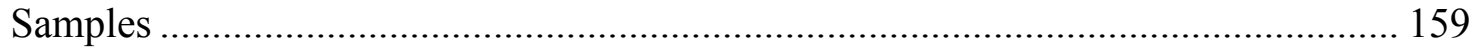

6.4.1 3-D SEM digital sample generation........................................................ 160

6.4.2 Computational simulation of internal frost damage with 3-D SEM digital

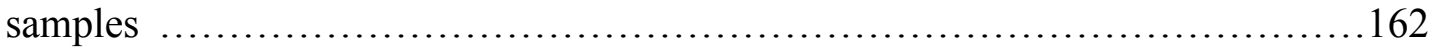

6.4 Computational Prediction of Internal Frost Damage with 3-D TXM Image

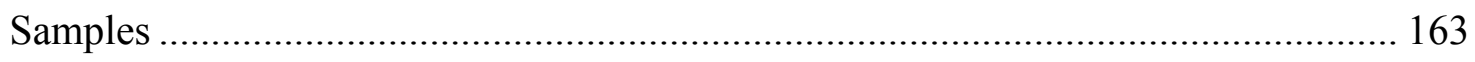

6.4.1 3-D TXM image processing and digital sample generation ..................... 164

6.4.4 Computational simulation and validation of internal frost damage with 3-D

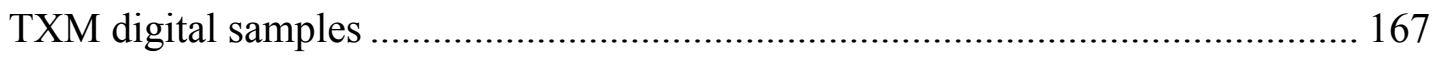

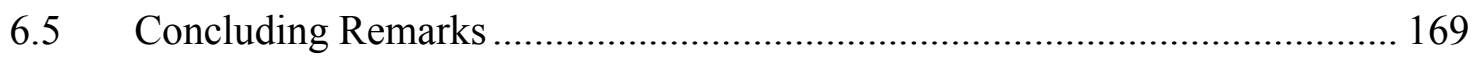

CHAPTER 7 CONCLUSIONS AND FUTURE WORKS........................................... 172

7.1 Summary of Research Investigations ........................................................... 172

7.2 Suggestions for Future Work ……………………................................. 175

7.2.1 In-situ TXM investigation of freeze-thaw damage of cement paste

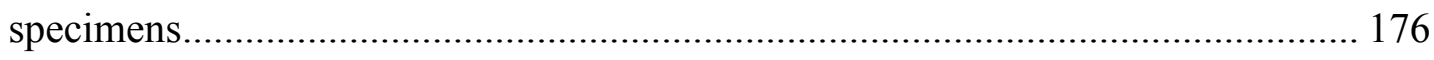

7.2.2 Employing XFEM technique for fracture simulation in a multi-pore system

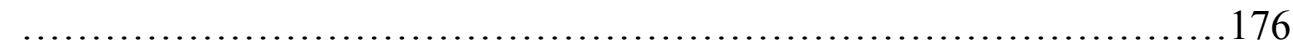

7.2.3 Generating predicted crack growth path from acquired image data ......... 177

7.2.4 In-situ micromechanical behavior investigation on cement paste sample 177

7.2.5 Statistical analysis on mixture design for concrete pavement ................. 178 
Appendix A: Developed MATLAB scheme to generate ABAQUS input file for implementing bilinear cohesive zone model (CZM).

Appendix B: Bilinear CZM user element subroutine (ABAQUS) for simulating fracture behavior in 3-D microstructure models .................................................. 211

Appendix C: Documents of permission to use copyrighted materials

Appendix C1: Copyright permission of journal titled: "Investigation of fracture behavior of heterogeneous infrastructure materials with extended-finite-element method and image anlaysis"

Appendix C2: Copyright permission of journal titled "Concrete fracture properties with compact tension test"

Appendix C3: Copyright permission of journal titled "Tailored extended finiteelement model for predicting crack propagation and fracture properties within idealized and digital cementitious material samples".

Appendix C4: Copyright permission of book titled "Concrete: microstructure, properties, and materials"

Appendix C5: Copyright permission of journal titled "A review of salt scaling: II. Mechanisms".

Appendix C6: Copyright permission of journal titled "Damage investigation of single-edge notched beam tests with normal strength concrete and ultra high performance concrete specimens using acoustic emission techniques". 


\section{LIST OF FIGURES}

Figure 1-1: Dimensional range for various types of voids and solid particles in a hydrate cement paste (Mehta and Monteiro 2006) - see permission in Appendix C4.

Figure 1-2: Schematic of capillary pore formation in cement paste during hydration. ...... 8

Figure 1-3: (a) Results that show that the introduction of air void reverse the volume change upon freezing (Valenza II and Scherer 2007) - see permission in Appendix C5.

(b) Compressive stresses (red arrows) are generated as the crystallization effect presence in the pore. (c) Schematic of ice in an air void and in a capillary pore. The negative pressure at the bulge surface $\mathrm{N}$ pulls the pore wall into compression.

Figure 1-4: Crystallization within a capillary pore with curvature varied which results different stress level induced on the pore wall or the crystal itself.

Figure 1-5: (a) Schematic of cohesive zone concept. (b) A closer look of the cohesive zone that made up of a crack tip and a cohesive zone tip.

Figure 1-6: Schematic flow diagram of proposed study on investigating the crystallization pore pressure effect within the concrete material.

Figure 1-7: Schematic flow of Task 1: Development and validation of computational fracture models

Figure 1-8: Schematic flow of Task 2: Computation and simulation of ice crystallization pressure within pore system.

Figure 1-9: Schematic flow chart of Task 3: Performing controlled freeze test on cement paste specimens and data acquisitions, which are acquired using SEM and TXM techniques.

Figure 1-10: Schematic flow chart of Task 4: Performing micro-damage model validations based on the freeze-test result of concrete specimens.

Figure 2-1: Schematic representations of Heaviside enrichment in $\Omega^{1}$ (circle) and crack tip enrichment in $\Omega^{2}$ (square) that defines a crack in the XFEM (Ng and Dai 2012) - see permission in Appendix C2 and C3.

Figure 2-2: Schematic of normal and tangent level set methods that represent the geometry of crack surface and crack front boundary ( $\mathrm{Ng}$ and Dai 2012) - see permission in Appendix C2 and C3 
Figure 2-3 Schematic representation of inclusion enrichments (red circles) (Ng and Dai 2012) - see permission in Appendix C3.

Figure 2-4: Numerical scheme for modeling the interaction between crack propagation and particles: for each crack increment $\Delta \alpha$, the crack propagation with the maximum energy release rate is determined by using a predefined search distance $d_{\mathrm{s}}$ and a number of kink angles $\beta$ ( $\mathrm{Ng}$ and Dai 2012) - see permission in Appendix C3......................... 42

Figure 2-5: Schematic representation of CT fracture test (Ng and Dai 2012) - see permission in Appendix C3, with standard dimension (Anderson 1995; Ng and Dai 2011).

Figure 2-6: XFEM simulation of CT testing with two crack growth length $a$, (a) the mesh plot with crack enrichments for $a=1.4 a_{0}$, (b) the contour plot of $\sigma_{y y}$ stress for $a=1.4 a_{0}$, (c) the mesh plot with crack enrichments for $a=3.0 a_{0}$, (d) the contour plot of $\sigma_{y y}$ stress for $a=3.0 a_{0}(\mathrm{Ng}$ and Dai 2012) - see permissions in Appendices C2 and C3.45

Figure 2-7: Validation simulation with an open-mode middle-notched SEB fracture test on a homogeneous sample: a) standard beam dimensions with boundary conditions, $b$ ) stress contour with the initial crack length, $a_{0}=20 \mathrm{~mm}$, and (c) $\sigma_{y y}$ contour plot with the crack length, $a=2.5 a_{0}(\mathrm{Ng}$ and Dai 2011) - see permission in Appendix C1

Figure 2-8: Singe-edge notched beam simulation with a uniform cement sample, (a) mixed-mode test geometry with offset notch, (b) element mesh with crack path and enrichment, (c) $\sigma_{y y}$ stress contour with crack path (Ng and Dai 2012) - see permission in Appendix C3.

Figure 2-9: Compact tension fracture simulation of idealized samples with circular particles on two different locations ( $e=3 d / 4$ and $d / 4)$ with respect to the crack line, (a) and (b) illustrate the mesh and $\sigma_{y y}$ stress contour with propagated crack line for particle A located at $e=3 d / 4$ respectively, (c) and (d) illustrate the mesh and $\sigma_{y y}$ stress contour with propagated crack line for particle A located at $e=d / 4$ respectively ( $\mathrm{Ng}$ and Dai 2012) - see permission in Appendix C3.

Figure 2-10: Compact tension fracture simulation of idealized samples consists of elliptical particles with shape factor of 1.5 at two orientations $\left(\beta=-20^{\circ}\right.$ and $\left.-45^{\circ}\right)$. (a) and (b) illustrate the mesh and $\sigma_{y y}$ stress contour with propagated crack line for particle oriented at $\beta=-20^{\circ}$, respectively. (c) and (d) illustrate the mesh and $\sigma_{y y}$ stress contour 
with propagated crack line for particle oriented at $\beta=-45^{\circ}$, respectively $(\mathrm{Ng}$ and Dai 2012) - see permission in Appendix C3

Figure 2-11: Compact tension fracture simulation of idealized samples that are consisted of elliptical particles oriented at $\alpha=-20^{\circ}$ with two shape factors ( $S F=2.67$ and 1.33), (a) and (b) illustrate the mesh and $\sigma_{y y}$ stress contour with propagated crack line for particles $S F=2.67$ respectively, (c) and (d) illustrate the mesh and $\sigma_{y y}$ stress contour with propagated crack line for particle $S F=1.33$ respectively ( $\mathrm{Ng}$ and Dai 2012) - see permission in Appendix C3.

Figure 2-12: Schematic plot of bilinear cohesive zone model which demonstrate a linear relation before reaching the peak load and decays to zero monotonically.

Figure 2-13: Schematic of a cohesive zone element with four nodes in the global (X-Y) coordinates for undeformed conditions and local coordinates for deformed case, with an orientation angle $\theta$.

Figure 2-14: Schematic 3-D cohesive zone element that is built up with 8 nodes (red spheres) in the global (X,Y, and Z) coordinates and local (n, s1, and s2) coordinates. With Side 1 consists of nodes 1, 2, 3, and 4, whereas Side 2 consists of nodes 5, 6, 7, and 8. . 67

Figure 2-15: A 2-D fracture simulation of CT test on a homogeneous cement paste sample by using bilinear CZM technique.

Figure 2-16: 3-D fracture simulation of CT test by using bilinear CZM technique. (a) A 3-D homogenous cement paste model. (b) A two phase cement paste model which shows the crack coalesces from one pore to another.

Figure 2-17: A 3-D fracture simulation of SEB test on a homogeneous cement paste sample by using bilinear CZM technique.

Figure 3-1: Compression test on concrete specimens to determine the failure stress. (a) The Baldwin Material Testing Equipment utilized for performing compression test. (b) A compression test conducted on a concrete specimen.

Figure 3-2: A cement paste freeze-thaw beam with 15.5 inches in length, 4 inches in width, and 3 inches in thickness.

Figure 3-3: Cylindrical cement paste specimen fabrications. (a) The mixed cement paste are filled into straws and let hardened for a day. (b) The hardened cement paste after removing straw molds. 
Figure 3-4: The setup for SEB bending test on concrete specimen using MTS machine with load fixture, fix support and roller support (Dai et al. 2012) - see permission in Appendix C6.

Figure 3-5: SEB bending test on a concrete specimen. (a) Boundary conditions and dimensions for the SEB bending test. (b) Developed crack growth path (red line) after the load reached $80 \%$ of the maximum applied load ( $\mathrm{Ng}$ and Dai 2012) - see permission in Appendix C3.

Figure 3-6: Freeze-thaw test on cement paste beam specimens. (a) The freeze-thaw chamber utilized for performing freeze-thaw test using ASTM C666 procedure A. (b) Six cement paste beams with different mixture designs (highlighted in red box) are to be

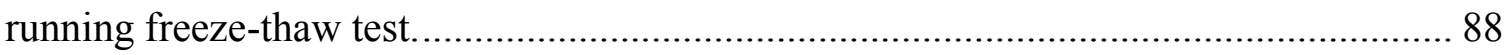

Figure 3-7: Cement paste beam specimens after freeze-thaw test with all specimens encounter complete failure, except for air entrainment specimens with w/c of 0.48 and 0.52 . Specimens in (a), (c), and (e) are without air entrainment with $\mathrm{w} / \mathrm{c}$ of $0.45,0.48$, and 0.52 , respectively. Specimens in (b), (d), and (f) were added with air entrainment with w/c of $0.45,0.48$, and 0.52 respectively.

Figure 3-8: The cylindrical cement paste samples on a Plexiglas that were prepared for microstructure study using SEM instrument.

Figure 3-9: Cement paste sample preparation for SEM specimen. (a) The cylindrical cement paste specimens were adhered on a Plexiglas with JB-Weld. (b) A container was made and filled with sands to cover the SEM specimen.

Figure 3-10: Vacuum impregnation on SEM specimen. (a) A vacuum chamber used for performing vacuum impregnation. (b) The SEM specimen with hardened fluorescent dyed epoxy.

Figure 3-11: Kerosene saw machine that was used to cut the SEM specimen into a thin slice, as illustrated in Figure 3-8.

Figure 3-12: The polisher lapping machines that were used to polish the SEM specimen. (a) The polisher lapping machine that used $9 \mu, 6 \mu$, and $3 \mu$ grits. (b) The polisher lapping machine that used $1 \mu$, and (c) The polisher lapping machine that used $0.25 \mu \ldots \ldots \ldots \ldots \ldots \ldots . . .95$

Figure 3-13: The TXM machine at the beam line of 32-ID that was used to study the cement paste microstructure. 
Figure 3-14: Specimen preparation before operating the TXM machine. (a) Cement paste that was adhered on pin by using clay, followed by (b) welding with a small amount of gold for alignment purpose.

Figure 4-1: Image processing on a SEB concrete sample. (a) The concrete beam sample was scanned using high resolution scanner and the image was reduced to $30 \%$ of its original size. (b) The scanned image was converted to binary image and threshold was applied to differentiate between cement mastic and aggregate phases ( Ng and Dai 2012) see permission in Appendix C3.

Figure 4-2: Digital sample generation on the concrete beam from Figure 4-1 for the XFEM and CZM models. (a) Imaging processing and ellipse fitting techniques applied in the fracture domain, and (b) fitted ellipses in the finite element mesh plot (Ng and Dai 2012) - see permission in Appendix C3. (c) A bilinear CZM model of the concrete beam was developed that follows the actual aggregates' geometries and sizes.

Figure 4-3: A digital SEM image of an undamaged cement paste sample with w/c=0.47 was processed to generate fracture model. (a) A section of 100x100 pixels with pore structures was selected, and (b) the image was processed for noise reduction.

Figure 4-4: The constructed FE model of CT test with standard dimensions and boundary conditions.

Figure 4-5: A SEM image of a freeze-thawed cement paste specimen with a $\mathrm{w} / \mathrm{c}=0.47$. (a) Cracks developed in the cement paste microstructure due to crystallization pressure, and an interested area (as shown with red box) was selected for CZM simulation and validation. (b) The selected image section is cropped, resized, filtered, and rotated to develop digital sample for CZM simulation. (c) The processed image was edited to patch the crack with matrix phase for fracture model simulation.

Figure 4-6: The constructed FE model based on the digital SEM image on damaged cement paste sample.

Figure 4-7: Image processing on SEM image of an undamaged cement paste sample with $\mathrm{w} / \mathrm{c}=0.47$. (a) An SEM image with dimension of $300 \times 300$ pixels. (b) Image processing on SEM image after threshold of 40 in magnitude was applied.

Figure 4-8: The 3-D image reconstruction technique was applied on the SEM image of an undamaged cement paste sample with $w / c=0.47$ : (a) A random 3-D image slice was generated with a porosity of $6.35 \%$. (b) A 3-D image slice after executing sintering algorithm to remove noises in the image. 
Figure 4-9: 3-D image reconstruction generation on SEM image of an undamaged cement paste sample with $\mathrm{w} / \mathrm{c}=0.47$ : (a) A 3-D reconstructed image after stacking 300 image slices. (b) A 3-D view of pore structure for the reconstructed 3-D image.

Figure 4-10: Schematic highlights the steps on 3-D image reconstruction from an SEM undamaged cement paste sample with $\mathrm{w} / \mathrm{c}=0.47$

Figure 4-11: The digital image of a freeze-thawed cement paste microstructure acquired from the TXM (a) before and (b) after image processing............................................ 117

Figure 4-12: A 3-D image was constructed from the 2-D TXM digital image slices using the ImageJ 3-D Viewer technique. 118

Figure 4-13: The cement paste microstructure TXM image after filtered using median filtering technique. 118

Figure 4-14: A cross section of $300 \times 300$ pixels was selected for cropping through Specify option in ImageJ (http://rsb.info.nih.gov/ij/).

Figure 4-15: The result of re-stacked on cropped cement paste microstructure TXM image with image size of $300 \times 300 \times 200$ pixels.

Figure 4-16: A threshold of 62 in magnitude was applied as it demonstrates apparent pore structure in the ImageJ (http://rsb.info.nih.gov/ij/).

Figure 4-17: A 3D view of $300 \times 300 \times 200$ pixels of cement paste microstructure TXM image that shows only the pore structures.

Figure 5-1: Schematic illustration of crystallization pressure effect within a pore system in the cement paste (a) before reaching the threshold temperature $\left(-7.69^{\circ} \mathrm{C}\right)$, with a pressure $P_{A}=7.52 \mathrm{MPa}$. (b) As the ice at free ends grows into capillary pore after reaching the threshold temperature with pressure $P_{B}=4.62 \mathrm{MPa}$ based on GibbsThomson formula. 128

Figure 5-2: XFEM simulation of pore system as the threshold temperature $\left(-4.85^{\circ} \mathrm{C}\right)$ had reached and ice grew into capillary pore. (a) The principal stress $\sigma_{1}$ on a pore with radius of $50 \mathrm{~nm}$ and $P_{A}=4.15 \mathrm{MPa}$. (b) The principal stress $\sigma_{1}$ contour generated on the $15 \mathrm{~nm}$ channel with $P_{B}=2.9 \mathrm{MPa}$. 131

Figure 5-3: Schematic illustration of multiple pore system with capillary pores. (a) At $T=$ $-2{ }^{\circ} \mathrm{C}$, ice was formed in spherical pore 1 and bulged into the cylindrical pore channel 1 , (b) at $T=-3.58^{\circ} \mathrm{C}$, ice was formed in Pore 2 and bulged into the cylindrical pore channel 1 with hemispherical free ends, (c) at $T=-7.51^{\circ} \mathrm{C}$, ice invaded through cylindrical pore 
channel 1 and bulged into cylindrical pore channel 2 with hemi-sphere ends, and (d) slightly below threshold temperature $T=-7.51^{\circ} \mathrm{C}$, the ice is penetrating into the cylindrical pore channel 2 with one free hemispherical end.

Figure 5-4: XFEM simulation $\sigma_{1}$ contour plot for multiple pores scenario with crack generated since the hoop stress generated was greater than the tensile strength of cement paste. Simulation results at temperature slightly below $-7.51^{\circ} \mathrm{C}$ in: (a) Pore 1 with $P_{A}=$ $7.34 \mathrm{MPa}$ at point A and (b) Pore 2 with $P_{B}=6.19 \mathrm{MPa}$ at Point B.

Figure 5-5: XFEM fracture simulations $\sigma_{1}$ contour plot on cylindrical capillary pore channels 1 and 2 at the temperature slightly below $-7.51{ }^{\circ} \mathrm{C}$ in: (c) Channel 1 with $P_{C}=$ $6.86 \mathrm{MPa}$ at point $\mathrm{C}$, and (d) Channel 2 with $P_{D}=4.51 \mathrm{MPa}$ at point D. The views for both channels 1 and 2 are in the plane perpendicular to this page.

Figure 5-6: Schematic illustration of node $\left(\mathrm{N}_{1}, \mathrm{~N}_{2}, \mathrm{~N}_{3}\right.$, and $\left.\mathrm{N}_{4}\right)$ and surface (S1, S2, S3, and S4) numbering of a four-node bilinear square element......

Figure 5-7: A 2-D bilinear CZM fracture simulation in ABAQUS on an idealized pore with $10 \mu \mathrm{m}$ in radius in cement paste as a result of crystallization pore pressure, $\mathrm{P}_{\mathrm{a}}$. The enlarged view showed the crack opening at Point $A$ as a result of crystallization pore pressure.

Figure 5-8: Schematic illustration of node and surface numbering of a cubic 8-node reduced integration element.

Figure 5-9: A 3-D bilinear CZM fracture simulation in ABAQUS on an idealized pore with $10 \mu \mathrm{m}$ in radius in cement paste as a result of crystallization pressure.

Figure 6-1: XFEM simulation of singe-edge middle-notched beam test on a concrete specimen: (a) image of the tested specimen with indicated propagated crack line shown in red, (b) mesh plot with predicted crack path in the digital sample, (c) $\sigma_{y y}$ stress contour with crack propagation (Ng and Dai 2012) - see permission in Appendix C3............. 146

Figure 6-2: Fracture simulation of the concrete SEB test by using bilinear CZM technique in ABAQUS. (a) The SEB model in ABAQUS with deformation and fracture developed after a unit displacement is applied on the model. (b) A section of detail view of the SEB model that displays the crack growth pattern developed mostly along the cementaggregate interface.

Figure 6-3: CZM fracture simulation of CT test with SEM digital cement paste sample. (a) The von Mises stress contour plot of the FE model at the initial stage of CT test. (b) 
At the final stage of the FE model, the CT test experiences complete failure with concentrated stress around the loading and fixed points.

Figure 6-4: FE fracture simulation result with applied crystallization pressure: (a) The constructed FE model with fracture domain defined as highlighted in dotted line box. (b) An enlarged view of fracture zone above Pore A. (c) and (d) show the crack opening along the cement and clicker particle boundaries. 155

Figure 6-5: Bilinear CZM simulation result in ABAQUS on cement paste due to applied crystallization pressure: (a) The mesh constructed in ABAQUS with CZM boundary defined in yellow dotted line. (b) and (c) show the enlarged view crack opening in the highlighted area along the predefined crack path as a result of crystallization pressure on Pore A.

Figure 6-6: Bilinear CZM simulation result in ABAQUS on top half of the cement paste model: (a) The mesh constructed in ABAQUS with CZM boundary defined in the highlighted area (yellow dotted boundary). (b) An enlarged view that shows the crack opening along the hydrated-unhydrated cement interface. (c) A predefined crack growth path developed in the model with crack opening and (d) an enlarged view that shows the crack opening along the hydrated-unhydrated cement interface as a result of crystallization pressure from Pore B... 158

Figure 6-7: Validation of bilinear 2-D CZM fracture simulation result to the actual fracture sample. The highlighted area A and B show the simulated crack agreed well with the actual crack developed in the damaged cement paste sample.

Figure 6-8: Image slices that contain pores were obtained from the reconstructed 3-D image of the cement paste sample with a w/c of 0.47 and a porosity of $6.35 \%$.

Figure 6-9: 3-D fracture simulation of internal frost damage analysis on reconstructed 3$\mathrm{D}$ image for the cement paste sample with a w/c of 0.47 and a porosity of $6.35 \%$. The highlighted area display the crack developed as a result crystallization pressure effect. 163

Figure 6-10: One of the TXM image slices that showed a crack pattern in the highlighted box in ImageJ (http://rsb.info.nih.gov/ij/).

Figure 6-11: The six selected TXM image slices that displayed crack path: (a) The actual image samples before noise removal and (b) the filtered images with noises removed. 166

Figure 6-12: Validation of the fracture simulation by comparing the damaged cement paste sample with a w/c $=0.47$ : (a) The constructed FE model based on the TXM image slices of the cement paste sample with predefined crack path (bilinear CZM elements) as indicated in dotted yellow line. (b) One of the slice images with actual crack path as 
highlighted in yellow dotted line. (c), (d) and (e) The enlarged view of the crack opening in the FE model as a result of the crystallization pore pressure. The damages on the cement paste sample were validated by the computational simulation results............... 168 


\section{LIST OF TABLES}

Table 2-1: Comparison of calculated and theoretical stress intensity factor $K_{I}$ from XFEM simulation and fracture mechanics analysis (Ng and Dai 2012)..................................... 46

Table 2-2: Comparison of $K_{I}$ of a SEB fracture test between analytical results from XFEM simulation and theoretical values from fracture mechanics analysis (Ng and Dai

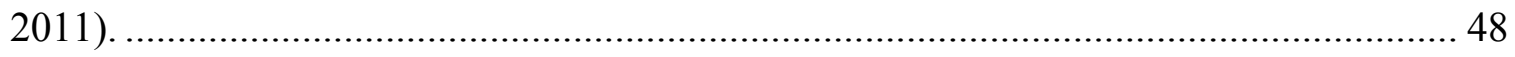

Table 3-1: List of ASTM Standards (ASTM 2009) ………………………………….... 78

Table 3-2: Aggregate sizes and gradation in the mixture design ..................................... 79

Table 3-3: Specific weight and weight ratio of mixture contents ................................... 80

Table 3-4: Compressive strengths of concrete specimens for different curing periods.... 80

Table 3-5: Mixture contents for preparing cement paste freeze-thaw beams ................... 83

Table 5-1: Freezing temperatures in the pore system for scenario 2............................ 132

Table 6-1: Material properties and cohesive fracture parameters for different phases in concrete (Mehta and Monteiro 2006; Roesler et al. 2007) ........................................... 147

Table 6-2: Material properties and cohesive fracture parameters for different phases in

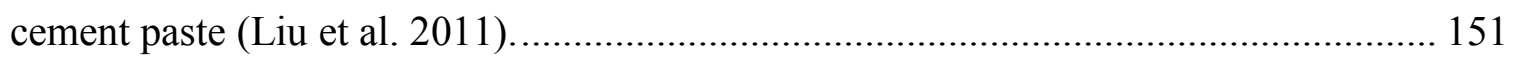




\section{ACKNOWLEDGEMENTS}

I would like to give my special thanks to my advisor, Dr. Qingli Dai, who gave me the opportunity to work on this challenging project and has brought me a continuous motivation, research enthusiasm, and strong supports. I also would like to thank and appreciate her continued guidance, patience and friendly attitude provided me the inspiration to get familiar with civil engineering materials and complete my Doctoral study. I have the deepest respect and sincere admiration to her.

I wish to extend my sincere thanks to Dr. Jacob Hiller, Dr. Zhanping You, and Dr. Ibrahim Miskioglu for serving as committee members and their help. I would like to acknowledge the National Science Foundation for their financial support for this project. I am also indebted to Mr. Jerry Anzalone, Mr. Dale Bentz from National Institute of Standards Technology (NIST), Mr. Matthew Pais from Dassult Systèmes Simulia Corp., Mr. Steve Wang from Advanced Photon Source (APS) in Argonne National Laboratory, and Dr. Karl Peterson from University of Toronto, for their helps and precious suggestions throughout my research study. My special thanks are extended to Mr. Rob Fritz, Mr. Benjamin Roskoskey, Mr. Michael La'Court, Mr. Eric Kreiger, and Mr. Michael Yokie for helping in the experimental works on concrete specimens and mixture designs.

Finally, I wish to appreciate the unflinching support, endless love and encouragement of my family and my wife. One special thankful note will go with my housemate, Yong Meng, Sua who has encouraged and supported me to throughout my research study. 


\title{
ABSTRACT \\ INTEGRATION OF COMPUTATIONAL MODELS AND EXPERIMENTAL CHARACTERIZATION TO STUDY INTERNAL FROST DAMAGE IN CEMENTITIOUS MATERIALS
}

\author{
By Kenny Ng
}

The objective of this doctoral research is to investigate the internal frost damage due to crystallization pore pressure in porous cement-based materials by developing computational and experimental characterization tools. As an essential component of the U.S. infrastructure system, the durability of concrete has significant impact on maintenance costs. In cold climates, freeze-thaw damage is a major issue affecting the durability of concrete. The deleterious effects of the freeze-thaw cycle depend on the microscale characteristics of concrete such as the pore sizes and the pore distribution, as well as the environmental conditions. Recent theories attribute internal frost damage of concrete is caused by crystallization pore pressure in the cold environment. The pore structures have significant impact on freeze-thaw durability of cement/concrete samples. The scanning electron microscope (SEM) and transmission X-ray microscopy (TXM) techniques were applied to characterize freeze-thaw damage within pore structure. In the microscale pore system, the crystallization pressures at sub-cooling temperatures were calculated using interface energy balance with thermodynamic analysis. The multi-phase Extended Finite Element Modeling (XFEM) and bilinear Cohesive Zone Modeling (CZM) were developed to simulate the internal frost damage of heterogeneous cementbased material samples. The fracture simulation with these two techniques were validated by comparing the predicted fracture behavior with the captured damage from compact tension (CT) and single-edge notched beam (SEB) bending tests. The study applied the developed computational tools to simulate the internal frost damage caused by ice crystallization with the two dimensional (2-D) SEM and three dimensional (3-D) reconstructed SEM and TXM digital samples. The pore pressure calculated from thermodynamic analysis was input for model simulation. The 2-D and 3-D bilinear CZM predicted the crack initiation and propagation within cement paste microstructure. The favorably predicted crack paths in concrete/cement samples indicate the developed bilinear CZM techniques have the ability to capture crack nucleation and propagation in cement-based material samples with multiphase and associated interface. By comparing the computational prediction with the actual damaged samples, it also indicates that the ice crystallization pressure is the main mechanism for the internal frost damage in cementitious materials. 



\section{CHAPTER 1 INTRODUCTION}

\subsection{Background}

Concrete is largely produced as a major construction material in the contemporary world with about 11 billion metric tons every year (Mehta and Monteiro 2006; Scherer and Valenza 2005; Valenza II and Scherer 2007). According to the Portland Cement Association, more than 340 million yards of concrete are placed each year in America (Sullivan 2006). Concrete has been playing an important role in United States transportation, but more specifically, the Interstate Highway System in the past 50 years. Approximately $60 \%$ of the interstate highway system is made of concrete pavement (http://www.cement.org/pavements/pv_cp_highways.asp 2012). Concrete is still widely used for pavement construction compared to asphalt because of following characteristics:

(1) High strength: With less deformation (no rutting) and higher stiffness, this enables concrete to support heavy traffic loads in today's world.

(2) Low cost: With more than sufficient resources, concrete is inexpensive and cost $25 \%$ to $50 \%$ less compared to asphalt (http://www.pa.pavement.com/why.htm 2010). Besides, concrete is durable (maximum 40 years) with minimal maintenance required that indirectly cuts the cost for rebuilding or repairing.

(3) Others: Concrete is recyclable, easy on repairment, and safer for drivers. These are among the advantages that have been considered in pavement design.

The complex behavior of concrete is determined by its highly heterogeneous and complex microstructures. So what is concrete composed of? According to ASTM C 12507, concrete is defined as "a composite material that consists essentially of a binding 
medium within which are embedded particles or fragments of aggregate. In hydraulic cement concrete, the binder is formed from a mixture of hydraulic cement and water" (ASTM 2009; Mehta and Monteiro 2006). Aggregates are classified as sand, gravel, crushed stone or other demolition products (e.g. recycling of concrete and brick). When aggregates are mixed with cement, new composites such as concrete and mortar are produced with increased strength. Mortar is a mixture of sand, cement, and water without gravel or coarse aggregates. Cement is a finely crushed and dry material that reacts with water through hydration to develop a binding property. The term hydraulic in the definition of concrete means the hydration products that are stable in a wet environment. The most widely used hydraulic product for making concrete is Portland cement, which consists of reactive calcium silicates that create adhesive characteristics after mixed with water during the hydration. Because of this adhesive characteristic, the product of cement and water is called binder. Depending on the design constraints or environmental factors, admixtures (elements other than aggregate, cement, and water) can be introduced in the concrete design to enhance the concrete properties. For instance, air entrainments are added to introduce air bubbles in the concrete in order to create space for ice to expand upon freezing to reduce the freeze-thaw damage (Mamlouk and Zaniewski 2006; Mehta and Monteiro 2006).

Even though concrete is a durable product, the traffic loading variables and environmental factors could cause a mass deterioration during its service life. In the cold climate region with sub-cooling temperatures, frost damage has been identified as one of major causes that affect concrete's durability. The factors contributing to the frostinduced damage include the internal frost damage, salt scaling damage, and freeze-thaw 
damage (Scherer and Valenza 2005; Valenza II and Scherer 2007). Both salt scaling damage and freeze-thaw damage are categorized as superficial damages. The salt scaling damage occurs when a pool of saline solution freezes on the concrete surface, while the freeze-thaw damage occurs when a critically saturated concrete experiences distresses after several freeze-thaw cycles. On the other hand, the internal frost damage has been suggested as the main cause that leads to the frost-induced durability issues of the concrete pavement (Scherer and Valenza 2005; Valenza II and Scherer 2007). The internal frost damage is illustrated as the damage caused by the ice that induces crystallization pore pressure in the saturated capillary pores (Scherer and Valenza 2005; Valenza II and Scherer 2007). The pressure generation is not only because of the transition from water to ice that leads to a volume expansion of approximately $9 \%$, but also the pressure level difference between the pores. The later scenario can be explained based on thermodynamic principles, which will be discussed in the next section.

Until now, the cause of the internal frost damage is still inconclusive due to limited critical references in this field. That is, the crystallization pore pressure effect is still unclear and there is no experimental evidence or computational simulation that shows the crystallization pore pressure effect in the concrete microstructure. Hence, further study is needed to explore the crystallization pore pressure effect on concrete microstructure. In this dissertation study, the mechanism of the internal frost damage has been investigated through performing microstructure-based modeling with applied thermodynamic principles and laboratory tests. It is also essential to understand the characteristics and behavior of concrete from the microstructural aspects. Therefore, both 
numerical and experimental analyses need be carried out simultaneously to investigate the internal frost damage in concrete.

Scanning electron microscope (SEM) and X-ray computed tomography (CT) have been implemented to capture the concrete microstructure. The SEM technique has been applied to acquire two-dimensional (2-D) images for studying engineering properties and microstructure of cement-based materials (Kyritsis et al. 2009) and for reconstructing periodic 3-D microstructure models (Garboczi et al. 1999; Liang et al. 1998). The concrete microstructure study is not limited to 2-D images only, 3-D images also can be acquired through the X-ray $\mathrm{CT}$ technique. The X-ray $\mathrm{CT}$ technique has been applied recently to investigate the microstructure and to predict the material properties of pavement materials (Dai 2011; Dai et al. 2005; You et al. 2009) by capturing the threedimensional (3-D) microstructure with imaging processing. The X-ray CT technique has been employed for studying transport property (Bentz et al. 2000a; Bentz and Martys 1994) and permeability (Sumanasooriya et al. 2010) of cement-based materials.

Likewise, fracture modeling on pavement materials has also been developed extensively in the last decade to investigate and to predict the fracture behaviors within the materials by using a finite element (FE) analysis technique, such as extended finite element modeling (XFEM) (Dai and Ng 2010; Dai et al. 2011; Ng and Dai 2011; 2012) and cohesive zone modeling (CZM) (Kim et al. 2008; Kim et al. 2007; Paulino et al. 2004; Song et al. 2006a; b). With these available techniques, the investigation of the frost induced damage process in the cement-based material can be performed based on the thermodynamic principles developed by Scherer (Scherer and Valenza 2005; Scherer 1999). This dissertation study will enhance the fundamental understanding of the internal 
frost-damage in concrete, and will also provide guidance in concrete pavement design and maintenance.

\subsection{Literature Review on Concrete Microstructure}

It is essential to study the characteristics of concrete microstructure as the internal frost damage is suggested to be caused by the crystallization pore pressure in the microstructure. Concrete is a porous heterogeneous material. It is a mixture of cement, water, and aggregates. Some admixtures will be added depending on the design constraints. Without aggregates added for strengthening purposes, the mixture of just cement and water will produce a hydration product called cement paste. The beauty of this hydration product formation is mainly because of the chemical composition in clinker compounds: $\mathrm{C}_{3} \mathrm{~S}, \mathrm{C}_{2} \mathrm{~S}, \mathrm{C}_{3} \mathrm{~A}$, and $\mathrm{C}_{4} \mathrm{AF}$. These chemical composition terms are abbreviated and will be used in this dissertation, in which $\mathrm{C}=\mathrm{CaO}, \mathrm{S}=\mathrm{SiO}_{2}, \mathrm{~A}=$ Al2O3, $\mathrm{F}=\mathrm{Fe}_{2} \mathrm{O}_{3}, \overline{\mathrm{S}}=\mathrm{SO}_{3}$, and $\mathrm{H}=\mathrm{H}_{2} \mathrm{O}$. The phases that can be found in the cement paste are as follows (Mehta and Monteiro 2006):

(1) Calcium silicate hydrate or C-S-H

(2) Calcium hydroxide or $\mathrm{C}-\mathrm{H}$

(3) Calcium sulfoaluminates hydrates

(4) Unhydrated clinker grains

(5) Pore spaces.

It is important to understand these phases as the properties of these phases govern the macroscopic properties of concrete. Figure 1-1 (Mehta and Monteiro 2006) illustrates 
the dimensional range of various types of voids and solid particles in a hydrated cement paste.

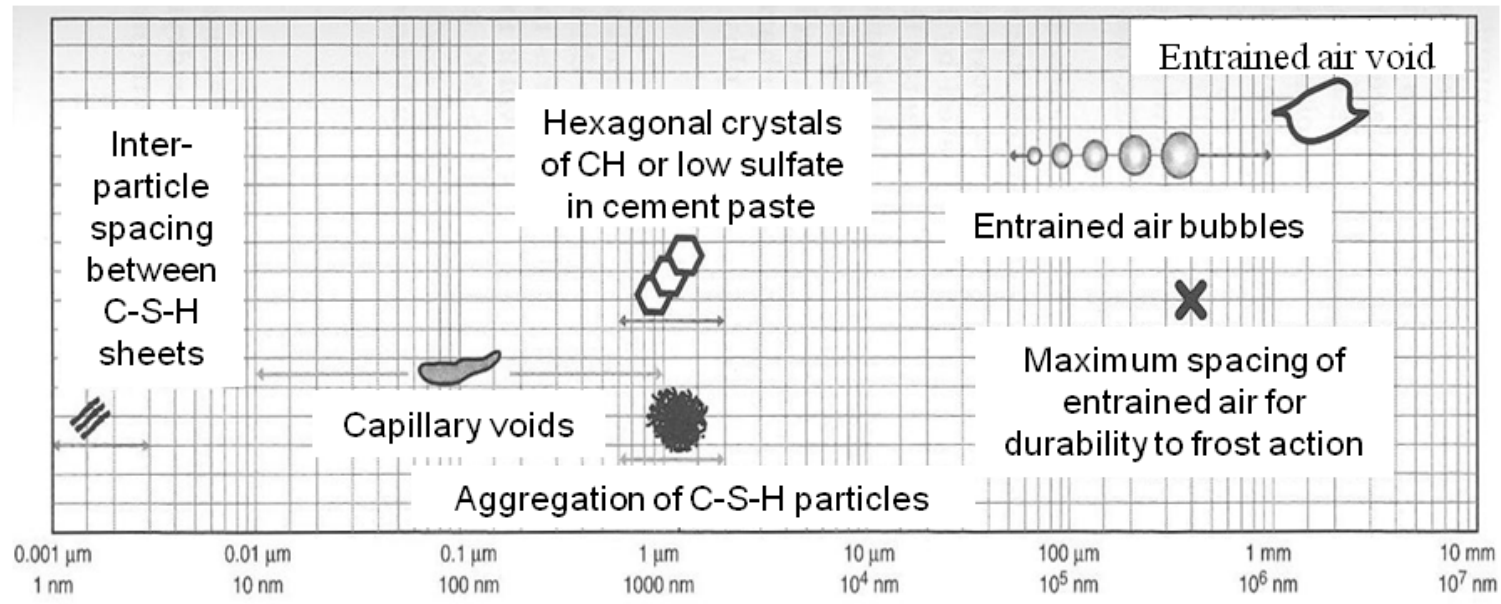

Figure 1-1: Dimensional range for various types of voids and solid particles in a hydrate cement paste (Mehta and Monteiro 2006) - see permission in Appendix C4.

$\mathrm{C}-\mathrm{S}-\mathrm{H}$ is the most important phase that determines the cement paste properties due to its 50 to 60 percent of volume occupancy of solids in a completely hydrated cement paste (Brandt 1995; Mehta and Monteiro 2006). The strength of the material is mainly contributed by the van der Waal's forces. Some works have been done to determine the elastic modulus of this phase. Mondal (Mondal 2008) has identified that the $\mathrm{C}-\mathrm{S}-\mathrm{H}$ phase has an elastic modulus in the range of $10 \mathrm{GPa}$ to $30 \mathrm{GPa}$ from a nanoindentation hardness test. C-H, which is in the form of a large crystal, composes $20 \%$ to $25 \%$ of the solid volume in hydrated cement paste. Due to lower surface area, the contribution of calcium hydroxide to the strength of cement paste is limited. Calcium sulfoaluminates hydrates or ettringite constitutes 15 to 20 percent of the solid volume in hydrated cement paste, and thus, is an unimportant phase to determine the strength of 
material. The remaining solid phase in the hydrated cement paste is the unhydrated clinker grains which are formed depending to the degree of hydration. The elastic modulus is determined to be approximately $100 \mathrm{GPa}$ (Mondal 2008) from the nanoindentation hardness test.

There are three types of voids within the hydrated cement paste: (1) interlayer space in C-S-H, (2) capillary voids, and (3) air voids (Mehta and Monteiro 2006). The interlayer space in C-S-H or so called the gel pores, occupy $40 \%$ to $50 \%$ of total pore volume of cement paste (Brandt 1995). It is the smallest void that ranges from 0.5 to 2.5 $\mathrm{nm}$. The space is too small to be filled with hydration products and thus, does not have significant effect on the strength and permeability of the hydrated cement paste. However, it is believed that the gel pores may contribute to shrinkage and creep properties of the cement paste.

Next, capillary pores may range from $10 \mathrm{~nm}$ to $5 \mu \mathrm{m}$. The capillary pores with sizes larger than $50 \mathrm{~nm}$ are known as macro-pores, which determine the strength and impermeability characteristics in concrete. Whereas, the capillary pores smaller than 50 $\mathrm{nm}$ are known as micro-pores, which are known to cause drying shrinkage and creep (Mehta and Monteiro 2006). Finally, the air voids (size range from 50 to $200 \mu \mathrm{m}$ ) are generally spherical and are formed as a result of a small amount of air that gets trapped in the cement paste during the concrete mixing (Mamlouk and Zaniewski 2006; Mehta and Monteiro 2006; Pigeon and Pleau 1995). 


\subsubsection{Capillary pores}

Figure 1-2 illustrates the development of capillary pores as a result of hydration in the cement paste. In the early stage of hydration, water will react actively with cement grains or clinkers to generate new hydration products, or solid phases as mentioned in the previous section (Figure 1-2 (a) and (b)). As the hydration progresses, the spaces that originally filled with water will be occupied by the growing dense cement gels which contact each other. The remaining spaces (Figure 1-2(c)) are called capillary pores (Mehta and Monteiro 2006; Pigeon and Pleau 1995). The unhydrated cement grains will remain embedded within the cement gel and will continue to involve in hydration but not active, depending on the amount of water available for hydration.

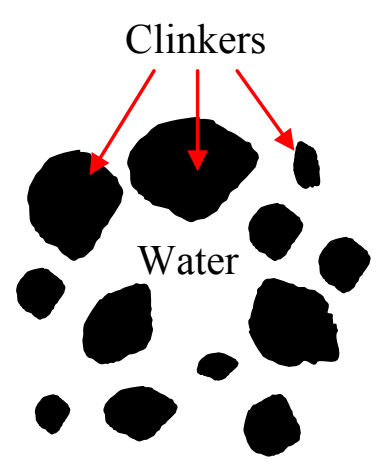

(a)

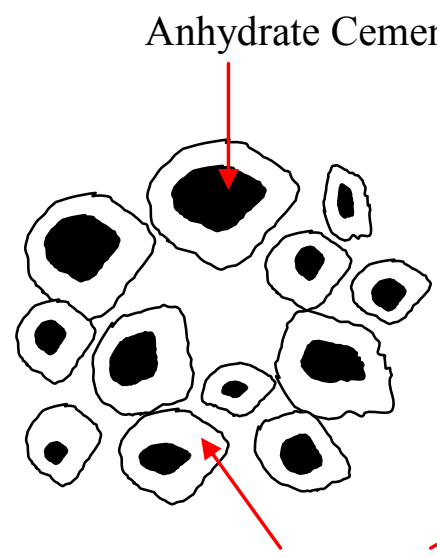

Dense Cement Gel

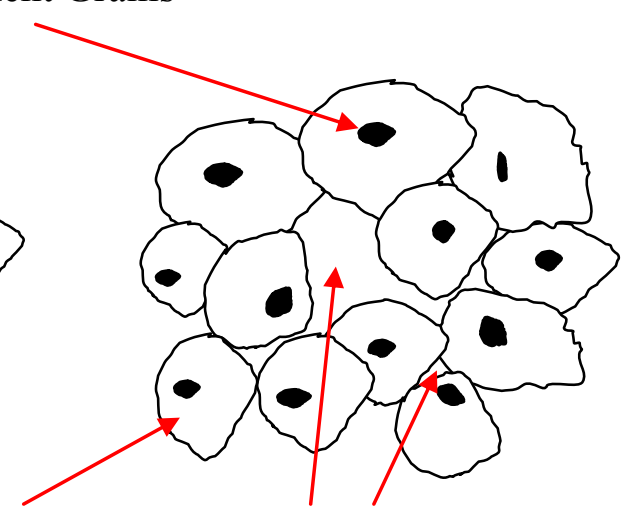

Capillary Pores

(c)

Figure 1-2: Schematic of capillary pore formation in cement paste during hydration.

The capillary pores may remain fully or partially saturated, depending on the atmospheric humidity and the involvement of cement grains in hydration. Meanwhile, the water to cement ratio $(\mathrm{w} / \mathrm{c})$ influences the amount and sizes of capillary pores in cement 
paste. For a high w/c (> 0.5), the capillary pore sizes and quantities are higher due to an excess of water for hydration, and vice versa for low w/c. It has been observed that a w/c of $0.35-0.40$ will provide sufficient amount of water to ensure a full hydration in the cement paste (Brandt 1995).

\subsection{Literature Review of Internal Frost Damage on Concrete}

Not all water contain in the pores will turn into ice in the freezing temperature. Study has shown that the freezing temperature decreases as the pore size decreases (Pigeon and Pleau 1995). Thus, the ice will form in the larger pores first. In fact, the lowest winter temperature in the settled zones in the world seldom reaches below $-40{ }^{\circ} \mathrm{C}$. Besides, based on Litvan's theory (Litvan 1975), the mobility limitation of water that were tightly held by the C-S-H in cement paste prevents the water to rearrage and to form ice at the normal freezing temperature. Generally, the more tightly water is held (such as in smaller pores), the lower the freezing point. Hence, the water will freeze only in some capillary pores and air voids but not in the interlayer space in C-S-H. Therefore, the study of internal frost damage to concrete has been focusing on capillary pores and air voids.

The studies of internal frost damage have been carried out for over a century but still, no conclusive answers have been reached on the actual process that causes the damage of hydrated concrete materials. In the early studies, Powers (Mehta and Monteiro 2006; Pigeon and Pleau 1995; Powers 1949; Powers 1975; Scherer and Valenza 2005; Valenza II and Scherer 2007) proposed that hydraulic pressure is the main factor that creates stresses and causes damage to concrete. According to Powers, when the ice forms in the saturated pores, the ice expands in volume and occupies most of the spaces in the 
pores. As a result, unfrozen water is pushed away and thus, the hydraulic pressure is built up. Based on this theory, Powers suggested a well distributed closely spaced air voids in concrete will create a reservoir for storing unfrozen water that is pushed away by the ice in the pores and therefore, prevent the hydraulic pressure to be built up.

Powers' theory was then found to be unimportant on frost damage to concrete after Powers and Helmuth (Powers and Helmuth 1953) revised the theory and claimed that the air void can only provides more room for ice to nucleate rather than acting as a reservoir. Besides, the ice in the void will draw in water at the intersection pore (location $\mathrm{N}$ in Figure 1-3(c)) from surrounding small pores and thus, exerts compressive stresses on concrete. This causes the solid matrix to compress to occupy empty spaces in the pore channels and therefore leads to shrinkage in volume, as shown in Figure 1-3 (a) (Valenza II and Scherer 2007). When no air entrainment is added, the volume of concrete expands, as shown in Figure 1-3 (b). This scenario occurs as the ice in the small pores induces crystallization pore pressure. The crystallization pore pressure is generated as a result of van der Waals repulsive forces between solid phases (Scherer 1999), which are the ice and mineral in the pore walls. The repulsive forces are sufficient to surpass concrete tensile strength and thus, lead to damages and generate cracks. The theory of crystallization pore pressure was strengthened later by Scherer's thermodynamic principles (Pigeon and Pleau 1995; Scherer and Valenza 2005; Valenza II and Scherer 2007), which shows the significance of the crystallization pore pressure to cause damage to concrete. 


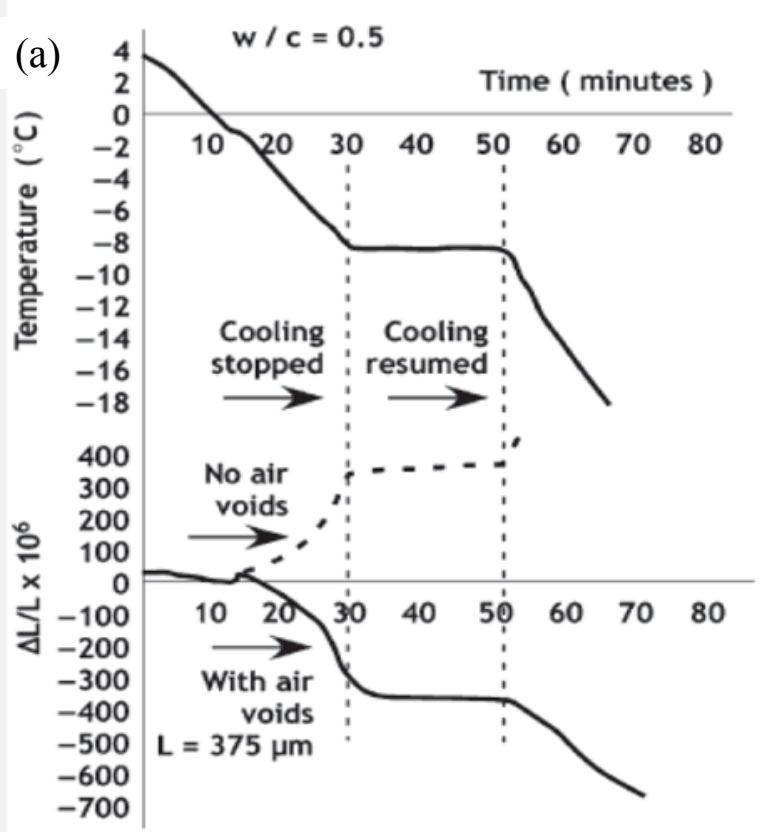

(b)

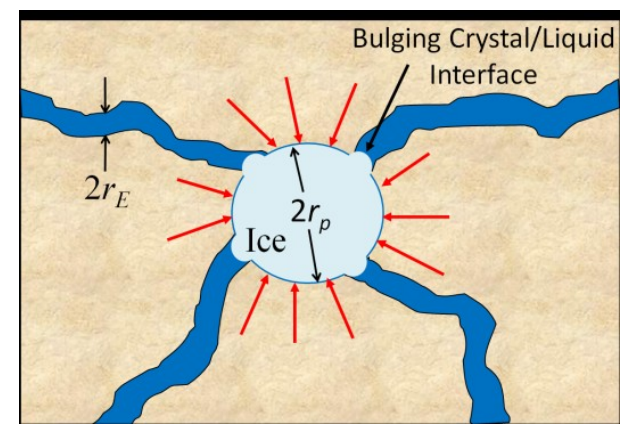

(c)

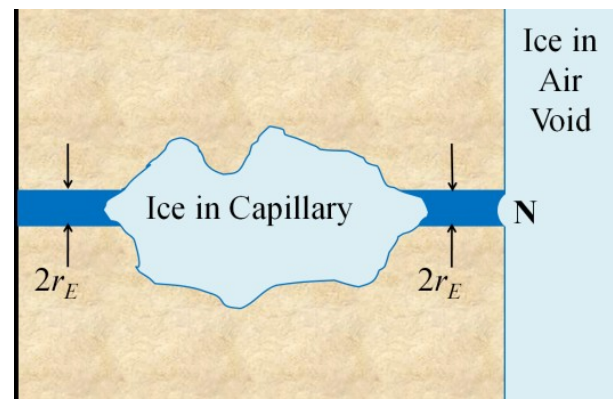

Figure 1-3: (a) Results that show that the introduction of air void reverse the volume change upon freezing (Valenza II and Scherer 2007) - see permission in Appendix C5. (b) Compressive stresses (red arrows) are generated as the crystallization effect presence in the pore. (c) Schematic of ice in an air void and in a capillary pore. The negative pressure at the bulge surface $\mathrm{N}$ pulls the pore wall into compression.

Before analyzing the crystallization pore pressure, it is important to understand the relationship between pore size and freezing/melting temperature. The relationship can be explained based on thermodynamic principles (Kaufmann 2000; Scherer and Valenza 2005; Scherer 1999). The melting temperature $\left(T_{m}\right)$ for ice in a spherical pore which has a radius of $r_{p}$ can be explained by the Gibbs-Thomson equation (Scherer and Valenza 2005; Valenza II and Scherer 2005) as below:

$$
T_{m}\left(\kappa_{C L}\right)=T_{m}(0)-\frac{\gamma_{C L} \kappa_{C L}}{\Delta S_{f v}}
$$


where $T_{m}\left(\kappa_{C L}\right)$ is the threshold temperature for forming crystals in the capillary pores, $\gamma_{C L}$ is the crystal/liquid interfacial energy which is approximately $0.04 \mathrm{~J} / \mathrm{m}^{2}$ (Brun et al. 1977), $\kappa_{C L}=2 /\left(r_{p}-\delta_{W}\right)$ is the curvature of the crystal/liquid interface related to spherical pore radius $\left(r_{p}\right)$ and the thickness of unfrozen water layer $\left(\delta_{W}\right)$, which is approximately $0.9 \mathrm{~nm}$ for water (Brun et al. 1977), and $\Delta S_{f v}$ is the molar entropy of fusion which is approximately $1.2 \mathrm{~J} /\left(\mathrm{cm}^{3} \cdot \mathrm{K}\right.$ ) (Brun et al. 1977) or equivalent to $\left(S_{L}-S_{C}\right) / v_{C}$, where $v$ is the molar volume, while the subscripts $L$ and $C$ stands for liquid and crystal, respectively.

In the last decade, Scherer and Valenza has extended the thermodynamic principles to predict the crystallization pore pressure by including the relationship between the stress and interfacial energies (Scherer and Valenza 2005). After some manipulations, the equilibrium state for an ice crystal in pure water is given as,

$$
v_{c}\left(P_{A}+\gamma_{C L} \kappa_{C L}\right)=\left(v_{L}-v_{c}\right)\left(P_{L}-P_{e}\right)+\left(S_{L}-S_{C}\right)\left(T_{m}-T\right)
$$

where $P_{A}$ is the additional pressure applied to prevent the growth of crystal, while $v_{L}$ and $v_{C}$ are the molar volume of liquid and crystal, respectively. From Laplace's equation, which relates the pressure in the liquid to the curvature of the liquid/vapor interface, $\left(P_{L}-P_{e}\right)=\gamma_{L V} \kappa_{L V}$, in which $\gamma_{L V}$ is the surface tension of the liquid/water interface. Hence, Equation (1-2) is rearranged and is given as:

$$
v_{c}\left(P_{A}+\gamma_{C L} \kappa_{C L}\right)=\left(v_{L}-v_{c}\right) \gamma_{L V} \kappa_{L V}+\left(S_{L}-S_{C}\right)\left(T_{m}-T\right)
$$


Figure 1-4 demonstrates the highest pressure that is generated in an irregular shape of pore or ice with small entries. The relatively flat surface (small curvature) leads the Point $\mathrm{P}$ to have the highest pore pressure induced to ice crystal. While the negative curvature at point $\mathrm{N}$ leads to very high pressure on the wall. The very small curvature at the channel $\mathrm{C}$ has the pressure of $P_{A} \approx \gamma_{C L} \kappa_{C L}^{E}$. In general, the equation for computing the pore pressure is as follow:

$$
P_{A}=\gamma_{C L}\left(\kappa^{E}-\kappa^{S}\right)
$$

where, $P_{A}$ is the pore pressure, $\kappa^{E}$ is the curvature of the capillary pore channel and $\kappa^{S}$ is the curvature of the pore surface.

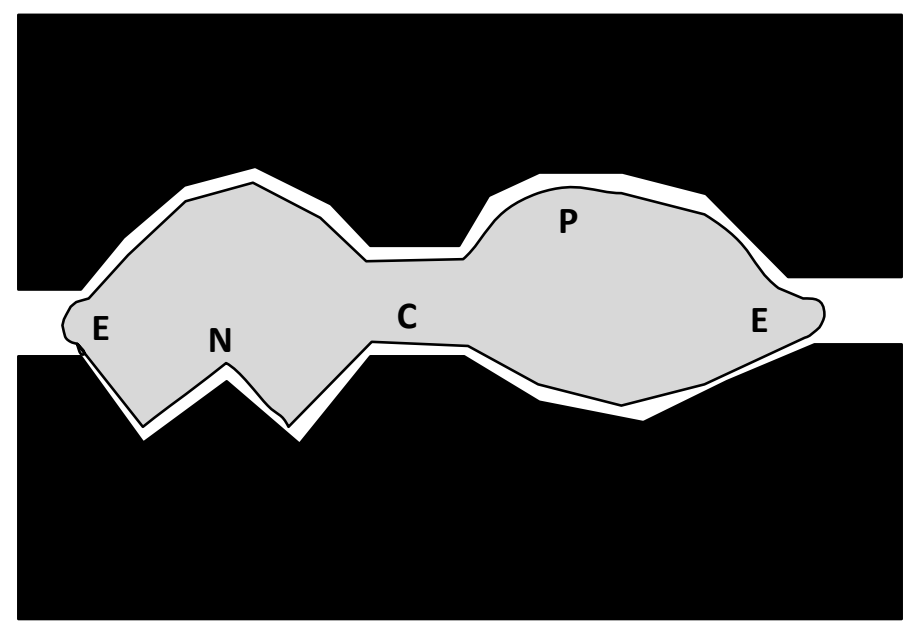

Figure 1-4: Crystallization within a capillary pore with curvature varied which results different stress level induced on the pore wall or the crystal itself.

Similarly, the internal frost damage in the scenario in Figure 1-3(b) can be explained based on the formulated thermodynamic principles. As illustrated in Figure 
1-3(b), the pore is filled with ice with a radius of $r_{p}$ and an unfrozen water layer of $\delta_{W}$ at the melting temperature, $T_{m}\left(\kappa_{C L}\right)$. When the temperature reaches below $T_{m}\left(\kappa_{C L}\right)$, the ice will bulge and penetrate into the surrounding entrance of the capillary pore channels with radius $r_{E}\left(<r_{P}\right)$ until the curvature satisfies Equation (1-1) (Scherer and Valenza 2005).In order to reach to an equilibrium state, a confining pressure (red arrows in Figure 1-3(b)) will be generated by the pore wall on the crystal's surface. As a result, a tensile hoop stress will be generated in the pore wall. According to Equation (1-4), the confining pressure is relative to the curvature difference between the surface of the crystal bulging into the capillary pore channel and the surface of the pore wall. Hence, the pressure will be maximized when the pore wall has a radius larger than the surface of bulging crystal at the intersecting pore. This confining pressure will initiate micro-cracks and then deteriorate the concrete as a result of the internal freezing (Valenza II and Scherer 2005).

One may argue the existence of an unfrozen water layer can resist the damage due to the ice expansion. This is not true because the van der Waals forces that generate repulsive force between the ice and the minerals in the pore walls are significantly large enough to surpass the tensile strength of concrete (Scherer and Valenza 2005; Scherer 1999). The ice can generate a pressure of approximately 1.2 MPa for a degree of undercooling temperature reduction. Thus, only a few degrees of under-cooling temperature is required to induce stresses to overcome the concrete's tensile strength, which is approximately $2 \mathrm{MPa}$ (Scherer and Valenza 2005). 


\subsection{Literature Review on Micro-damage Modeling}

\subsubsection{Micro-mechanical modeling of damage behavior}

Rather than spending great deal of time on lab specimen preparation and testing to investigate the material and fracture properties of the concrete, the cost-effective numerical computational work could be carried out. For over a hundred years, the micromechanical models have been studied for non-interacting and interacting particles. Among the pioneers in this area are (Voigt 1889), (Einstein 1906), and (Reuss 1929). Until recently, the FE modeling and discrete element (DE) modeling have been widely applied not only in macro scale but also in micromechanical analysis. For instance, a FE model of microstructure-based asphalt mixture was developed by Dai and You to predict the creep and complex moduli of asphalt mixture sample (Dai and You 2005; Dai and You 2007b). The complex constitutive behavior of stone-based materials were also studied through FE modeling, such as nonlinear visco-elastic behavior (Buttlar et al. 2006; Dai and You 2007a; Dai et al. 2006), visco-plastic behavior (Dai 2010), and anisotropic behavior (Guddati et al. 2002). On the other hand, DE modeling were developed to predict compressive dynamic moduli of asphalt mixtures subjected to uniaxial compression tests (You and Buttlar 2006) and complex modulus of asphaltaggreage under internal pressure (You and Buttlar 2005).

It is imperative that stone-based materials can endure various loading and temperature conditions for the field applications. For example, stone-based materials can be fractured or damaged under traffic loadings varying from quasi-static to dynamic impact. Recent studies on the failure and fracture behavior of stone-based materials include the cohesive fracture finite element modeling (Song et al. 2006a; b), the discrete 
element fracture simulations (Kim et al. 2009; Kim et al. 2008) and micro-frame finite element network (Dai and Sadd 2004; Dai et al. 2005; Sadd et al. 2004a).

The frost-induced damage has been investigated with computational analysis and experiments in recent decades. Zhou and Mihashi (Zhou and Mihashi 2008) studied strain behavior of concrete during freezing process through micromechanics model. Their theoretical prediction on thermal strain by considering phase transition, pore pressure and mass transfer within pore system was compared with experimental measurement on mortar samples. One mesoscale constitutive model were developed to simulate the frost damage with a two-dimensional rigid body spring model by Ueda (Ueda et al. 2009). The experimental work has been carried to monitor heat release, mechanical deformation and damage during freeze-thaw cycles with calorimetric, expansion and acoustic methods (Kaufmann 2004). This measurement helps to provide the physical understanding of the ice formation in small pores. Recently, the effects of air voids on internal freezing were investigated through the combination of calorimetric measurements and dilatometry. It was observed that thermal expansion and crystallization pore pressure occurred in airentrained or non-air entrained mortar samples during freeze-thaw cycles (Sun and Scherer 2010a). Sun and Scherer also investigated the ice grows in dendritic manner in cement paste (Sun and Scherer 2010b). Liu et al. (Liu et al. 2011) simulated internal damage of saturated cement paste with computer generated concrete microstructure and one 3-D lattice fracture model. Furthermore, the stress-strain relationship of frostdamaged concrete specimens subjected to fatigue loading was investigated (Hasan et al. 2008). 
The results had shown an agreement between the actual test results and the micromechanical model prediction. Further development of micromechanical modeling has been carried out and will be discussed in the following sections. For this work, the thermodynamic approach will be applied in the numerical analysis to model the internal frost damage in concrete.

\subsubsection{Extended Finite Element Method (XFEM) for fracture behavior}

The FE method has been widely applied for the material and structural analysis, not only in stress and deformation but also fracture behavior of a material (Soares et al. 2003). Since the traditional FE method is formulated with continuous media, additional remeshing efforts are needed to accurately predict the irregular crack propagation (Abdelaziz and Hamouine 2008). Over the past several decades, many techniques have been developed to perfrom the crack growth simulation including the enriched finite element method (Fan et al. 2004), boundary element method (Cruse 1988) and mesh-free Galerkin method (Belytschko et al. 1994). To avoid the mesh refinement in every step of crack propagation, a discontinuous enrichment technique was recently incorporated into the FE formulation to predict the crack path based on the partition of unity methods (Melenk and Babuska 1996).

The extended finite element method (XFEM), firstly proposed by Belytschko and Black (Belytschko and Black 1999), has been recently developed to reduce or eliminate the remeshing efforts associated with traditional FE modeling. Crack growth with minimal remeshing was firstly developed with an enriched finite element approximation (Belytschko and Black 1999). This formulation was improved to eliminate the remeshing 
efforts for simulating the crack propagation (Moës et al. 1999). This approach employed the elastic asymptotic fields for crack near-tip discontinuity and displacement jump function across the crack surfaces. The Level Set Method (LSM) (Osher and Sethian 1988) was introduced to track the crack geometry including the crack path and tip locations in the XFEM (Stolarska et al. 2001). With the assistance of LSM, the XFEM was applied to determine the necessary stress and displacement fields in order to generate the crack path. Besides the crack problems, the inclusions and voids have also been incorporated into the complex material system for the fracture study (Sukumar et al. 2001). With these developed techniques, the XFEM was used to analyze the fracture properties and the crack propagation of idealistic cement-based materials with typical laboratory tests (Ng and Dai 2011; 2012).

\subsubsection{Cohesive zone modeling (CZM) for fracture behavior}

The microstructure of idealized cement-based materials includes the cement paste, particles and interfacial boundaries. To understand the fracture phenomena in these materials, the continuum-based nonlocal theories were used with the complete stressstrain diagram in the hardening and softening regions plus the internal length scale (Bazant and Pijaudier-Cabot 1988; Sadd et al. 2004b; Sadd et al. 2004c; Wriggers and Moftah 2006). The continuum-based theories cannot describe the phase transformation phenomenon that the pre-critical micro-cracks reach critical macro-crack stage (van Mier 2008). In order to simulate the crack initiation and propagation, the cohesive zone modeling (CZM) has been developed over the past decades (Ortiz and Pandolfi 1999) and has been tailored over time to suit the application needs and constraints. 
The CZM techniques employ the relations between crack opening displacement and surface traction (Spring 2011). In the early 1933, the idea of cohesive traction relation was applied by Prandtl (Prandtl 1933) to predict the debonded zone length between two slender beams. The CZM, however, was firstly introduced in the early 60 s by Barenblatt (Barenblatt 1959), to study interaction forces between atoms near a crack tip in perfectly brittle materials. This method was found to be favorable to analyze fracture behavior of composite materials as the singularity issue can be avoided. During the 90s, Xu and Needleman had advanced the CZM technique with a potential-based CZM by introducing cohesive elements into a finite element mesh by using an exponential cohesive law (Shet and Chandra 2002; Song et al. 2006a; Xu and Needleman 1994). In this approach, the traction increases initially as the displacement between cohesive elements increases, followed by decaying monotonically after reaching a maximum displacement. To reduce the artificial compliance because of the elasticity of the intrinsic cohesive law, Espinosa and Zavattieri adopted bilinear CZMs by introducing a changeable initial slope in the cohesive law (Espinosa and Zavattieri 2003). (Kim et al. 2008; Kim et al. 2007; Song et al. 2005) had used the CZM approach to study the properties of asphalt mixtures, such as predicting nonlinearity of viscoelastic damage and fracture failure as well as fatigue and healing characterization. And Park et al. extended the model to simulate the fiber-reinforced concrete with the aggregate bridging zone and the fiber bridging zone (Park et al. 2010), while Li et al. conducted fracture studies on an adhesively-bonded polymer-matrix composite by using mixed-mode CZM.

On the other hand, Song implemented tailored CZM, such as a bilinear CZM and intrinsic CZM, to solve some limitations on traditional CZM to model fracture of asphalt 
concrete (Song et al. 2006a; b). For instance, bilinear CZM had been adopted to alleviate the compliance issue because of the elasticity of the intrinsic cohesive law by introducing a changable initial slope in the cohesive law (Song et al. 2006a; b). Roesler et al. has applied the bilinear CZM developed by Song to conduct concrete fracture prediction (Roesler et al. 2007). The solving of compliance issue and the successful fracture modeling on concrete by using bilinear CZM technique had encouraged this doctoral study to apply the numerical modeling technique for performing fracture modeling on cement-based material.

As illustrated in Figure 1-5 with an opening mode (pure Mode I), the CZM can simulate damage in the fracture area before reaching to a crack tip based on the nonlinear constitutive laws. The constitutive laws can be explained by the displacement jump, $\delta$ and traction, $t$ along interfaces to simulate fracture behavior such as crack nucleation, initiation, and propagation (Song et al. 2006b). At the material crack tip, the traction is zero while at the cohesive zone tip, the traction reaches maximum, as shown in Figure $1-5(b)$. The cohesive zone is made up of the region between the material crack tip and the cohesive zone tip where complex fracture behavior occurs, such as inelasticity. The cohesive surfaces are bound by cohesive traction, which is governed by the displacement jump across crack faces. With an applied force, the displacement jump and traction increase and reach to maximum, and ultimately decays to zero monotonically. The cohesive parameters affecting the separation-material response include material strength, critical displacement, and cohesive fracture energy. 
(a)

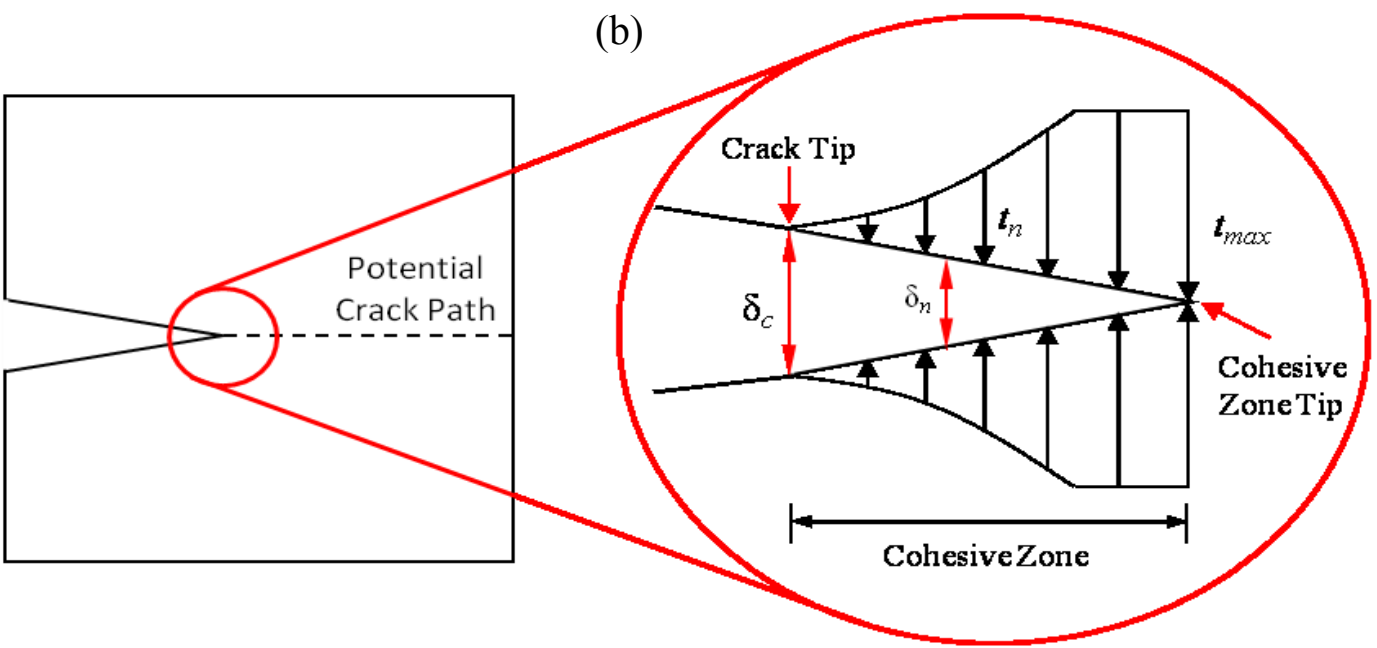

Figure 1-5: (a) Schematic of cohesive zone concept. (b) A closer look of the cohesive zone that made up of a crack tip and a cohesive zone tip.

\subsection{Literature Review on Image Acquisition and Processing Techniques}

With the recent development of advanced technologies, it is possible to view and analyze the microstructure within a material in micro- and nano- scale. There are two techniques that can be applied to capture the image of microstructure of a material; they are 2-D surface scanning and 3-D X-ray scanning.

\subsubsection{Scanning electron microscope (SEM) on capturing 2-D image of microstructures}

Surface scanning electron microscope (SEM) is the typical technique for 2-D surface scanning to obtain 2-D image of microstructures. For SEM imaging, the tested specimens need be sawn, ground, polished, and cleaned to obtain a surface of interest (Jacobsen et al. 1995). The surface of interest from the processed sample can be then observed and studied through the microscope. The observed SEM image is the result of 
secondary electrons, backscattered electrons, and X-rays after a high energy beam of electrons interacted on the surface of interest (Marusin 1995).

In most of the concrete work that had been performed, the microstructures, chemical compositions, and pore size distributions within concrete cement paste have been observed by using SEM (Jacobsen et al. 1995; Mehta and Monteiro 2006; Mouret et al. 1999; Terzic and Pavlovic 2010). Due to the process of surface removal and polishing, the structure might change significantly and new cracks might be introduced (Jacobsen et al. 1995). In addition, the lowest scale that SEM can reach is micrometer level. The Material Science Department in Michigan Technological University has the SEM facility that is specifically designed to study stoned-based material and capable to study material microstructures at the highest resolution of $5 \mu \mathrm{m}$.

\subsubsection{X-ray computed tomography (CT) for studying 3-D microstructure}

The X-ray computed tomography (CT) is a novel technology that was firstly applied in geological application (Ketcham and Carlson 2001). Within last two decades, the application has been extended in other industries on investigating the microstructures in other opaque materials in various scales (Bentz et al. 2000b; Ketcham and Carlson 2001). Comparing to the SEM technique, the X-ray CT is a non-destructive technique as no sawing and polishing are required for capturing the digital information of internal structure from the zone of interest (Dai 2011). The captured digital images will form a 3D image based on the density of objects.

The sample preparation and operation specifications of the X-ray CT scanning process have been based on the University of Texas High-Resolution X-ray CT Facililty 
(http://www.ctlab.geo.utexas.edu/eng/index.php). In the sample preparation, it is ideal to have cylindrical geometry of an object by coring or packing in a cylindrical container. The cylindrical container can be either X-ray-transparent filler or having a similar material density of the tested object. Upon obtaining the 3-D image data, 2-D image slices can be obtained to observe the cross section of interested area. Dai (Dai 2011) and Al-Omari (Al-Omari and Masad 2004) are among the examples who had performed Xray CT scanning on asphalt material, and applied the X-ray data to perform numerical modeling. Dai had used the X-ray CT imaging technique to perform FE simulation to predict dynamic modulus and phase angle on asphalt material, while Al-Omari (Al-Omari and Masad 2004) used the X-ray CT images to simulate fluid flow within asphalt material. On the other hand, Bentz and Martys applied the X-ray microtomography to capture the 3-D image of concrete to study permeability and conductivity (Bentz and Martys 1994). And Bentz et. al. also utilized the X-ray microtomography to capture the microstructure and to study the transport properties of concrete (Bentz et al. 2000a).

\subsubsection{Transmission X-ray microscopy (TXM) to study 3-D microstructure}

Transmission X-ray Microscopy (TXM) is available in Advanced Photon Source (http://www.aps.anl.gov/Users/Prospective/) in Argonne National Lab. The TXM technique is capable to perform fast image acquisition $(50 \mathrm{~ms}$ time resolution and phase contrast enhancement) with $30 \mathrm{~nm}$ resolutions that help revealing detailed nano-structure and dynamic process or reaction within specimen (Chen et al. 2010; Hsu et al. 2010). A nano-tomography image of specimen can be captured too. The only limitation of this sophisticated instrument is that it only can observe a specimen with thin layer. The 
technique offers several advantages that will further assist the investigation of the crystallization process within capillary pores in nano scale. The technique offers several advantages that will further assist the investigation of the crystallization process within capillary pores in nano scale.

\subsubsection{3-D image reconstruction from a 2-D image}

The acquired 2-D image can be constructed to a 3-D image to further analyze the fracture behavior in the cement based microstructure by maintaining the properties of the parent 2-D image. Dale Bentz has developed programs, which is available at $\mathrm{ftp} / /$ ftp.nist.gov/pub/bfrl/bentz/permsolver/, to randomly reconstruct 3-D images of cement based microstructures from 2-D digital images, such as SEM images (Bentz and Martys 1994; Garboczi et al. 1999; Sumanasooriya and Neithalath 2009). The developed 3-D microstructure models were applied in permeability prediction (Sumanasooriya et al. 2010), drying shrinkage analysis (Bentz et al. 1998), and transport properties determination (Quenard et al. 1998). Only pore and solid phases were constructed in a model for a porous media, with the pore phase was assigned as the main phase to be studied in the model. There are three techniques for developing the 3-D microstructure: (1) correlation functions measurement, (2) 3-D image reconstruction, and (3) sintering algorithm.

First, the correlation functions measurement is executed to generate information of the pores in the microstructure, which include the area fraction, characteristic sizes, and the specific surface area of the pores (Sumanasooriya et al. 2010). This is done through the two-point correlation (TPC) functions which measure the pore structure 
statistically (Bentz and Martys 1994; Berryman 1985). The generated data are then applied in the 3-D image reconstruction algorithm to generate 2-D image slices followed by stacking the 2-D image slices to form a 3-D image. The constructed 3-D image has hydraulic radii smaller than the parent 2-D image and consists of noises. The hydraulic radii are the ratio of the cross-section area to the perimeter of pores, or the fraction of the total volume to the total surface area of pores. The sintering algorithm (Bentz et al. 1991; Pimienta et al. 1992) is executed to generate the 3-D microstructure with hydraulic radii closer to the original 2-D image and to eliminate the noise. The algorithm employs the relationship between the pore curvature and pixel counting. This is done by moving the pixels from the solid phase with the highest curvature to the pore phase with the lowest curvature. The cycles repeat until the desired hydraulic radius is reached.

\subsection{Research Methodology}

There are challenges on carrying out this doctoral study as a result of inconclusive answer about the internal frost damage in the concrete. Nevertheless, the available resources and technologies allow the tasks for the doctoral study to be performed. In the engineering world, just a theory is not sufficient to prove whether it is valid or not. Hence, the experimental and numerical works have to be carried out to validate the theories. In this doctoral study, both experimental and numerical works were performed to investigate the internal frost damage with generated crystallization pore pressure that causes damages in concrete based on the thermodynamic principle. The flow diagram of this doctoral study is as follows: 


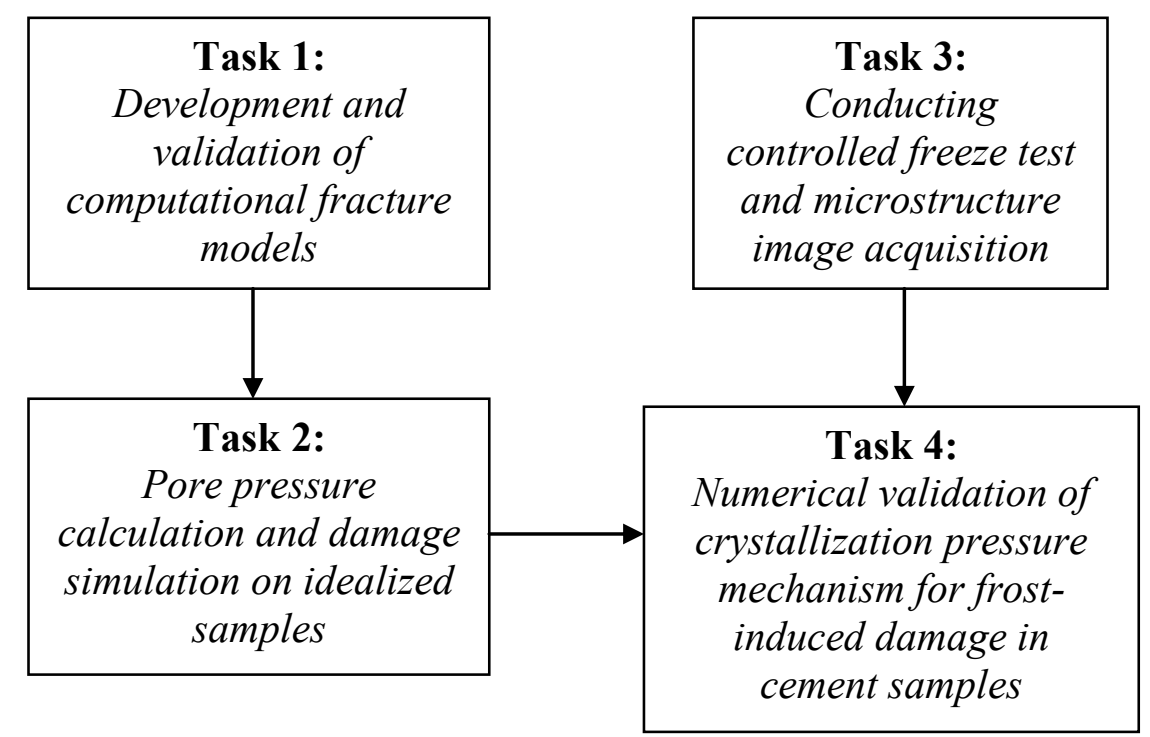

Figure 1-6: Schematic flow diagram of proposed study on investigating the crystallization pore pressure effect within the concrete material.

The followings are a brief description of each task:

\section{Task 1: Development and validation of computational fracture models}

Develop the computational fracture models by using XFEM and bilinear CZM techniques to simulate crack propagation within concrete and cement paste media under mechanical loadings. The model simulation on crack propagation is based on image samples that will be validated with the fracture or crack patterns developed in the tested specimens.

\section{Task 2: Pore pressure calculation and damage simulation on idealized samples}

Develop a MATLAB program to compute the ice crystallization pore pressure within a pore system under sub-cooling temperatures. The computed pressure magnitudes 
will be employed to simulate the frost-induced damage process in the generated idealistic and actual tested samples.

\section{Task 3: Conducting controlled freeze-thaw test and microstructure image acquisition}

Conduct controlled freeze-thaw tests on concrete and cement paste specimens in accordance to ASTM standards. The image acquisition of specimens through SEM and TXM instruments will be conducted before and after the freeze-thaw test to compare with the developed fracture model predictions.

\section{Task 4: Numerical validation of crystallization pressure mechanism for frost- induced damage in cement samples}

Conduct frost-induced damage simulation from the generated digital samples obtained from the SEM and TXM images of the cement paste specimens. The simulation results will be validated by comparing them to the fracture trends in the parent images. The validation results will provide the direction to investigate frost-induced damage in concrete samples.

\subsection{Research Objectives and Tasks}

The main objective of this doctoral study is to investigate the internal frost damage due to crystallization pore pressure in porous cement-based materials by developing computational and experimental characterization tools. After a detailed literature review, there are theories based on thermodynamic and mechanical principles that proposed the possibility of crystallization pore pressure that causes damage to the 
concrete at the sub-cooling temperature. The effect can be proven through experimental and numerical analysis. Among the techniques applied in this doctoral study are the controlled freeze-thaw tests, computational on crystallization pressure, fracture modeling by using FE modeling tools, and image data acquisition. The validations of the simulated internal frost damage model to the actual freeze-thaw samples will imply the objective of this doctoral study is achieved.

\subsubsection{Task 1: Development and validation of computational fracture models}

In order to predict the frost-induced damage in concrete, computational models need to be developed to simulate the fracture behavior in concrete. Figure 1-7 demonstrates the flow for performing Task 1. After some literature reviews, two types of FE modeling techniques were found to be suitable to simulate crack growth problems. The two FE modeling techniques are the XFEM and bilinear CZM techniques. Both techniques have been widely used to predict the detailed fracture behavior of material in the last decades. In this doctoral study, the XFEM was developed and performed using MATLAB program, whereas the bilinear CZM technique was developed and performed using ABAQUS program incorporating user subroutines.

The FE models were then constructed to simulate two typical fracture tests perform on concrete specimens, they are compact tension (CT) test and single-edgenotched beam (SEB) bending test. All the models' geometries and boundary conditions were developed or constructed in accordance to the published standards, such as the ASTM C 293-08 for the SEB test. In the CT test, the crack growth patterns and the stress intensity factors (SIFs) were analyzed using the XFEM technique. The fracture modeling 
results were then validated to the actual tested concrete specimens and theoretical analysis. The bilinear CZM technique only simulated the crack growth patterns and compared to the actual tested cement-based specimens.

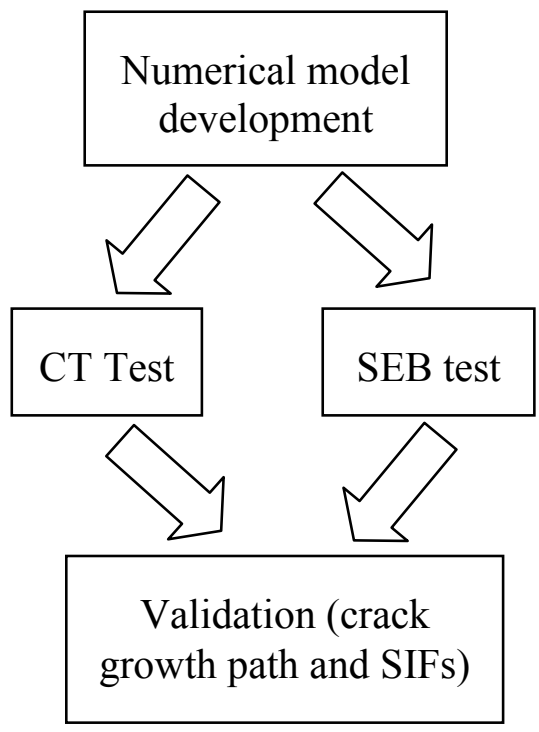

Figure 1-7: Schematic flow of Task 1: Development and validation of computational fracture models

\subsubsection{Task 2: Pore pressure calculation and damage simulation on idealized samples}

Based on Scherer's thermodynamic principles (Equations (1-4)), the ice crystallization pressure will be computed within an idealized pore system under subcooling temperatures through the developed MATLAB program. Figure 1-8 illustrates the flow of computing and simulating the ice crystallization pressure within pore system.

The pore system with idealistic 2-D geometry is developed. By assuming ice grows from capillary pores to connected nano-pores with smaller radii under sub-cooling temperatures, the generated ice crystallization pressure can be computed. In addition, the 
relative humidity and temperature is assumed to be identical for this ice crystal. The pressure generation is dependent to the curvature difference between the nano-pore and the connecting capillary pores. Thus, different magnitudes of ice crystallization pressures could be generated. By using a MATLAB program, each curvature along the pore system surfaces will be computed to determine the magnitude and location of the maximum pressure induced by the ice crystallization effect.

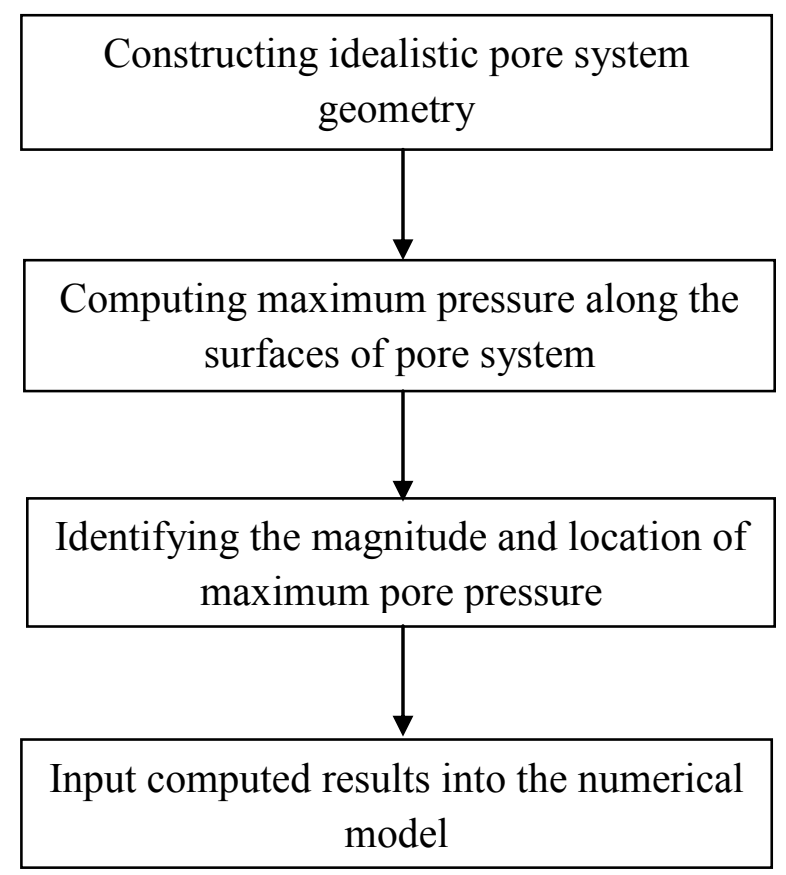

Figure 1-8: Schematic flow of Task 2: Computation and simulation of ice crystallization pressure within pore system

From the computational work, the specific location with the highest degree of stress level will be identified. The maximum stress location is essential for developing a numerical model as the XFEM and CZM require a predefined crack location. This will reduce the computational effort to simulate the crack growth as the crack location is 
predefined rather than defining several possible crack locations in the model. The idealized samples will be generated with the pore system to simulate the frost-induced damage caused by the ice crystallization pressure.

\subsubsection{Task 3: Conducting controlled freeze-thaw test and microstructure image acquisition}

One of the major tasks for this doctoral study is to conduct controlled freeze tests followed by acquiring microstructure images to investigate the fracture behavior of cement/concrete specimens. Figure 1-9 illustrates the flow chart for performing Task 3. In this doctoral study, several micro scale cylindrical cement paste specimens were fabricated. All the specimens have different designs of w/c. The specimens were freezethawed manually in the freezer for approximately 60 cycles. With the available resources and time, the SEM and TXM techniques were employed in this doctoral study to acquire imaging data for micro-damage distribution of tested specimens.

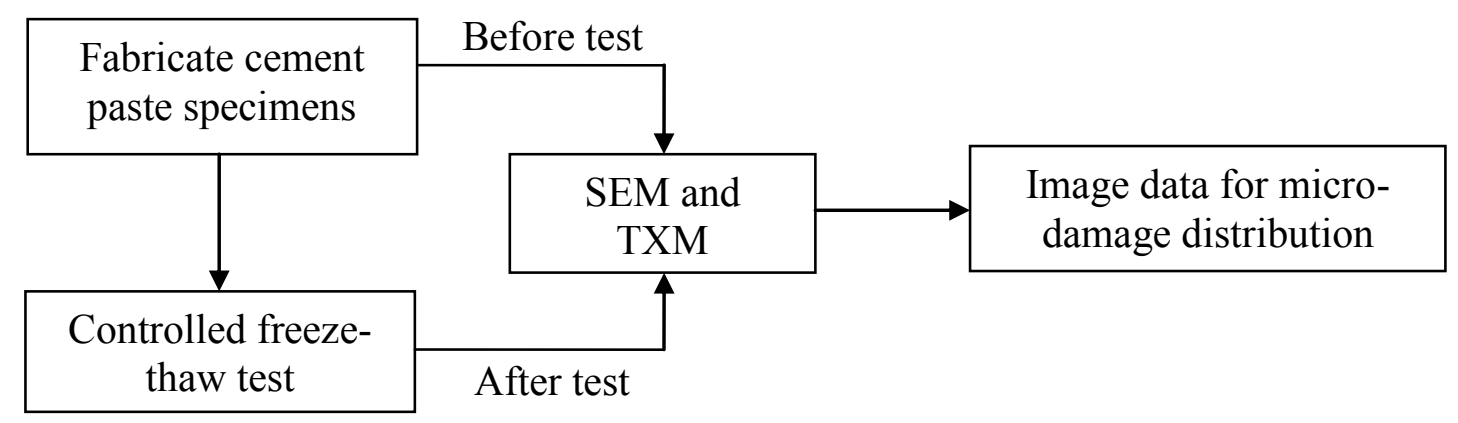

Figure 1-9: Schematic flow chart of Task 3: Performing controlled freeze test on cement paste specimens and data acquisitions, which are acquired using SEM and TXM techniques. 


\subsubsection{Task 4: Numerical validation of crystallization pressure mechanism for frost- induced damage in cement samples}

Upon completing the rapid freeze-thaw test with concrete specimens from Task 3 , the acquired imaging data are applied to conduct damage simulation with the developed computational models using the techniques developed in Tasks 1 and 2 . The digital samples are generated from the SEM and TXM images with imaging processing. The computational models are applied to efficiently simulate the crack propagation by defining the initial crack location in the digital sample. The frost-induced micro-damage simulations are conducted based on the geometry of the pore system. Firstly, the hoop stresses are calculated due to ice crystallization pressure under sub-cooling temperatures. Secondly, the crack propagations are simulated from the maximum stress level location. The simulation results from the frost-induced micro-damage model will be compared and validated with the digital sample simulation and experimental measurement. The flow chart of conducting Task 4 is as follows:

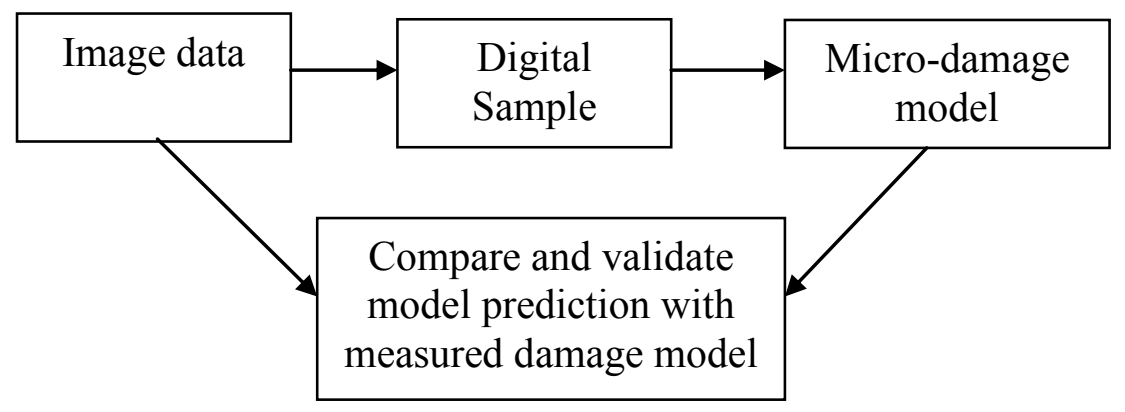

Figure 1-10: Schematic flow chart of Task 4: Performing micro-damage model validations based on the freeze-test result of concrete specimens. 


\section{CHAPTER 2 FRACTURE MODELS DEVELOPMENT FOR HETEROGENEOUS CONCRETE}

\subsection{Overview}

In this chapter, the fracture models development for heterogeneous concrete in this doctoral study will be presented. Fracture modeling has been widely applied in many studies to predict the fracture behavior in a material. The prediction will not only prevent failure of a material to occur, but also assist in creating a better design for a material. Rather than performing fracture tests on fabricated specimens, the fracture models will also help to reduce the costs of preparing specimens and running fracture tests. With these benefits, it is ideal to carry out fracture models in this doctoral study to analyze the crystallization pressure effect due to frost-induced damage on concrete specimens. After thorough literature reviews, two fracture modeling techniques are employed in this doctoral study. They are bilinear cohesive zone model (CZM) and extended finite

element method (XFEM). Both techniques will be presented with theoretical and numerical aspects, fracture simulations on uniform or idealized samples, and validation of simulation results.

\subsection{Extended Finite Element Method}

\subsubsection{Theoretical and Numerical Aspects}

The traditional FE formulation in terms of total displacement is a continuous function and is expressed as (Cook 1995; Logan 2007): 


$$
u(x)=\sum_{i=I} u_{i} N_{i}
$$

where $u$ is the displacement and $N$ is the shape function, while the subscripts $i$ represent the node index number.

In order to deal with the crack problem, the traditional FE model has been updated with discontinuity features. Recent developments such as the enriched finite element method, boundary element method, and the mesh free Galerkin method (Abdelaziz and Hamouine 2008; Belytschko and Black 1999; Belytschko et al. 1994) have solved the discontinuity issue in the FE model. However, mesh refinement is required to model the crack growth that leads to costly computational efforts. Extended finite element method (XFEM) has solved the mesh refinement (Abdelaziz and Hamouine 2008; Stolarska et al. 2001; Sukumar et al. 2001; Sukumar et al. 2004; Sukumar and Préevost 2003; Ventura et al. 2003) using the enrichment functions and level set method, as shown in the following equation:

$$
u^{h}(x)=\sum_{i \in I} u_{i} N_{i}+\sum_{j \in J} b_{j} N_{j} H(x)+\sum_{k \in K} N_{k}\left(\sum_{l=1}^{4} c_{k}^{l} F_{l}(x)\right)
$$

where $x$ is the global coordinate vector, $N_{i}$ is the traditional FE shape functions of node $i, \quad u_{i}$ is the traditional FE degrees of freedom (DOFs), $H(x)$ is the Heaviside function, $N_{j}$ is the shape function related to the Heaviside function at node $j, b_{j}$ is the additional DOFs related to the Heaviside enrichment functions (the circular nodes as shown in Figure 2-1), $F_{l}(x)$ is the crack-tip enrichment function, $N_{k}$ is the shape function related to the enriched crack tip function at node $k$, and $c_{k}^{l}$ is the additional DOFs related to the 
elastic asymptotic crack-tip enrichment functions (the square nodes as shown in Figure 2-1. In Equation (2-2), $I, J$, and $K$ represents the sets of all nodes in the mesh, Heaviside enriched nodes, and crack-tip enriched nodes, respectively. A visual representation of the enriched nodes in a crack domain is shown in Figure 2-1 (Ng and Dai 2012).

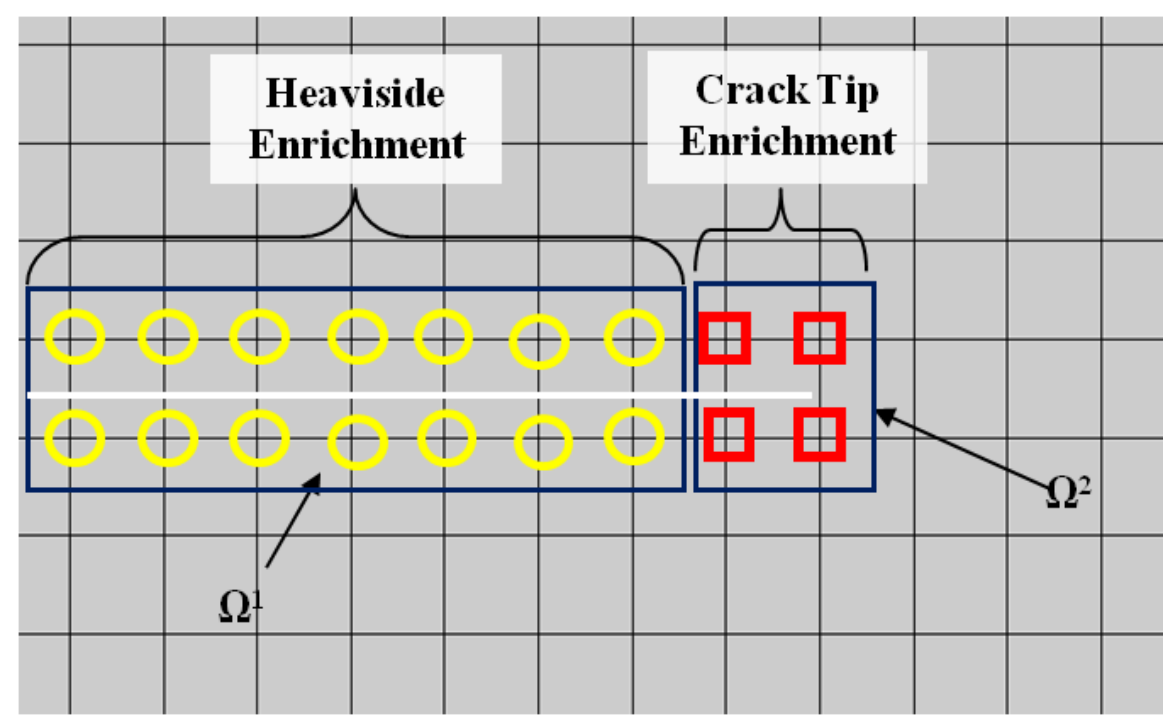

Figure 2-1: Schematic representations of Heaviside enrichment in $\Omega^{1}$ (circle) and crack tip enrichment in $\Omega^{2}$ (square) that defines a crack in the XFEM (Ng and Dai 2012) - see permission in Appendix C2 and C3.

The Heaviside function (Equation (2-3)) and crack tip enrichment function (Equation (2-4)) are as follows (Abdelaziz and Hamouine 2008; Ng and Dai 2011; 2012; Pais and Kim 2009; Stolarska et al. 2001):

$$
\begin{gathered}
H(x)=\operatorname{sign}(\phi(x))=\left\{\begin{array}{l}
-1: \phi(x)<0 \\
0: \phi(x)=0 \\
+1: \phi(x)>0
\end{array}\right. \\
{\left[F_{l}(r, \theta)\right]=\left[\sqrt{r} \sin \frac{\theta}{2}, \sqrt{r} \cos \frac{\theta}{2}, \sqrt{r} \sin \theta \sin \frac{\theta}{2}, \sqrt{r} \sin \theta \cos \frac{\theta}{2}\right]}
\end{gathered}
$$


where $\phi(x)$ is the crack surface level-set function which represents the signed (+1 and -1$)$ normal distance of a Heaviside-enriched node with respect to the crack surface, wheares the subscript $l$ represent the index number of each crack tip enrichment function froml to 4. As shown in Figure 2-2 (Ng and Dai 2012), $\psi(x)$ is the crack-tip level-set function which represents the signed distance of a crack-tip enriched node with respect to the crack tip. For the crack-tip enrichment function, $r$ and $\theta$ are the polar coordinates in the local crack-tip coordinate system. By incorporating these two functions into Equation (2-2), the enriched displacement at each node can be determined.

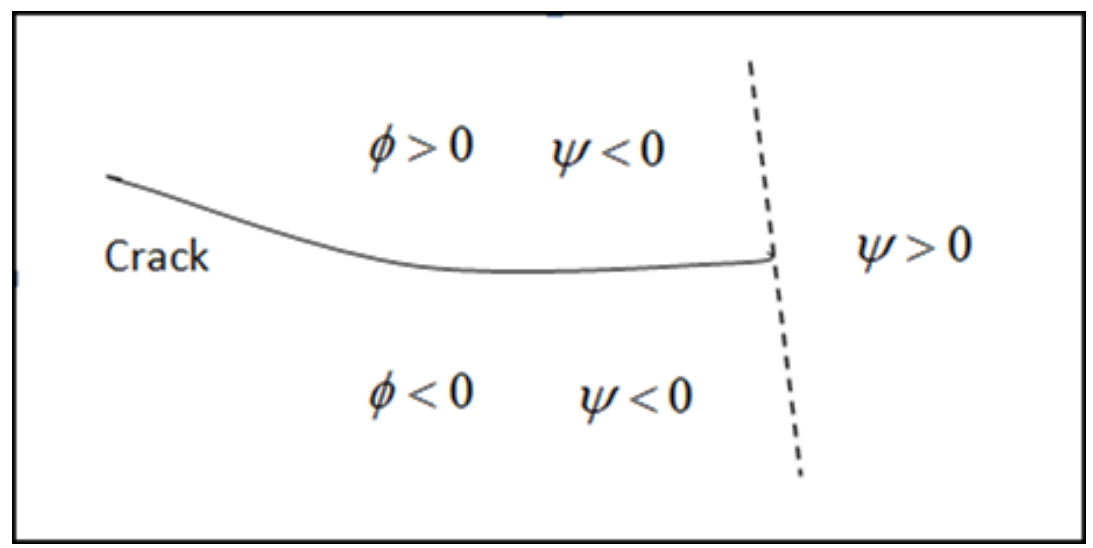

Figure 2-2: Schematic of normal and tangent level set methods that represent the geometry of crack surface and crack front boundary (Ng and Dai 2012) - see permission in Appendix $\mathrm{C} 2$ and $\mathrm{C} 3$.

For concrete, the aggregates are treated as inclusions and are defined with the inclusion enrichment function, which is given by (Abdelaziz and Hamouine 2008; Ng and Dai 2011; 2012; Pais and Kim 2009; Stolarska et al. 2001):

$$
u^{h}(x)=\sum_{i \in I} u_{i} N_{i}+\sum_{m \in M} N_{m} a_{m} F(x)
$$


The additional DOFs $a_{m}$ are defined at the nodes where the elements are split by the inclusion interface (Sukumar et al. 2001). The enrichment function $F(x)$ is taken as the following form to describe the discontinuity such as the particle debonding along the interfacial boundaries with improved convergence (Moës et al. 2003; Pais and Kim 2009):

$$
F(x)=\sum_{k \in K} N_{k}(x)\left|\zeta_{k}\right|-\left|\sum_{k} N_{k}(x) \zeta_{k}\right|
$$

where $K$ is the amount of inclusions and $\zeta_{k}(x)$ is the level set function for each inclusion. For the elliptical inclusion (Dai and $\mathrm{Ng} 2010 ; \mathrm{Ng}$ and Dai 2011; 2012), $\zeta_{k}(x)$ is given as

$$
\zeta_{k}(x)=\frac{\left[\left(x_{i}-x_{c}\right) \cos \theta+\left(y_{i}-y_{c}\right) \sin \theta\right]^{2}}{a^{2}}+\frac{\left[\left(y_{i}-y_{c}\right) \cos \theta+\left(x_{i}-x_{c}\right) \sin \theta\right]^{2}}{b^{2}}-1
$$

where $a$ and $b$ are the major and minor axes of the ellipse respectively, and $\theta$ is the orientation angle with respect to the horizontal axis. Similarly, $\zeta_{k}(x)$ for circular inclusion is given by:

$$
\zeta_{k}(x)=\sqrt{\left(x_{i}-x_{c}\right)^{2}+\left(y_{i}-y_{c}\right)^{2}}-r_{c}
$$

Figure 2-3 (Ng and Dai 2012) illustrates inclusions in the XFEM model with the inclusion enrichment (red circles) are defined along the inclusion boundaries. 


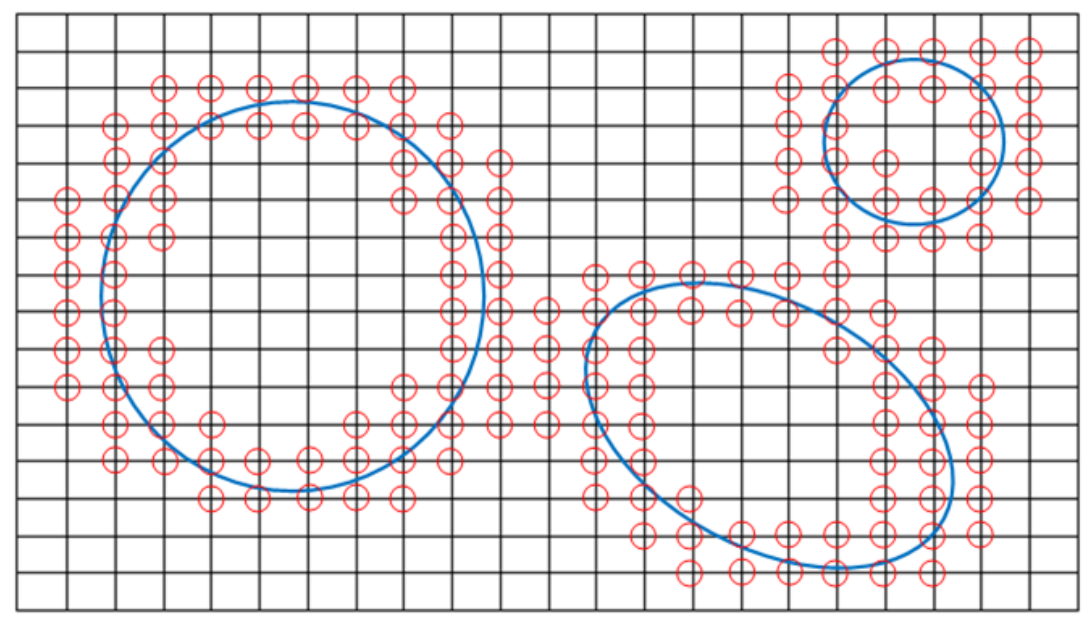

Figure 2-3 Schematic representation of inclusion enrichments (red circles) ( $\mathrm{Ng}$ and Dai 2012) - see permission in Appendix C3.

To simulate the crack propagation direction, the maximum energy release rate will be applied as the fracture criterion. For a mixed mode case, the energy release rate, $J$ is a function of the stress intensity factor (SIF), $K$ and effective modulus, $E_{\text {eff }}$ as below:

$$
J=\frac{K_{I}^{2}+K_{I I}^{2}}{E_{e f f}}
$$

The effective modulus $E_{\text {eff }}$ takes the following form for the conditions of plane stress or plane strain:

$$
E_{\text {eff }}= \begin{cases}E & , \text { Plane Stress } \\ \frac{E}{1-v^{2}} \quad, & \text { Plane Strain }\end{cases}
$$

where $E$ is the Young's modulus and $v$ is the Poisson's ratio of the cement material.

From the fracture mechanics theory, the J-integral is given as the following equation: 


$$
J=\int_{\Gamma}\left[W \delta_{1 j}-\sigma_{i j} \frac{\partial u_{i}}{\partial x_{1}}\right] \eta_{j} d \Gamma
$$

where $W$ is the strain energy density, $\eta$ is normal vector to the integration curve $\Gamma, \sigma$ is the Cauchy stress tensor, and $u$ is the displacement vector.

It has been proven that using the two-state J-integral approach can efficiently compute the SIFs around the crack tip (Jeon et al. 2008). Each state has stress $\left(\sigma_{i j}\right)$, strain $\left(\varepsilon_{i j}\right)$, and displacement $\left(u_{i}\right)$ as follows (Moës et al. 1999; Ng and Dai 2011; 2012; Pais and Kim 2009):

- State $1,\left(\sigma_{i j}^{(1)}, \varepsilon_{i j}^{(1)}, u_{i}^{(1)}\right):$ It refers to the present state with the XFEM solution

- State $2,\left(\sigma_{i j}^{(2)}, \varepsilon_{i j}^{(2)}, u_{i}^{(2)}\right)$ : It refers to an auxiliary state which is selected as the elastic asymptotic field for Modes I or/and II referred to (Williams 1957).

By superimposing the two states into the general J-integral Equation (2-11), the two-state J-integral is obtained as:

$$
J^{(1+2)}=\int_{\Gamma}\left[\frac{1}{2}\left(\sigma_{i j}^{(1)}+\sigma_{i j}^{(2)}\right)\left(\varepsilon_{i j}^{(1)}+\varepsilon_{i j}^{(2)}\right) \delta_{i j}-\left(\sigma_{i j}^{(1)}+\sigma_{i j}^{(2)}\right) \frac{\partial\left(u_{i}^{(1)}+u_{i}^{(2)}\right)}{\partial x_{1}}\right] \eta_{j} d \Gamma
$$

The J-integral in Equation (2-12) can be divided with two separate J-integrals of the present state 1 and the auxiliary state 2 and an interaction term, $I^{(1,2)}$ as following:

$$
J^{(1+2)}=J^{(1)}+J^{(2)}+I^{(1,2)}
$$

and the interaction term $I^{(1,2)}$ takes the form as: 


$$
I^{(1,2)}=\int_{\Gamma}\left[W^{(1,2)} \delta_{1 j}-\sigma_{i j}^{(1)} \frac{\partial\left(u_{i}^{2}\right)}{\partial x_{1}}-\sigma_{i j}^{(2)} \frac{\partial\left(u_{i}^{1}\right)}{\partial x_{1}}\right] n_{j} d \Gamma
$$

where $W^{(1,2)}$ is the interaction strain energy density as:

$$
W^{(1,2)}=\sigma_{i j}^{(1)} \varepsilon_{i j}^{(2)}=\sigma_{i j}^{(2)} \varepsilon_{i j}^{(1)}
$$

By superimposing two states with $K_{I}=K_{I}^{(1)}+K_{I}^{(2)}$ and $K_{I I}=K_{I I}^{(1)}+K_{I I}^{(2)}$, the J-integral Equation (2-9) can be formulated and rearranged as:

$$
J^{(1+2)}=J^{(1)}+J^{(2)}+\frac{2}{E_{e f f}}\left(K_{I}^{(1)} K_{I}^{(2)}+K_{I I}^{(1)} K_{I I}^{(2)}\right)
$$

By equating Equation (2-13) with Equation (2-16), the interaction integral becomes:

$$
I^{(1,2)}=\frac{2}{E_{\text {eff }}}\left(K_{I}^{(1)} K_{I}^{(2)}+K_{I I}^{(1)} K_{I I}^{(2)}\right)
$$

The SIFs of the present State 1 can then be determined with the interaction integral. By setting the elastic asymptotic fields of State 2 as: $K_{I}^{(2)}=1$ and $K_{I I}^{(2)}=0$, the stress intensity factor $K_{I}$ of the State 1 can be solved as:

$$
K_{I}=\frac{1}{2} E_{e f f} I^{(\text {ModeI })}
$$

Similarly, the stress intensity factor $K_{I I}$ of the State 1 can be solved in the similar manner by setting $K_{I}^{(2)}=0$ and $K_{I I}^{(2)}=1$, as the following term:

$$
K_{I I}=\frac{1}{2} E_{e f f} I^{(\text {ModeII })}
$$


To model the crack propagation, the fracture criterion of the maximum energy release rate and proportional crack growth law are applied to determine the crack growth path. The energy release rate $(G)$ measures the released energy for an increment of crack growth and takes the same formula as the J-integral (Anderson 1995):

$$
G=\frac{K_{I}^{2}+K_{I I}^{2}}{E_{\text {eff }}}
$$

In the tailored XFEM, the numerical scheme for modeling the interaction between crack propagation and particles is illustrated in Figure 2-4 (Ng and Dai 2012). For each crack increment $\Delta \alpha$, the crack propagation with the maximum $G$ is determined by using a predefined search distance $d_{s}$ and serial of kink angles $\beta$ (Bush 1997). In the tailored XFEM, the kink angles are selected in a range from $-44^{\circ}$ to $+44^{\circ}$ with an interval of $4^{\circ}$. Assuming that the effective stress exceeds the material fracture strength in the XFEM, the crack increment $\Delta \alpha$ is chosen about 1.2 times the element length. To simulate the interaction between crack propagation and particles, the search path associated with the kink angle $\beta$ occupies a five-element distance away from the crack tip. When the crack approaches or passes an elastic particle, the shielding effects that lead to the reduction of energy release rate $G$ were shown by Li and Chudnovski (Li and Chudnovsky 1993). In the tailored XFEM, this effect was implemented by setting the lower $G$ within the interfacial zones compared to that of the cement. By applying the search numerical scheme and the maximum $G$ criterion, the crack propagates within or near the interfacial zones without penetrating into them. This numerical scheme was used to simulate the interfacial transition zones by addressing the shielding effects in the cementitious materials. 


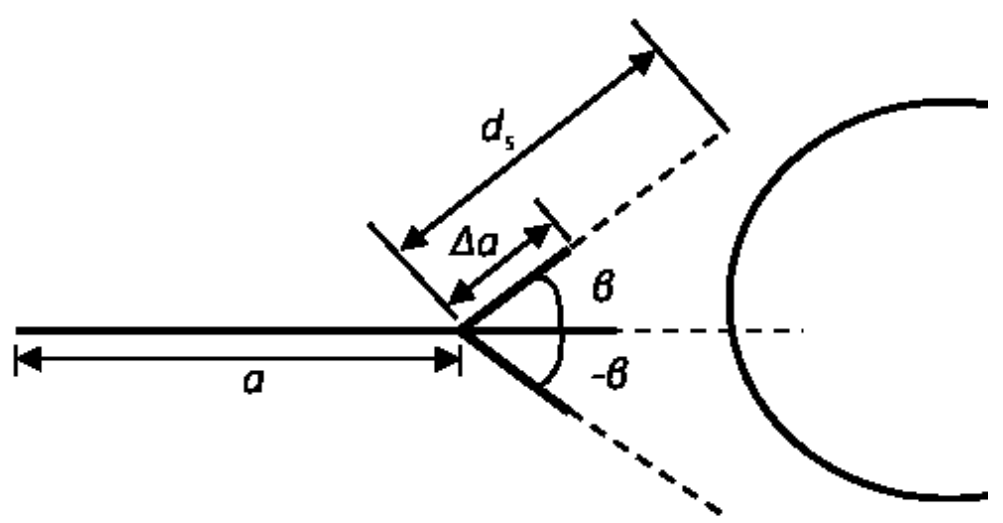

Figure 2-4: Numerical scheme for modeling the interaction between crack propagation and particles: for each crack increment $\Delta \alpha$, the crack propagation with the maximum energy release rate is determined by using a predefined search distance $d_{\mathrm{s}}$ and a number of kink angles $\beta$ (Ng and Dai 2012) - see permission in Appendix C3.

\subsubsection{Fracture property prediction on uniform samples}

\subsubsection{Compact tension test}

The Compact Tension (CT) test has been widely used to evaluate the fracture properties of material specimens using the rectangular geometry. The test uses a rectangular specimen with a single-edge notch. Two symmetric holes are placed in the specimen: one hole is applied with the tensile loading and the other one is fixed as shown in the Figure 2-5 (Ng and Dai 2012). For validation, the CT fracture test will be modeled to predict the crack growth propagation. The computed stress intensity factors (SIFs) also will be compared to the theoretical SIFs which is given as (Anderson 1995):

$$
K_{I}=\frac{f\left(\frac{a}{W}\right) P}{B \sqrt{W}}
$$

in which, 
$f\left(\frac{a}{W}\right)=\frac{2+\frac{a}{W}}{\left(1-\frac{a}{W}\right)^{3 / 2}}\left[0.886+4.64\left(\frac{a}{W}\right)-13.32\left(\frac{a}{W}\right)^{2}+14.72\left(\frac{a}{W}\right)^{3}-5.60\left(\frac{a}{W}\right)^{4}\right]$

where $B$ is the specimen thickness, $a$ is the distance between the midpoint of circular hole and the crack end, $W$ is the distance between the midpoint of circular hole and the crack edge, and $P$ is the applied load. Figure 2-5 ( $\mathrm{Ng}$ and Dai 2012) also illustrates the geometries and boundary conditions of the CT fracture test.

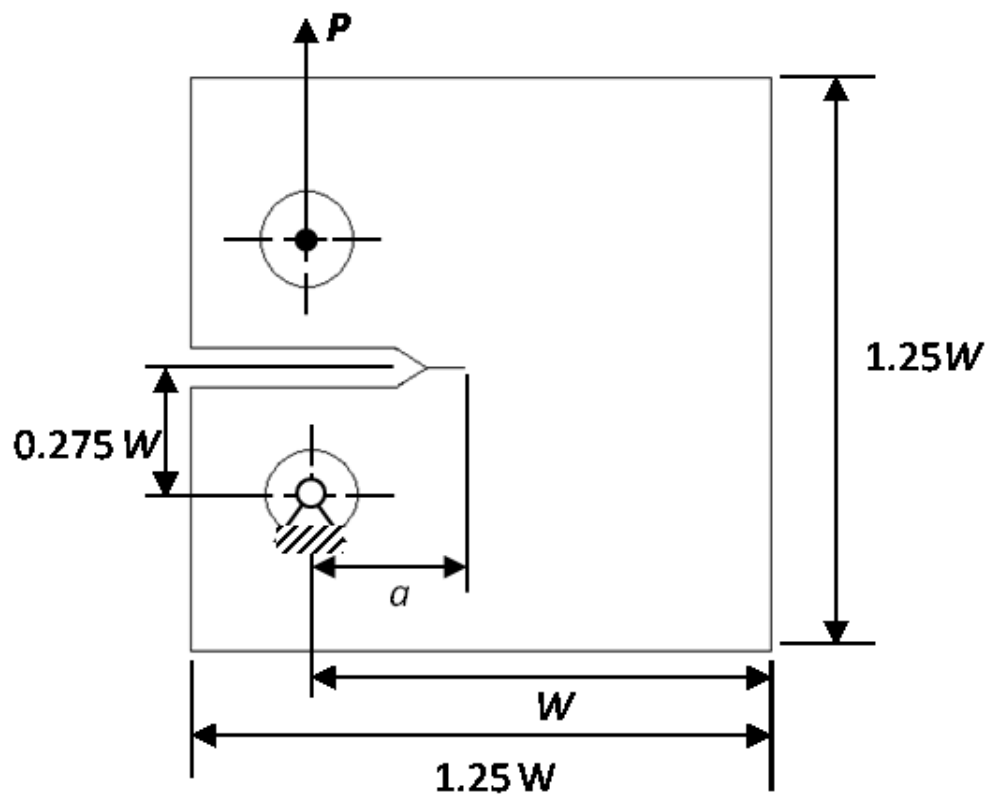

Figure 2-5: Schematic representation of CT fracture test (Ng and Dai 2012) - see permission in Appendix C3, with standard dimension (Anderson 1995; Ng and Dai 2011).

The geometry dimensions were selected to meet the standard test requirements (Sanford 2003) of $W=40 \mathrm{~m}, B=W / 2=20 \mathrm{~m}$ and the initial crack length $a_{0}=10 \mathrm{~m}$. 
The tensile force $P=1.0 \times 10^{10} \mathrm{~N}$ is applied at one hole. For the XFEM simulation, the elastic modulus of cementitious materials was chosen as $30 \mathrm{GPa}$. The XFEM fracture simulation was conducted by using four-node quadrilateral elements under the plane stress condition. The fracture simulation was conducted to grow the crack from the initial notch length $a=a_{0}$ to a final length $a=3.0 a_{0}$.

The finite element mesh with crack enrichments and the $\sigma_{y y}$ stress field were given in Figure 2-6 ( $\mathrm{Ng}$ and Dai 2012). Figure 2-6(a) and (b) illustrate the crack propagation at a crack length $a=1.4 a_{0}$. And Figure 2-6(c) and (d) show the crack propagation for the final crack length $a=3.0 a_{0}$. The stress intensity factor $K_{I}$ was predicted from the XFEM simulation with Equations (2-16) to (2-18). Using Equations (2-21) and (2-22) from the linear fracture mechanics analysis, the theoretical $K_{I}$ was calculated with respect to the propagated crack length, $a$. In the process of the crack propagation, the predicted and theoretical $K_{I}$ were compared as listed in the Table 2-1 ( $\mathrm{Ng}$ and Dai 2012). The relative average error was calculated about $1 \%$ during the crack propagation, therefore the XFEM simulation results were validated. The validated XFEM was then applied to study the crack propagation and fracture properties with idealized samples and generated digital samples in the following sections. 


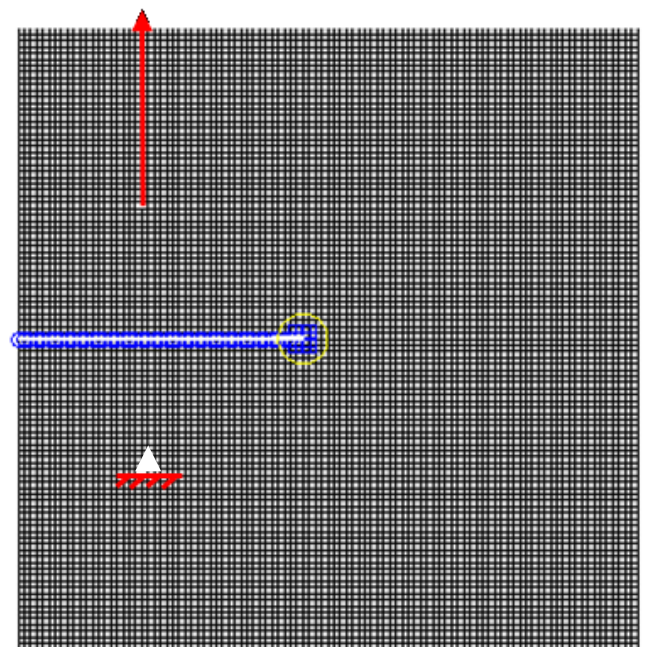

(a)

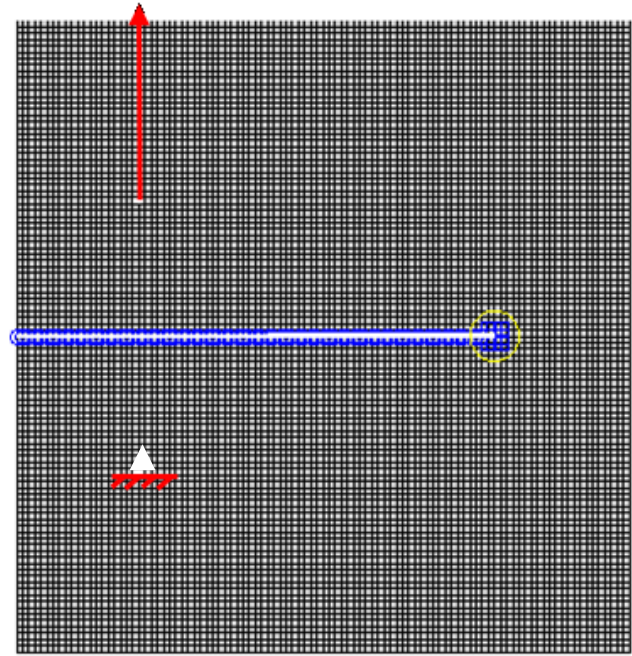

(c)

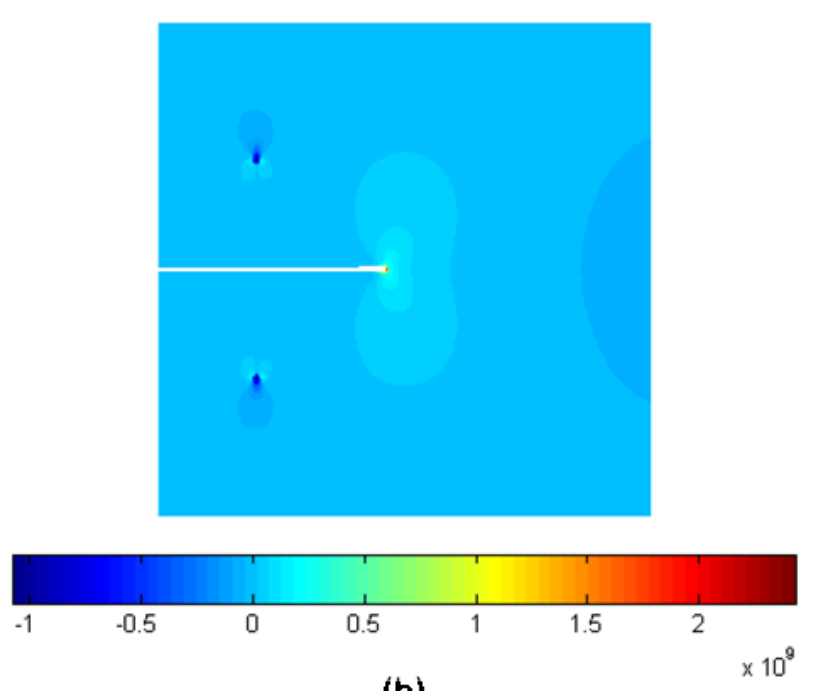

(b)

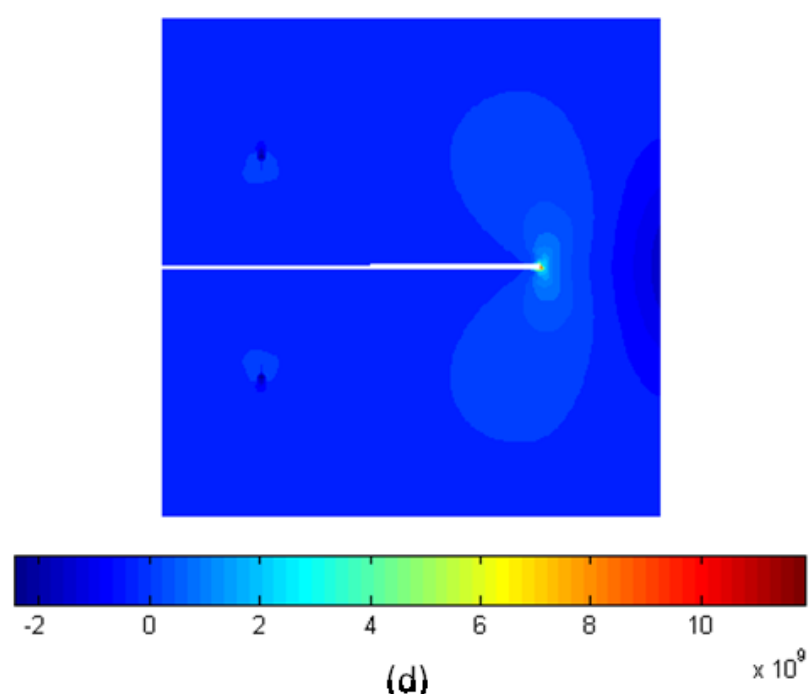

Figure 2-6: XFEM simulation of CT testing with two crack growth length $a$, (a) the mesh plot with crack enrichments for $a=1.4 a_{0}$, (b) the contour plot of $\sigma_{y y}$ stress for $a=1.4 a_{0}$, (c) the mesh plot with crack enrichments for $a=3.0 a_{0}$, (d) the contour plot of $\sigma_{y y}$ stress for $a=3.0 a_{0}(\mathrm{Ng}$ and Dai 2012) - see permissions in Appendices C2 and C3. 
Table 2-1: Comparison of calculated and theoretical stress intensity factor $K_{I}$ from XFEM simulation and fracture mechanics analysis (Ng and Dai 2012)

\begin{tabular}{|c|c|c|c|}
\hline \multirow{2}{*}{$\begin{array}{c}\text { Crack Length, } \\
a(\mathrm{~m})\end{array}$} & \multicolumn{2}{|c|}{$\boldsymbol{K}_{\boldsymbol{I}}(P a \sqrt{m})$} & \multirow{2}{*}{$\begin{array}{c}\text { Relative Error } \\
\text { Percentage }\end{array}$} \\
\hline & Calculated & Theoretical & \\
\hline 12 & $4.29 \mathrm{E}+08$ & $4.10 \mathrm{E}+08$ & 4.47 \\
\hline 13 & $4.53 \mathrm{E}+08$ & $4.38 \mathrm{E}+08$ & 3.28 \\
\hline 14 & $4.78 \mathrm{E}+08$ & $4.67 \mathrm{E}+08$ & 2.32 \\
\hline 15 & $5.06 \mathrm{E}+08$ & $4.98 \mathrm{E}+08$ & 1.57 \\
\hline 16 & $5.37 \mathrm{E}+08$ & $5.31 \mathrm{E}+08$ & 0.99 \\
\hline 17 & $5.71 \mathrm{E}+08$ & $5.67 \mathrm{E}+08$ & 0.58 \\
\hline 18 & $6.08 \mathrm{E}+08$ & $6.06 \mathrm{E}+08$ & 0.30 \\
\hline 19 & $6.51 \mathrm{E}+08$ & $6.50 \mathrm{E}+08$ & 0.13 \\
\hline 20 & $6.99 \mathrm{E}+08$ & $6.98 \mathrm{E}+08$ & 0.06 \\
\hline 21 & $7.53 \mathrm{E}+08$ & $7.52 \mathrm{E}+08$ & 0.07 \\
\hline 22 & $8.15 \mathrm{E}+08$ & $8.14 \mathrm{E}+08$ & 0.14 \\
\hline 23 & $8.87 \mathrm{E}+08$ & $8.85 \mathrm{E}+08$ & 0.26 \\
\hline 24 & $9.71 \mathrm{E}+08$ & $9.67 \mathrm{E}+08$ & 0.40 \\
\hline 25 & $1.07 \mathrm{E}+09$ & $1.06 \mathrm{E}+09$ & 0.55 \\
\hline 26 & $1.18 \mathrm{E}+09$ & $1.18 \mathrm{E}+09$ & 0.69 \\
\hline 27 & $1.32 \mathrm{E}+09$ & $1.31 \mathrm{E}+09$ & 0.81 \\
\hline 28 & $1.49 \mathrm{E}+09$ & $1.48 \mathrm{E}+09$ & 0.90 \\
\hline 29 & $1.69 \mathrm{E}+09$ & $1.68 \mathrm{E}+09$ & 0.93 \\
\hline 30 & $1.95 \mathrm{E}+09$ & $1.93 \mathrm{E}+09$ & 0.91 \\
\hline
\end{tabular}

\subsubsection{Single edge-notched beam (SEB) bending tests}

The SEB test method has been widely used to determine the fracture properties of material specimens. This single-edge notched beam was loaded with a mid-point compression force on top of the beam. A fixed support and a roller support were applied at two near-end locations of the beam, as shown in Figure 2-7(a) (Ng and Dai 2011). The geometry dimensions were selected to meet the standard ASTM C 293-08 SEB test requirements. The determined SIFs of the notched crack under the SEB test can be utilized to evaluate the fracture toughness of the material specimens. 
To verify the developed XFEM, the XFEM prediction on SIFs was compared with the fracture mechanics analysis of a SEB test specimen. The theoretical Mode I stress intensity factor $K_{I}$ of a SEB tested specimen is similar to the CT test (Equation (2-21)). Whereas, the function $f\left(\frac{a}{W}\right)$ is given by (Anderson 1995):

$$
f\left(\frac{a}{W}\right)=\frac{3 \frac{S}{W} \sqrt{\frac{a}{W}}}{2\left(1+2 \frac{a}{W}\right)\left(1-\frac{a}{W}\right)^{3 / 2}}\left[1.99-\left(\frac{a}{W}\right)\left(1-\frac{a}{W}\right)\left\{2.15-3.93\left(\frac{a}{W}\right)+2.7\left(\frac{a}{W}\right)^{2}\right\}\right]
$$

where $B=$ specimen thickness, $S=$ distance between two support points, $a=$ initial crack length, $W=$ specimen width, and $P=$ applied load.

The XFEM simulation was conducted on an open-mode SEB beam test shown in Figure 2-7(b) and (c). The elastic modulus and Poisson's ratio were chosen as $E_{m}=80 \mathrm{GPa}$ and $v_{a}=0.3$ for aggregates, and $E_{m}=15 \mathrm{GPa}, v_{m}=0.3$ for the matrix phase, respectively ( $\mathrm{Ng}$ and Dai 2011). The notched crack with an initial length of $a_{0}=20 \mathrm{~mm}$ and beam thickness of $B=75 \mathrm{~mm}$, were defined. The crack was propagated straight towards the top edge of the beam with several propagation iterations. The XFEM simulation results include the calculated crack SIFs, mesh plot with enriched elements, and stress contours. Equation (2-9) was applied for computing the Mode I stress intensity factor $K_{I}$ values in the XFEM simulation.

The $\sigma_{y y}$ stress field was given for the initial length $a_{0}$ in Figure 2-7(b). When the crack propagated up to the length of $a=2.5 a_{0}$, the $\sigma_{y y}$ stress contour was updated as shown in Figure 2-7(c). The contour shows that the stress intensity level has the highest 
value at the crack tip. The XFEM calculated SIFs at each crack propagation iteration. These calculated SIFs were compared with the fracture mechanic analysis with Equations (2-21) and (2-23) as listed in Table 2-2 (Ng and Dai 2011). The XFEM prediction was validated since the calculated SIFs have $2.88 \%$ relative average error compared to the analytical results from the fracture mechanics. The validated XFEM was then applied to study the fracture properties and micro-damage evolution in the following section for the lab-tested stone-based material specimens.

Table 2-2: Comparison of $K_{I}$ of a SEB fracture test between analytical results from XFEM simulation and theoretical values from fracture mechanics analysis (Ng and Dai 2011).

\begin{tabular}{|c|c|c|c|}
\hline \multirow{2}{*}{$\begin{array}{c}\text { Crack Length, } \\
\boldsymbol{a}(\mathbf{m})\end{array}$} & \multicolumn{2}{|c|}{$\boldsymbol{K}_{\boldsymbol{I}}(\boldsymbol{k P a} \sqrt{\boldsymbol{m}})$} & Relative Error \\
\cline { 2 - 4 } & Analytical & Theoretical & Percentage (\%) \\
\hline 22 & 51.81 & 53.53 & 3.31 \\
\hline 24 & 54.65 & 56.38 & 3.17 \\
\hline 26 & 57.58 & 59.34 & 3.05 \\
\hline 28 & 60.64 & 62.45 & 2.97 \\
\hline 30 & 63.85 & 65.72 & 2.92 \\
\hline 32 & 67.25 & 69.18 & 2.88 \\
\hline 34 & 70.84 & 72.87 & 2.86 \\
\hline 36 & 74.68 & 76.82 & 2.86 \\
\hline 38 & 78.80 & 81.05 & 2.86 \\
\hline 40 & 83.23 & 85.61 & 2.87 \\
\hline 42 & 88.01 & 90.54 & 2.87 \\
\hline 44 & 93.21 & 95.89 & 2.88 \\
\hline 46 & 98.86 & 101.70 & 2.87 \\
\hline 48 & 105.05 & 108.06 & 2.86 \\
\hline 50 & 111.85 & 115.02 & 2.83 \\
\hline 52 & 119.35 & 122.69 & 2.79 \\
\hline 54 & 127.67 & 131.17 & 2.74 \\
\hline 56 & 136.93 & 140.59 & 2.67 \\
\hline 58 & 147.30 & 151.11 & 2.59 \\
\hline
\end{tabular}




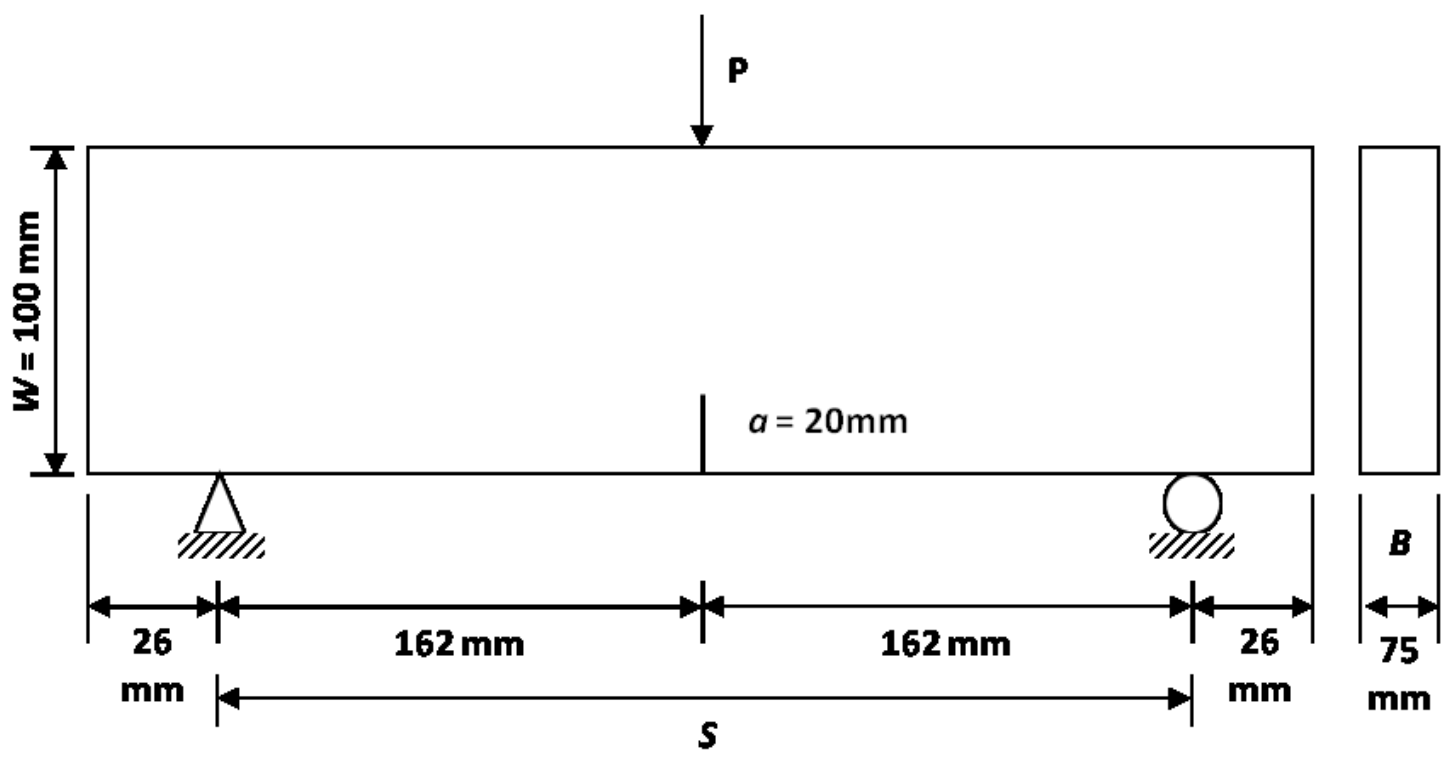

(a)

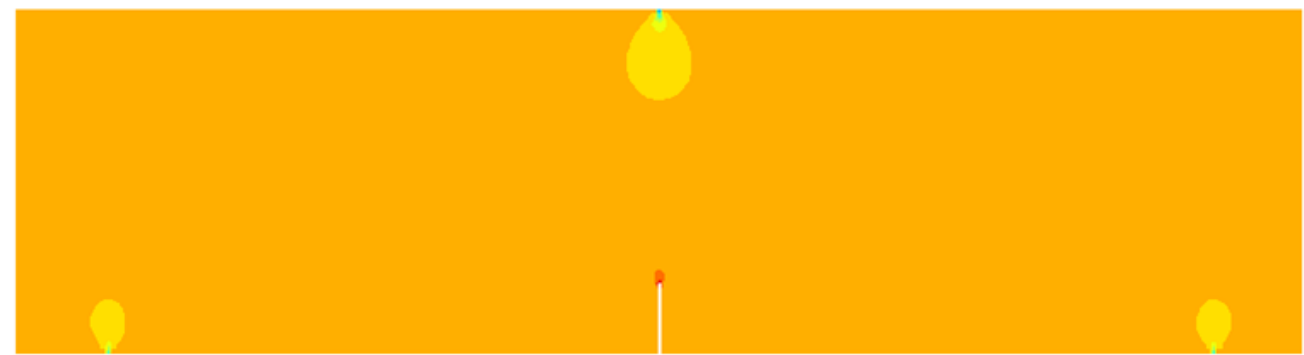

(b)

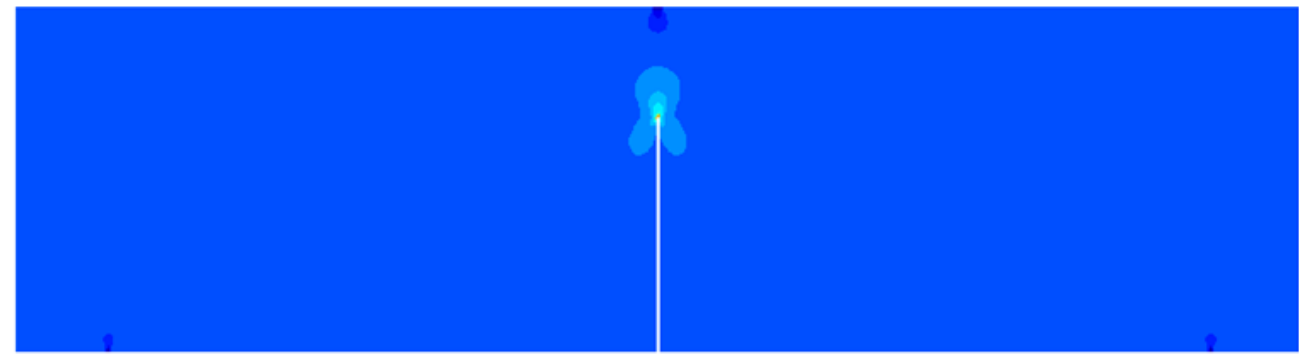

(c)

Figure 2-7: Validation simulation with an open-mode middle-notched SEB fracture test on a homogeneous sample: a) standard beam dimensions with boundary conditions, b) stress contour with the initial crack length, $a_{0}=20 \mathrm{~mm}$, and (c) $\sigma_{y y}$ contour plot with the crack length, $a=2.5 a_{0}(\mathrm{Ng}$ and Dai 2011) - see permission in Appendix C1. 
By offsetting the notched crack, the SEB test can be used to characterize the mixed-mode fracture properties. The mixed-mode SEB tests can further extend the fracture study with the open-mode CT tests. As shown in Figure 2-8(a) (Ng and Dai 2012), the test geometry includes a beam specimen with an offset single-edge notch. The bottom fixed and roller supports were applied on the left and right sides respectively. The top point force was imposed in the middle of the specimen. The XFEM fracture simulation was conducted on an offset-notched homogeneous sample by using four-node quadrilateral elements under plain stress conditions. The detailed dimensions of the simulated notched beam were also indicated in the Figure 2-8(a). The elastic modulus of cementitious materials was chosen as $30 \mathrm{GPa}$.

The XFEM prediction of the mixed-mode SEB test are shown in Figure 2-8(b) and (c). Figure 2-8(b) shows the finite element mesh with the crack path and crack enrichments. It can be clearly observed that the crack propagated across the elements. This tailored XFEM employed the maximum $G$ criterion to determine the crack propagation path. For this mixed-mode fracture test, the predicted crack propagation matched well with a cohesive fracture modeling by Song et al. (Song et al. 2006a). Furthermore, the XFEM fracture model has advantages in efficiently predicting the complex crack path by eliminating mesh refinements that are required in the cohesion fracture model (Song et al. 2006a). The $\sigma_{y y}$ stress contour with the crack path was shown in Figure 2-8(c). In another study, the mixed-mode crack path obtained with the stress fracture criteria is identical with this fracture energy-based simulation. 


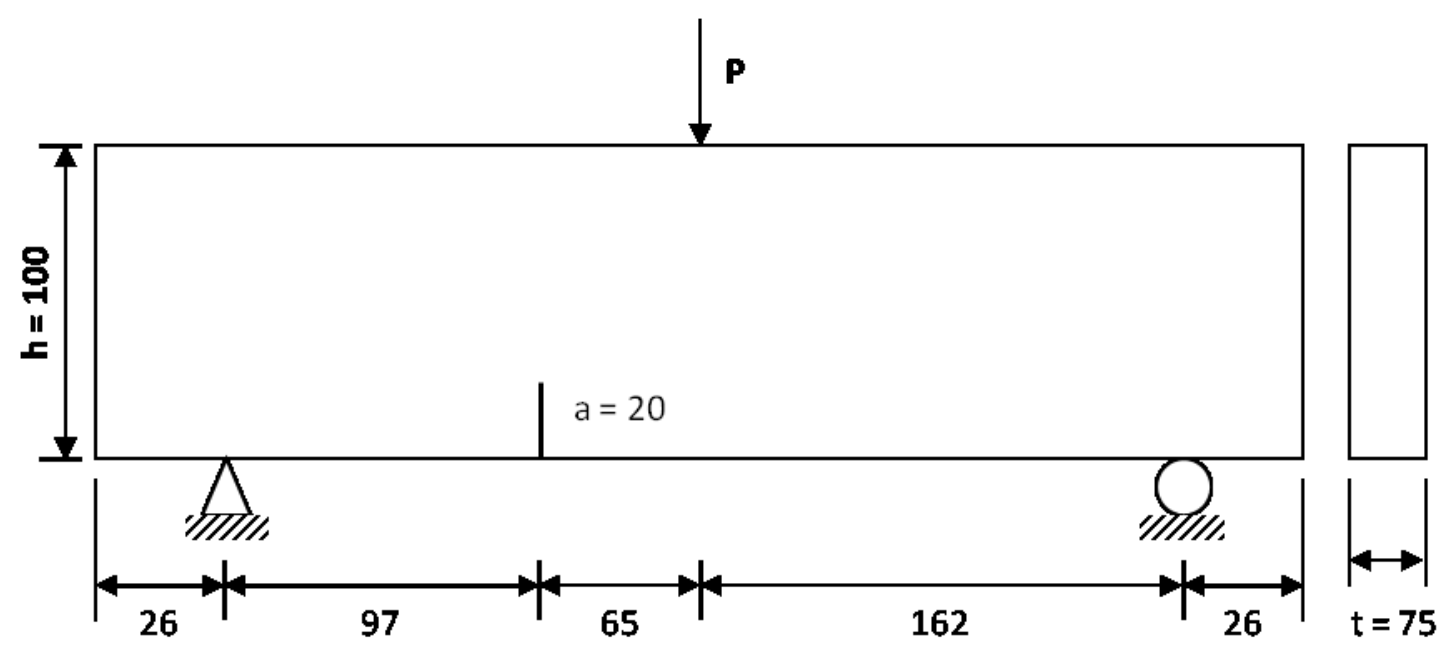

(a)

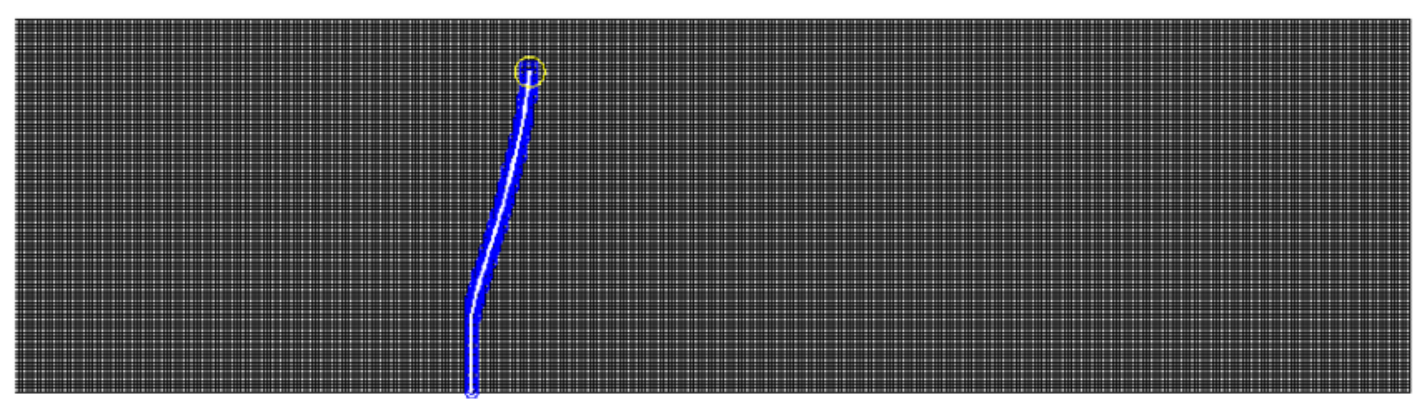

(b)

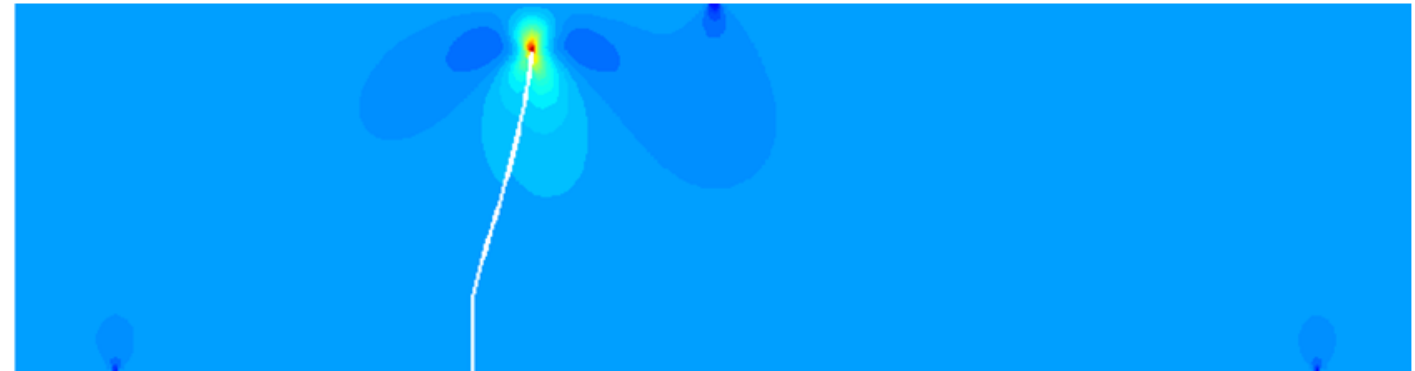

(c)

Figure 2-8: Singe-edge notched beam simulation with a uniform cement sample, (a) mixed-mode test geometry with offset notch, (b) element mesh with crack path and enrichment, (c) $\sigma_{y y}$ stress contour with crack path ( $\mathrm{Ng}$ and Dai 2012) - see permission in Appendix C3. 


\subsubsection{Fracture simulations on idealized cementitious sample}

To predict the crack propagation with the cement-particle system, the particle inclusion enrichment functions (Equations (2-6) and (2-7)) were defined to represent the discontinuity of particle debonding along the interfacial boundaries. At the initial stage, the effect of particle shapes and orientations were investigated. Upon validating the feasibility of XFEM in modeling the crack growth problem with inclusions, lab-prepared concrete specimens were modeled from mechanical tests, such as SEB bending test.

\subsubsection{XFEM CT test fracture simulation with idealized cementitious samples}

The XFEM fracture simulation was conducted with idealized cementitious samples under the same CT testing configuration as the previous section. These idealized samples were composed of circular and elliptical particles as shown in Figure 2-9 to Figure 2-11 (Ng and Dai 2012). This study considered the effects of particle location, orientation and shape factor. In the XFEM simulation, the particles were treated as inclusions and thus the enrichment function was applied as Equation (2-7). The interaction between the crack propagation and particles was simulated with the search numerical scheme and the maximum $G$ fracture criterion. For the XFEM simulation, the elastic modulus of cement paste and particles were defined as $15 \mathrm{GPa}$ and $80 \mathrm{GPa}$ respectively. These XFEM fracture simulation was conducted by using four-node quadrilateral elements with identical dimensions with a mesh size of $200 \times 200$. Plane stress condition with a uniform thickness $B=20 \mathrm{~m}$ was applied in the model.

In order to explore the effect of particle location on the crack propagation, the predicted fracture paths were compared with two idealized samples composed of circular 
particles. As shown in Figure 2-9, the only difference between these two samples is the relative location of a circular particle A with respect to the crack line. Figure 2-9(a) and (b) show the element mesh with crack path and the $\sigma_{y y}$ stress contour for the particle A located at $e=3 d / 4$, where $e$ is the normal distance of the particle center with respect to the crack line and $d$ is the particle diameter. Figure 2-9 (c) and (d) are for the case that the circular particle A located at $\mathrm{e}=d / 4$. In both cases, the crack propagated along the particle boundaries with the maximum $G$ criterion and the search numerical scheme. These results also show the shielding effects within the interfacial transition zones of the cementitious materials.

This study also investigated the effects of the orientation $\theta$ of particles on crack propagation by using the samples with the ellipse inclusions as shown in Figure 2-10. The XFEM fracture simulation was conducted with the same CT testing configuration and the finite element mesh as previous. Figure 2-10 shows the XFEM fracture simulation of CT samples with two particle orientations $\left(-20^{\circ}\right.$ and $\left.-45^{\circ}\right)$. Both simulation results show how the predicted crack propagates tangentially to particle B (as indicated in Figure 2-10) and approached the boundary zone of the next particle. These simulation results again show the shielding effects of the interfacial transition zones in the cementitious materials.

The effect of the particle shape on the crack evolution was also studied with two idealized cementitious material samples shown in Figure 2-11. The only difference between these two samples is the particle shape factor (SF) that is defined as the ratio of the largest and the smallest dimensions. The other micro-parameters including the particle size (largest dimension) and orientation $\left(-20^{\circ}\right)$ are identical. Figure 2-11(a) and 
(b) show the element mesh with enrichments and $\sigma_{y y}$ stress contour for the shape factor of 2.67. The crack propagated tangentially to these two particles. When the shape factor increased to 1.33 as shown in Figure 2-11(c) and (d), the crack path was tangential to the first particle $\mathrm{C}$ and approached to the next particle. 


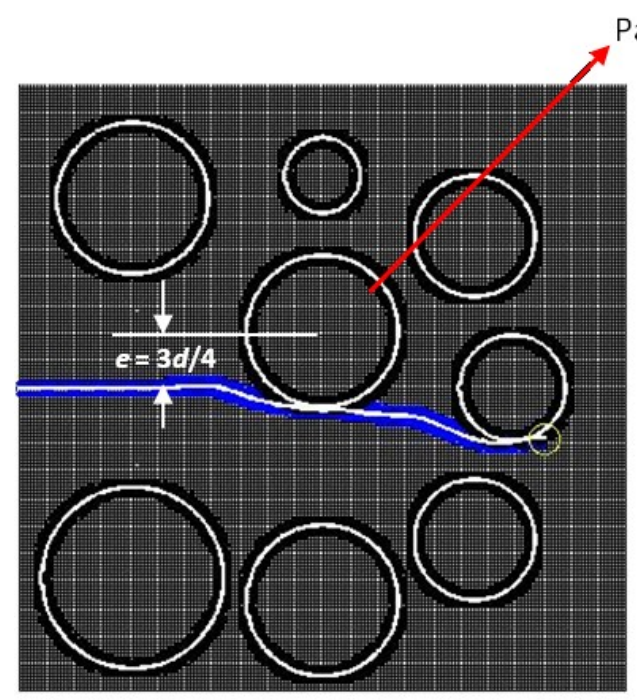

(a)
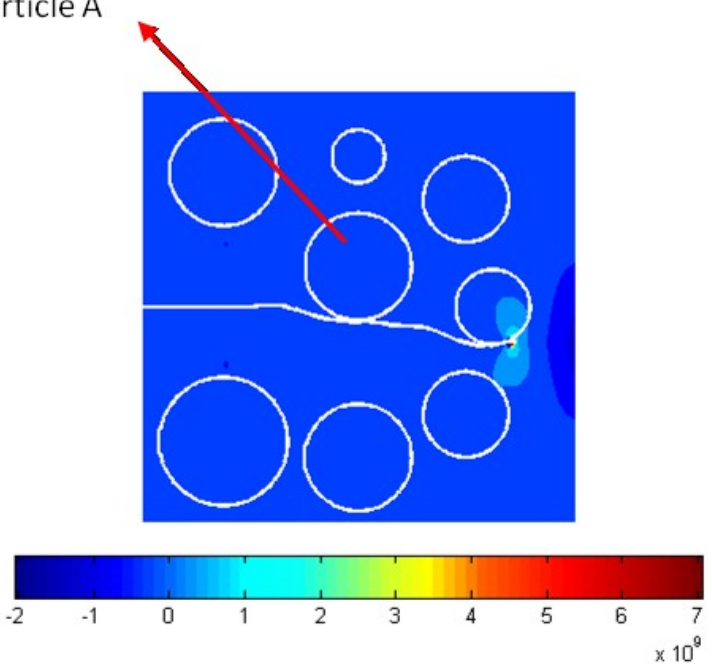

(b)

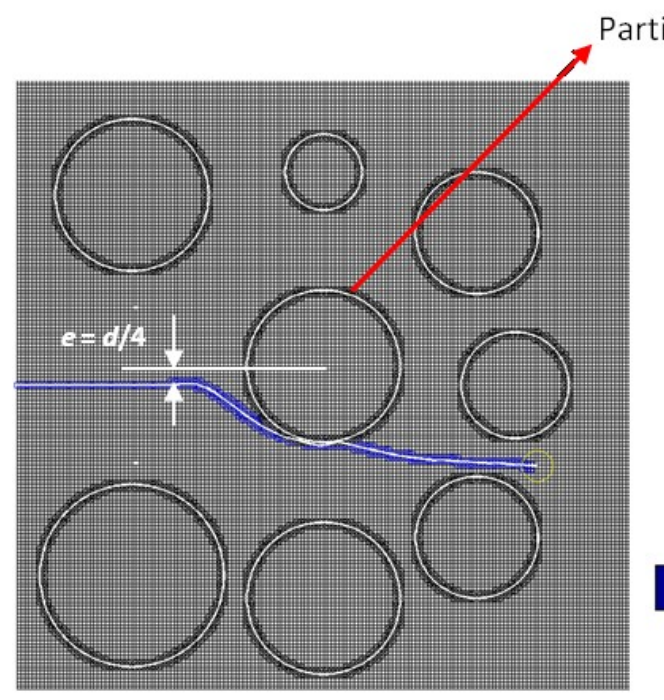

(c)

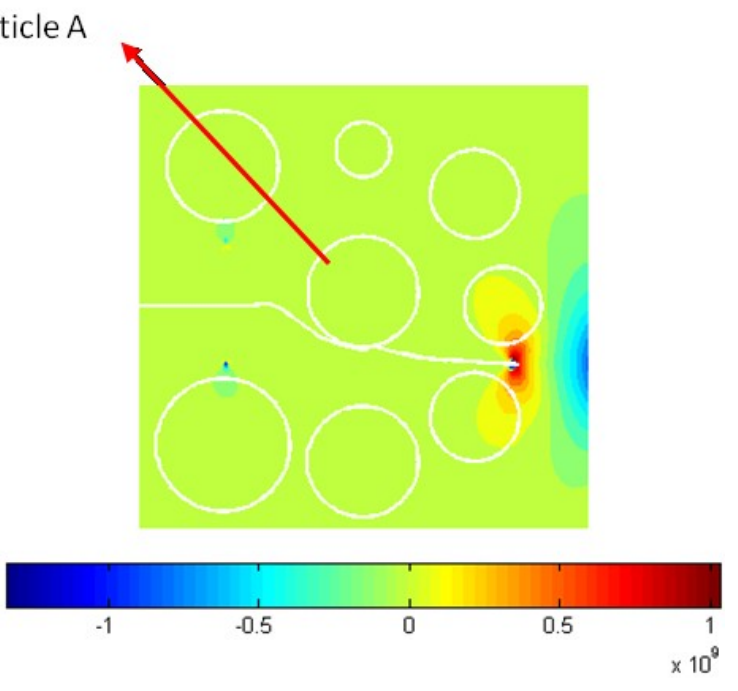

(d)

Figure 2-9: Compact tension fracture simulation of idealized samples with circular particles on two different locations ( $e=3 d / 4$ and $d / 4$ ) with respect to the crack line, (a) and (b) illustrate the mesh and $\sigma_{y y}$ stress contour with propagated crack line for particle A located at $e=3 d / 4$ respectively, (c) and (d) illustrate the mesh and $\sigma_{y y}$ stress contour with propagated crack line for particle A located at $e=d / 4$ respectively $(\mathrm{Ng}$ and Dai 2012) - see permission in Appendix C3. 


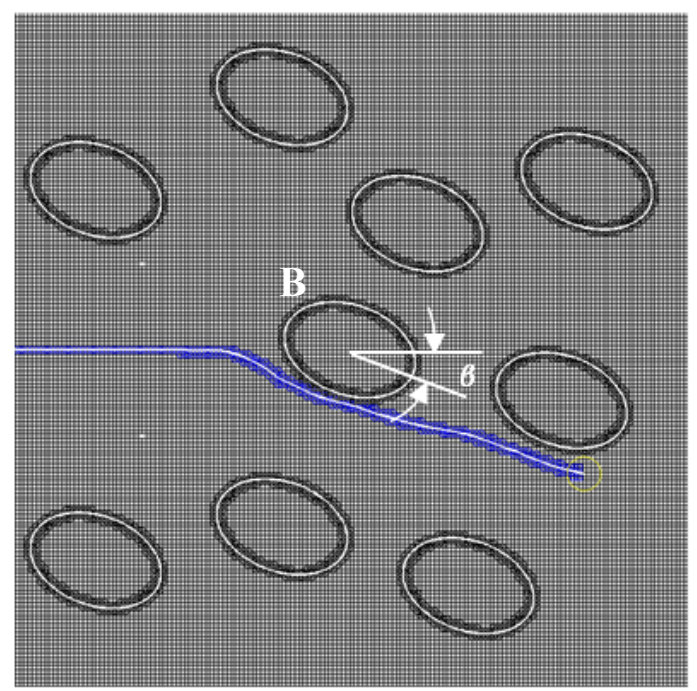

(a)

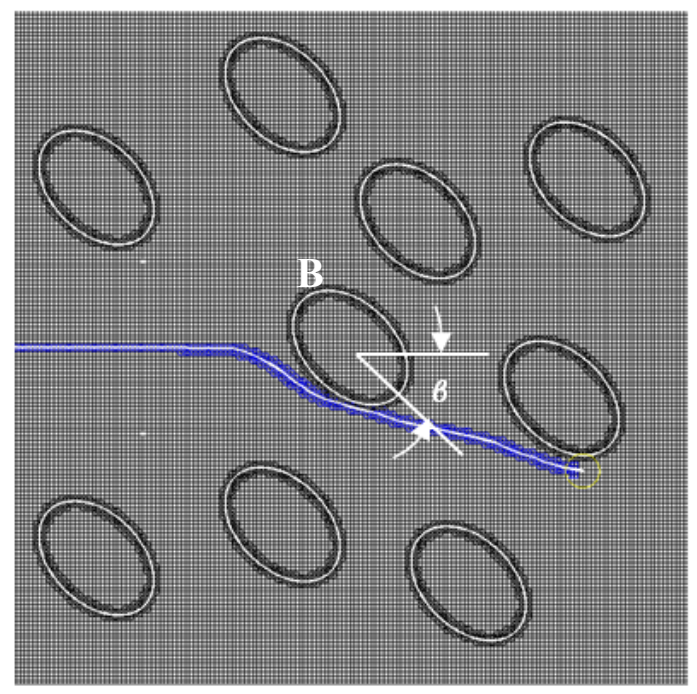

(c)

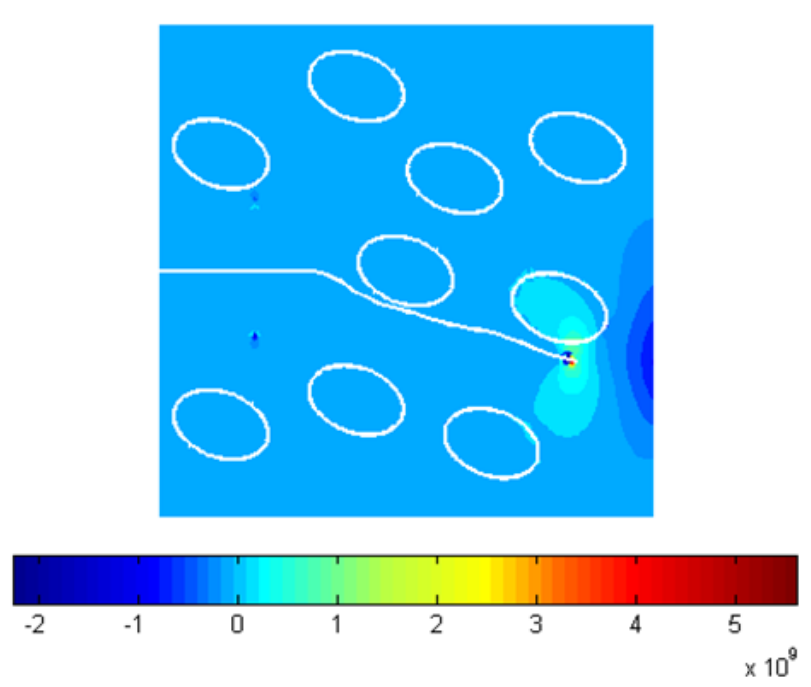

(b)

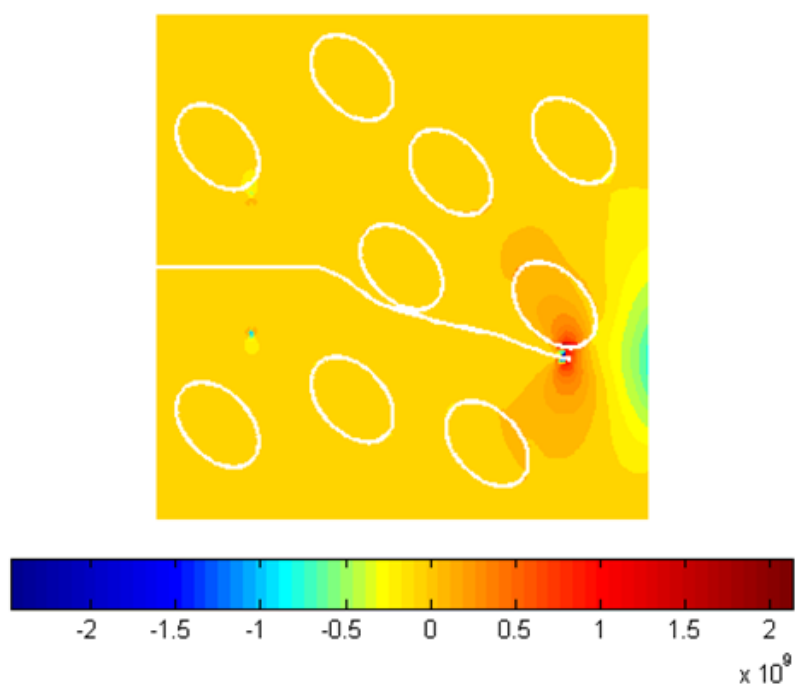

(d)

Figure 2-10: Compact tension fracture simulation of idealized samples consists of elliptical particles with shape factor of 1.5 at two orientations $\left(\beta=-20^{\circ}\right.$ and $\left.-45^{\circ}\right)$. (a) and (b) illustrate the mesh and $\sigma_{y y}$ stress contour with propagated crack line for particle oriented at $\beta=-20^{\circ}$, respectively. (c) and (d) illustrate the mesh and $\sigma_{y y}$ stress contour with propagated crack line for particle oriented at $\beta=-45^{\circ}$, respectively ( $\mathrm{Ng}$ and Dai 2012) - see permission in Appendix C3. 


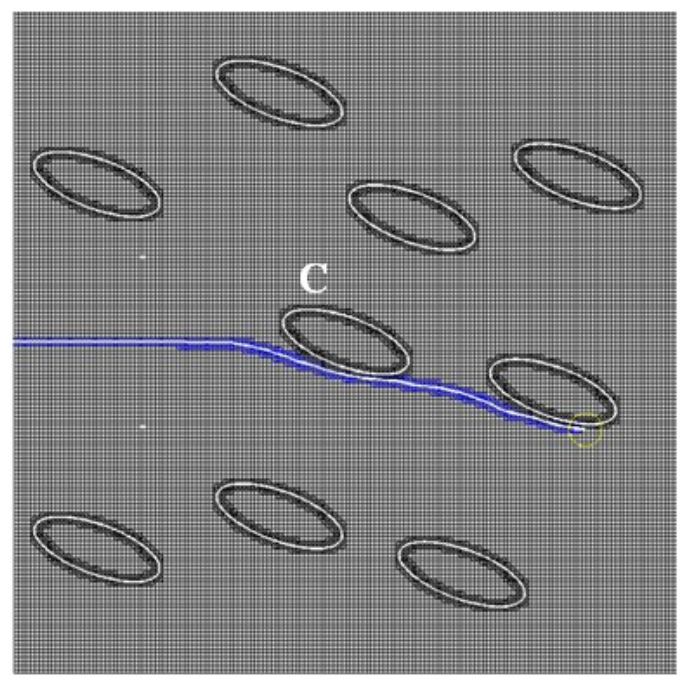

(a)

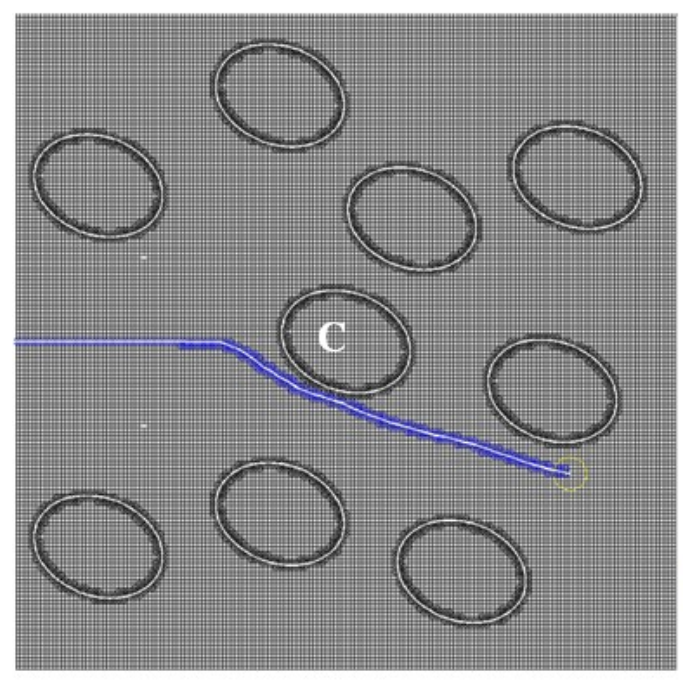

(c)

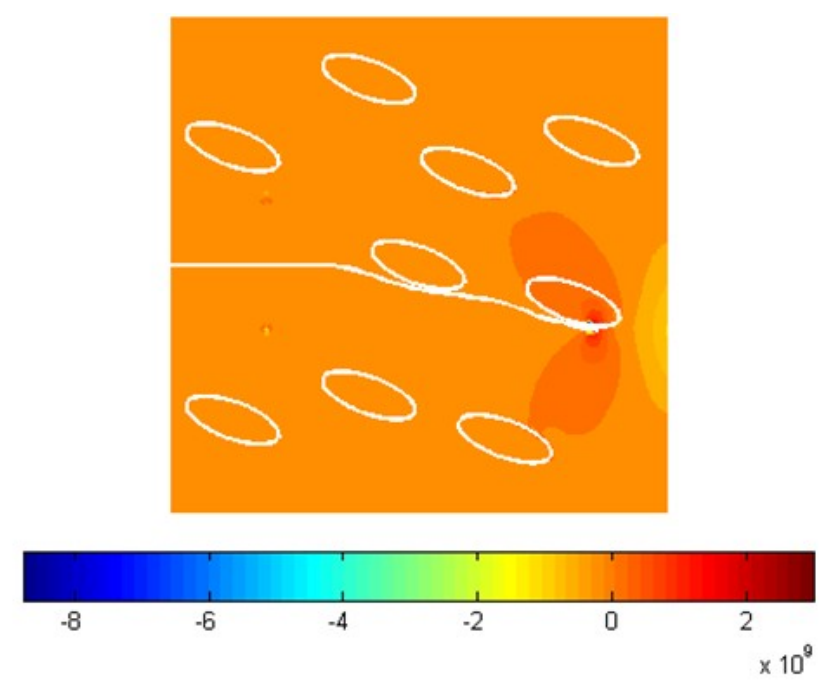

(b)

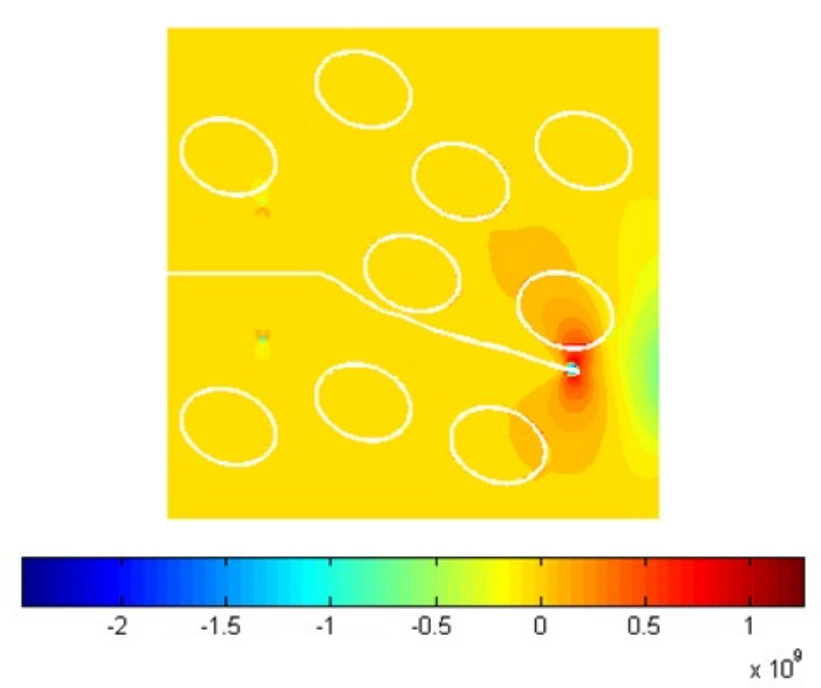

(d)

Figure 2-11: Compact tension fracture simulation of idealized samples that are consisted of elliptical particles oriented at $\alpha=-20^{\circ}$ with two shape factors $(S F=2.67$ and 1.33), (a) and (b) illustrate the mesh and $\sigma_{y y}$ stress contour with propagated crack line for particles $S F=2.67$ respectively, (c) and (d) illustrate the mesh and $\sigma_{y y}$ stress contour with propagated crack line for particle $S F=1.33$ respectively (Ng and Dai 2012) - see permission in Appendix C3.

Overall, the crack propagation was affected by the particle micro-parameters including deployments, orientations and shape factors. The stress contours show the 
interactions between the cement and particles along the interfacial zones. These interactions will change the maximum $G$ and thus the propagation path based on Equation (2-14). This doctoral study indicates that the microstructure of cement-based materials can largely affect the crack propagation and deformation fields. Our future study will be extended to simulate the crack propagation within the real concrete microstructure by utilizing the X-ray computed tomography images.

\subsection{Bilinear Cohesive Zone Modeling Technique}

\subsubsection{Theoretical and numerical aspects}

The bilinear cohesive zone model (CZM) technique was utilized for this doctoral study rather than the potential based exponential cohesive law. This is because bilinear CZM technique solves the artificial compliance issue due to pre-peak slope in the potential based exponential cohesive law by introducing an adjustable pre-peak slope, which will be presented in the next section. In this section, 2-D and 3-D theoretical and numerical aspects will be presented.

\subsubsection{Theoretical 2-D Bilinear CZM}

For 2-D bilinear CZM, the non-dimensional effective displacement $\left(\lambda_{e}\right)$ and effective traction $\left(t_{e}\right)$ are given as follows:

$$
\begin{gathered}
\lambda_{e}=\sqrt{\left(\frac{\delta_{n}}{\delta_{c}}\right)^{2}+\left(\frac{\delta_{s}}{\delta_{c}}\right)^{2}} \\
t_{e}=\sqrt{t_{n}^{2}+t_{s}^{2}}
\end{gathered}
$$


where $\delta_{n}$ and $\delta_{s}$ are the normal displacement and shear sliding on crack surface, respectively; $\delta_{c}$ is the critical displacement that indicates a complete separation of fracture surface with zero traction; $t_{n}$ and $t_{s}$ are the normal and shear tractions, respectively.

The relations of normal and shear tractions of two stages are described as follows:

$$
\left.\begin{array}{l}
t_{n}=\sigma_{c} \frac{1}{\lambda_{c r}}\left(\frac{\delta_{n}}{\delta_{c}}\right) \\
t_{s}=\sigma_{c} \frac{1}{\lambda_{c r}}\left(\frac{\delta_{s}}{\delta_{c}}\right)
\end{array}\right\} \text { for } \lambda_{e}<\lambda_{c r}
$$

and

$$
\left.\begin{array}{l}
t_{s}=\sigma_{c} \frac{1-\lambda_{e}}{1-\lambda_{c r}} \frac{1}{\lambda_{e}}\left(\frac{\delta_{s}}{\delta_{c}}\right) \\
t_{n}=\sigma_{c} \frac{1-\lambda_{e}}{1-\lambda_{c r}} \frac{1}{\lambda_{e}}\left(\frac{\delta_{n}}{\delta_{c}}\right)
\end{array}\right\} \text { for } \lambda_{e}>\lambda_{c r}
$$

where $\lambda_{c r}$ denotes non-dimensional critical displacement which occurs when the traction reaches maximum. Meanwhile, the tangent modulus matrix $(\mathbf{C})$, is a result of differentiation of traction with respect to the relative displacements, as follows:

$$
\mathbf{C}=\left[\begin{array}{cc}
C_{n n} & C_{n s} \\
-C_{s n} & C_{s s}
\end{array}\right]=\left[\begin{array}{ll}
\frac{\partial t_{n}}{\partial \delta_{n}} & \frac{\partial t_{n}}{\partial \delta_{s}} \\
\frac{\partial t_{s}}{\partial \delta_{n}} & \frac{\partial t_{s}}{\partial \delta_{s}}
\end{array}\right]
$$

where the components of the tangent modulus matrix for two stages are as follows: 


$$
\left.\begin{array}{l}
C_{s s}=C_{n n}=\frac{\sigma_{c}}{\lambda_{c r} \delta_{c}} \\
C_{s n}=C_{n s}=0
\end{array}\right\} \quad \text { for } \lambda_{e}<\lambda_{c r}
$$

and

$$
\left.\begin{array}{l}
C_{s s}=-\frac{\delta_{c} \sigma_{c}}{1-\lambda_{c r}}\left(\frac{\delta_{s}}{\lambda_{e} \delta_{c}^{2}}\right)^{2}+\left(1-\lambda_{e}\right) \frac{\delta_{c} \sigma_{c}}{1-\lambda_{c r}}\left(\frac{1}{\lambda_{e} \delta_{c}^{2}}-\frac{1}{\lambda_{e}^{3}} \frac{\delta_{s}^{2}}{\delta_{c}^{4}}\right) \\
C_{s n}=C_{n s}=-\frac{\delta_{c} \sigma_{c}}{1-\lambda_{c r}}\left(\frac{\delta_{s}}{\lambda_{e} \delta_{c}^{2}}\right)\left(\frac{\delta_{n}}{\lambda_{e} \delta_{c}^{2}}\right)+\left(1-\lambda_{e}\right) \frac{\delta_{c} \sigma_{c}}{1-\lambda_{c r}}\left(-\frac{1}{\lambda_{e}^{3}} \frac{\delta_{s} \delta_{n}}{\delta_{c}^{4}}\right) \\
C_{n n}=-\frac{\delta_{c} \sigma_{c}}{1-\lambda_{c r}}\left(\frac{\delta_{n}}{\lambda_{e} \delta_{c}^{2}}\right)^{2}+\left(1-\lambda_{e}\right) \frac{\delta_{c} \sigma_{c}}{1-\lambda_{c r}}\left(\frac{1}{\lambda_{e} \delta_{c}^{2}}-\frac{1}{\lambda_{e}^{3}} \frac{\delta_{n}^{2}}{\delta_{c}^{4}}\right)
\end{array}\right\} \text { for } \lambda_{e}>\lambda_{c r}
$$

As shown in Figure 2-12, the initial slope is the elastic part of the intrinsic cohesive law. Meanwhile, the decaying curve after the threshold point is the softening process when various damages formed in the fracture process zone. The adjustable prepeak slope solves the compliance issue in the potential based exponential cohesive law. 


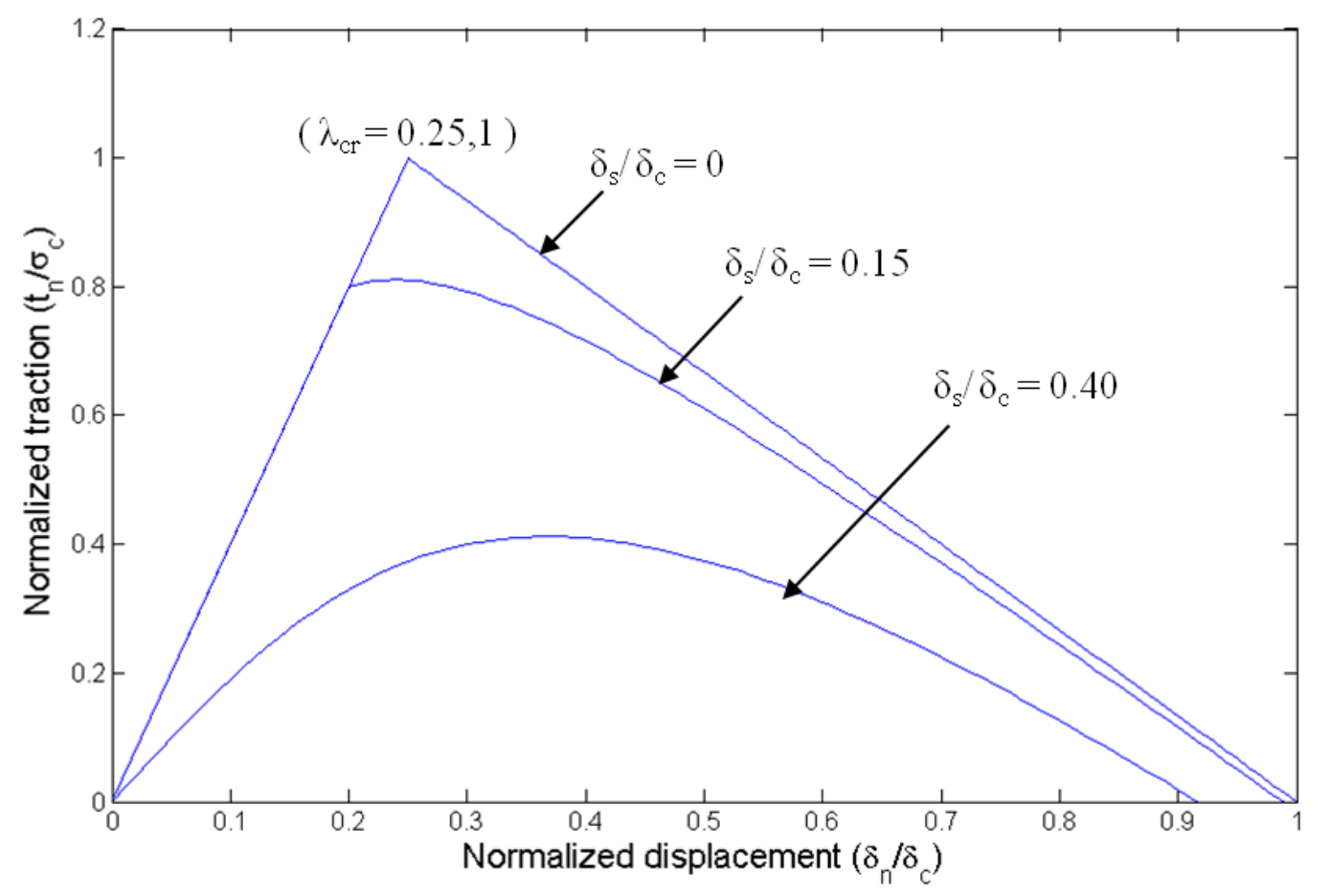

Figure 2-12: Schematic plot of bilinear cohesive zone model which demonstrate a linear relation before reaching the peak load and decays to zero monotonically.

The cohesive fracture energy $\left(G_{c}\right)$ is the area under the normalized displacementtraction curve (Figure 2-12) which is also equivalent to:

$$
G_{c}=\frac{1}{2} \delta_{c} \sigma_{c}
$$

\subsubsection{2-D cohesive element formulation}

Figure 2-13 demonstrates the cohesive element in the CZM that is constructed by two truss elements with a total of four nodes. The ordering of the node number is in the counter-clockwise direction: with Nodes 1 and 2 are located in the truss element at the bottom while Nodes 3 and 4 are located in the truss element at the top. The $\mathrm{X}-\mathrm{Y}$ coordinate system refers to the global coordinates while n-s coordinate system refers to 
the local coordinates. The force vector and the tangent stiffness matrix are defined in the local coordinate with the cohesive law. The global matrices can be obtained through the transformation matrix with an angle, $\theta$, as shown in the equation below:

$$
\mathbf{T}=\left[\begin{array}{cc}
\cos \theta & \sin \theta \\
-\sin \theta & \cos \theta
\end{array}\right]
$$

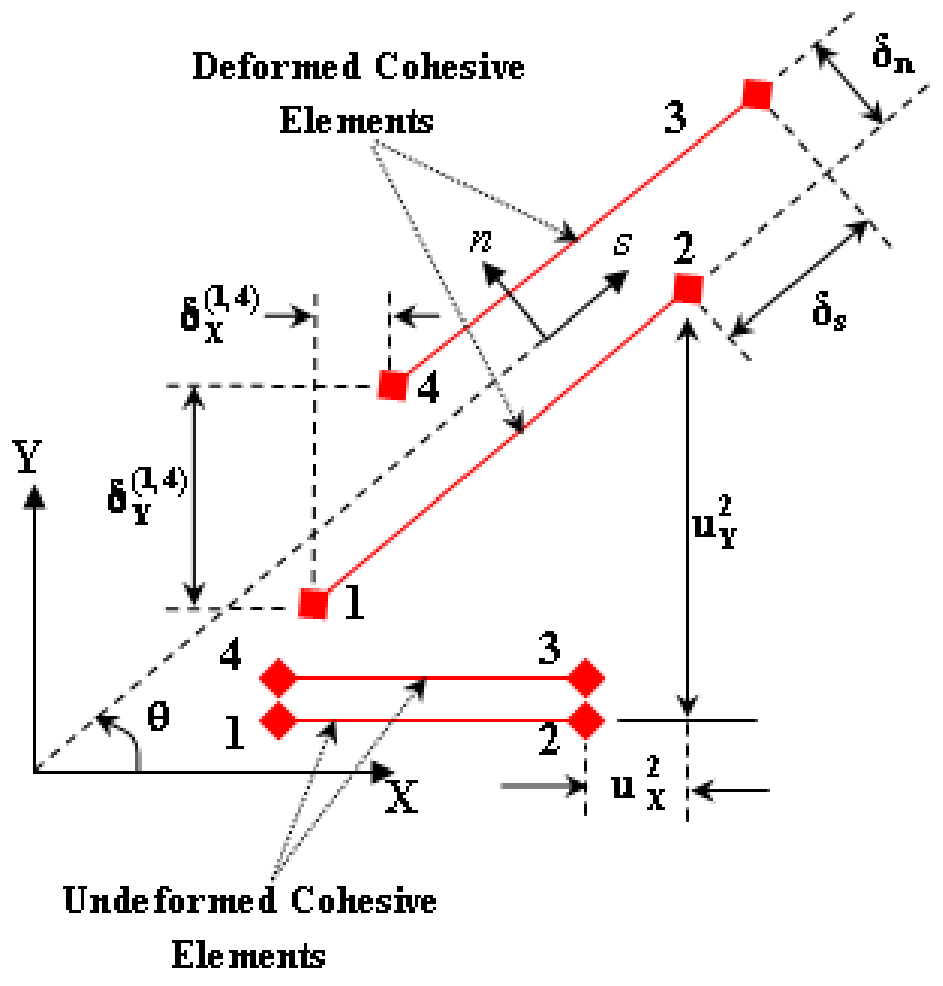

Figure 2-13: Schematic of a cohesive zone element with four nodes in the global (X-Y) coordinates for undeformed conditions and local coordinates for deformed case, with an orientation angle $\theta$.

With two degrees of freedom at each node, the global displacement vector of the cohesive elements is given as:

$$
\mathbf{U}=\left[\begin{array}{llllllll}
u_{1} & v_{1} & u_{2} & v_{2} & u_{3} & v_{3} & u_{4} & v_{4}
\end{array}\right]^{T}
$$


where $u$ and $v$ denote the global displacement in $\mathrm{X}$ and $\mathrm{Y}$ directions, respectively. The subscripts denote the node numbers while $\boldsymbol{T}$ refers to the transpose of matrix. The global relative displacements between the Nodes 1 and 2 and Nodes 3 and 4 are given as follows:

$$
\left\{\begin{array}{l}
\delta_{u}^{(1,4)} \\
\delta_{v}^{(1,4)} \\
\delta_{u}^{(2,3)} \\
\delta_{v}^{(2,3)}
\end{array}\right\}=\mathbf{L} \mathbf{U}, \mathbf{L}=\left[\begin{array}{cccccccc}
1 & 0 & 0 & 0 & 0 & 0 & -1 & 0 \\
0 & 1 & 0 & 0 & 0 & 0 & 0 & -1 \\
0 & 0 & 1 & 0 & -1 & 0 & 0 & 0 \\
0 & 0 & 0 & 1 & 0 & -1 & 0 & 0
\end{array}\right]
$$

where $\mathbf{L}$ is an operator matrix,. The superscripts represent the node pair with the relative displacement. The relative global displacement $\left(\delta_{X}\right.$ and $\left.\delta_{Y}\right)$ functions are given as:

$$
\left\{\begin{array}{l}
\delta_{X} \\
\delta_{Y}
\end{array}\right\}=\left[\begin{array}{cccc}
N_{1} & 0 & N_{2} & 0 \\
0 & N_{1} & 0 & N_{2}
\end{array}\right]\left\{\begin{array}{l}
\delta_{u}^{(1,4)} \\
\delta_{v}^{(1,4)} \\
\delta_{u}^{(2,3)} \\
\delta_{v}^{(2,3)}
\end{array}\right\}=\mathbf{N}\left\{\begin{array}{l}
\delta_{u}^{(1,4)} \\
\delta_{v}^{(1,4)} \\
\delta_{u}^{(2,3)} \\
\delta_{v}^{(2,3)}
\end{array}\right\}=\mathbf{N L U}
$$

where $N$ denotes the shape function with iso-parametric coordinate, $\xi$. Hence, the relative local displacement vector $\left(\delta_{s}\right.$ and $\left.\delta_{n}\right)$ can be obtained by multiplying the transformation matrix $[T]$ (Equation (2-32)) with the relative global displacements (Equation (2-34)) as followings:

$$
\left\{\begin{array}{l}
\delta_{s} \\
\delta_{n}
\end{array}\right\}=\mathbf{B U}=\mathbf{T N L U}
$$

Finally, the global nodal force vector, $\mathbf{f}$ and global tangent stiffness matrix, $\mathbf{k}$ of the cohesive element are given as follows: 


$$
\begin{gathered}
\mathbf{f}=\int_{-1}^{1} \mathbf{B}^{T} \mathbf{t}|\mathbf{J}| d \eta \\
\mathbf{k}=\int_{-1}^{1} \mathbf{B}^{T} \mathbf{C} \mathbf{B}|\mathbf{J}| d \eta
\end{gathered}
$$

where $\mathbf{J}$ represent the Jacobian matrix between reference and original coordinates, and $\mathbf{C}$ is the tangent modulus matrix. $t$ is the traction vector that are evaluated from Equation $(2-25)$.

\subsubsection{Theoretical 3-D Bilinear CZM}

For 3-D cohesive element, the effective displacement $\left(\lambda_{e}\right)$ and effective traction $\left(t_{e}\right)$ are as follows:

$$
\begin{gathered}
\lambda_{e}=\sqrt{\left(\frac{\delta_{n}}{\delta_{c}}\right)^{2}+\left(\frac{\delta_{s 1}}{\delta_{c}}\right)^{2}+\left(\frac{\delta_{s 2}}{\delta_{c}}\right)^{2}} \\
t_{e}=\sqrt{t_{n}^{2}+t_{s 1}^{2}+t_{s 2}^{2}}
\end{gathered}
$$

where the subscripts $s 1$ and $s 2$ denote the components of shearing directions in 3-D. The relations of normal and shear tractions of two stages for 3-D analysis are given as follows:

$$
t_{n}=\sigma_{c} \frac{1}{\lambda_{c r}}\left(\frac{\delta_{n}}{\delta_{c}}\right) ; t_{s 1}=\sigma_{c} \frac{1}{\lambda_{c r}}\left(\frac{\delta_{s 1}}{\delta_{c}}\right) ; t_{s 2}=\sigma_{c} \frac{1}{\lambda_{c r}}\left(\frac{\delta_{s 2}}{\delta_{c}}\right) \quad \text { for } \quad \lambda_{e}<\lambda_{c r}
$$




$$
\left.\begin{array}{rl}
t_{n} & =\sigma_{c} \frac{1-\lambda_{e}}{1-\lambda_{c r}} \frac{1}{\lambda_{e}}\left(\frac{\delta_{n}}{\delta_{c}}\right) \\
t_{s 1} & =\sigma_{c} \frac{1-\lambda_{e}}{1-\lambda_{c r}} \frac{1}{\lambda_{e}}\left(\frac{\delta_{s 1}}{\delta_{c}}\right) \\
t_{s 2} & =\sigma_{c} \frac{1-\lambda_{e}}{1-\lambda_{c r}} \frac{1}{\lambda_{e}}\left(\frac{\delta_{s 2}}{\delta_{c}}\right)
\end{array}\right\} \text { for } \lambda_{e}>\lambda_{c r}
$$

The tangent modulus matrix, $\mathbf{C}$, for 3-D model is described as follows:

$$
\mathbf{C}=\left[\begin{array}{ccc}
C_{n n} & C_{n s 1} & C_{n s 2} \\
-C_{s 1 n} & C_{s 1 s 1} & C_{s 1 s 2} \\
-C_{s 2 n} & -C_{s 2 s 1} & C_{s 2 s 2}
\end{array}\right]=\left[\begin{array}{lll}
\frac{\partial t_{n}}{\partial \delta_{n}} & \frac{\partial t_{n}}{\partial \delta_{s 1}} & \frac{\partial t_{n}}{\partial \delta_{s 2}} \\
\frac{\partial t_{s 1}}{\partial \delta_{n}} & \frac{\partial t_{s 1}}{\partial \delta_{s 1}} & \frac{\partial t_{s 1}}{\partial \delta_{s 2}} \\
\frac{\partial t_{s 2}}{\partial \delta_{n}} & \frac{\partial t_{s 2}}{\partial \delta_{s 1}} & \frac{\partial t_{s 2}}{\partial \delta_{s 2}}
\end{array}\right]
$$

The conditions of the tangent modulus matrix in two stages are described as:

$$
\left.\begin{array}{l}
C_{s 1 s 1}=C_{s 2 s 2}=C_{n n}=\frac{\sigma_{c}}{\lambda_{c r} \delta_{c}} \\
C_{s 1 n}=C_{s 2 n}=C_{s 2 s 1}=C_{s 1 s 2}=C_{n s 1}=C_{n s 2}=0
\end{array}\right\} \quad \text { for } \lambda_{e}<\lambda_{c r}
$$

and 


$$
\left.\begin{array}{l}
C_{n n}=\frac{\delta_{c} \sigma_{c}}{1-\lambda_{c r}}\left[\left(1-\lambda_{e}\right)\left(\frac{1}{\lambda_{e} \delta_{c}^{2}}-\frac{1}{\lambda_{e}^{3}} \frac{\delta_{n}^{2}}{\delta_{c}^{4}}\right)-\left(\frac{\delta_{n}}{\lambda_{e} \delta_{c}^{2}}\right)^{2}\right] \\
C_{s 1 s 1}=\frac{\delta_{c} \sigma_{c}}{1-\lambda_{c r}}\left[\left(1-\lambda_{e}\right)\left(\frac{1}{\lambda_{e} \delta_{c}^{2}}-\frac{1}{\lambda_{e}^{3}} \frac{\delta_{s 1}^{2}}{\delta_{c}^{4}}\right)-\left(\frac{\delta_{s 1}}{\lambda_{e} \delta_{c}^{2}}\right)^{2}\right] \\
C_{s 2 s 2}=\frac{\delta_{c} \sigma_{c}}{1-\lambda_{c r}}\left[\left(1-\lambda_{e}\right)\left(\frac{1}{\lambda_{e} \delta_{c}^{2}}-\frac{1}{\lambda_{e}^{3}} \frac{\delta_{s 2}^{2}}{\delta_{c}^{4}}\right)-\left(\frac{\delta_{s 2}}{\lambda_{e} \delta_{c}^{2}}\right)^{2}\right] \\
C_{s 1 n}=C_{n s 1}=\frac{\sigma_{c}}{1-\lambda_{c r}}\left[\left(1-\lambda_{e}\right)\left(-\frac{1}{\lambda_{e}^{3}} \frac{\delta_{s 1} \delta_{n}}{\delta_{c}^{3}}\right)-\left(\frac{\delta_{s 1} \delta_{n}}{\lambda_{e}^{2} \delta_{c}^{3}}\right)\right] \\
C_{s 2 n}=C_{n s 2}=\frac{\sigma_{c}}{1-\lambda_{c r}}\left[\left(1-\lambda_{e}\right)\left(-\frac{1}{\lambda_{e}^{3}} \frac{\delta_{s 2} \delta_{n}}{\delta_{c}^{3}}\right)-\left(\frac{\delta_{s 2} \delta_{n}}{\lambda_{e}^{2} \delta_{c}^{3}}\right)\right] \\
C_{s 1 s 2}=C_{s 2 s 1}=\frac{\sigma_{c}}{1-\lambda_{c r}}\left[\left(1-\lambda_{e}\right)\left(-\frac{1}{\lambda_{e}^{3}} \frac{\delta_{s 1} \delta_{s 2}}{\delta_{c}^{3}}\right)-\left(\frac{\delta_{s 1} \delta_{s 2}}{\lambda_{e}^{2} \delta_{c}^{3}}\right)\right]
\end{array}\right\}
$$

Similar to 2-D case, the fracture energy is equivalent to Equation (2-31).

\subsubsection{3-D cohesive element formulation}

In the 3-D cohesive element model, the cohesive element is 8-noded, as shown in

Figure 2-14. That is, one side of the cohesive element consists of Nodes 1, 2, 3, and 4 (Side 1), whereas another side consists of Nodes 5, 6, 7, and 8 (Side 2). Thus, with 3 degrees of freedom at each node, the global displacement vector of the 8-noded cohesive element is given as:

$$
\mathbf{U}=\left[\begin{array}{lllllllllllll}
u_{1} & v_{1} & w_{1} & u_{2} & v_{2} & w_{2} & \ldots & u_{7} & v_{7} & w_{7} & u_{8} & v_{8} & w_{8}
\end{array}\right]^{T}
$$

where $u, v$, and $w$ denote the global displacement in $\mathrm{X}, \mathrm{Y}$, and $\mathrm{Z}$ directions, respectively. The subscripts denote the node numbers while $\boldsymbol{T}$ refers to the transpose of matrix. 


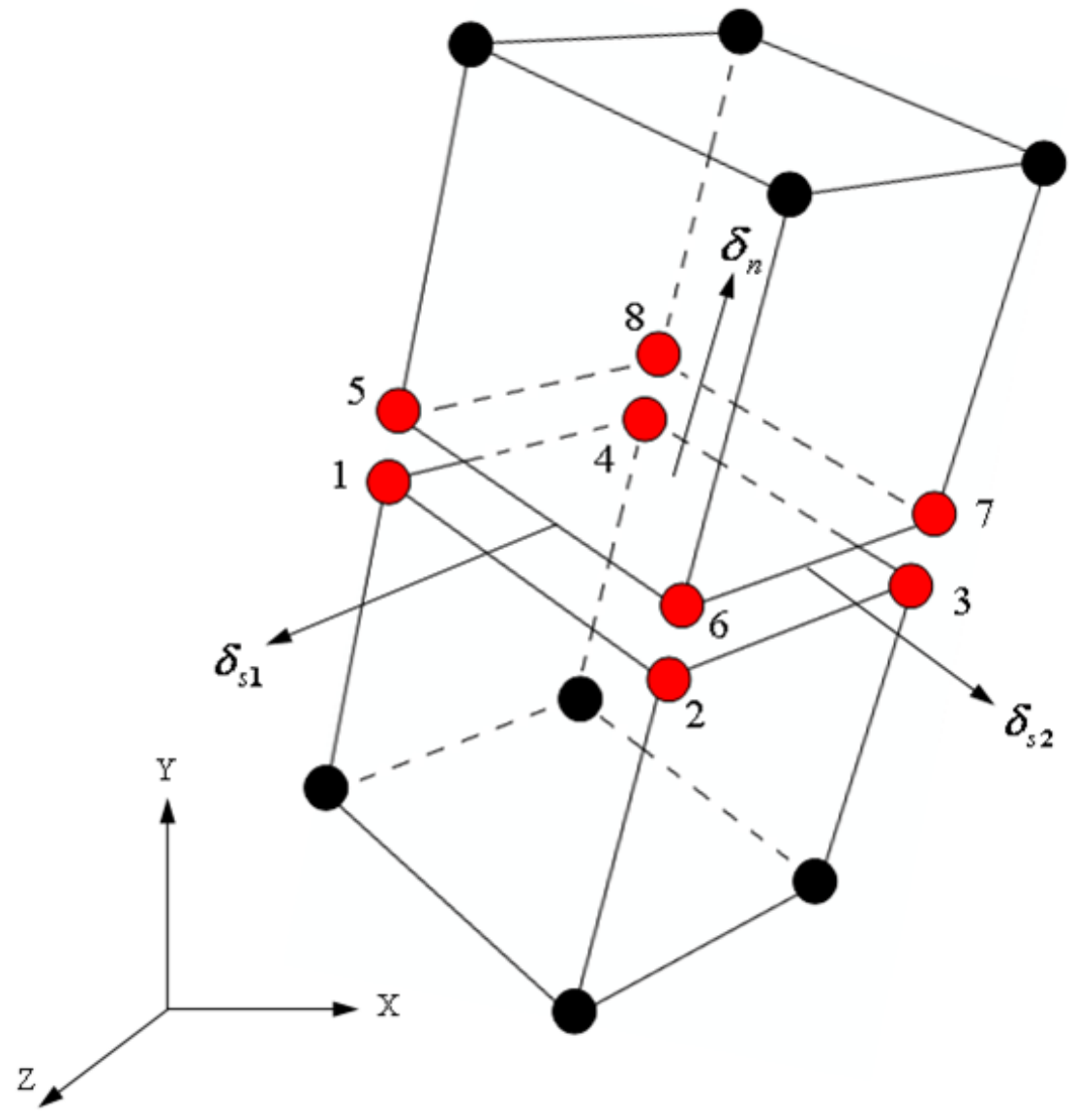

Figure 2-14: Schematic 3-D cohesive zone element that is built up with 8 nodes (red spheres) in the global (X,Y, and Z) coordinates and local (n, s1, and s2) coordinates. With Side 1 consists of nodes 1, 2, 3, and 4, whereas Side 2 consists of nodes 5, 6, 7, and 8 .

The global relative displacements between the nodes on Side 1 and Side 2 are given as follows: 


$$
\left\{\begin{array}{l}
\delta_{u}^{(1,5)} \\
\delta_{v}^{(1,5)} \\
\delta_{w}^{(1,5)} \\
\delta_{u}^{(2,6)} \\
\delta_{v}^{(2,6)} \\
\delta_{w}^{(2,6)} \\
\delta_{u}^{(3,7)} \\
\delta_{v}^{(3,7)} \\
\delta_{w}^{(3,7)} \\
\delta_{u}^{(4,8)} \\
\delta_{v}^{(4,8)} \\
\delta_{w}^{(4,8)}
\end{array}\right\}=\mathbf{L} \mathbf{U},
$$

$\mathbf{L}=\left[\begin{array}{cccccccccccccccccccccccc}1 & 0 & 0 & 0 & 0 & 0 & 0 & 0 & 0 & 0 & 0 & 0 & -1 & 0 & 0 & 0 & 0 & 0 & 0 & 0 & 0 & 0 & 0 & 0 \\ 0 & 1 & 0 & 0 & 0 & 0 & 0 & 0 & 0 & 0 & 0 & 0 & 0 & -1 & 0 & 0 & 0 & 0 & 0 & 0 & 0 & 0 & 0 & 0 \\ 0 & 0 & 1 & 0 & 0 & 0 & 0 & 0 & 0 & 0 & 0 & 0 & 0 & 0 & -1 & 0 & 0 & 0 & 0 & 0 & 0 & 0 & 0 & 0 \\ 0 & 0 & 0 & 1 & 0 & 0 & 0 & 0 & 0 & 0 & 0 & 0 & 0 & 0 & 0 & -1 & 0 & 0 & 0 & 0 & 0 & 0 & 0 & 0 \\ 0 & 0 & 0 & 0 & 1 & 0 & 0 & 0 & 0 & 0 & 0 & 0 & 0 & 0 & 0 & 0 & -1 & 0 & 0 & 0 & 0 & 0 & 0 & 0 \\ 0 & 0 & 0 & 0 & 0 & 1 & 0 & 0 & 0 & 0 & 0 & 0 & 0 & 0 & 0 & 0 & 0 & -1 & 0 & 0 & 0 & 0 & 0 & 0 \\ \vdots & \vdots & \vdots & \vdots & \vdots & \vdots & \vdots & \vdots & \vdots & \vdots & \vdots & \vdots & \vdots & \vdots & \vdots & \vdots & \vdots & \vdots & \vdots & \vdots & \vdots & \vdots & \vdots & \vdots \\ 0 & 0 & 0 & 0 & 0 & 0 & 0 & 0 & 0 & 1 & 0 & 0 & 0 & 0 & 0 & 0 & 0 & 0 & 0 & 0 & 0 & -1 & 0 & 0 \\ 0 & 0 & 0 & 0 & 0 & 0 & 0 & 0 & 0 & 0 & 1 & 0 & 0 & 0 & 0 & 0 & 0 & 0 & 0 & 0 & 0 & 0 & -1 & 0 \\ 0 & 0 & 0 & 0 & 0 & 0 & 0 & 0 & 0 & 0 & 0 & 1 & 0 & 0 & 0 & 0 & 0 & 0 & 0 & 0 & 0 & 0 & 0 & -1\end{array}\right]$

where $\mathrm{L}$ is an operator matrix,. The superscripts represent the node pair with the relative displacement. The relative global displacement $\left(\delta_{X}, \delta_{Y}\right.$, and $\left.\delta_{Z}\right)$ functions are given as: 


$$
\left\{\begin{array}{l}
\delta_{X} \\
\delta_{Y} \\
\delta_{Z}
\end{array}\right\}=\left[\begin{array}{cccccccccccc}
N_{1} & 0 & 0 & N_{2} & 0 & 0 & N_{3} & 0 & 0 & N_{4} & 0 & 0 \\
0 & N_{1} & 0 & 0 & N_{2} & 0 & 0 & N_{3} & 0 & 0 & N_{4} & 0 \\
0 & 0 & N_{1} & 0 & 0 & N_{2} & 0 & 0 & N_{3} & 0 & 0 & N_{4}
\end{array}\right]\left\{\begin{array}{l}
\delta_{u}^{(1,5)} \\
\delta_{v}^{(1,5)} \\
\delta_{w}^{(1,5)} \\
\delta_{u}^{(2,6)} \\
\delta_{v}^{(2,6)} \\
\delta_{w}^{(2,6)} \\
\delta_{u}^{(3,7)} \\
\delta_{v}^{(3,7)} \\
\delta_{w}^{(3,7)} \\
\delta_{u}^{(4,8)} \\
\delta_{v}^{(4,8)} \\
\delta_{w}^{(4,8)}
\end{array}\right\}=\mathbf{N L \mathbf { U }}
$$

where, $N$ denotes the shape function with iso-parametric coordinate, as described below:

$$
N_{1}=\frac{(1-s)(1-t)}{4} ; \quad N_{2}=\frac{(1+s)(1-t)}{4} ; \quad N_{3}=\frac{(1+s)(1+t)}{4} ; \quad N_{4}=\frac{(1-s)(1+t)}{4}
$$

A tedious formulation for the transformation matrix, $T$ is developed which involves Jacobian matrix, $J$ and rotational matrix, $R$. The rotational matrix is developed based on the center point of the mid-plane between Side 1 and Side 2. The Jacobian matrix for 3D cohesive element is given as:

$$
J=\left[\begin{array}{lll}
\frac{\partial x}{\partial s 1} & \frac{\partial y}{\partial s 1} & \frac{\partial z}{\partial s 1} \\
\frac{\partial x}{\partial s 2} & \frac{\partial y}{\partial s 2} & \frac{\partial z}{\partial s 2}
\end{array}\right]=\left[\begin{array}{lll}
J_{1,1} & J_{1,2} & J_{1,3} \\
J_{2,1} & J_{2,2} & J_{2,3}
\end{array}\right]
$$

the elements in the row of the Jacobian matrix are the vectors on the tangent plane, whereas the normal vector is obtained from the cross product. After some manipulations, the rotational matrix is given as: 


$$
\mathbf{R}=\left[\begin{array}{ccc} 
& & \\
J_{1,1} / A_{1} & J_{1,2} / A_{1} & J_{1,3} / A_{1} \\
J_{2,1} / A_{2} & J_{2,2} / A_{2} & J_{2,3} / A_{2} \\
\frac{J_{1,2} J_{2,3}-J_{1,3} J_{2,2}}{A_{1} A_{2}} & \frac{J_{1,3} J_{2,1}-J_{1,1} J_{2,3}}{A_{1} A_{2}} & \frac{J_{1,1} J_{2,2}-J_{1,2} J_{2,1}}{A_{1} A_{2}}
\end{array}\right]
$$

where,

$$
A_{1}=\sqrt{{J_{1,1}{ }^{2}+{J_{1,2}}{ }^{2}+{J_{1,3}}^{2}}^{2}} \text { and } A_{2}=\sqrt{{J_{2,1}{ }^{2}+{J_{2,2}}{ }^{2}+{J_{2,3}}^{2}}^{2}}
$$

The transformation matrix, $\mathrm{T}$ is given as:

$$
T=\left[\begin{array}{llll}
{[\mathbf{R}]} & & & \\
& {[\mathbf{R}]} & & \\
& & \ddots & \\
& & & [\mathbf{R}]\rfloor(24 \times 24)
\end{array}\right.
$$

Hence, the relative local displacement vector $\left(\delta_{s 1}, \delta_{s 2}\right.$, and $\left.\delta_{n}\right)$ can be obtained by multiplying the transformation matrix (Equation (2-53)) with the relative global displacements (Equation (2-48)) as followings:

$$
\left\{\begin{array}{l}
\delta_{s 1} \\
\delta_{s 2} \\
\delta_{n}
\end{array}\right\}=\mathbf{B U}=\mathbf{R N L U}
$$

Finally, the global nodal force vector, $\mathbf{f}$ and global tangent stiffness matrix, $\mathbf{k}$ of the cohesive element are similar to the 2-D cohesive element formulation, that is, Equation (2-37) and Equation (2-38), respectively. 


\subsubsection{Fracture simulation on uniform or idealized samples}

In this section, both 2-D and 3-D fracture simulations on uniform or idealized samples will be presented. Among the fracture models that that were developed are the CT test and SEB bending test. Figure 2-15 demonstrates a CT test model that was simulated with crack openings along the predefined cohesive zone element boundary. The model was constructed based on standard dimensions (Anderson 1995) with $\mathrm{W}=80$ $\mathrm{mm}$, which is the dimension of the center of the hole to the model's right edge. Two holes with a radius of 5 elements were created, with the top hole defined with the load while the other hole was defined with fixed support. For homogeneous cement paste material, the elastic modulus and Poisson's ratio were defined as $30 \mathrm{GPa}$ and 0.30 respectively. For the bilinear CZM parameters, the fracture energy and tensile strength were defined as $37.5 \mathrm{~J} / \mathrm{m}^{2}$ and $15 \mathrm{GPa}$, respectively. To avoid elements overlapping at the end of the model's right edge, three rows of continuous elements were constructed. 

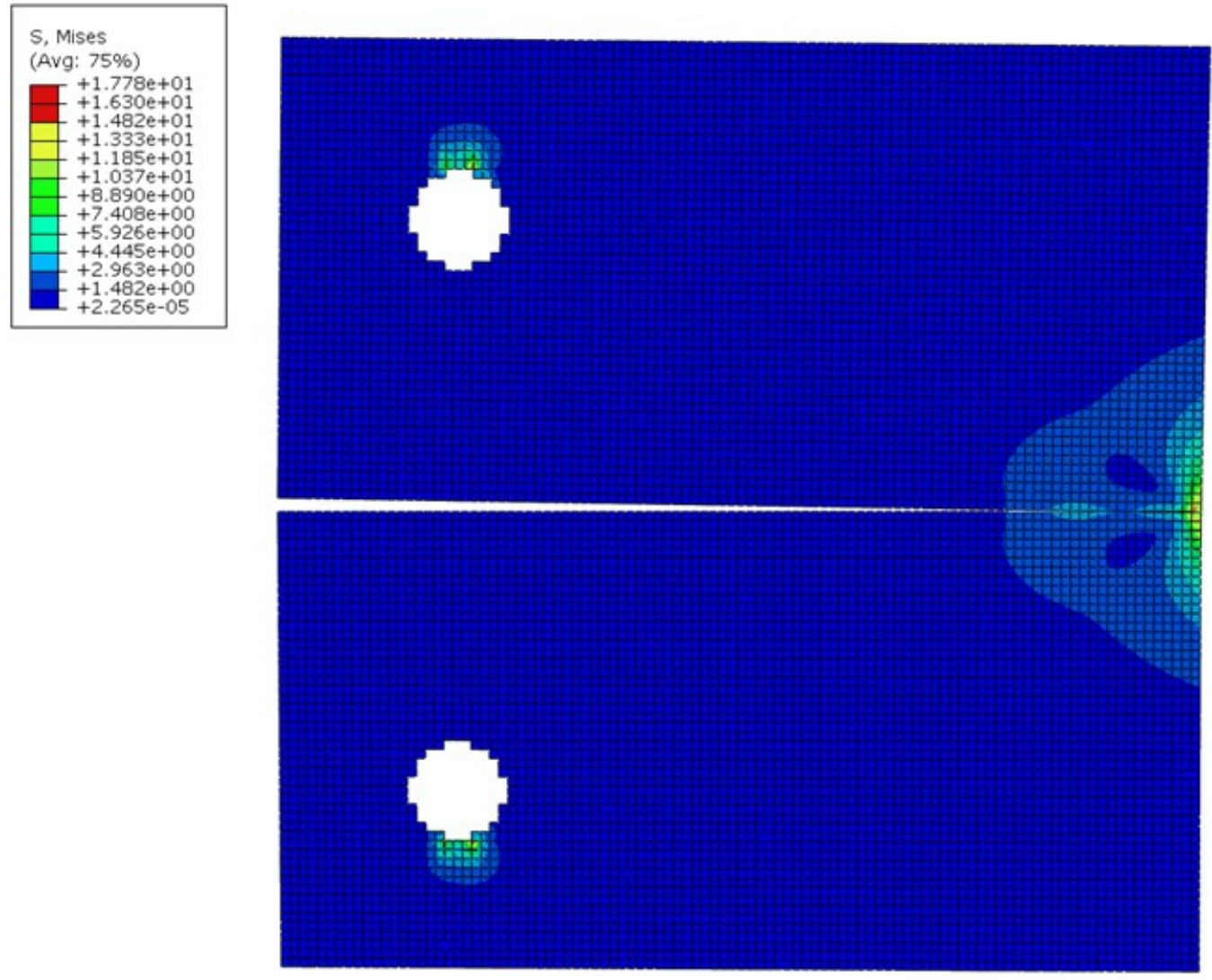

Figure 2-15: A 2-D fracture simulation of CT test on a homogeneous cement paste sample by using bilinear CZM technique.

3-D fracture simulations on homogeneous (Figure 2-16(a)) and two phase cement paste models (Figure 2-16) were also performed. Both models have the similar geometries and material properties as the 2-D model. In the two phase cement paste CT test model, it was shown that the bilinear CZM technique has the capability to simulate crack coalesces from one pore to another. The results show that bilinear CZM technique is feasible to simulate both homogeneous and pore system models. 

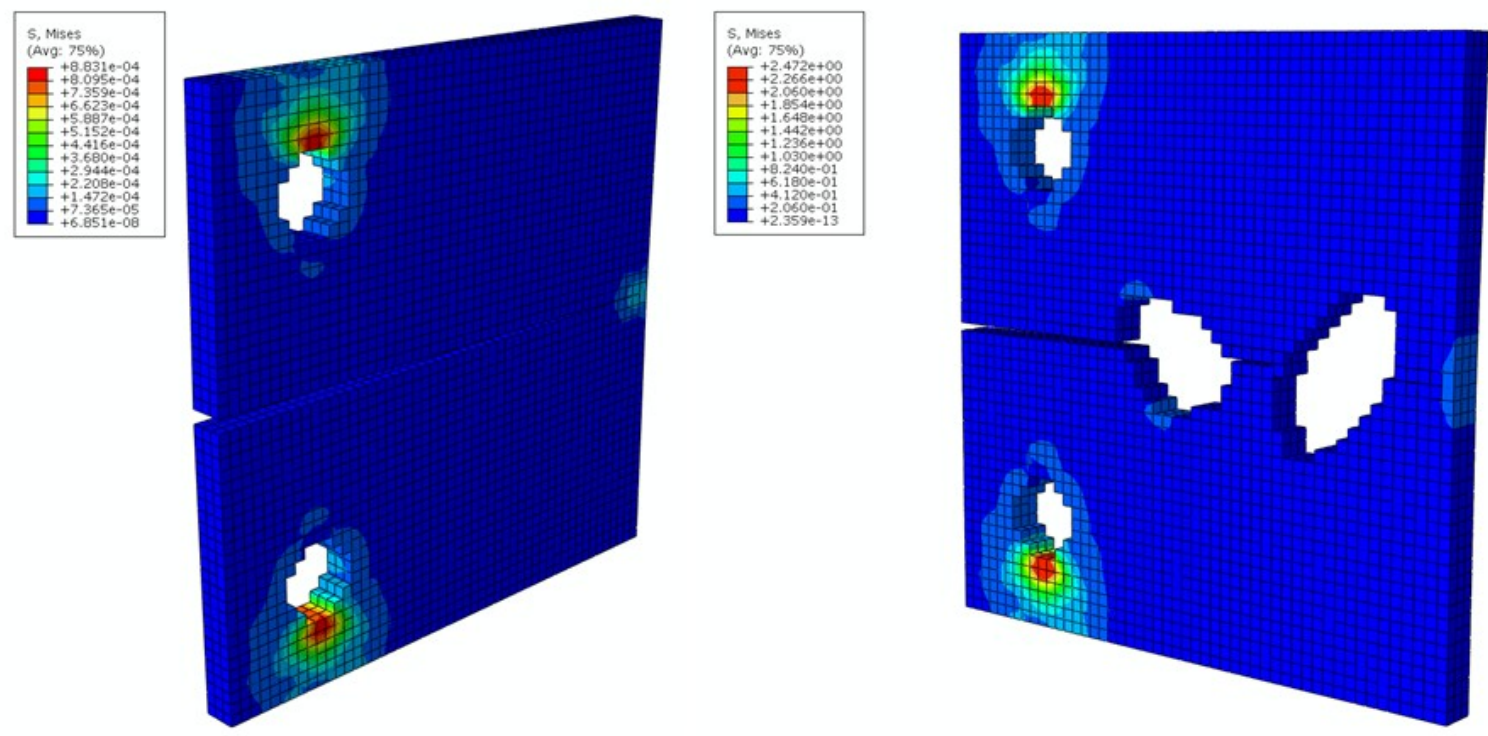

Figure 2-16: 3-D fracture simulation of CT test by using bilinear CZM technique. (a) A 3-D homogenous cement paste model. (b) A two phase cement paste model which shows the crack coalesces from one pore to another.

Figure 2-17 demonstrates a fracture simulation of the SEB test on a homogeneous concrete sample by using the bilinear CZM technique with the same material properties defined in the previous cases. The geometry dimensions were selected to meet the standard ASTM C 293-08 SEB bending test requirements, which is similar to the XFEM simulation case as demonstrated in Figure 2-7(a) (Section 2.2.2.2). That is, with a roller support and a fixed support applied on each side on the bottom face, and a unit displacement loading on the top surface of the model. The result shows that the fracture simulation on the SEB bending test is also feasible to be modeled. 

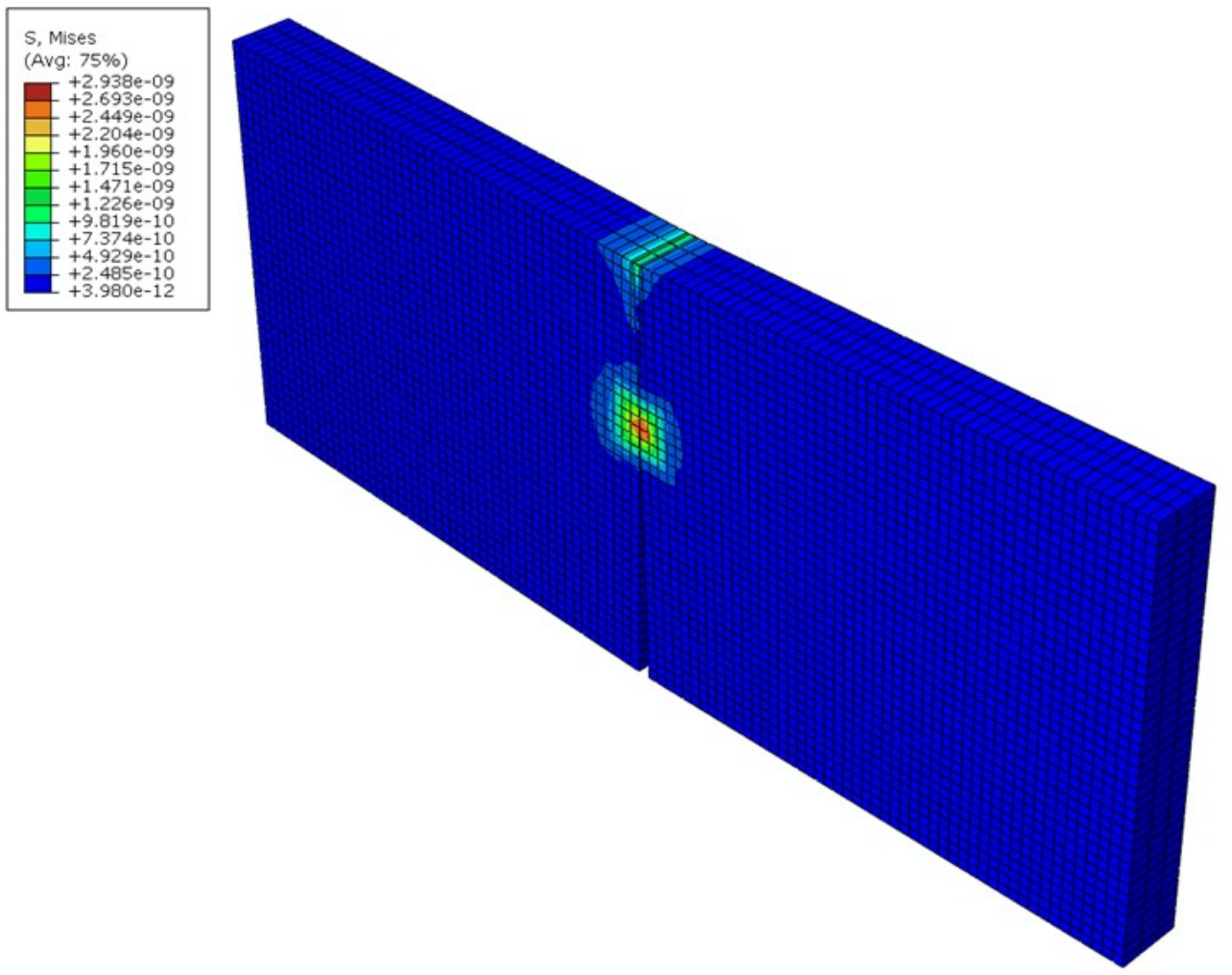

Figure 2-17: A 3-D fracture simulation of SEB test on a homogeneous cement paste sample by using bilinear CZM technique.

\subsection{Concluding Remarks}

There are many options to choose for performing fracture simulations. Through the thorough literature studies, the XFEM and the bilinear CZM techniques were selected as the fracture modeling techniques in this doctoral study to investigate the fracture behavior on cement-based samples. The XFEM program was executed in MATLAB whereas the bilinear CZM program was executed in the ABAQUS with user subroutines. Prior employing these techniques on analyzing the internal frost damage on cement-based sample, it is essential to validate the numerical simulation results to the analytical results. 
In this study, the compact tension (CT) test and single-edge beam (SEB) bending test were utilized for validation purposes.

The validation of XFEM simulation was done by comparing the computational and analytical stress intensity factors (SIFs) on the CT test and SEB bending test. All the models were developed in accordance to standard dimensions, such as ASTM standard. The homogeneous model simulations were firstly performed on the CT test and SEB bending test models. Both models showed well agreement results in the SIFs values determined computationally and analytically. The mixed-mode SEB bending test model also showed the capability of XFEM technique to divert the crack growth direction

In order to deal with heterogeneous media, such as concrete, the XFEM program was altered by introducing the search distance and kink angle schemes. The alteration was to improve the crack growth prediction result by preventing the crack cut through the inclusions. The approach had successfully modeled the crack growth in the idealized inclusions with various shapes, sizes, and orientations without penetrating into the inclusions on the CT test model. The breaktrough of the schemes allowed the fracture simulations can be performed on heterogeneous models, such as cement-based samples, by using the XFEM technique. The only drawbacks of the current developed XFEM program are: (1) unable to simulate crack growth in a multi-pore system and, (2) only 2D case can be performed.

On the other hand, the bilinear CZM technique was employed to overcome the limitations of the developed XFEM program. The 2-D fracture simulation of CT test of homogeneous sample was performed and showed that the crack openings as a result of a unit displacement loading applied. The simulation was extended to 3-D models with 
developed CT test and SEB bending test. The simulations were successful with crack openings developed, including the CT test model with pores. The results indicated that the bilinear CZM technique can be employed to simulate fracture simulation in the pore system, which is important for simulating the internal frost damage models. 


\section{CHAPTER 3 EXPERIMENTAL TESTS ON CONCRETE AND CEMENT PASTE SAMPLES}

\subsection{Overviews}

In this chapter, Task 3 will be presented for concrete and cement paste specimens preparations followed by experimental tests. The specimen preparations are not only applied for the controlled freeze-thaw test but also SEB tests for validation purposes. Although numerical modeling is the major part in this doctoral study, the concrete and cement paste samples preparations are essential to produce desired and quality results. Hence, a well written plan for mixture designs and sample preparations was accomplished to ensure that the fabricated samples will meet the design constraints and to reach the goals of this doctoral study. Three sizes of specimens were prepared in this doctoral study. First, in macro-scale level, concrete beams and cylindrical concrete specimens were fabricated to perform SEB tests and material property tests, respectively. Cement paste beams were also fabricated to perform controlled freeze-thaw tests. In meso-scale, cement paste samples with diameter of approximately $2.3 \mathrm{~mm}$ were fabricated to perform controlled freeze-thaw test. In micron-scale level, cement paste samples with diameters in the range of $100 \mu \mathrm{m}$ to $200 \mu \mathrm{m}$ were prepared to meet the TXM specifications and to perform controlled freeze-thaw tests. Preparing the TXM samples was the most challenging task as the fabricated cement paste samples are micron scale and require special treatments in fabrication. Through brainstorming and recommendations from experts, the micron scale TXM samples were fabricated successfully by using special glass tubes as the mold. After performing tests on the 
fabricated samples, the microstructure image data were acquired to study the fracture behavior of the tested samples, which will be discussed in Chapter 4 .

\subsection{Concrete and Cement Paste Specimens Preparations}

\subsubsection{Concrete Specimens Preparations}

The mixture design for the concrete specimens was in accordance to the ASTM standards. Table 3-1 highlights ASTM standards (ASTM 2009) that were followed for the concrete mixture designs:

Table 3-1: List of ASTM Standards (ASTM 2009)

\begin{tabular}{|c|c|}
\hline ASTM Standards & Descriptions \\
\hline C $33-07$ & Standard specification for concrete aggregates \\
\hline C 117-04 & $\begin{array}{l}\text { Standard test method for materials finer than } 75 \mu \mathrm{m} \text { (No. 200) } \\
\text { Sieve in mineral aggregates by washing }\end{array}$ \\
\hline C $123-04$ & Standard test method for lightweight particles in aggregates \\
\hline C $127-07$ & $\begin{array}{l}\text { Standard test method for density, relative density (specific } \\
\text { gravity), and absorption of coarse aggregate }\end{array}$ \\
\hline C $136-06$ & $\begin{array}{l}\text { Standard test method for sieve analysis of fine and coarse } \\
\text { aggregates }\end{array}$ \\
\hline C 138/C 138M-08 & $\begin{array}{l}\text { Standard test method for density (unit weight), yield, and air } \\
\text { content (gravimetric) of concrete }\end{array}$ \\
\hline C $143 / \mathrm{C} 143 \mathrm{M}-08$ & Standard test method for slump of hydraulic-cement concrete \\
\hline C $173 / \mathrm{C} 173 \mathrm{M}-08$ & $\begin{array}{l}\text { Standard test method for air content of freshly mixed concrete } \\
\text { by the volumetric method }\end{array}$ \\
\hline C $231-08 b$ & $\begin{array}{l}\text { Standard test method for air content of freshly mixed concrete } \\
\text { by the pressure method }\end{array}$ \\
\hline
\end{tabular}




\begin{tabular}{|c|l|}
\hline C 192/C 192M-07 & $\begin{array}{l}\text { Standard practice for making and curing concrete test specimens } \\
\text { in the laboratory }\end{array}$ \\
\hline C 39/C 39M-05 & $\begin{array}{l}\text { Standard test method for compressive strength of cylindrical } \\
\text { concrete specimens }\end{array}$ \\
\hline
\end{tabular}

Concrete specimens were prepared with a special mixture design. The water/cement ratio $(\mathrm{w} / \mathrm{c})$ was 0.5 to introduce more capillary pores. Table 3-2 listed the gradation of fine and coarse aggregates for the mixture design. It is shown that the largest aggregate used in this doctoral study was $3 / 8 \mathrm{inch}$. The ratio of coarse aggregates to fine aggregates was $60 / 40$. Table 3-3 shows the specific weight of each mixture component and the weight ratio of mixture components with respect to the total weight of the mixture.

Table 3-2: Aggregate sizes and gradation in the mixture design

\begin{tabular}{|c|c|c|c|}
\hline \multirow{2}{*}{ Aggregate Types } & \multicolumn{2}{|c|}{ Sieve Size } & \multirow{2}{*}{$\begin{array}{c}\text { Percent Weight } \\
\text { Passing (\%) }\end{array}$} \\
\cline { 2 - 4 } & Inch & $\mathbf{~ m m}$ & 4 \\
\hline \multirow{3}{*}{ Fine Aggregates } & $\# 50$ & 0.30 & 10 \\
\cline { 2 - 4 } & $\# 30$ & 0.60 & 20 \\
\cline { 2 - 4 } & $\# 16$ & 1.18 & 40 \\
\hline \multirow{3}{*}{ Coarse Aggregates } & $\# 8$ & 2.36 & 49 \\
\cline { 2 - 4 } & $\# 4$ & 4.75 & 73 \\
\cline { 2 - 4 } & $3 / 8^{\prime \prime}$ & 9.50 & 100 \\
\cline { 2 - 4 } & $1 / 2 "$ & 12.5 & \\
\hline
\end{tabular}


Table 3-3: Specific weight and weight ratio of mixture contents

\begin{tabular}{|c|c|c|c|c|}
\hline & $\begin{array}{c}\text { Coarse } \\
\text { Aggregate }\end{array}$ & $\begin{array}{c}\text { Fine } \\
\text { Aggregate }\end{array}$ & $\begin{array}{c}\text { Portland } \\
\text { Cement }\end{array}$ & Water \\
\hline $\begin{array}{c}\text { Specific Weight } \\
\left(\mathbf{l b s} / \mathbf{y d}^{3}\right)\end{array}$ & $1,963.65$ & $1,309.12$ & 594.08 & 297.04 \\
\hline Weight Ratio (\%) & 47.16 & 31.44 & 12.27 & 7.13 \\
\hline
\end{tabular}

With these mixture designs, the air void ratios of the fresh concrete was measured and determined to be $2 \%$ by using the volumetric and pressure method. After the mixtures were mixed, the temperature of fresh concrete was measured and determined to be about $73.0 \mathrm{~F}$. The unit density of fresh concrete was measured to be $156.4 \mathrm{lbs} / \mathrm{ft}^{3}$. The slump test was also conducted with 0.25 inch reduction. In addition, the compressive tests were also performed using Baldwin Material Testing Equipment, as shown in Figure 3-1. The compressive strength for 7, 14 and 28 days of the concrete specimens are as shown in Table 3-4. Overall, a total of six concrete beams for SEB bending tests and six concrete cylinders for compression tests were prepared.

Table 3-4: Compressive strengths of concrete specimens for different curing periods

\begin{tabular}{|c|c|c|c|c|}
\hline & 3 Days & 7 Days & 14 Days & 28 Days \\
\hline Failure Stress (psi) & $2,181.95$ & $3,671.66$ & $5,272.95$ & $6,114.16$ \\
\hline
\end{tabular}


(a)

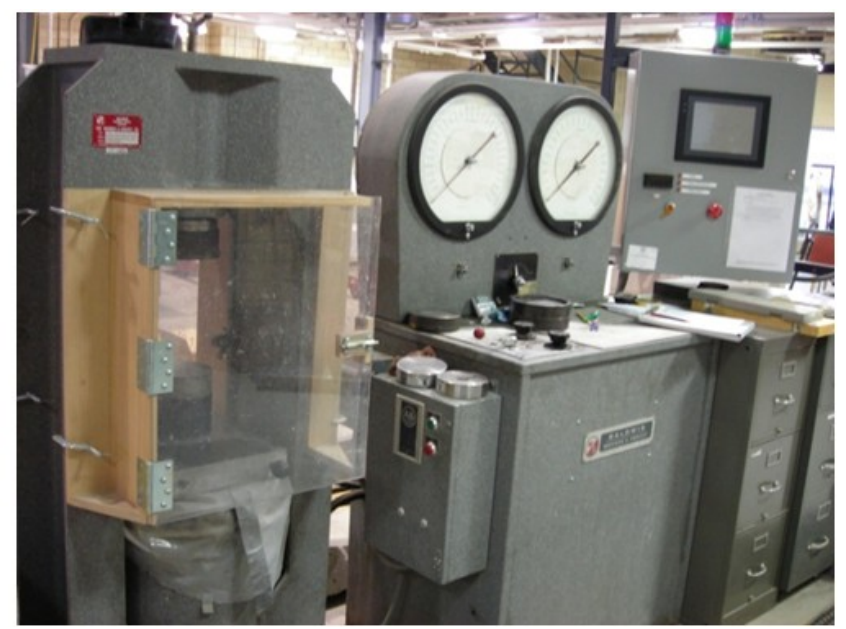

(b)

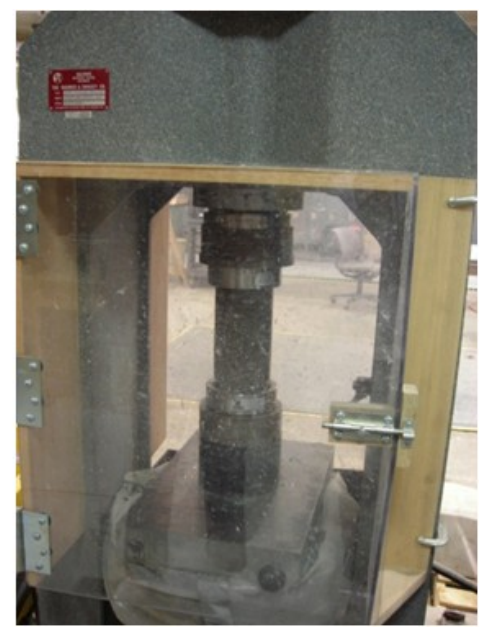

Figure 3-1: Compression test on concrete specimens to determine the failure stress. (a) The Baldwin Material Testing Equipment utilized for performing compression test. (b) A compression test conducted on a concrete specimen.

\subsubsection{Cement Paste Specimens Preparations}

Cement paste specimens were prepared for performing controlled freeze-thaw tests. Three types of specimens were fabricated based on the design constraints for the experimental tests: (1) Freeze-thaw beams (macro-scale), cylindrical specimens (mesoscale), and TXM specimens (micro-scale). The fabrication procedures were in accordance to ASTM C305-06. Rather than using a mixer machine, the cylindrical specimens and TXM specimens were manually mixed because only a small quantity was required. Six mixture designs were prepared for the cement paste freeze-thaw beams, which are listed below:

(1) Beam 1: w/c $=0.45$ without air entrainment

(2) Beam 2: w/c $=0.45$ with air entrainment

(3) Beam 3: w/c $=0.47$ without air entrainment

(4) Beam 4: w/c $=0.47$ with air entrainment 
(5) Beam 5: w/c $=0.50$ without air entrainment

(6) Beam 6: $\mathrm{w} / \mathrm{c}=0.50$ with air entrainment

All of the beams have the standard dimensions of $15.5 \times 4 \times 3$ cubic inches $(39.37$ $\times 10.16 \times 7.62$ cubic centimeters) that fit into the freeze-thaw chamber, as shown in Figure 3-2. Table 3-5 highlights the mixture contents for preparing cement paste freezethaw beams. Upon fabricating the beam specimens, the specimens were let hardened for a day followed by submerging them in lime water for 28 days. The specimens were then air cured for 28 days more before performing the controlled freeze-thaw tests.

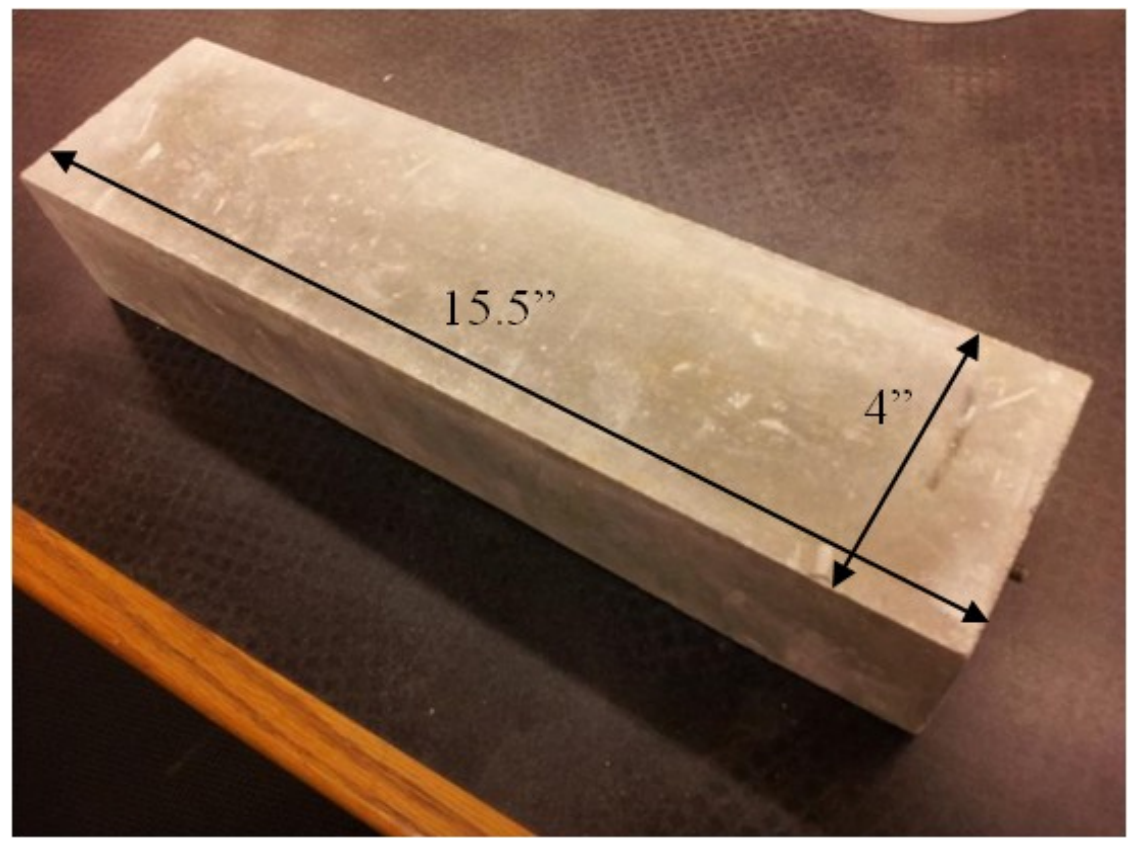

Figure 3-2: A cement paste freeze-thaw beam with 15.5 inches in length, 4 inches in width, and 3 inches in thickness. 
Table 3-5: Mixture contents for preparing cement paste freeze-thaw beams

\begin{tabular}{|c|c|c|c|c|}
\hline \multirow{2}{*}{ W/C } & \multirow{2}{*}{ Volume (in $\mathbf{3}^{\mathbf{3}}$ ) } & \multicolumn{3}{|c|}{ Weight (kg) } \\
\cline { 3 - 5 } $\mathbf{0 . 4 5}$ & 186 & 1.72 & 3.85 & 0.1 \\
\cline { 2 - 5 } & 186 & 1.73 & 3.85 & 0.0 \\
\hline \multirow{2}{*}{$\mathbf{0 . 4 8}$} & 186 & 1.77 & 3.71 & 0.1 \\
\cline { 2 - 5 } & 186 & 1.78 & 3.71 & 0.0 \\
\hline \multirow{2}{*}{$\mathbf{0 . 5 2}$} & 186 & 1.82 & 3.53 & 0.1 \\
\cline { 2 - 5 } & 186 & 1.84 & 3.53 & 0.0 \\
\hline
\end{tabular}

For preparing meso-scale cylindrical cement paste samples, two mixture designs were fabricated. The two designs are $\mathrm{w} / \mathrm{c}=0.45$ and $\mathrm{w} / \mathrm{c}=0.47$. All the cylindrical samples were fabricated by using straws as molds. The straws have an inner diameter of approximately $2.3 \mathrm{~mm}$, as shown in Figure 3-2(a). Upon hardening for a day, the samples were kept in saturated condition before performing the controlled freeze-thaw tests in the freezer chamber. 


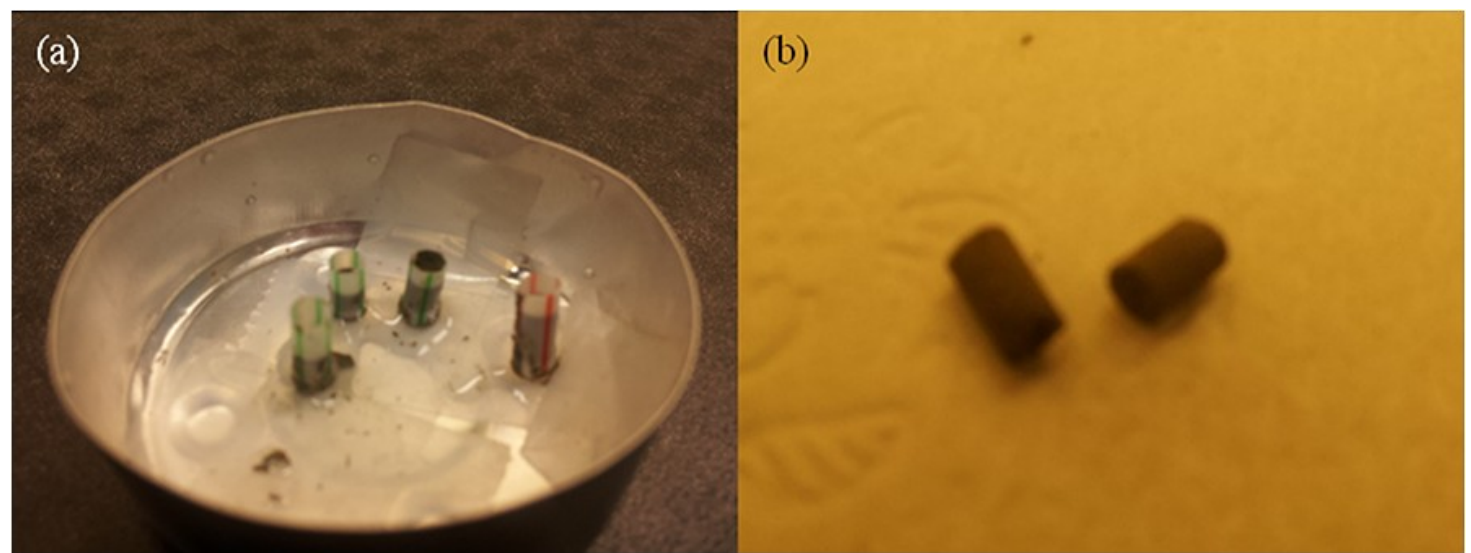

Figure 3-3: Cylindrical cement paste specimen fabrications. (a) The mixed cement paste are filled into straws and let hardened for a day. (b) The hardened cement paste after removing straw molds.

For preparing micron-scale TXM specimens, special glass thin-walled capillary tubes that were designed for X-ray diffraction were applied as the molds. The capillary tubes were purchased from the Charles Supper Company. The tube has a wall thickness of $0.01 \mathrm{~mm}$ and a funnel at one end. The fabricated sample might have diameters in the range from $100 \mu \mathrm{m}$ to $200 \mu \mathrm{m}$ due to the tube is being tapered from one end to another. Three mixture designs for the specimens were prepared with w/c of $0.45,0.47$, and 0.50 . After mixing the cement paste manually, the cement paste was filled into a syringe immediately followed by squeezing the paste into the capillary tube through the tube funnel. Two or three sections were cut immediately after filling the pastes into the capillary tube to prevent the capillary effect that can draw out trapped pastes from the tube. Each section had a length in the range of $2 \mathrm{~mm}$ to $3 \mathrm{~mm}$. The samples were then handled with care to be kept in the saturated condition before performing the controlled freeze-thaw tests in the freezer chamber. 


\subsection{Experimental Tests}

\subsubsection{Single-edge-notched beam (SEB) bending test}

The SEB bending beam test on concrete specimens (Figure 3-4 (Dai et al. 2012)) was performed in accordance to ASTM C 293-08 standard. A middle notch with a depth, $a_{0}$ about 0.5 in was sawn cut, as shown in Figure 3-5 (Ng and Dai 2012). The dimension of the beam was $8 \times 2 \times 2$ cubic inches $(20.32 \times 5.08 \times 5.08$ cubic centimeters $)$ with a span of 6 inches $(15.24 \mathrm{~cm})$. A fixed support was applied an inch $(2.54 \mathrm{~cm})$ away from one edge and a roller support was applied an inch away from another edge of the concrete beam, as shown in Figure 3-5(a). The test was performed with a loading rate of 0.05 inch per minute using Mechanical Testing System (MTS) machine. The test was done when the load reached $80 \%$ of the maximum applied load. Figure $3-5(\mathrm{~b})$ demonstrates the deformed and fractured concrete beam after performing the SEB bending test with the developed crack growth path (as indicated in red line). The images for both beams before and after the SEB bending test were acquired from digital scanning for imaging and fracture simulation purposes, which will be discussed in Chapter 4 and 5, respectively. 


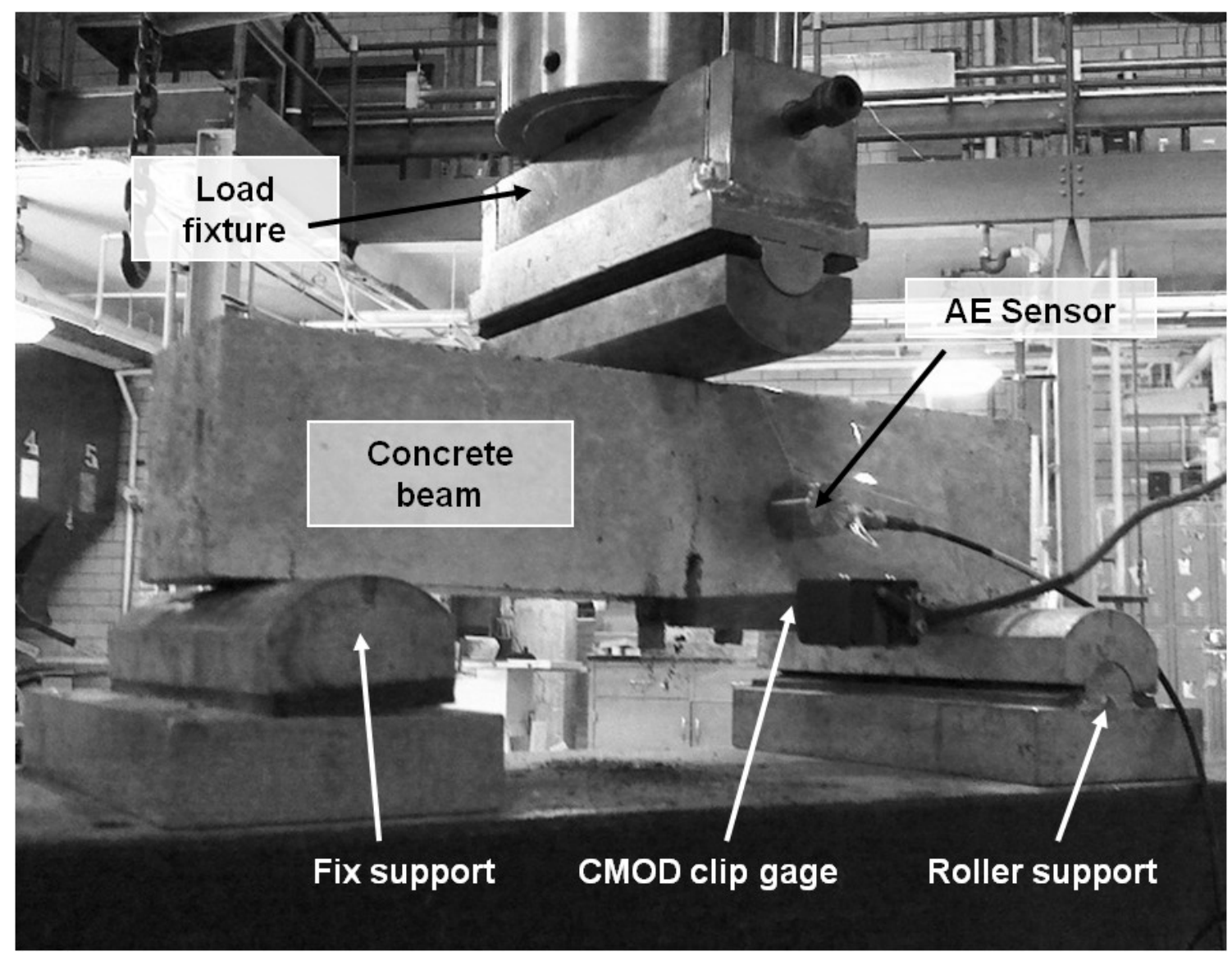

Figure 3-4: The setup for SEB bending test on concrete specimen using MTS machine with load fixture, fix support and roller support (Dai et al. 2012) - see permission in Appendix C6. 

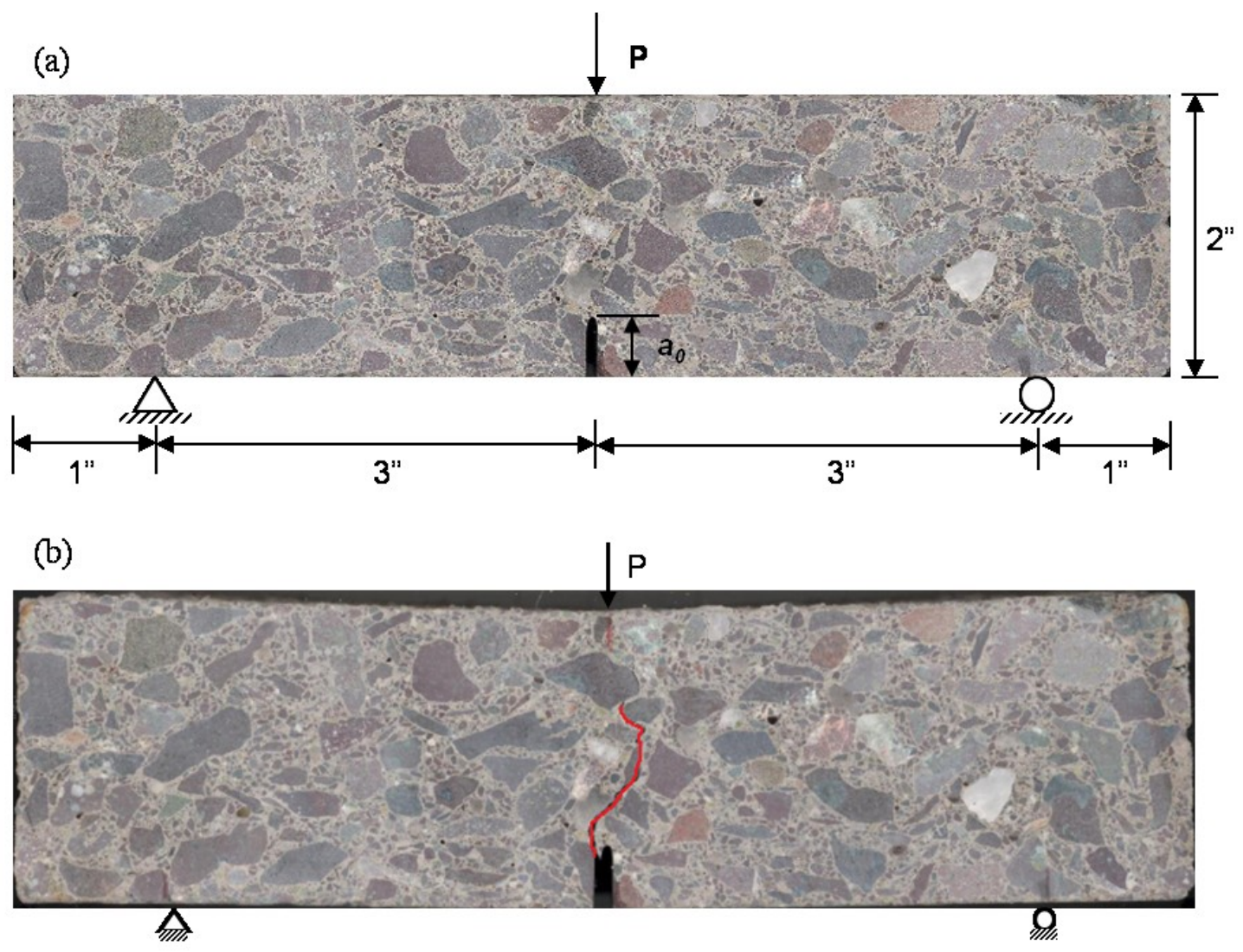

Figure 3-5: SEB bending test on a concrete specimen. (a) Boundary conditions and dimensions for the SEB bending test. (b) Developed crack growth path (red line) after the load reached $80 \%$ of the maximum applied load ( $\mathrm{Ng}$ and Dai 2012) - see permission in Appendix C3.

\subsubsection{Freeze-thaw test}

All fabricated cement paste specimens were used for performing controlled freeze-thaw tests. For cement paste freeze-thaw beams, the ASTM C666 procedure A was performed using a freeze-thaw chamber donated by Michigan Department of Transportation (MDOT), as shown in Figure 3-6(a). Figure 3-6(b) demonstrates six cement paste beam specimens (as highlighted in red square) placed in the chamber before running the controlled freeze-thaw tests. In ASTM C666 procedure A, the beams are 
frozen and thawed in cool water. No measurements were taken after the test as the objective of the freeze-thaw test is to study frost damages to the microstructure of the cement paste specimens after the test. A total of 227 cycles were executed rather than typical 300 cycles. This is because of all the cement paste beams, except for specimens added with air entrainment and w/c of 0.48 and 0.52 , failed or broke into pieces as shown in Figure 3-7. The fracture on the specimen with w/c of 0.45 and air entrainment might be caused by the air entrainment not being distributed evenly. All the specimens were then sectioned to have a thickness of approximately one inch by using a kerosene saw to be prepared as SEM specimens, which will be presented in the next chapter.
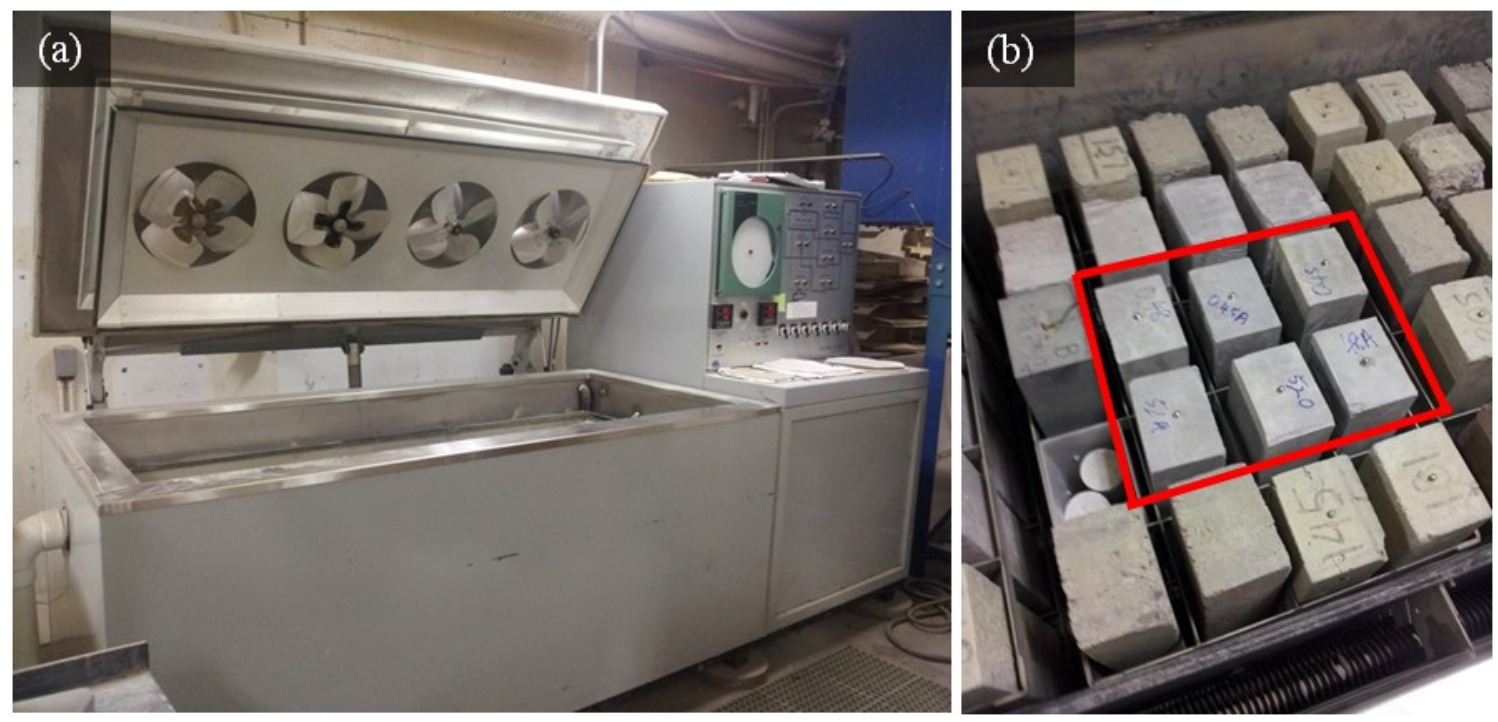

Figure 3-6: Freeze-thaw test on cement paste beam specimens. (a) The freeze-thaw chamber utilized for performing freeze-thaw test using ASTM C666 procedure A. (b) Six cement paste beams with different mixture designs (highlighted in red box) are to be running freeze-thaw test. 


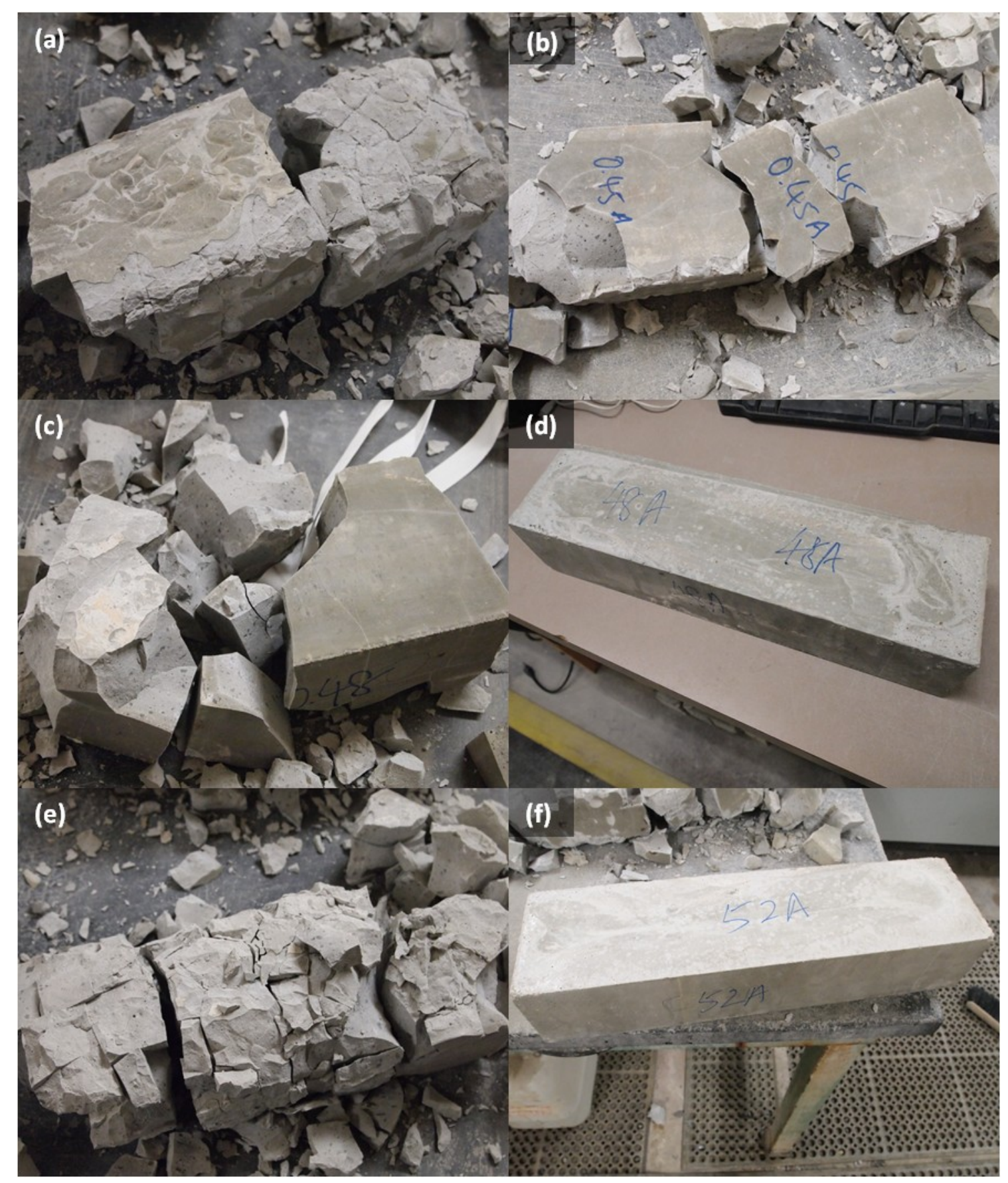

Figure 3-7: Cement paste beam specimens after freeze-thaw test with all specimens encounter complete failure, except for air entrainment specimens with w/c of 0.48 and 0.52. Specimens in (a), (c), and (e) are without air entrainment with w/c of $0.45,0.48$, and 0.52 , respectively. Specimens in (b), (d), and (f) were added with air entrainment with $w / c$ of $0.45,0.48$, and 0.52 respectively. 
On the other hand, the controlled freeze-thaw tests on the cylindrical and TXM cement paste samples were done in accordance to ASTM C666 procedure B. In the ASTM C666 procedure B, the samples are frozen in air and thawed in cool water. Thus, the freeze-thaw test was performed manually using a chest freezer. The lowest temperature that the chest freezer can reach is approximately $-17^{\circ}$. Similarly, no measurements were taken after the test as the objective of the freeze-thaw test is to study the frost damages to the microstructure of the cement paste specimens. All the specimens were kept in the saturated condition to avoid desiccation to occur. The freeze-thaw tests on those samples were carried out with a total of 60 cycles. This is because the miniature size of the specimens can be damaged easily by the crystallization pressure in the pore system. To avoid complete fracture or total failure that occurred on the cement paste beam specimens, the reduced amount of freeze-thaw cycles will allow a clear observation of fracture behavior in the initial stage.

\subsection{SEM Specimens' Preparation}

In this dissertation, only the cylindrical cement paste samples will be presented because the developed cracks are fewer and are identified to be caused by the freezing damage by the crystallization pressure effect. The cement paste freeze-thaw beams are not utilized for analysis because mass fracture cracks and desiccation cracks were observed and were hard to judge whether the damages were due to frost damage or desiccation effect.

In order to obtain quality SEM images, the grinding and polishing procedures on preparing SEM samples were done with care. Figure 3-8 shows the cylindrical cement 
paste specimens that had been polished on Plexiglas and were ready to be observed in the SEM instrument. Seven specimens were observed in total with two different mixture designs. For $\mathrm{w} / \mathrm{c}=0.47$, there are two specimens each for post-freeze-thaw (samples A and B) and pre-freeze-thaw (samples $\mathrm{E}$ and F). Whereas, for $\mathrm{w} / \mathrm{c}=0.45$, there are two specimens for post-freeze-thaw (samples C and D) and a specimen for pre-freeze-thaw (sample G).

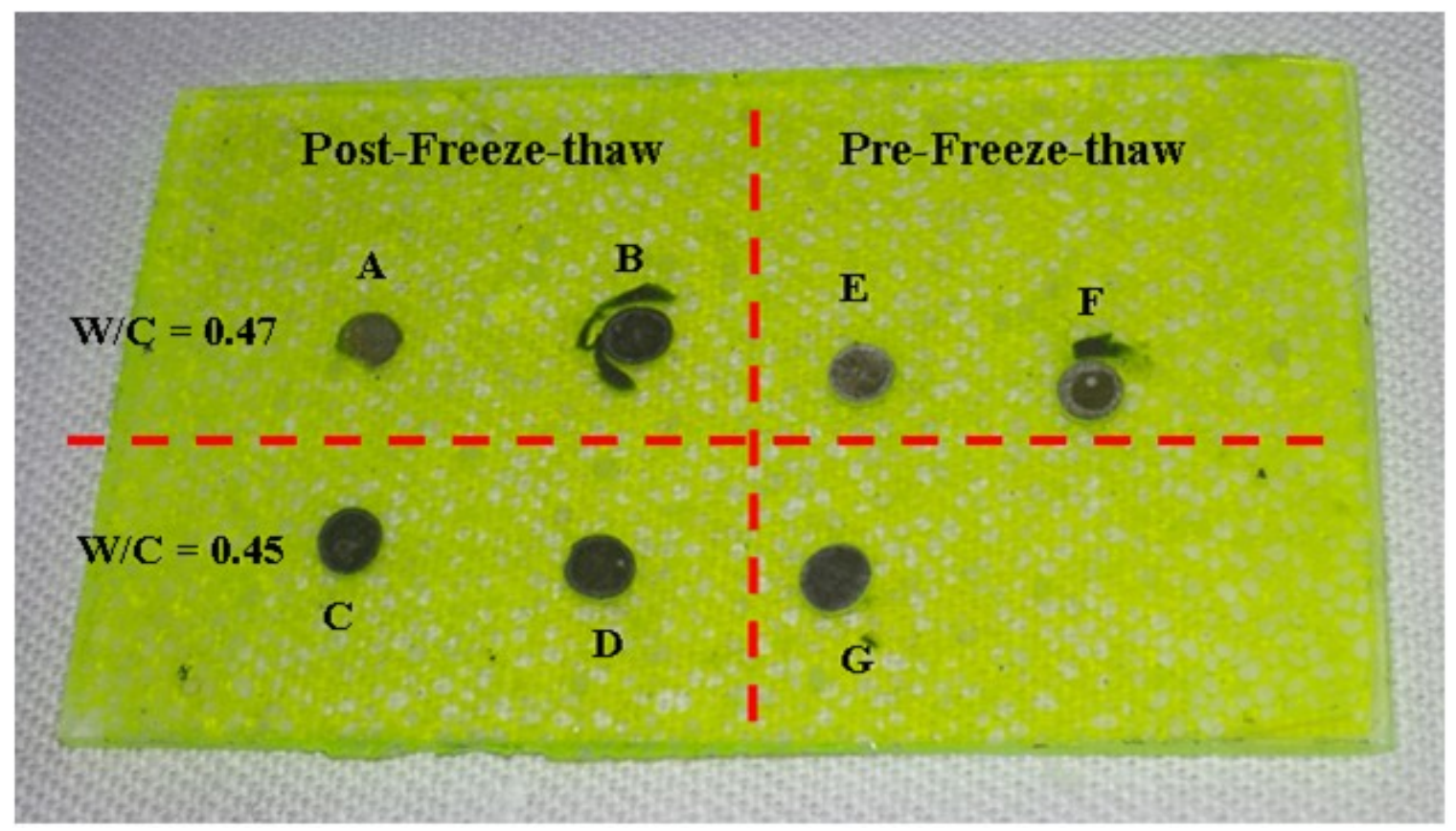

Figure 3-8: The cylindrical cement paste samples on a Plexiglas that were prepared for microstructure study using SEM instrument.

Due to the size and shape of the specimens, a special preparation for SEM specimen was carried out. First, all the specimens were held and adhered fixed on Plexiglas by using JB-Weld, as shown in Figure 3-9(a). After the JB-Weld hardened, the 
surrounding Plexiglas edges were wrapped with a plastic sheet to form a container with approximately 1-inch in thickness. This was done to fill the container with sands to cover the specimens completely, as shown in Figure 3-9(b). The sand was utilized to reduce the amount of fluorescent dyed epoxy to be used.

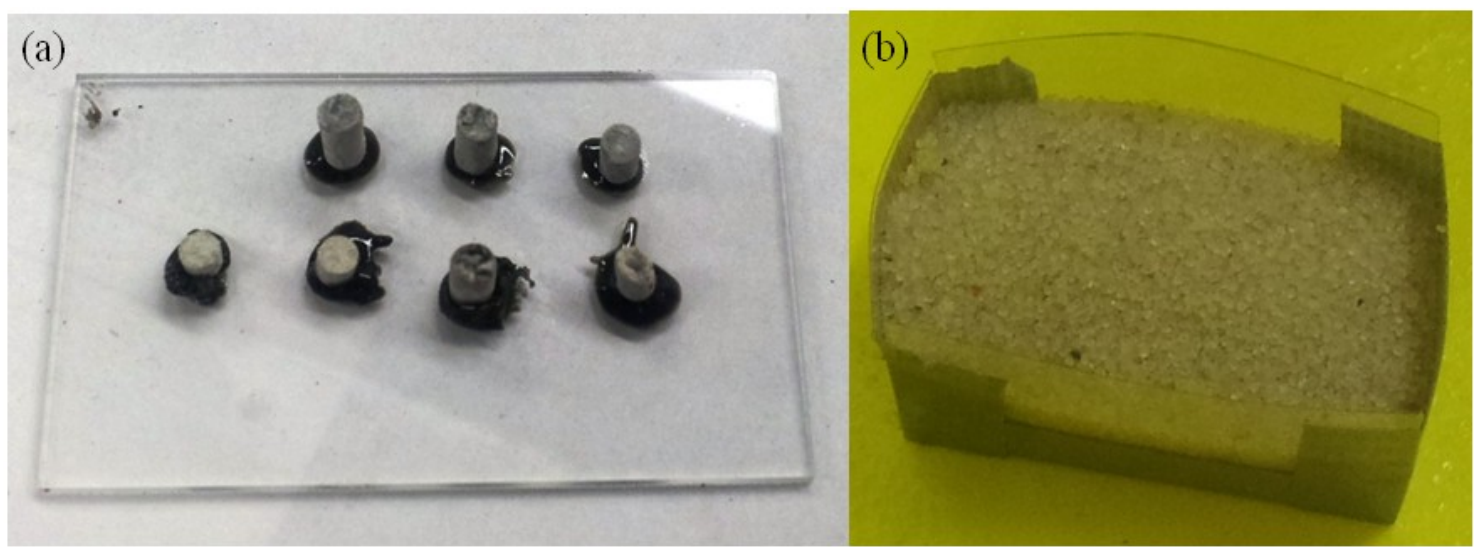

Figure 3-9: Cement paste sample preparation for SEM specimen. (a) The cylindrical cement paste specimens were adhered on a Plexiglas with JB-Weld. (b) A container was made and filled with sands to cover the SEM specimen.

Vacuum impregnation was carried out in a vacuum chamber, as shown in Figure 3-10 (a), to fill any voids or pores in the cement paste specimens with fluorescent dyed epoxy. The vacuum chamber allows all the air that was trapped in the voids or pores in the specimens to be drawn out to ensure that the voids or pores were filled with the fluorescent dyed epoxy. After vacuum impregnation, the SEM specimen was hardened for a day in a dry environment. Figure 3-10 (b) shows the hardened SEM specimen that was ready to be sawn cut to a thin slice. Figure 3-11 shows the kerosene saw machine that was used for cutting SEM specimen into a thin slice. The SEM specimen was sawn 
cut successfully with care by utilizing the vacuum system that held the specimen on the Plexiglas face in the kerosene saw machine. Figure 3-8 shows the sawn cut SEM specimen in thin section.

(a)

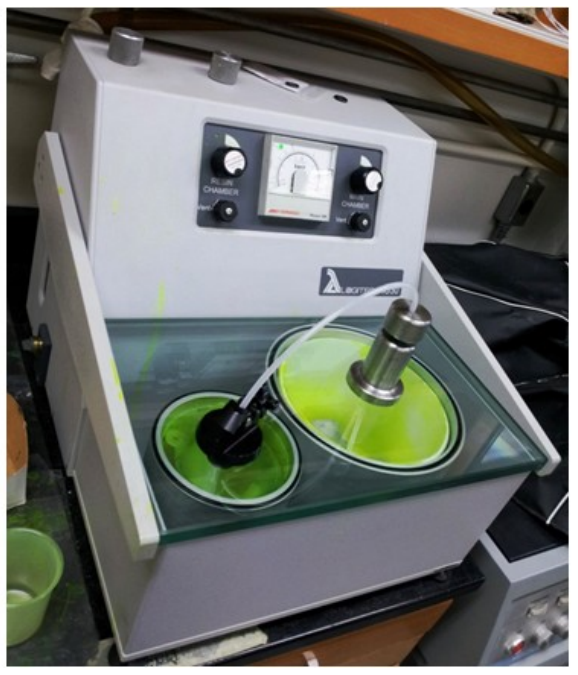

(b)

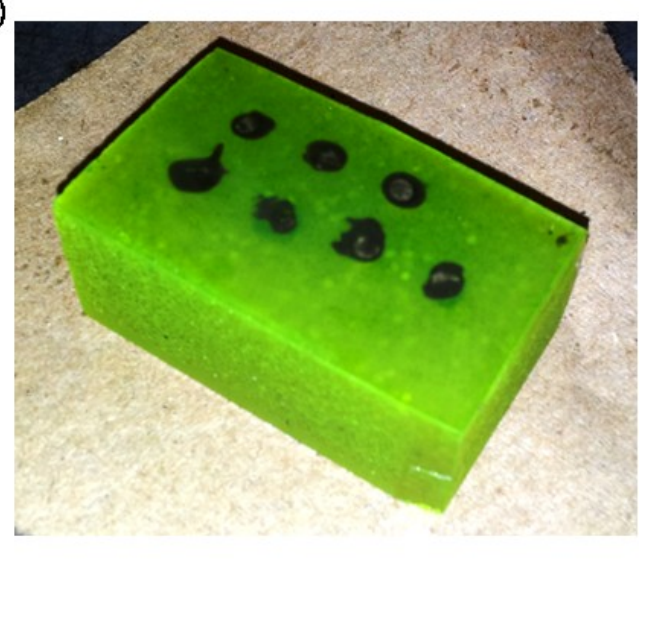

Figure 3-10: Vacuum impregnation on SEM specimen. (a) A vacuum chamber used for performing vacuum impregnation. (b) The SEM specimen with hardened fluorescent dyed epoxy. 


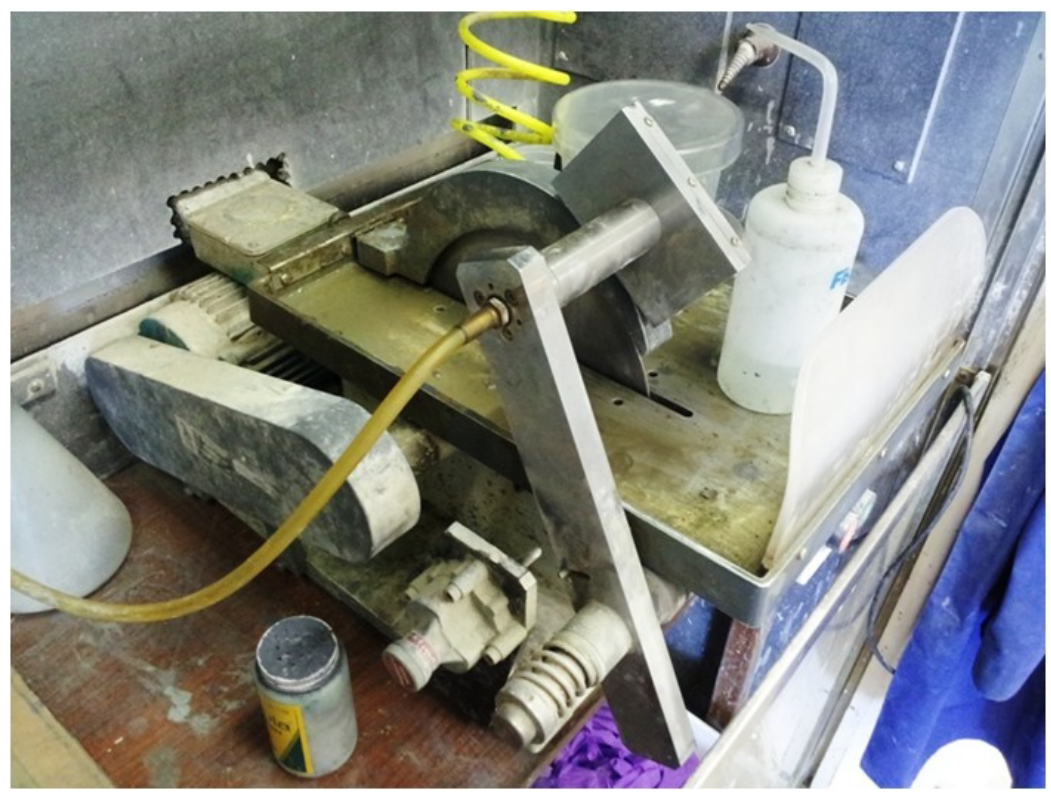

Figure 3-11: Kerosene saw machine that was used to cut the SEM specimen into a thin slice, as illustrated in Figure 3-8.

Next, the thin slice SEM specimen was polished to allow a crisp image to be observed through the SEM instrument. The procedure was done first by polishing the sawn cut surface manually using 600 grits of silicon carbide followed by 1000 grits on a glass surface. Approximately 20 to 40 laps were carried out during the polishing. The polished surface was observed at the interval to ensure that the surface thickness was not removed too much. The polishing procedures were continued by using diamond fine grits in the order of $9 \mu, 6 \mu, 3 \mu, 1 \mu$, and $0.25 \mu$ grits on polisher lapping machines. For $9 \mu, 6 \mu$, and $3 \mu$ grits, the SEM specimen was held fixed in a jig and polished manually on the polisher lapping machine with a rotational speed of $250 \mathrm{rpm}$, as shown in Figure 3-12(a). Then, the polisher lapping machine with vibrated table in Figure 3-12(b) and (c) was utilized to polish the SEM specimen by using $1 \mu$, and $0.25 \mu$ grits of diamond, respectively. A fixture was applied to push down the SEM specimen in both polisher 
lapping machines. The vibration mode enabled the specimen that was held in the fixture to circulate around the table of the machine and thus, polished the specimen for 20 minutes. Throughout the polishing steps, kerosene was used to clean the SEM specimen. The polished SEM samples were then coated with a layer of carbon film to carry away the charging electrons prior using the SEM machine.

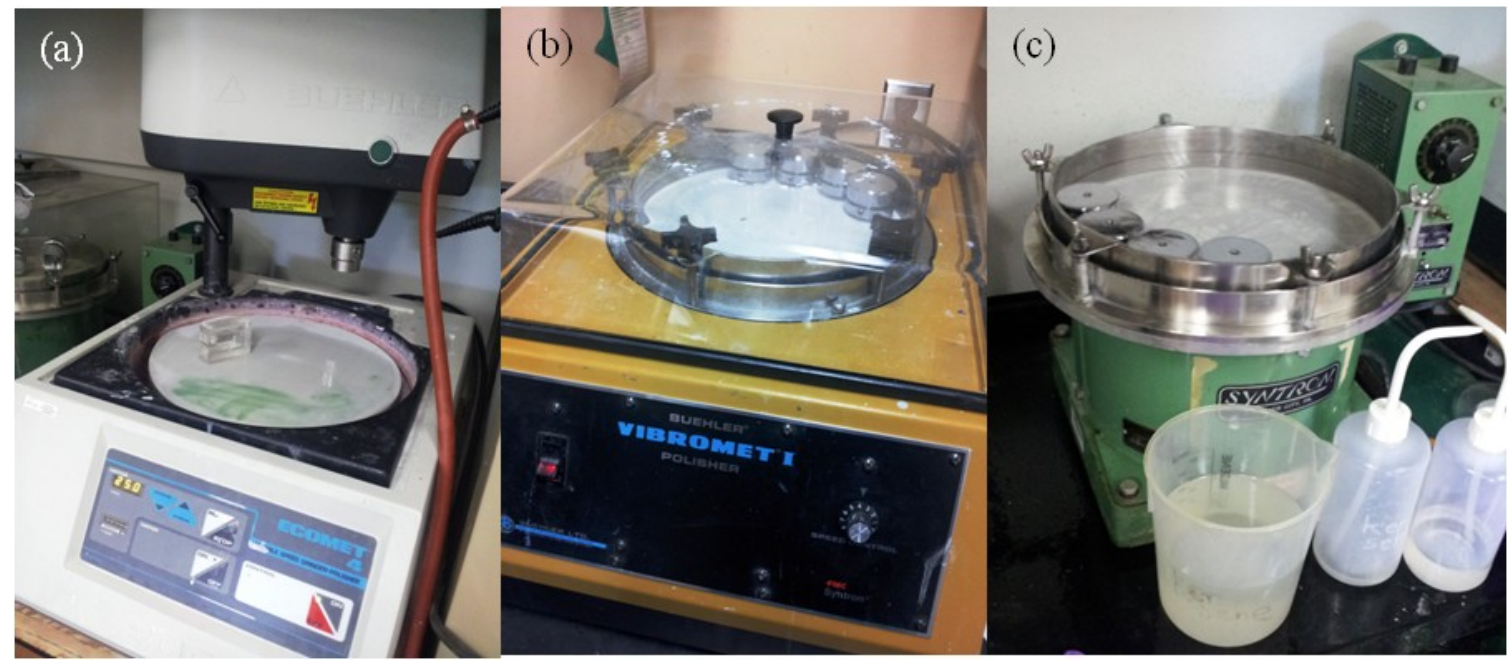

Figure 3-12: The polisher lapping machines that were used to polish the SEM specimen. (a) The polisher lapping machine that used $9 \mu, 6 \mu$, and $3 \mu$ grits. (b) The polisher lapping machine that used $1 \mu$, and (c) The polisher lapping machine that used $0.25 \mu$.

\subsection{Microstructure Study using TXM}

The TXM facility is located at the Advanced Photon Source (http://www.aps.anl.gov/Users/Prospective/) in Argonne National Laboratory, Illinois. The TXM machine, as shown in Figure 3-13, is at the beam line of 32-ID. The TXM technique was applied to study the cement paste microstructure because of some advantages, such as fast image acquisition with $30-\mathrm{nm}$ resolutions. 


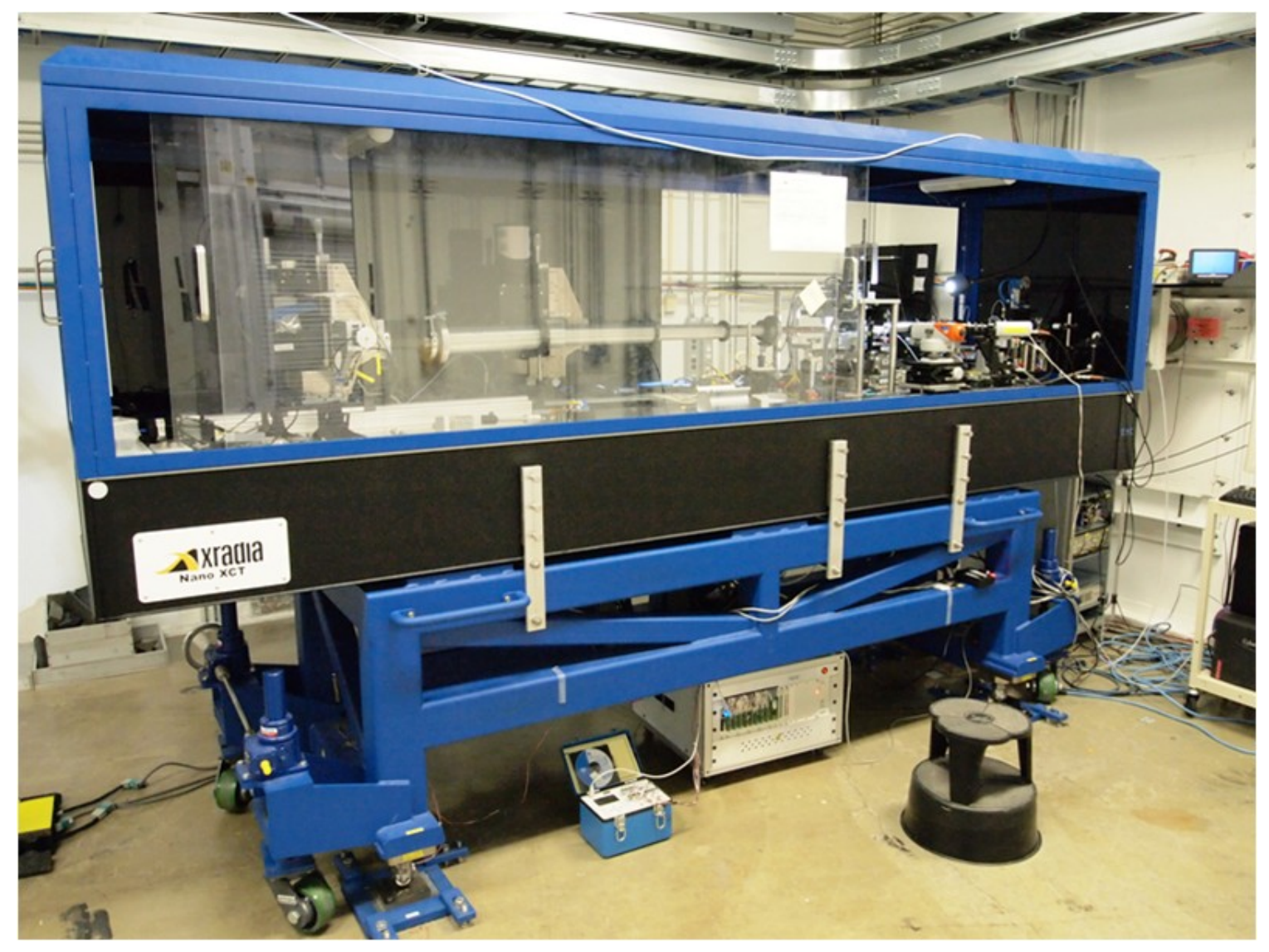

Figure 3-13: The TXM machine at the beam line of 32-ID that was used to study the cement paste microstructure.

In fabricating cement paste samples, w/c of 0.45 and 0.47 were prepared to generate more capillary pores by following the standard of ASTM C305. Due to the field of view from the TXM was $25 \mu \mathrm{m}$, a cross-section diameter of $50 \mu \mathrm{m}$ to $200 \mu \mathrm{m}$ was allowed for cylindrical specimens. Thus, a special procedure was carried out to meet the specimen size constraint. This was done by injecting the fresh cement paste into special glass thin-walled capillary tube with an inner diameter of $0.08 \mathrm{~mm}$ and a wall thickness of $0.01 \mathrm{~mm}$. The special glass capillary tube was selected as it was designed for X-ray 
diffraction. This tube has high transmission percentage that limits the loss of beam into the cement specimen for photon energy at $10 \mathrm{keV}$. Upon injecting the fresh cement paste into the tube, the specimen was sectioned immediately to a length of $1 \mathrm{~mm}$ to avoid capillary effect occurred. The short section allowed the cement paste to absorb water for hydration. The cement paste specimens molded with glass tubes were cured with water for about 28 days. After curing processes, the hardened cement paste specimens in the capillary tubes were kept in saturated conditions for 28 days to fill up the capillary pores with water before performing the freeze-thaw tests.

In order to view the microstructure in TXM without any obstacle, the cement paste specimen was removed from the mold of capillary tube with care. This was done by using razor blade to trim off the capillary tube wall slowly until the tube wall broke. The cement paste specimen that was removed from the mold was adhered on a pin by using clay, as shown in Figure 3-14(a). The top end of the specimen was then welded with a small amount of gold (Figure 3-14(b)) for alignment purpose. The pin with specimen was then held on a fixture before operating the TXM instrument. A X-ray energy of $9 \mathrm{keV}$, an angular range of $180^{\circ}$ for $2 \mathrm{D}$ projection images, and an exposure time of 1 second / 2-D image (15 minutes for data set) were employed to operate TXM instrument to acquire the specimen microstructure images. 

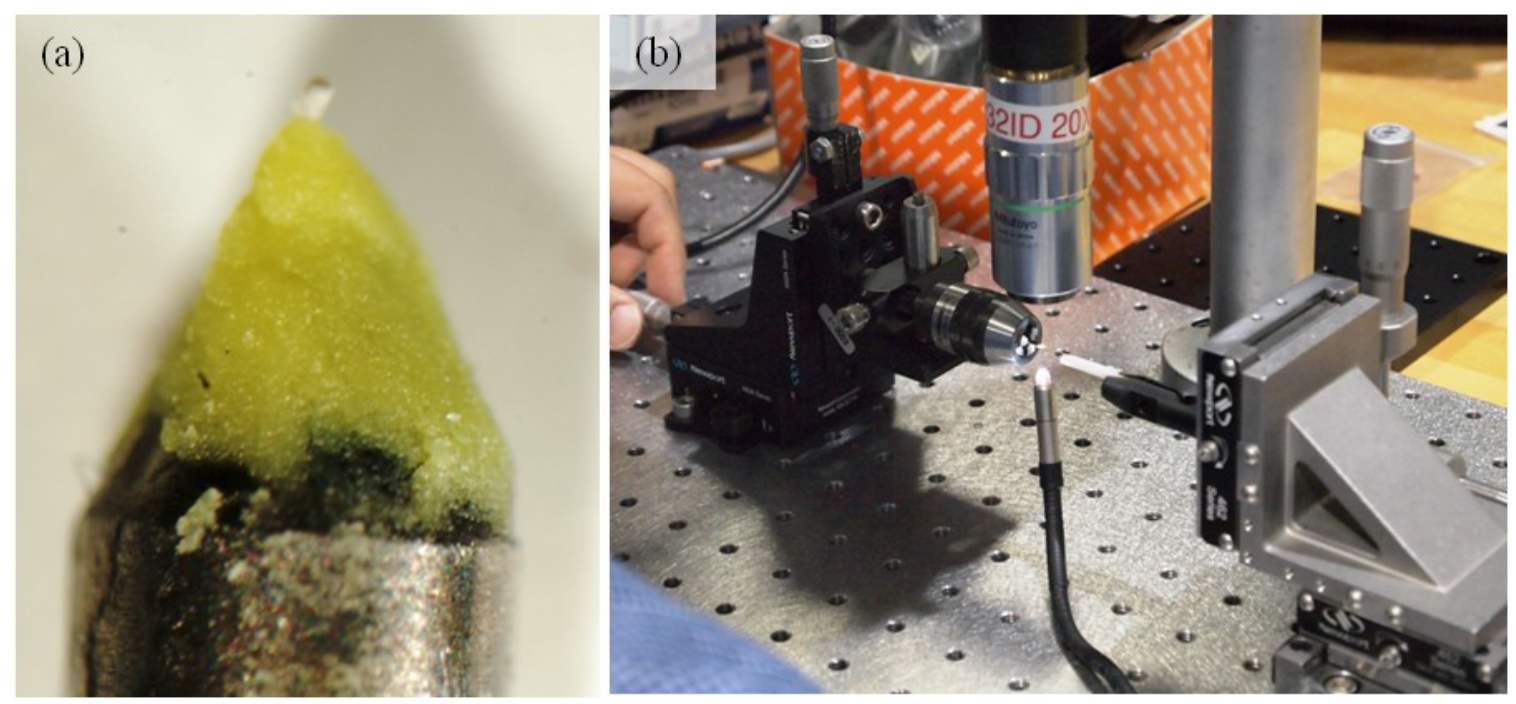

Figure 3-14: Specimen preparation before operating the TXM machine. (a) Cement paste that was adhered on pin by using clay, followed by (b) welding with a small amount of gold for alignment purpose.

\subsection{Concluding Remarks}

Well-planned mixture designs for concrete and cement paste sample preparations are very important for this doctoral research study. This is because the fabricated samples geometries compositions are fixed and cannot be altered to meet specific constraints. Thus, well-planned mixture designs will allow the research project to be carried out smoothly and successfully to avoid extending the project's period and increasing costs, such as materials and services.

All the samples were fabricated in accordance to the ASTM standards at the Benedict Laboratory in MTU. Material property tests, such as the compression test to determine the failure strength, were conducted in accordance to the ASTM standards in the laboratory. All fabricated samples met the planed mixture design requirements and 
test constraints of the laboratory testing equipment, such as SEB bending test and freezethaw test. Hence, all tests were successfully performed and desired results were obtained.

The most challenging laboratory work is to prepare the micro scale cement paste samples because there are no standardized miniature size sample preparations to be followed. Through brainstorming and discussions with experts, all the micro scale cement paste samples were fabricated successfully and met the design constraints of the laboratory instruments, such as the TXM machines.

The freeze-thaw tests had mixed outcomes. The cement paste beam samples experienced severe damage before reaching 300 freeze-thaw cycles. The outcome had provided a guideline to perform the controlled freeze-thaw test on miniature size cement paste samples with fewer cycles. The outcome was acceptable with light damages on the samples' microstructure, which is expected to be mainly caused by the frost damage due to crystallization pressure. Also, the SEM specimen preparations on cement paste samples were successfully, carried out the images of the microstructure can be clearly seen. 


\section{CHAPTER 4 IMAGE DATA ACQUISITION AND ANALYSIS ON CONCRETE AND CEMENT PASTE SAMPLES}

\subsection{Overviews}

The successful specimen preparations and laboratory tests were used to run image analysis to study the microstructure of concrete and cement paste samples. Thus, image data acquisitions and processing were carried out for analyzing the fracture behaviors of the samples. Several image data acquisition techniques had been employed in this doctoral study, depending on the size of the specimens and the applications of the instruments. For SEB test on concrete specimens, the microstructure images were captured through a high resolution scanner. The images were processed by using ImageJ (http://rsb.info.nih.gov/ij/) and Paint to remove noise and to change the image size to fit to the developed numerical models. The processed image data were then transferred and read in MATLAB to construct meshes and element phases based on the predefined threshold magnitudes. The constructed model was used to perform in numerical modeling with the XFEM and bilinear CZM techniques.

The SEM technique was performed on cylindrical cement paste specimens to observe the damage behaviors from the freeze-thaw tests. The acquired SEM images were processed in ImageJ for noise reduction and cropped to obtain desired image section for performing 3-D image reconstruction. The 3-D image reconstruction technique was developed by Mr. Dale Bentz in NIST. The technique was mainly used to develop 3-D microstructure with two phases; they are the pore and matrix phases. Both 2-D and 3-D 
image data were used in numerical modeling by using bilinear CZM technique. For TXM specimens, the 2-D image slices were stacked to construct 3-D microstructure image. With predefined threshold values, the pore structure for the cement paste was constructed. The material phases for both SEM and TXM specimens were identified through the density of the materials. The successful image acquisition and numerical model construction will be used for validation purposes, which will be presented in Chapter 6.

\subsection{Image Analysis on Concrete Samples}

As shown in Figure 4-1(a), the concrete beam had dimensions of $5.08 \times 5.08 \times$ $20.32 \mathrm{~cm}^{3}$ which was in accordance to ASTM C 293-08 standard with a notch depth, $a_{0}$ of approximately $1.27 \mathrm{~cm}(\mathrm{Ng}$ and Dai 2012). In order to reduce the computation time, the scanned specimen image was scaled down to $30 \%$ and only the fracture domain (highlighted in the red box in Figure 4-1(a)) was selected for performing image data processing. The section of the image has dimensions of $64 \times 120$ pixels or $2.71 \times 5.08$

$\mathrm{cm}^{2}$. That is, each pixel was made of approximately $423 \mu \mathrm{m} \times 423 \mu \mathrm{m}$. The portion of interested fracture zone was image-processed and converted to binary image format through ImageJ. This is because the format is readable in MATLAB, which was employed to construct meshes. By employing a grayscale threshold, the cement matrix phase and aggregate phase were generated. As shown in Figure 4-1(b). That is, the cement phase and aggregate have intensities magnitude of 0 (Black) and 255 (white), respectively. 


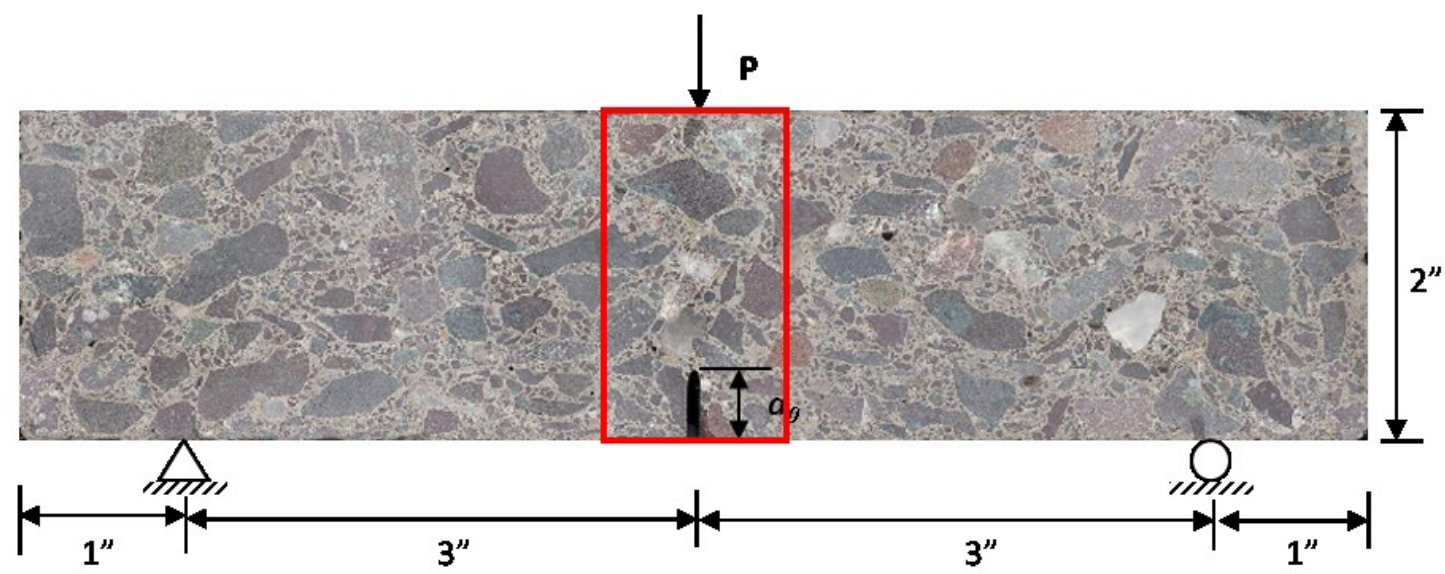

(a)

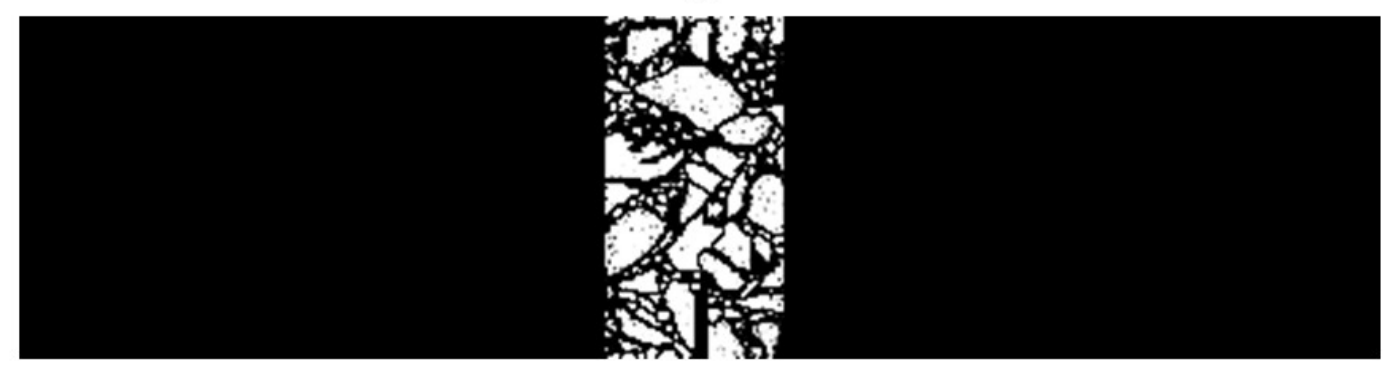

(b)

Figure 4-1: Image processing on a SEB concrete sample. (a) The concrete beam sample was scanned using high resolution scanner and the image was reduced to $30 \%$ of its original size. (b) The scanned image was converted to binary image and threshold was applied to differentiate between cement mastic and aggregate phases ( $\mathrm{Ng}$ and Dai 2012) - see permission in Appendix C3.

Further imaging processing was performed to reduce the image noise by using segmentation, smoothing, and sharpening techniques by using the ImageJ. The connected aggregates were separated aided by Paint to create individual aggregates. Since the developed XFEM technique can only simulate regular inclusion geometries, such as circular and elliptical shapes, the irregular shapes of the aggregate in the actual image were converted to idealized regular elliptical geometries. This was done by employing the digital-sieving technique on the image by using a MATLAB program developed by Dai (Dai et al. 2005), which can be found in the DIPimage Toolbox for MATLAB 
(2010). The aggregates were digitally sieved with 14-pixel area size or an actual sieving aggregate size about $1.8 \mathrm{~mm}$. After obtaining the boundary pixels of each aggregate, a least-square, ellipse-fitting algorithm was used to generate fitted ellipse for each sieved aggregate as shown in Figure 4-2(a) (Ng and Dai 2012). The geometry of the fitted ellipses, such as the center coordinate, major and minor axes dimensions, and orientation, were then input to the XFEM program for generating inclusion enrichment as shown in Figure 4-2(b) (Ng and Dai 2012).

In addition to the XFEM technique, the edited scanned image of the concrete beam was also utilized for performing bilinear CZM fracture simulation. Rather than generating idealized geometries and shapes for the aggregate, the aggregates in the bilinear CZM model were represented with the actual geometries and shapes. This was done by importing the image data from the edited scanned image to the developed bilinear CZM program in MATLAB. The aggregate and cement matrix phases were constructed based on the intensity magnitudes in the image data. As shown in Figure 4-2(c), the fracture zone was constructed with cement matrix and aggregate phases that were assigned with red and black colors, respectively. The green color zone was constructed as a homogeneous concrete phase. Thus, there are three phases altogether in the SEB test bilinear CZM model. 


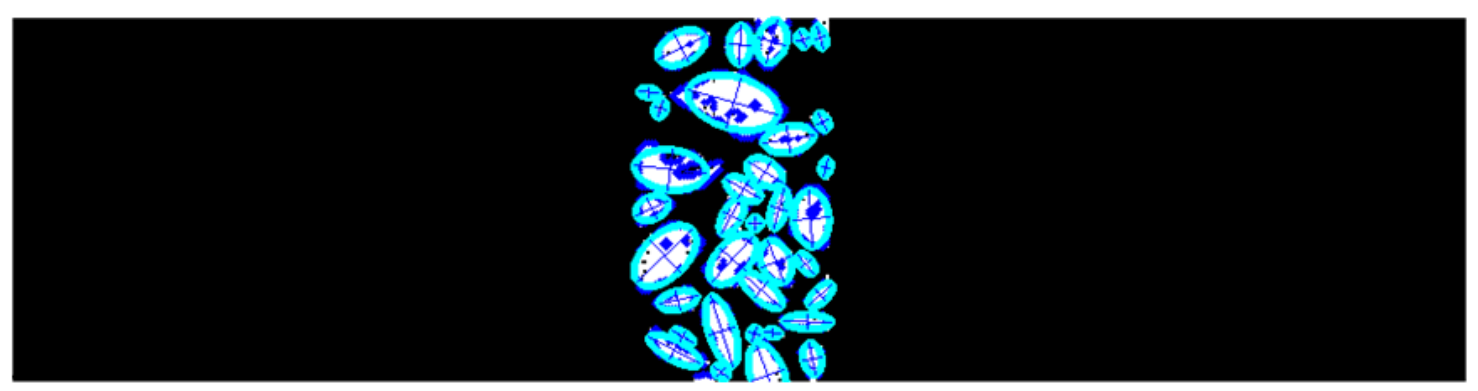

(a)

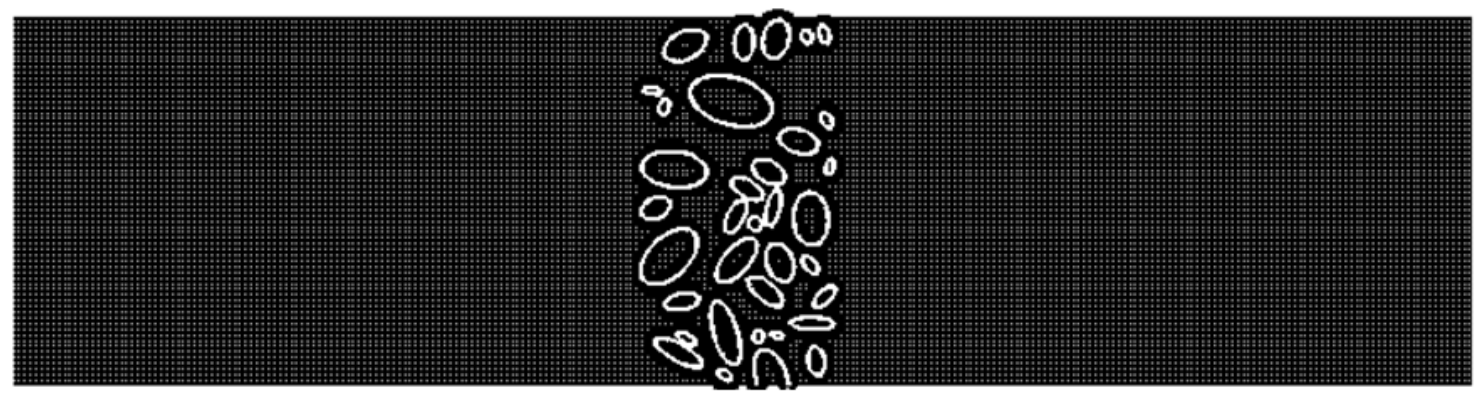

(b)

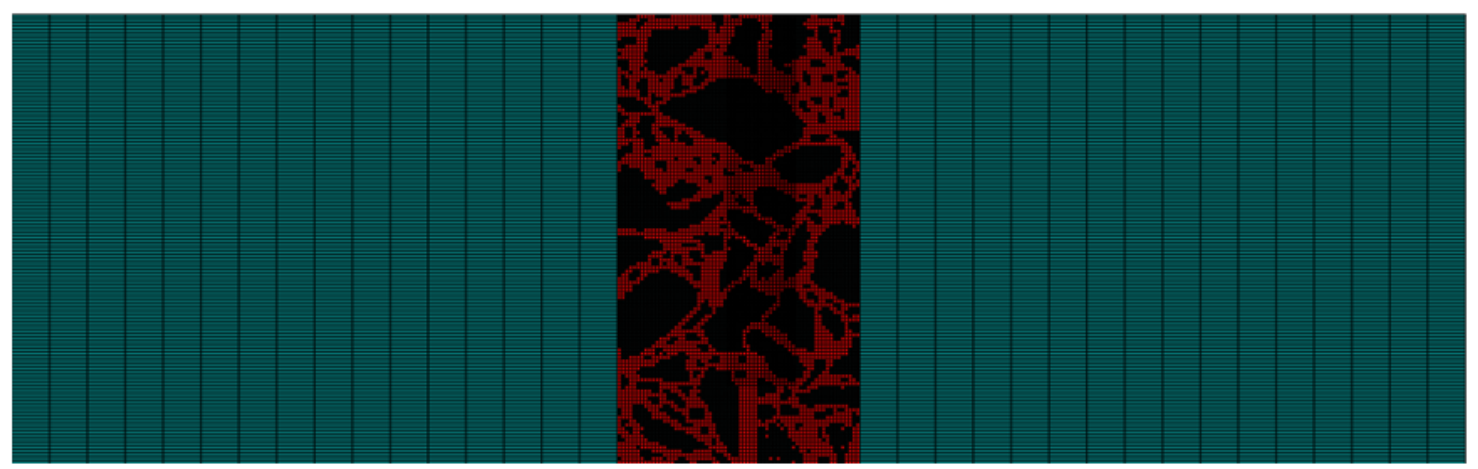

(c)

Figure 4-2: Digital sample generation on the concrete beam from Figure 4-1 for the XFEM and CZM models. (a) Imaging processing and ellipse fitting techniques applied in the fracture domain, and (b) fitted ellipses in the finite element mesh plot ( $\mathrm{Ng}$ and Dai 2012) - see permission in Appendix C3. (c) A bilinear CZM model of the concrete beam was developed that follows the actual aggregates' geometries and sizes.

\subsection{Image Analysis on SEM Samples}

The SEM cement paste sample images were used to perform fracture simulations by using the bilinear CZM technique. Both undamaged and damaged samples were 
utilized to investigate the fracture behavior in the cement paste microstructures. For undamaged samples, the CT test model was constructed and modeled. The model was performed to predict the crack grow path and to prove the capability of the bilinear CZM technique to simulate cracks in a porous media. Whereas, the crystallization pressure effect was modeled on the damaged sample after the freeze-thaw test to validate the simulation results.

The digital sample for the CT test was generated from the SEM image of an undamaged cement paste sample with a w/c ratio of 0.47, as shown in Figure 4-3(a). A square image section (indicated with a red box as shown in Figure 4-3(a)) with a size of $100 \times 100$ pixel or a dimension of $9.6 \times 9.6 \mu \mathrm{m}$ was chosen. A median filter of 1.5 pixels radius was employed to reduce the image noise by using ImageJ. The processed image was oriented to 240 degrees clockwise to align the pores along the straight line as shown in the figure. The grayscale colors of black, dark gray and light gray represent the pore, hydrated cement, and unhydrated cement phases of the cement sample, respectively. 


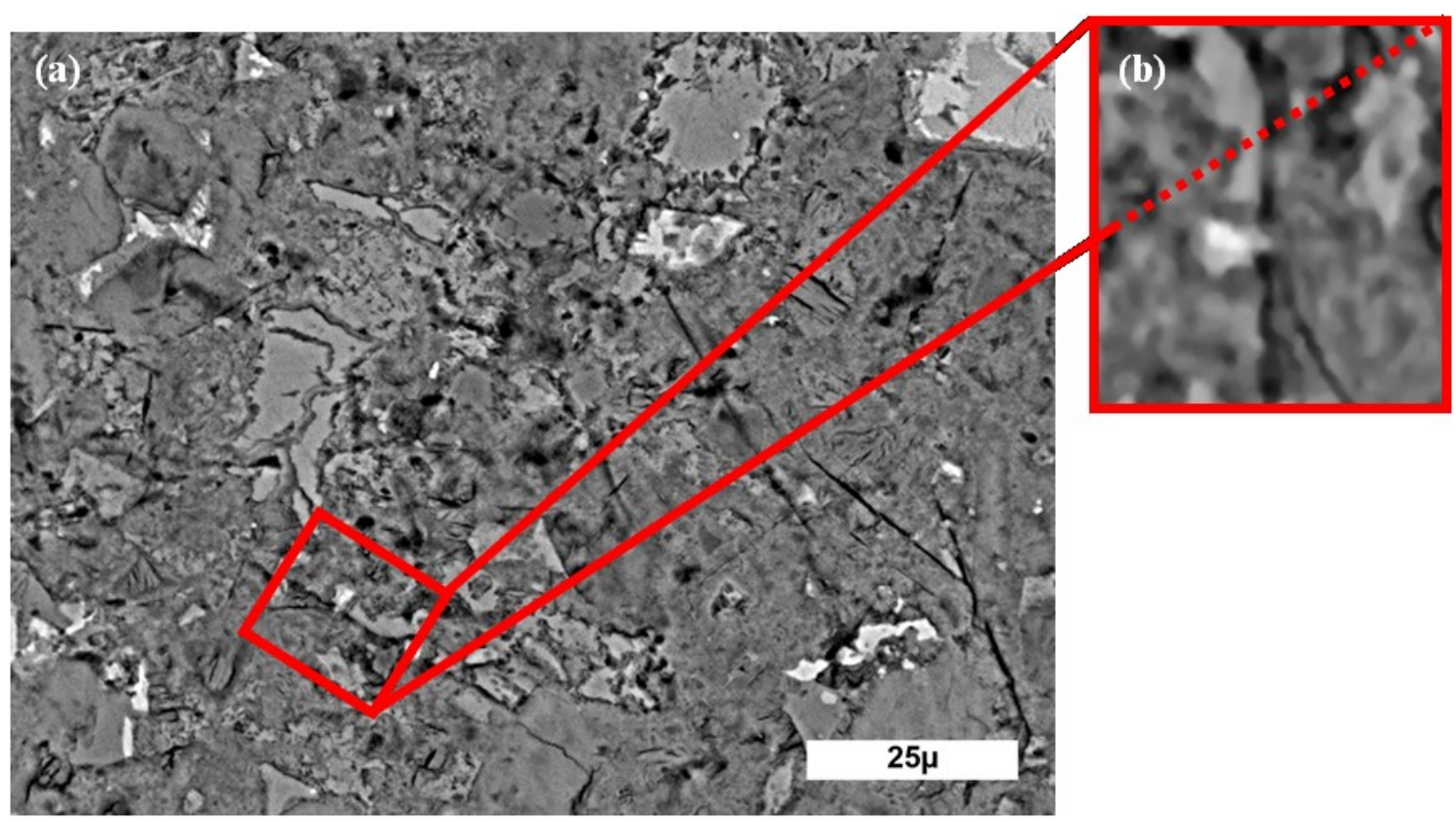

Figure 4-3: A digital SEM image of an undamaged cement paste sample with $\mathrm{w} / \mathrm{c}=0.47$ was processed to generate fracture model. (a) A section of $100 \times 100$ pixels with pore structures was selected, and (b) the image was processed for noise reduction.

The image data was read using a developed MATLAB program to construct the CT model. Three phases were constructed in the model based on the grayscale intensity magnitudes. For hydrated cement phase, the grayscale intensity magnitude ranges between 50 and 130 were assigned. Whereas, the grayscale intensity magnitudes for pore and unhydrated cement phase were assigned below 50 and above 130, respectively. These threshold values were chosen to best represent the phases in the cement sample. Figure 4-4 illustrates the developed FE model for CT test with three phases. That is, the colors of red, black, and white represent the hydrated cement, unhydrated cement, and pore phases, respectively. The filtered image was then edited to create two holes with diameter of 10 pixels for the applied pressure and fixed support. The hydrated cement phase has an elastic modulus and Poisson's ratio of $22 \mathrm{GPa}$ and 0.25 (Liu et al. 2011), respectively. 
Whereas, the unhydrated cement phase has an elastic modulus and Poisson's ratio of 135 GPa and 0.35 (Liu et al. 2011), respectively.

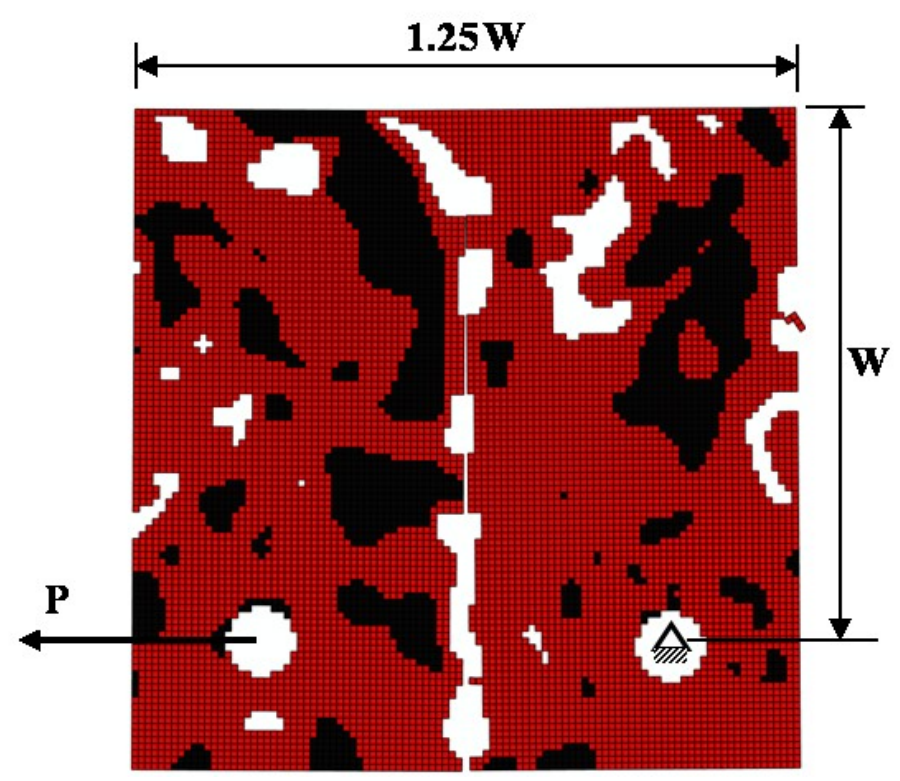

Figure 4-4: The constructed FE model of $\mathrm{CT}$ test with standard dimensions and boundary conditions.

In constructing the FE mesh, the 4-node bilinear interface element and plane stress CPS4 was applied for interface cohesive elements and constitutive elements. Each constitutive element represents one pixel size of $0.096 \mathrm{um} \times 0.096 \mu \mathrm{m}$ in the SEM image. Thus, the CT FE model was made of 100 elements both in columns and rows. An initial crack length of 22 elements was defined with free constrains (without CZM elements) in the middle section of the model. The CZM elements were defined along the central line and above the initial crack tip point in the digital sample. The CZM properties were defined based on the interface between adjacent elements. For hydrated cement phase, the fracture energy and the tensile strength were defined as $37.5 \mathrm{~J} / \mathrm{m}^{2}$ and $0.15 \mathrm{GPa}$, 
respectively. Whereas for unhydrated cement phase, the fracture energy and the tensile strength were defined as $14 \mathrm{~J} / \mathrm{m}$ and $0.15 \mathrm{GPa}$, respectively. Since the interface bonding between hydrated and unhydrated cement phase was lower than the other phases, the tensile strength and fracture energy were defined as $0.05 \mathrm{GPa}$ and $37.5 \mathrm{~J} / \mathrm{m}^{2}$, respectively. To simulate the CT fracture behavior, the displacement load of $1 \mu \mathrm{m}$ was applied in one hole of the digital sample.

Next, the original digital SEM image of a damaged cement paste after freeze-thaw test with $w / c=0.47$ was utilized to perform fracture simulation due to crystallization pressure, as shown in Figure 4-5. A rectangular section with an image size of 100 pixel $\times 120$ pixel and a dimension of $9.6 \mathrm{um} \times 11.5 \mu \mathrm{m}$ was chosen and oriented to 143 degrees clockwise for CZM fracture simulation, as shown in Figure 4-6. A median filter of 1.5 pixels radius was employed to reduce the image noise by using ImageJ. The filtered image was then edited to patch the existing crack zone between two pores, as shown in the circled section of Figure 4-5(b). The resulting patched area (Figure 4-5 (c)) will be defined with CZM elements for the model simulation. It is expected that crack will developed to connect these two pores due to crystallization pressure effects.

The grayscale colors of black, dark gray and light gray or white represent the pore, hydrated cement, and unhydrated cement phases, respectively. The threshold intensity values were chosen to identify these phases in the cement paste sample, as mentioned in the previous section. The image data were read into the developed MATLAB program to construct the FE model. Similar to the CT model, two types of constitutive elements (cement matrix and aggregates) were constructed in the model. The 
colors of red, black, and white represent the finite elements of hydrated cement, unhydrated cement, and pore phases as shown in Figure 4-6.

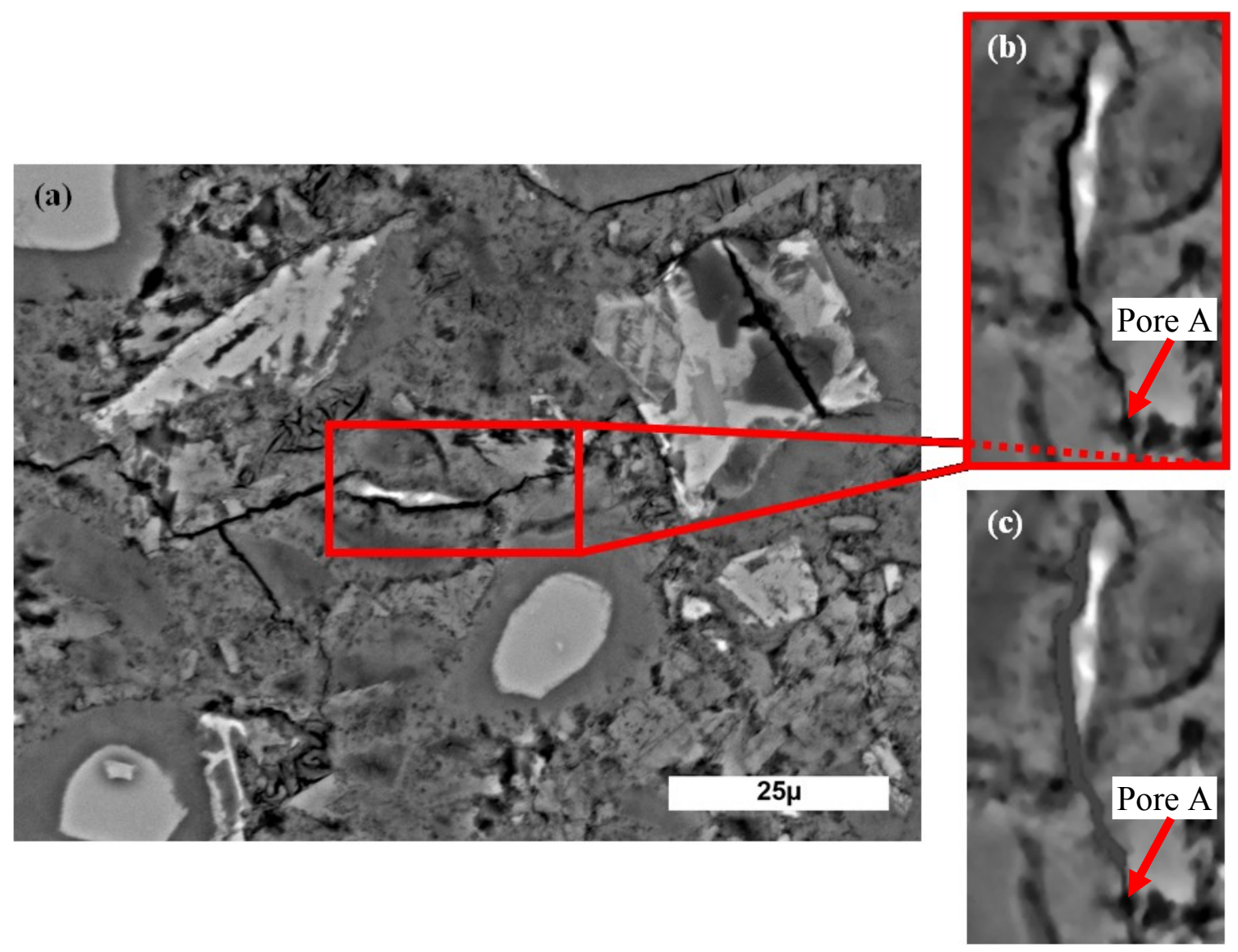

Figure 4-5: A SEM image of a freeze-thawed cement paste specimen with a $\mathrm{w} / \mathrm{c}=$ 0.47. (a) Cracks developed in the cement paste microstructure due to crystallization pressure, and an interested area (as shown with red box) was selected for CZM simulation and validation. (b) The selected image section is cropped, resized, filtered, and rotated to develop digital sample for CZM simulation. (c) The processed image was edited to patch the crack with matrix phase for fracture model simulation. 


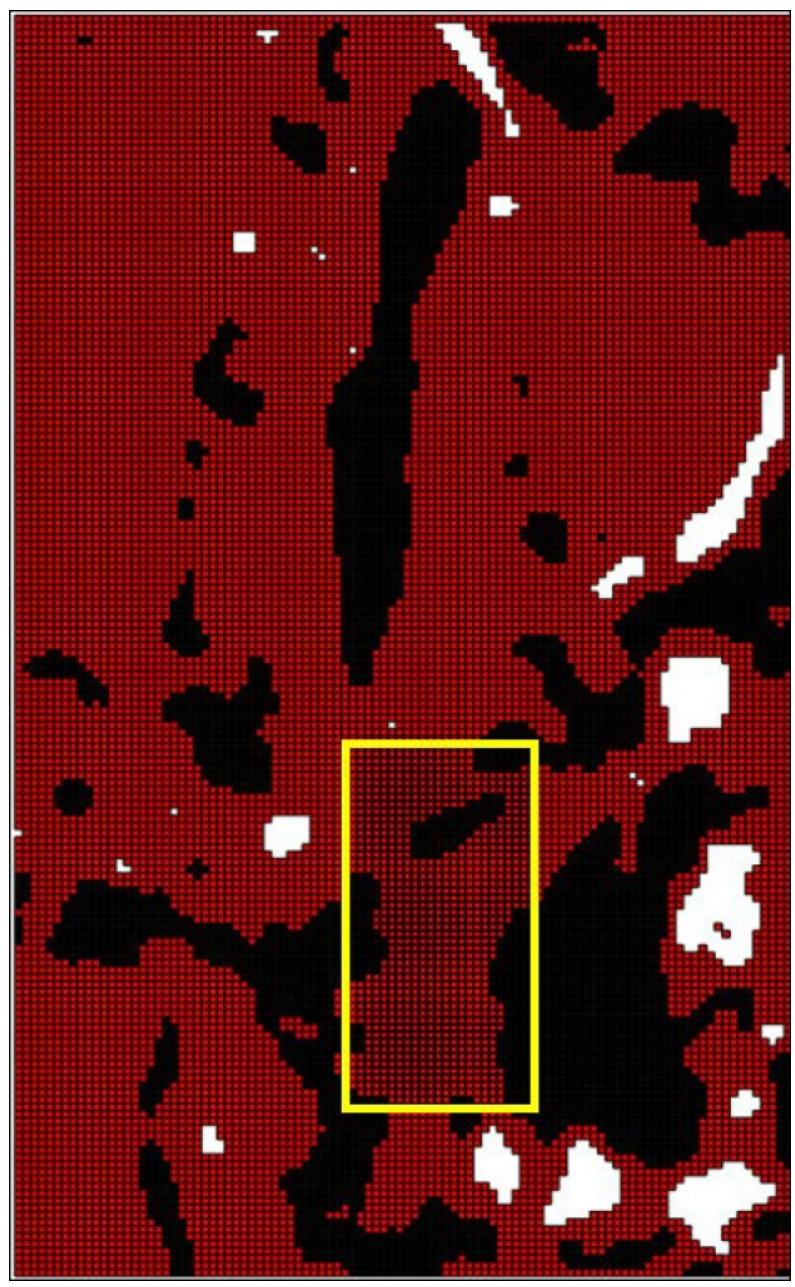

Figure 4-6: The constructed FE model based on the digital SEM image on damaged cement paste sample.

In constructing the mesh, a 4-node bilinear element or CPS4 was employed. Each element was equilent to the size of a pixel which has a dimension $0.096 \times 0.096 \mu \mathrm{m}^{2}$. Multiple columns of CZM elements were defined on top of the pore A, as highlighted in Figure 4-6. Similar CZM properties from the CT test model were defined based on the material interface. In boundary conditions, roller supports were defined around the edges of the model. 


\subsubsection{3-D Image Reconstruction on SEM Samples}

An undamaged cement paste SEM image (Figure 4-7(a)) was utilized to perform the 3-D image reconstruction. The cement paste sample was undamaged and has a w/c $=$ 0.47. The image size of the SEM image is $300 \times 300$ pixels. A threshold of 40 in magnitude was applied by using ImageJ to differentiate between the pore and solid phases. That is, the pore phase is black in color (0) and solid is white in color (255), as shown in Figure 4-7 (b). To perform the 3-D image reconstruction program (ftp://ftp.nist.gov/pub/bfrl/bentz/permsolver) developed by Mr. Dale Bentz, binary image data was generated with the pore and solid phases were assigned with 0 and 1 , respectively.

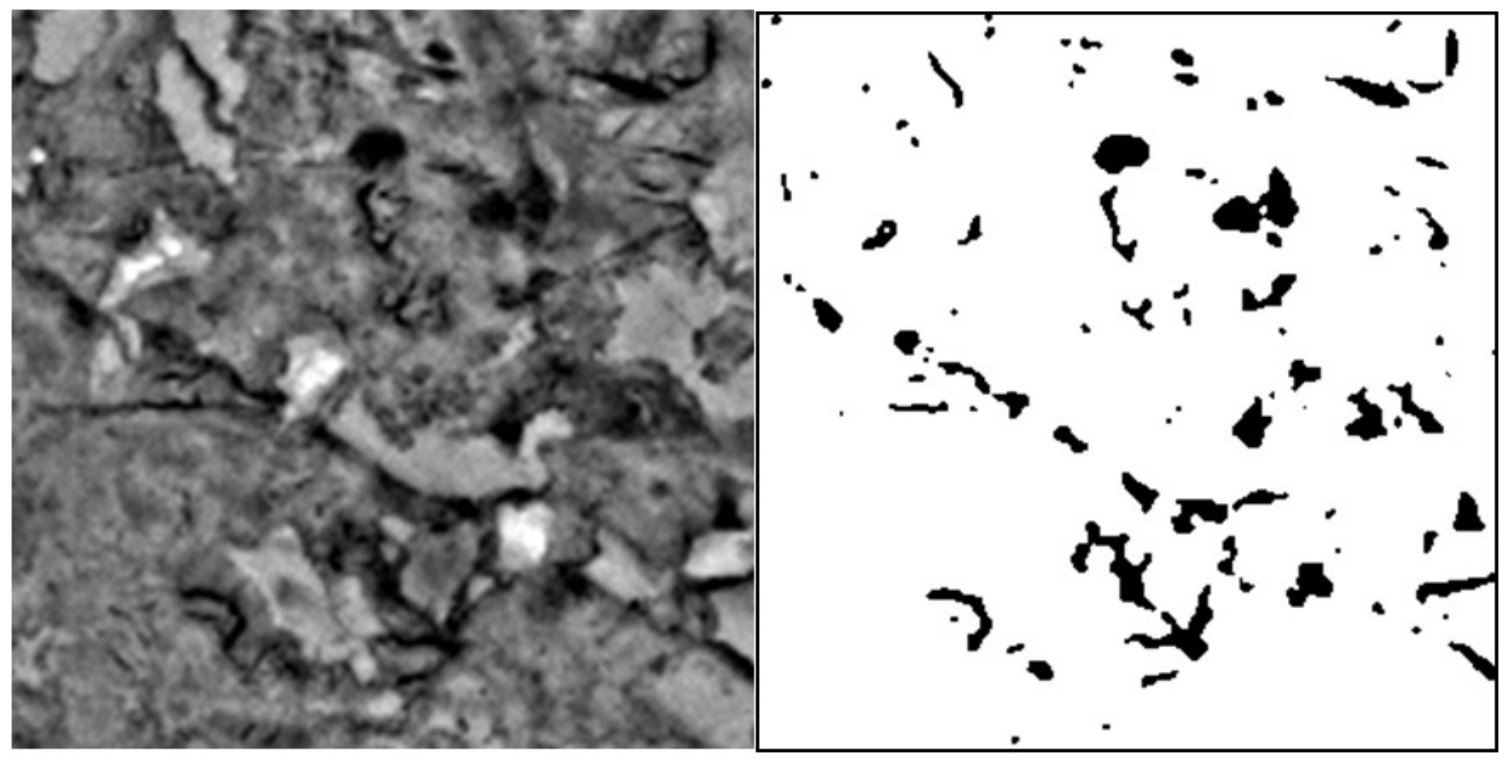

Figure 4-7: Image processing on SEM image of an undamaged cement paste sample with $w / c=0.47$. (a) An SEM image with dimension of $300 \times 300$ pixels. (b) Image processing on SEM image after threshold of 40 in magnitude was applied. 
The image data was then saved in the correlation program folders to compute the 2-D correlation function in $S(x, y)$ form for the SEM image. Equation (4-1) shows the $S(x, y)$ form:

$$
S(x, y)=\sum_{i=1}^{M} \sum_{j=1}^{N} \frac{I(i, j) \times I(i+x, y+j)}{M \times N}
$$

where $M$ and $N$ refer to the total pixel numbers in the rows and columns, whereas, $i$ and $j$ refer to the indices of pixels in the rows and columns. For this image, both $M$ and $N$ are equal to $300 . I(i, j)$ is a discrete valued function which equals to one and zero for solid and pore pixels, respectively (Bentz and Martys 1994). $I(i+x, y+j)$ is a function that represent the periodic boundaries are applied when $(i+x, y+j)$ exceeds the boundaries of a 2-D image. The $x$ and $y$ values in the function $S(x, y)$ range from 0 to 60 . The function $S(x, y)$ is then transformed to $S(r)$ in polar form for distances $r$ in pixels and the equation is given as:

$$
S(r)=\frac{1}{2 r+1} \sum_{l=0}^{2 r} S\left(r, \frac{\pi l}{4 r}\right)
$$

where $S(r)$ is equivalent to $S(r \cos \theta, r \sin \theta)$, which is determined from the bilinear interpolation from the function $S(x, y)$. From this correlation function, it is determined that the porosity of the SEM image is approximately $6.35 \%$.

After executing the correlation programs, rand3d.c was executed to construct 3-D image data based on the generated correlation function data. A threshold value of $6.35 \%$ was applied to construct the 3-D image. Figure 4-8 (a) demonstrates a random 3-D image reconstruction slice that was generated from the rand3d.c program. Sintering algorithm 
was then performed for the purpose of noise reduction. As shown in Figure 4-8(b), the 3D image slice is noise free after performing sintering algorithm with a hydraulic radius of 5. The sintered image data were then generated with 300 2-D image slices. These data were readable by using ImageJ and each individual image slices were stacked to construct 3-D image, as shown in Figure 4-9(a). The image size has a dimension of $300 \times$ $300 \times 300$ pixels. To visualize the pore structure in the reconstructed 3 -D image, a pore structure 3-D image was generated in ImageJ, as shown in Figure 4-9(b). Figure 4-10 illustrates the flow of 3-D image reconstruction on the SEM image of the undamaged cement paste sample with $\mathrm{w} / \mathrm{c}=0.47$.

(a)

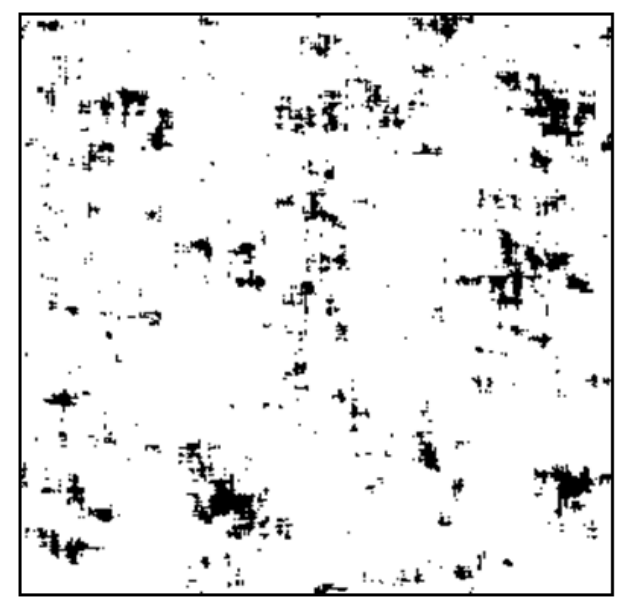

(b)

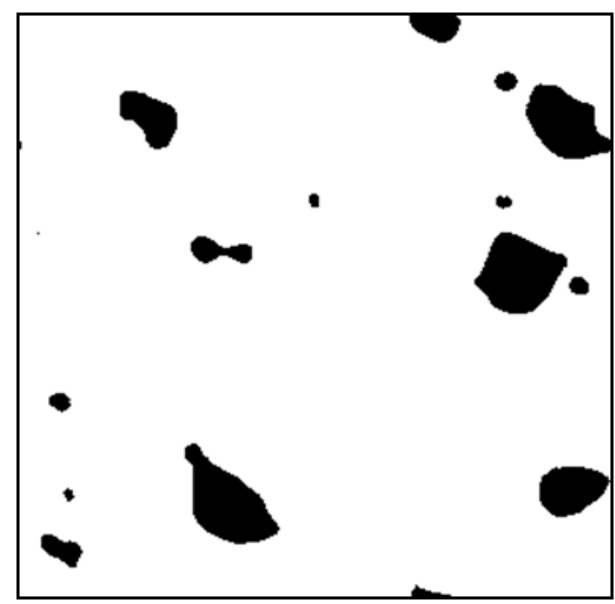

Figure 4-8: The 3-D image reconstruction technique was applied on the SEM image of an undamaged cement paste sample with w/c $=0.47$ : (a) A random 3-D image slice was generated with a porosity of $6.35 \%$. (b) A 3-D image slice after executing sintering algorithm to remove noises in the image. 


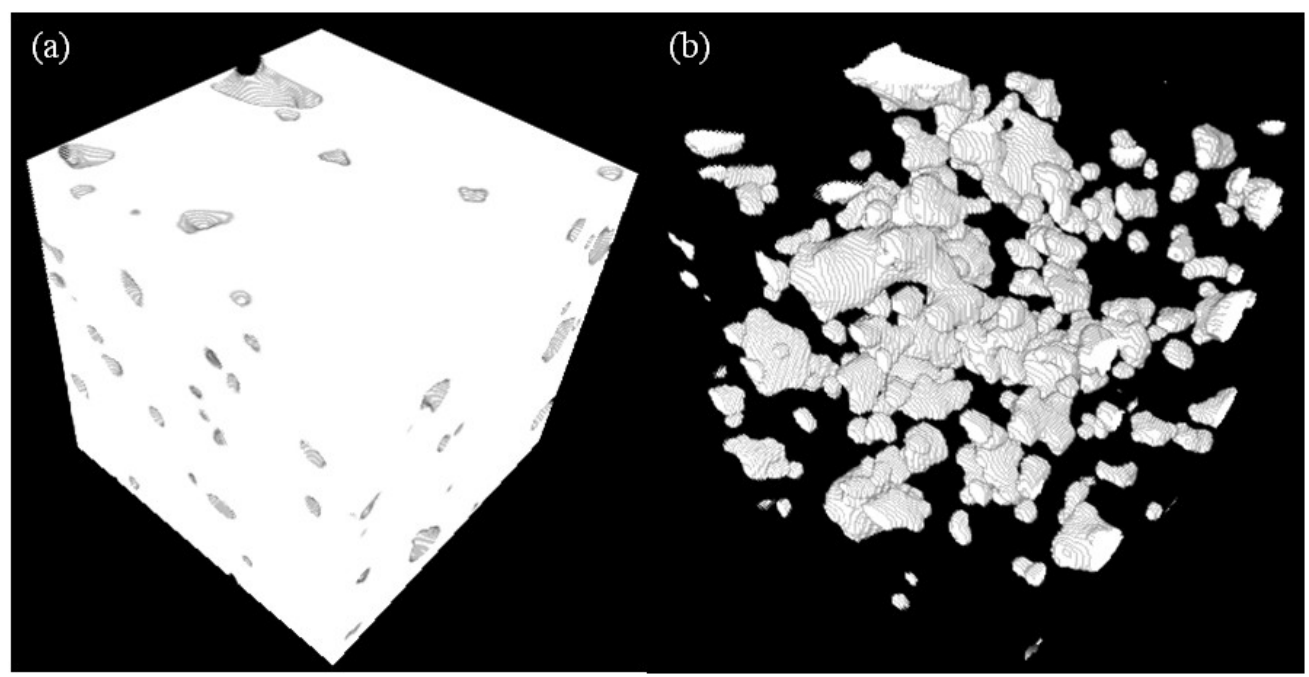

Figure 4-9: 3-D image reconstruction generation on SEM image of an undamaged cement paste sample with $\mathrm{w} / \mathrm{c}=0.47$ : (a) A 3-D reconstructed image after stacking 300 image slices. (b) A 3-D view of pore structure for the reconstructed 3-D image. 
SEM image on a cement paste sample, a section of $300 \times$ $300 \times 300$ pixels was selected for $3 \mathrm{D}$ image reconstruction
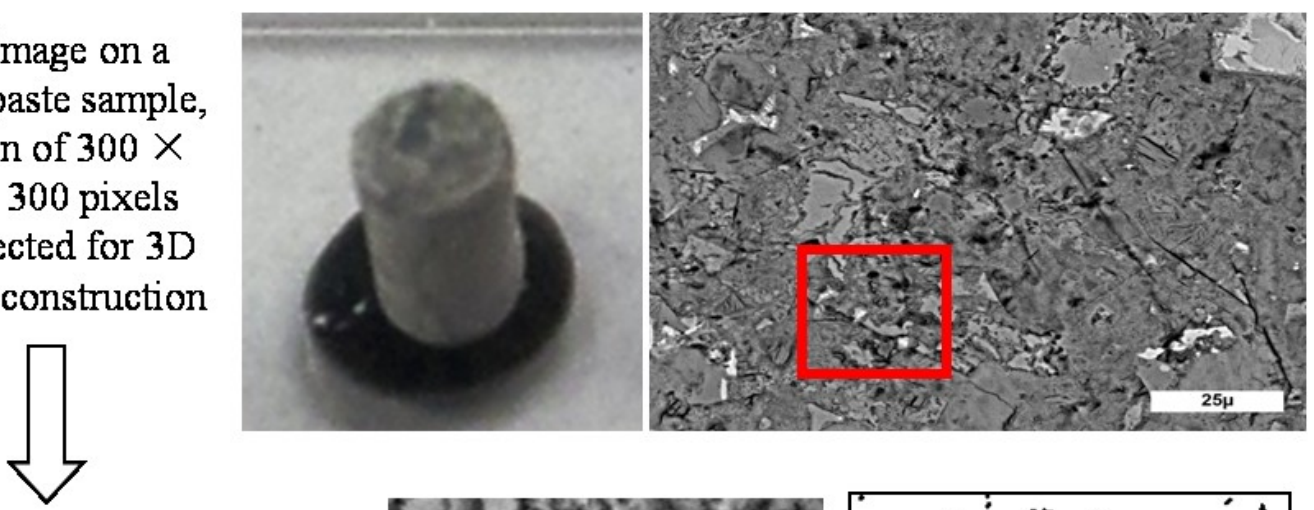

The image slice was processed to obtain 2 phase data

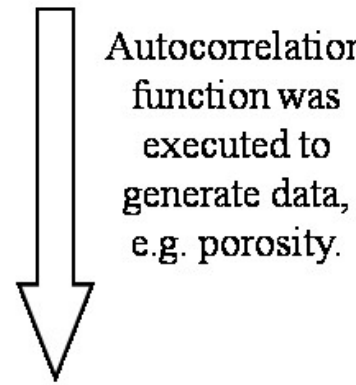

A 3D image was reconstructed by using rand3d.c. The image was filtered to remove noises through sintering algorithm.

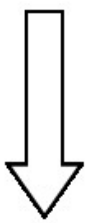

The generated 3D image slices were stacked to build 3D image. A pore structure was constructed too.
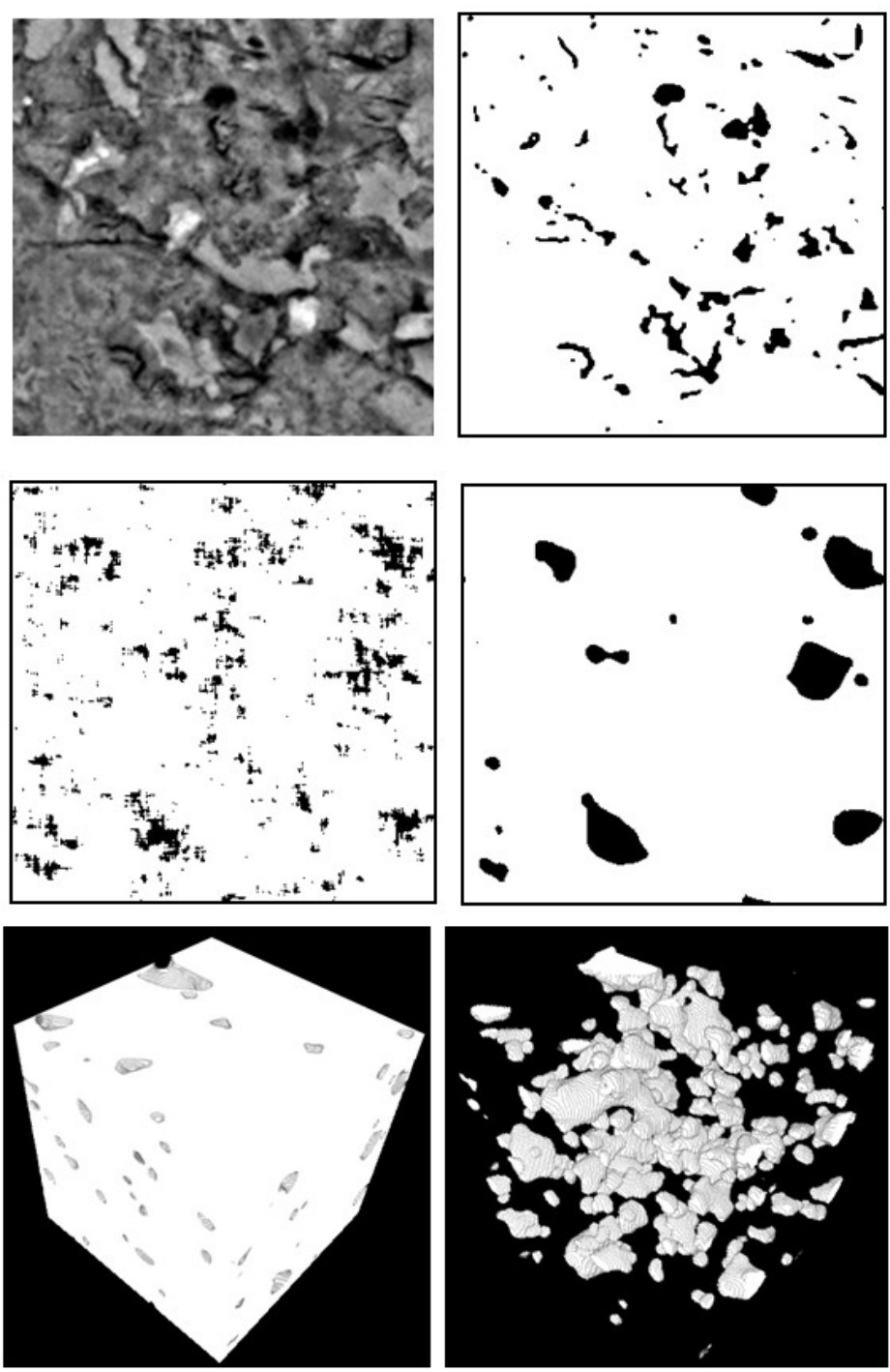

Figure 4-10: Schematic highlights the steps on 3-D image reconstruction from an SEM undamaged cement paste sample with $\mathrm{w} / \mathrm{c}=0.47$. 


\subsection{TXM Sample Image Processing}

The original Transmission X-ray Microscopy (TXM) images were captured and read using AmiraMesh program. The program is not available at MTU, so only ImageJ can be utilized to read the image data. However, in order to open the image data, several plug-ins have to be installed into the ImageJ program folder. The available AmiraMesh plug-in for ImageJ can be found in the Virtual Insect Brain Lab website (www.neurofly.de). Among the installed plug-ins are Amira Surface Viewer and AmiraMesh Reader/Writer. The Amira Surface Viewer will allow the user to visualize the surfaces created by Amira, whereas AmiraMesh Reader/Writer will allow the user to read and write generated 3-D stacks in the Amira Mesh format. Upon installing the plugins, the additional AmiraMesh plug-ins in the Plugins option in ImageJ can be seen.

As the TXM image file was opened, some image processing steps were performed to see clearly the detail microstructure of cement paste clearly. As shown in Figure 4-11(a), the TXM image of the cement paste microstructure was not clear. The cement paste has a w/c $=0.45$ and had performed freeze test for 60 cycles. In order to view the microstructure clearly, the color balance technique was applied in ImageJ. Figure 4-11(b) shows the result after adjusting the color balance with a dark black ground and enabled the cement paste microstructure to be seen clearly. The intensity magnitude differentiates the phases in the microstructure based on the density of the materials. That is, the unhydrated cement, hydrated cement, and pores are shown in white, gray, and black colors, respectively. 


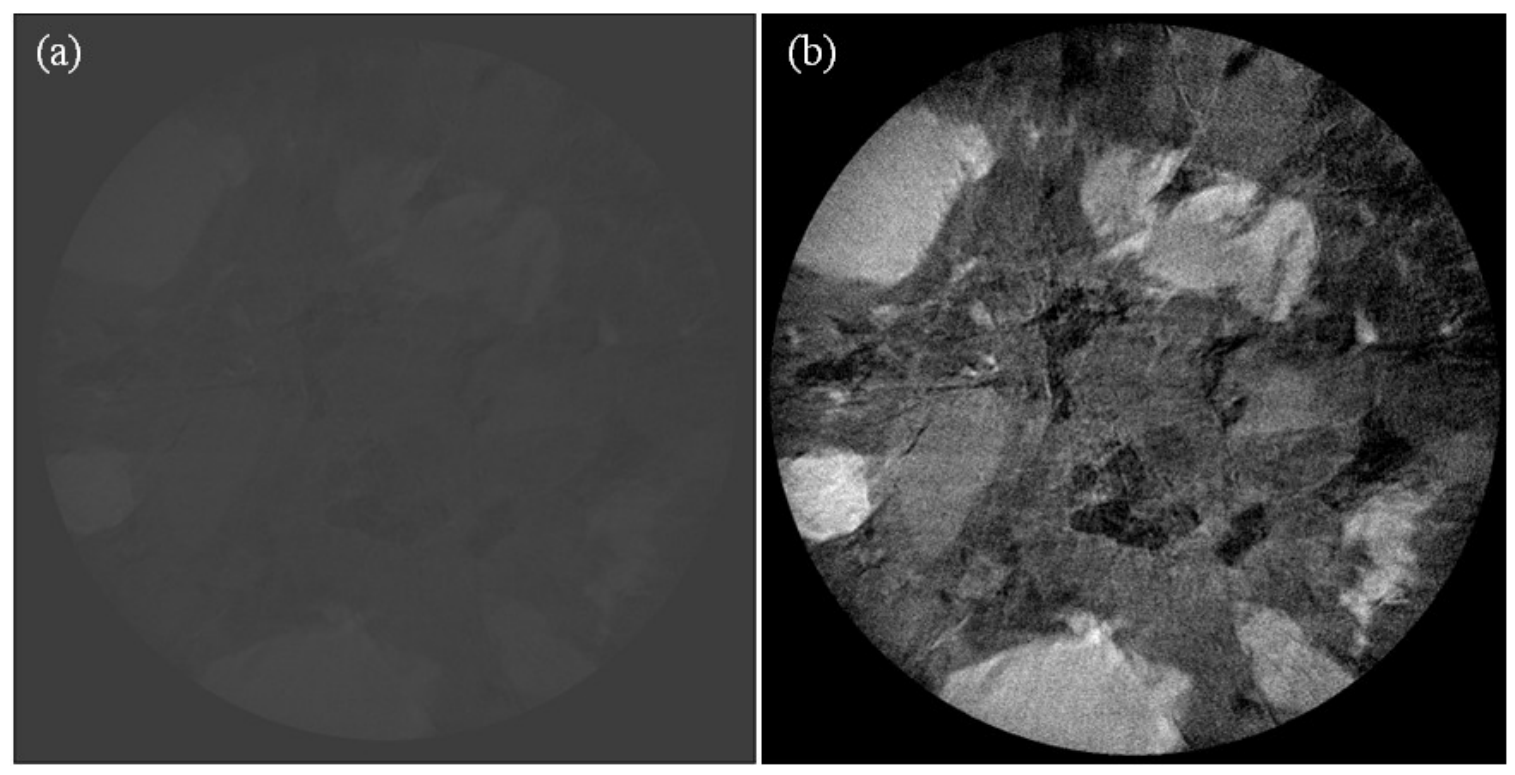

Figure 4-11: The digital image of a freeze-thawed cement paste microstructure acquired from the TXM (a) before and (b) after image processing.

In order to view 3-D cement paste microstructure images, ImageJ 3-D Viewer in the Plugins was applied to stack the 2-D digital TXM image slices to generate a 3-D image, as shown in Figure 4-12. In order to reduce noise in the TXM images, the filtering technique was applied by using the median filtering technique. A radius of 2.0 pixels was applied for the median filtering. Figure 4-13 shows the output of the filtered image. 


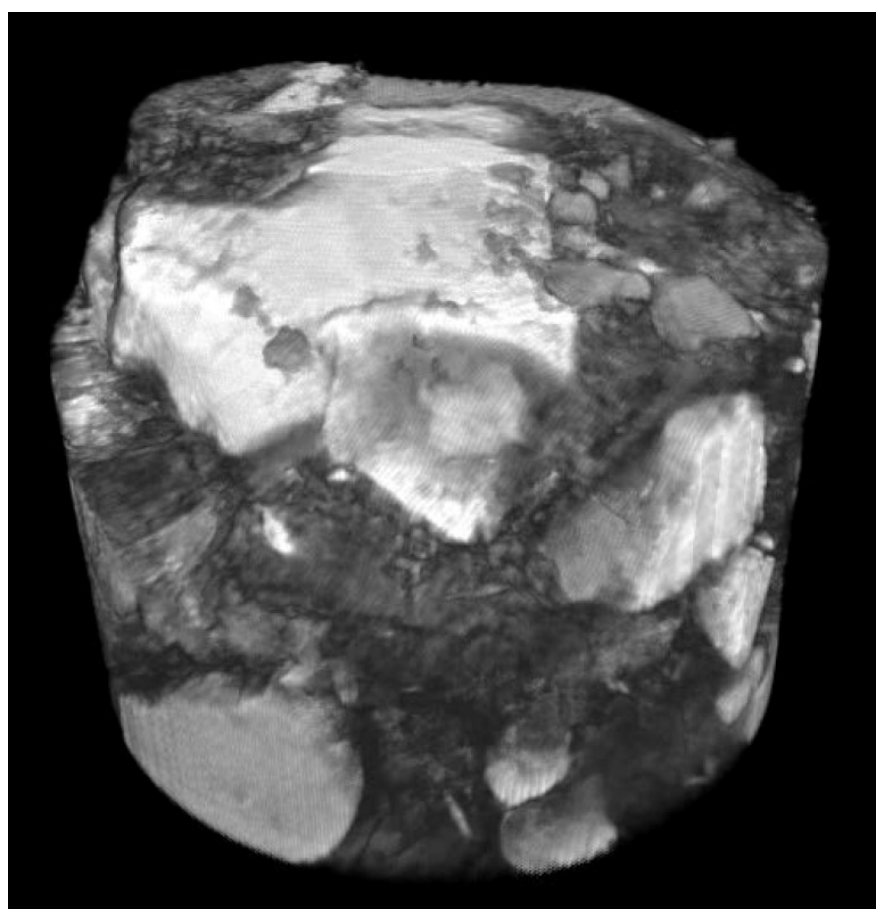

Figure 4-12: A 3-D image was constructed from the 2-D TXM digital image slices using the ImageJ 3-D Viewer technique.

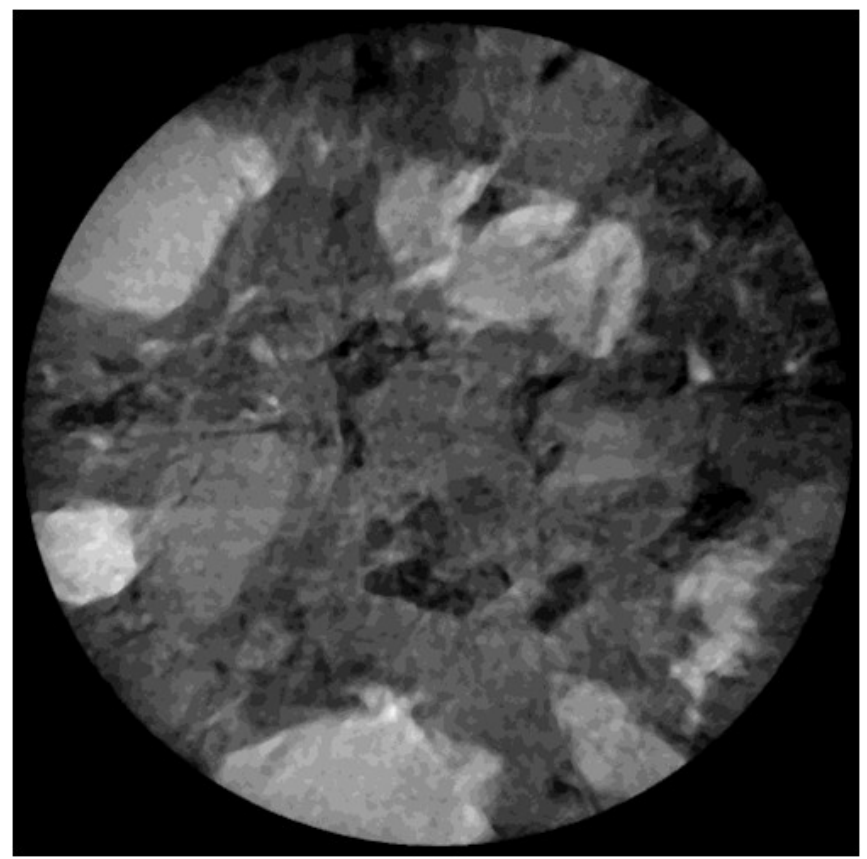

Figure 4-13: The cement paste microstructure TXM image after filtered using median filtering technique. 
In order to utilize some computational modeling programs, such as PERMSOLVER, CHEMHY3D, or HYDRA3D, which are developed by the National Institute of Standards and Technology (NIST), a specific 3-D image size was selected. For this dissertation study, a 3-D image size of $300 \times 300 \times 200$ pixels was selected. Figure 4-14 illustrates the Specify option was applied to select the area of interest to be cropped, with an area of $300 \times 300$ pixels. There are some images that are filled with empty spaces, especially those images closer to the gold. Thus, the stacked TXM images were separated into individual image files to select interested images. For the cement paste TXM images, only the first 200 images were restacked while the remaining images were removed as a result of empty spaces rather than pores that existed in the remaining images. Figure 4-15 shows the result after image cropping and restacking were performed. 


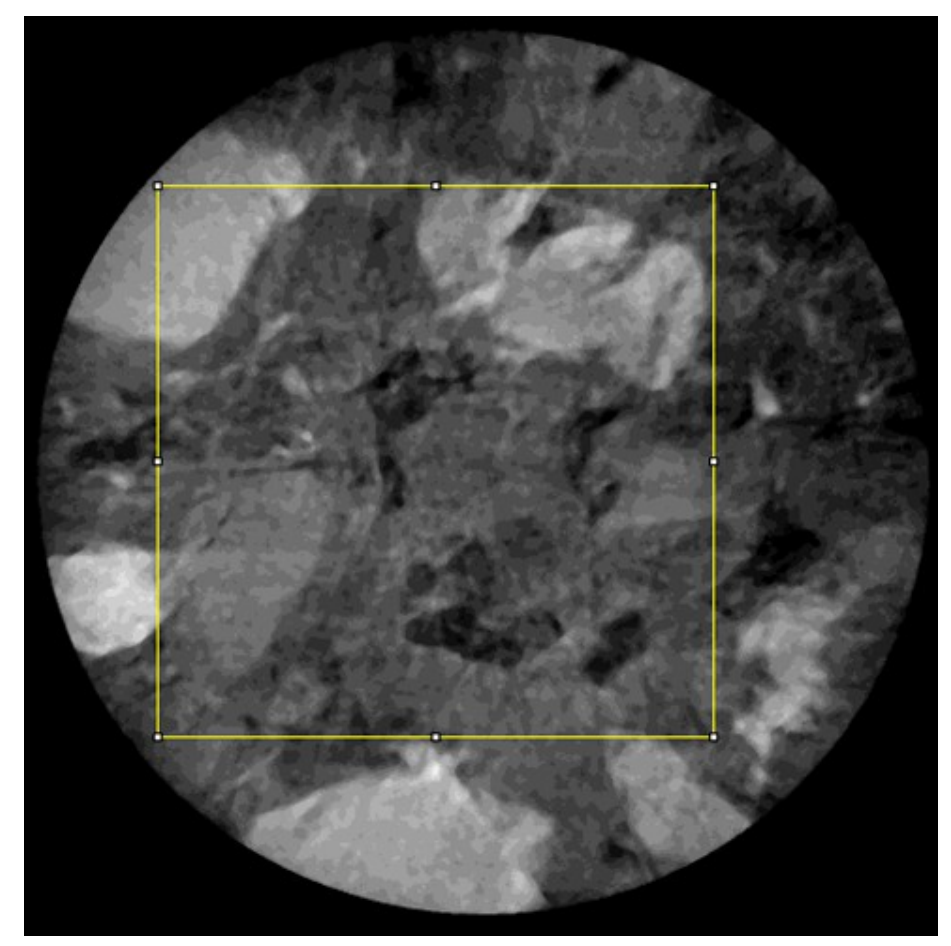

Figure 4-14: A cross section of $300 \times 300$ pixels was selected for cropping through Specify option in ImageJ (http://rsb.info.nih.gov/ij/).

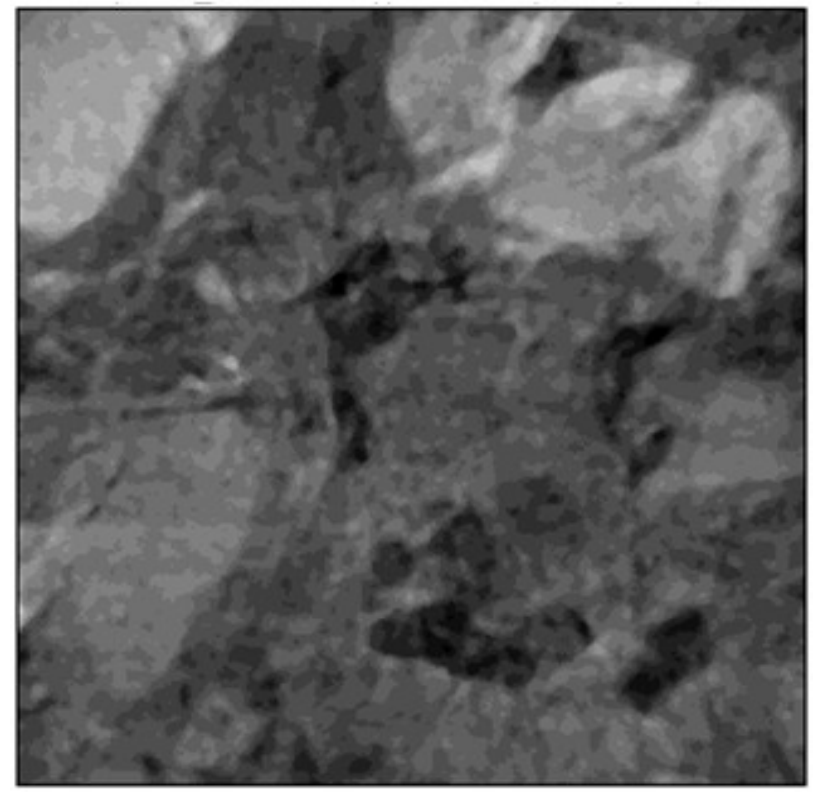

Figure 4-15: The result of re-stacked on cropped cement paste microstructure TXM image with image size of $300 \times 300 \times 200$ pixels. 
To display only pore structures in 3-D view, a threshold was applied to identify the pores in the image from the solids. As shown in Figure 4-16, a threshold magnitude of 62 was selected as apparent pores were displayed. By applying ImageJ 3-D Viewer, a 3D image size of $300 \times 300 \times 200$ pixels was constructed with only pore structures displayed, as shown in Figure 4-17.

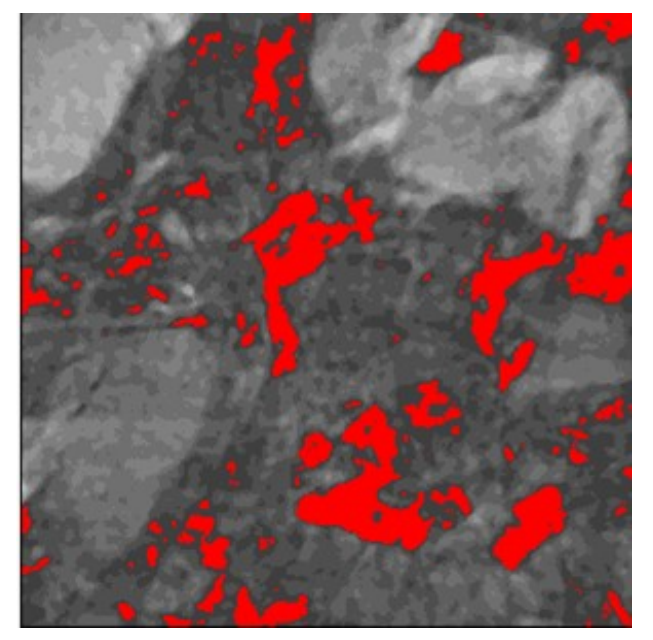

Figure 4-16: A threshold of 62 in magnitude was applied as it demonstrates apparent pore structure in the ImageJ (http://rsb.info.nih.gov/ij/). 


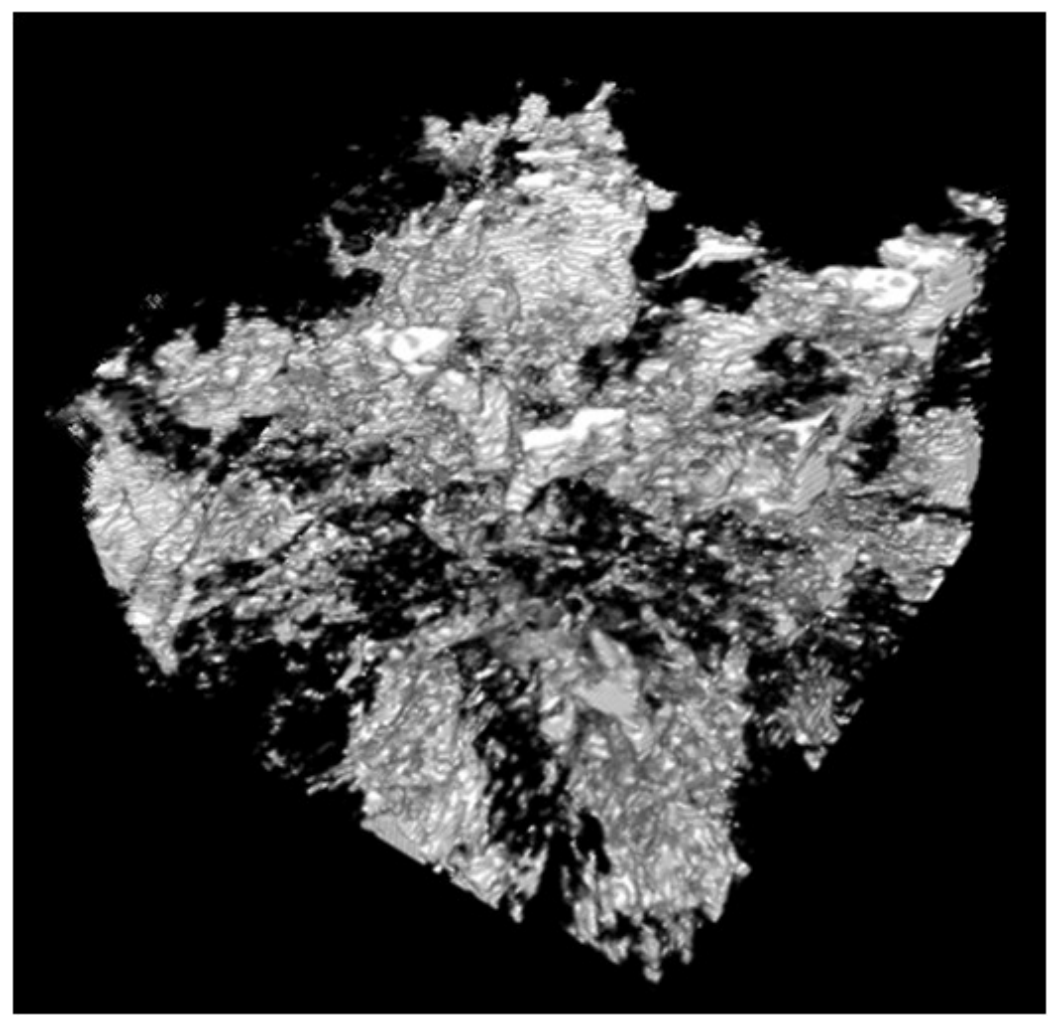

Figure 4-17: A $3 D$ view of $300 \times 300 \times 200$ pixels of cement paste microstructure TXM image that shows only the pore structures.

\subsection{Concluding Remarks:}

All the images were successfully processed by maintaining the original details of the images, not only in macro scales but also in micro and nano scales. In macro scale, a concrete beam from the SEB bending test was conducted with image processing. The concrete beam was scanned with high resolution scanner and was edited to remove noise and differentiate the phases. In order to be read in the XFEM program, the elliptical fitting technique was employed to generate elliptical inclusions, which represent the aggregates in the actual concrete beam. On the other hand, the actual aggregate shapes 
were constructed in the bilinear CZM model. Only a portion of the concrete beam image was utilized to perform fracture simulation in order to reduce the computational time.

In micro scale, an undamaged and a damaged cement paste sample as a result of freeze-thaw test were utilized to perform fracture simulations. All the images were processed to remove noise and the shapes of unhydrated cement particles and pores were maintained as in the actual image. Only the bilinear CZM technique was employed. For the undamaged cement paste sample, a section of image with multiple pores was used to construct a CT test model in ABAQUS. The section was selected as the pore structures had the potential to occur coalescing when fracture occurred. The CT test model was utilized to observe the capability of the bilinear CZM technique on simulating fracture behavior on a porous cement paste sample. For the damaged cement paste sample, a damaged region, which consists of crack developed with coalescing along pore structures, was selected as the chances of the facture due to crystallization pore pressure is high. The crack line was patched with hydrated cement phase and the bilinear cohesive elements were defined on the patched zone. This approach was done in order to validate the internal frost damage simulation to the actual damaged cement paste.

The 3-D image reconstruction technique was carried out to generate a 3-D image from the 2-D SEM image through the correlation function and sintering algorithm. The microstructure of the reconstructed 3-D image is different from the original 2-D image and is periodic while maintaining the physical properties of a material, i.e. porosity. In this study, an undamaged cement paste sample with $\mathrm{w} / \mathrm{c}=0.47$ and porosity of $6.35 \%$ was utilized for 3-D image reconstruction. The generated 3-D image slices can be utilized to perform fracture simulation of the internal frost damage with known hydraulic radius. 
The acquired TXM 3-D image from a damaged cement paste sample with $\mathrm{w} / \mathrm{c}=$ 0.45 was processed by using ImageJ with proper plug-ins. A 3-D image was constructed after stacking all the image slices. In order to view the pore structure of the sample, a 300 $\times 300$ pixels section was selected and processed with median filter of 2.0 in radius for noise reduction. With a threshold magnitude that represented the pores defined, a 3-D pore structure was constructed. The successful image generation from the TXM image allowed the internal frost damage analysis in nano-pore system to be carried out. 


\section{CHAPTER 5 INTERNAL FROST DAMAGE COMPUTATION WITH IDEALIZED PORES UNDER CRYSTALLIZATION PRESSURE}

\subsection{Overview}

This study investigated the internal frost damage due to ice crystallization pressure in the concrete pore system. The thermodynamic analysis and micro-damage models were developed to study the internal frost damage in concrete samples. In the micro-scale pore system, the crystallization pore pressure at sub-cooling temperature was calculated using interface energy balance with thermodynamics analysis. The largest crystallization pore pressure on the pore wall was input for the fracture simulation with the developed extended finite element method (XFEM) in MATLAB and bilinear cohesive zone modeling (CZM) in ABAQUS. Two scenarios of internal frost damage analyses were performed by using the XFEM technique. The simulation results had shown that the crystallization pore pressure at certain temperature and pore radius can cause damages to porous concrete. The bilinear CZM was employed to overcome the limitation of XFEM to simulate coalescing in a porous system. The bilinear CZM simulation results had shown the capability of the technique to model fracture behavior in 2-D and 3-D through CT test and SEB bending test models. 


\subsection{Internal Frost Damage Analysis within Idealized Pores of 2-D}

\section{Samples under Sub-cooling Temperatures}

The crystallization pore pressure analysis and XFEM fracture simulation were conducted to demonstrate the internal frost damage processes in two scenarios in idealized pore structures. In the first scenario, the internal frost damage process was analyzed within a pore with connected capillary pores. Whereas in the second scenario, the internal frost damage in a multi-pores system with connecting capillary pores was analyzed. Both scenarios were performed by using the XFEM technique with tensile pressure loadings applied at the fracture point. The maximum normal stress criterion $\sigma_{N}$ (Dowling 2007) was set in the XFEM program as the fracture criteria for the internal frost damage analysis on cement paste models. The maximum normal stress criterion is given as follows:

$$
\begin{gathered}
\sigma_{N}=\mathbf{M A X}\left(\left|\sigma_{1}\right|,\left|\sigma_{2}\right|\right) \\
\sigma_{1,2}=\frac{\sigma_{x x}+\sigma_{y y}}{2} \pm \sqrt{\left(\frac{\sigma_{x x}+\sigma_{y y}}{2}\right)^{2}+\tau_{x y}^{2}}
\end{gathered}
$$

where the MAX denotes the highest stress magnitude selected between $\sigma_{1}$ and $\sigma_{2}, \sigma_{1,2}$ are the principal stresses, $\sigma_{x x}$ and $\sigma_{y y}$ are normal stresses in $\mathrm{x}$ - and y-directions, and $\tau_{x y}$ is the shear stress. The crack will develop when the generated stresses on the crack tip were greater than or equivalent to the maximum normal stress. 


\subsubsection{Scenario 1: Single pore system}

As shown in Figure 5-1, one spherical pore with a radius $r_{s}=50 \mathrm{~nm}$ is connected by cylindrical capillary pores with a radius of $10 \mathrm{~nm}$. The Gibbs-Thomson's formula in Equation (1-1) was applied to determine the freezing temperature for the spherical and cylindrical pores. Pore curvature was determined by the differentiation of pore area with respect to pore volume, which is given as follows:

$$
\kappa_{C L}=\frac{d A}{d V}
$$

where $A$ and $V$ are the area and volume, respectively. Thus, for a spherical pore with $A=$ $4 \pi r^{2}$ and $V=4 \pi r^{3} / 3$, the curvature of the pore is: $\kappa_{C L}=2 /\left(r_{p}-\delta_{W}\right)$. Similarly, for a cylindrical pore with $A=4 \pi r^{2} L$ and $V=\pi r^{2} L$, where $L$ is the length of the cylinder, the curvature of the pore is: $\kappa_{C L}=1 /\left(r_{p}-\delta_{W}\right)$. By substituting the determined pore curvature value into Equation (1-1), the freezing temperature for the spherical pore without ice bulged into the capillary pore channel was found to be $-1.39^{\circ} \mathrm{C}$. As shown in Figure 5-1 (a), the ice had started to bulge into the capillary pore channel with a radius of $10 \mathrm{~nm}$, which indicated that the larger pore and the capillary pores were having the same freezing temperature. That is, the freezing temperature in the pore system was now found to be $-7.69^{\circ} \mathrm{C}$. 


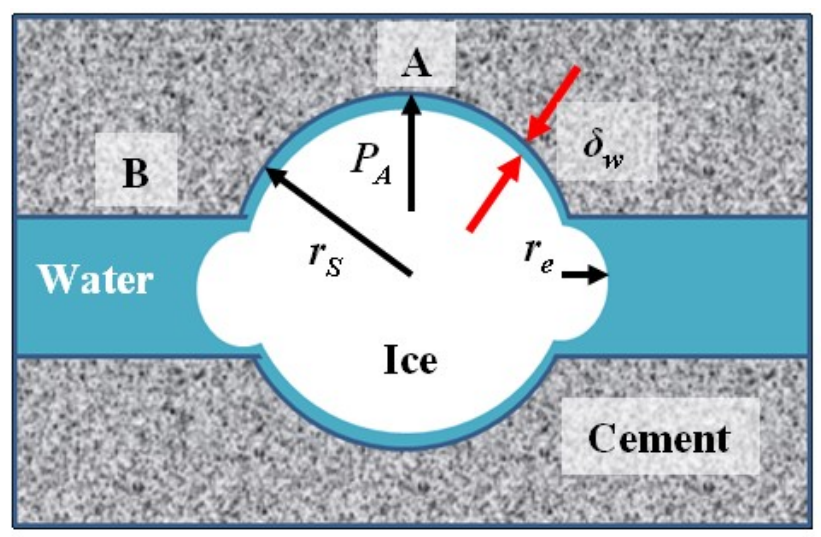

(a)

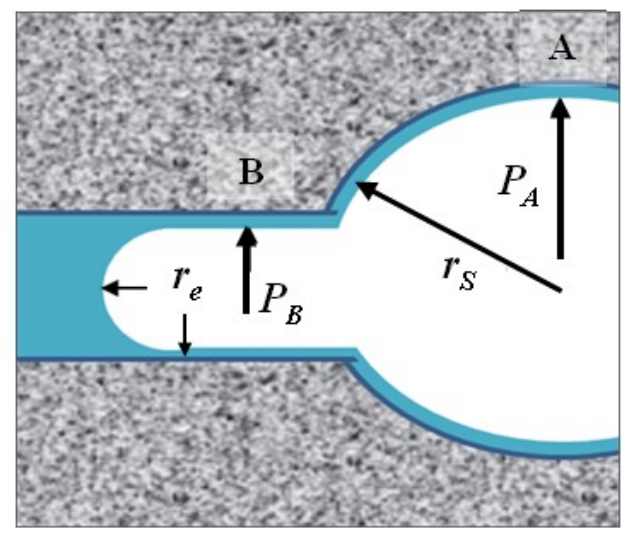

(b)

Figure 5-1: Schematic illustration of crystallization pressure effect within a pore system in the cement paste (a) before reaching the threshold temperature $\left(-7.69^{\circ} \mathrm{C}\right)$, with a pressure $P_{A}=7.52 \mathrm{MPa}$. (b) As the ice at free ends grows into capillary pore after reaching the threshold temperature with pressure $P_{B}=4.62 \mathrm{MPa}$ based on GibbsThomson formula.

By assuming that the capillary pores were in saturated condition, the curvature at the bulged ends turned into hemispherical shape. According to Gibbs-Thomson in Equation (1-1), an ice can bulge into cylindrical pore with a radius $r_{p}$ which can be determined by this following equation.

$$
r_{p} \geq \delta_{w}+\frac{2 \gamma_{C L}}{\left(T_{m}-T\right) \Delta S_{f v}}
$$

where $\delta_{w}$ is the thin water layer thickness, which is approximately $0.9 \mathrm{~nm}$ and $T$ is the applied sub-cooling temperature.

In order to determine the generated crystallization pore pressure, Equation (1-4) was employed. Thus, for the spherical pore in Figure 5-1(a), Equation (1-4) becomes $P_{A}=\gamma_{C L}\left(2 /\left(r_{e}-\delta_{w}\right)-2 /\left(r_{s}-\delta_{w}\right)\right)$ and the generated maximum crystallization pore pressure was determined to be $7.52 \mathrm{MPa}$. As shown in Figure 5-1(b), when the sub- 
cooling temperature further reduces slightly below $-7.69{ }^{\circ} \mathrm{C}$, the bulged ice began to penetrate into the cylindrical capillary pore. The crystallization pore pressure generated along the cylindrical wall (at Point B) can be calculated by assuming the free end remains in hemispherical shape with a radius of $10 \mathrm{~nm}$. Hence, the confining pressure on the cylindrical capillary wall (Point B) was found to be $4.62 \mathrm{MPa}$ with the equation $P_{B}=\gamma_{C L}\left(2 /\left(r_{e}-\delta_{w}\right)-1 /\left(r_{e}-\delta_{w}\right)\right)$. Both pressures at locations A and B exceeded the tensile strength of the cement paste theoretically, which is approximately $2 \mathrm{MPa}$.

In order to validate the fracture theory, the computed pressure was input into the developed XFEM program to simulate the crack propagation within a uniform cement paste sample. The mesh of the model was constructed with four-node bilinear square elements and each element has a dimension of $1 \mathrm{~nm} \times 1 \mathrm{~nm}$. Only half of the pore structure was modeled to reduce the computation time. Hence, a hemisphere with a radius of $50 \mathrm{~nm}$ for the spherical pore model and a hemisphere with a radius of $15 \mathrm{~nm}$ for the capillary pore channels model were constructed. Roller supports were applied around the boundaries of the cement paste model to create a confined boundary. The Young's modulus of $22 \mathrm{MPa}$ and Poisson's ratio of 0.25 were defined for the material properties of the cement paste model. A tensile strength of $2 \mathrm{MPa}$ was defined as the threshold of the maximum principal stress failure criteria. An initial crack length of 2 elements or 2 $\mathrm{nm}$ were defined at the center of the pore, that is, Points A and B (assuming as the weakest spots) of the spherical pore (Figure 5-2 (a)) and capillary pore channel (Figure 5-2 (b)), respectively. Tensile pressures loadings were applied on the elements that defined the initial crack with magnitudes of $7.52 \mathrm{MPa}$ and 4.62 MPa at Points A and B, respectively. A propagation length of 2 elements in each iteration will be generated when 
the computed stresses exceeded the tensile strength of the cement paste, based on the maximum normal stress criterion (Equations (5-1) and (5-2)).

Figure 5-2 demonstrates the XFEM fracture simulation result on the spherical pore and capillary pore channel due to crystallization pressure effect. As shown in Figure 5-2 (a), the induced tensile pressure loading at Point A with a magnitude of $7.52 \mathrm{MPa}$ was sufficient to cause the crack to propagate. This is because of the maximum principal stress generated was higher than the predefined tensile strength $\left(\sigma_{N}=2 \mathrm{MPa}\right)$. Similarly in Figure 5-2 (b), the hoop stress generated at Point B along the surface perpendicular to the pore cross section exceeded the tensile strength due to an applied tensile pressure loading of 4.62 MPa. The crack extended by four elements in both cases as a result of exceeding the threshold of the failure criteria. The results have validated that the theoretical thermodynamic analysis can correctly predict the induced pressure in the porous cement paste. 
(a)

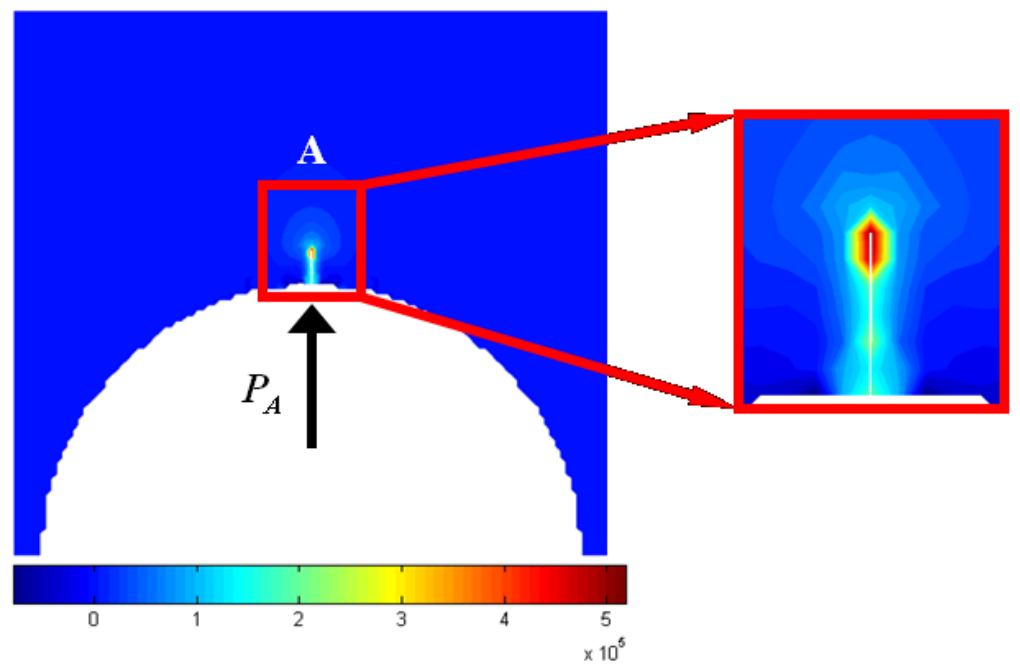

(b)

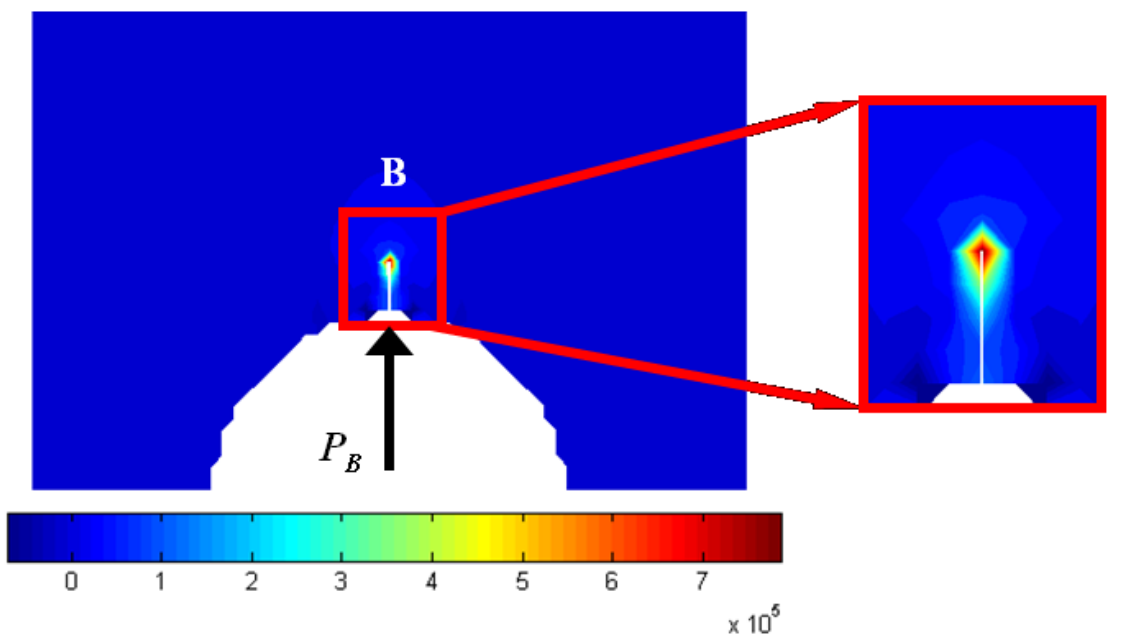

Figure 5-2: XFEM simulation of pore system as the threshold temperature $\left(-4.85^{\circ} \mathrm{C}\right)$ had reached and ice grew into capillary pore. (a) The principal stress $\sigma_{1}$ on a pore with radius of $50 \mathrm{~nm}$ and $P_{A}=4.15 \mathrm{MPa}$. (b) The principal stress $\sigma_{1}$ contour generated on the $15 \mathrm{~nm}$ channel with $P_{B}=2.9 \mathrm{MPa}$.

\subsubsection{Scenario 2: Multiple Pore System}

In the second damage scenario, two spherical pores were connected with cylindrical channels. The pore geometries are as listed in Figure 5-3(a), which shows two spherical pores with radii of $50 \mathrm{~nm}$ and $30 \mathrm{~nm}$ for Pore 1 and Pore 2, respectively. The figure also shows two capillary pore channels with radii of $20 \mathrm{~nm}$ and $10 \mathrm{~nm}$ for pore 
channel 1 and channel 2, respectively. By using the Gibbs-Thomson equation (Equation (1-1)), the freezing temperature for the pores and capillary pore channels in the pore system were determined, as highlighted in Table 5-1.

Table 5-1: Freezing temperatures in the pore system for scenario 2.

\begin{tabular}{|c|c|c|}
\hline Location & Radius & Freezing Temperature \\
\hline Pore 1 & $50 \mathrm{~nm}$ & $-1.29^{\circ} \mathrm{C}$ \\
\hline Pore 2 & $30 \mathrm{~nm}$ & $-2.34^{\circ} \mathrm{C}$ \\
\hline Capillary Pore Channel 1 & $20 \mathrm{~nm}$ & $-3.58^{\circ} \mathrm{C}$ \\
\hline Capillary Pore Channel 2 & $10 \mathrm{~nm}$ & $-7.51^{\circ} \mathrm{C}$ \\
\hline
\end{tabular}

In the spherical Pore 1, the water in the $50 \mathrm{~nm}$ pore turned into ice at sub-cooling temperature in the range of $-2.34^{\circ} \mathrm{C} \leq T \leq-1.29^{\circ} \mathrm{C}$, while the rest of the pores within the system remained saturated. When sub-cooling temperature reduced to below -1.29 ${ }^{\circ} \mathrm{C}$, the ice bulged to a radius in the range of $30 \mathrm{~nm}$ to $50 \mathrm{~nm}$ without penetrated into the capillary pore channel 1 . Thus, the largest crystallization pressure in the spherical Pore 1 was generated with a magnitude of $1.15 \mathrm{MPa}$ at the Point A by using the Equation (1-4), which is equivalent to $P_{a 1}=\gamma_{C L}\left(2 /\left(r_{e 1}-\delta_{w}\right)-2 /\left(r_{s 1}-\delta_{w}\right)\right)$.

When the sub-cooling temperature reduced to $T=-2.34^{\circ} \mathrm{C}$, the ice was formed in the spherical Pore 2. The bulged ice free ends have a radius of $30 \mathrm{~nm}$ initially. When the sub-cooling temperature reached at $T=-3.58{ }^{\circ} \mathrm{C}$, the bulged ends were hemispherical with a radius of $20 \mathrm{~nm}$, as shown in Figure 5-3(b). The curvatures $\kappa^{e}$ at these two free hemispherical ends were equivalent to $2 /\left(r_{e 1}-\delta_{w}\right)$ to balance the interface energy. The largest crystallization pore pressure in the spherical Pore 1 was updated to $2.6 \mathrm{MPa}$ at 132 
Point A with the curvature change at the free end. The largest crystallization pore pressure in the spherical Pore 2 was generated with a magnitude of $1.48 \mathrm{MPa}$ at Point B by using the Equation (1-4), which is equivalent to $P_{a 2}=\gamma_{C L}\left(2 /\left(r_{e 2}-\delta_{w}\right)-2 /\left(r_{s 1}-\delta_{w}\right)\right)$.

As soon as the sub-cooling temperature reduced to below the threshold temperature or the freezing temperature at $T=-3.58^{\circ} \mathrm{C}$ for the capillary pore channel 1 , the bulged ice on both Pores 1 and 2 penetrated and merged in the capillary pore channel 1. When the temperature reduced to $T=-7.51^{\circ} \mathrm{C}$, the bulged ice in the cylindrical pore channel 1 has one hemispherical end with a radius of $10 \mathrm{~nm}$, as shown in Figure 5-3(c). The largest crystallization pore pressures in the spherical Pores 1 and 2 were calculated with magnitudes of $7.34 \mathrm{MPa}$ and $6.19 \mathrm{MPa}$ by using the Equation (1-4) which is equivalent to $P_{a 1}=\gamma_{C L}\left(2 /\left(r_{e 1}-\delta_{w}\right)-2 /\left(r_{s 2}-\delta_{w}\right)\right)$ and $P_{a 2}=\gamma_{C L}\left(2 /\left(r_{e 2}-\delta_{w}\right)-2 /\left(r_{s 2}-\delta_{w}\right)\right)$, respectively. The confining pressure was generated along the cylindrical wall of capillary pore channel 1 and was calculated as $6.86 \mathrm{MPa}$ with the Equation (1-4), which is equivalent to $P_{a 3}=\gamma_{C L}\left(2 /\left(r_{e 2}-\delta_{w}\right)-1 /\left(r_{e 1}-\delta_{w}\right)\right)$.

Finally, as the temperature further reduced to slightly below the threshold temperature $T=-7.51^{\circ} \mathrm{C}$, the bulged ice can penetrate into the capillary pore channel 2 . At this stage, the ice invaded through the cylindrical Pore 2 with the hemispherical free end. The largest crystallization pressures in spherical Pores 1 and 2, and the confining pressure in the cylinder pore channel 1 were the same as the previous case. The confining pressure along the cylindrical capillary pore channel 2 was calculated as $4.51 \mathrm{MPa}$ with the Equation (1-4), which is equivalent to $P_{a 4}=\gamma_{C L}\left(2 /\left(r_{e 2}-\delta_{w}\right)-1 /\left(r_{e 2}-\delta_{w}\right)\right)$, as shown in Figure 5-3(d). 
For the stage shown in Figure 5-3(d), all the applied pressures were sufficient to cause damages to the pore surfaces. In order to validate these theories, the XFEM fracture simulations were conducted in this pore system as shown in Figure 5-4 and Figure 5-5. Similar material properties and tensile strength that were defined in the Scenario 1 were applied. All models were defined with confined boundary conditions with roller supports along the edges of the models. In addition, an initial crack length of two elements was defined, which is equivalent to $2 \mathrm{~nm}$. Since the current XFEM program can only simulate one crack, the fracture simulations were carried out separately. For the spherical Pores 1 and 2, the fracture simulations were conducted at the weakest point, that is, Points A and B, respectively. The calculated crystallization pressure at Points A (7.34 MPa) and B (6.19 MPa) were input in the XFEM fracture simulation models. The fracture simulations were conducted based on the maximum principle stress criteria (Equations (5-1) and (5-2)) and the generated results are as shown in Figure 5-4 (a) and (b). For the cylindrical capillary pore channels 1 and 2 (Figure 5-5 (c) and (d)), the XFEM fracture simulations were conducted in the perpendicular surface with applied crystallization pore pressure along the cylindrical wall at Points C (6.86 MPa) and D (4.51 MPa), respectively. These points were assumed to be the weakest points or the defective points. The simulation results had shown that all cracks extended which indicated the computed pressures were sufficient to overcome the fracture criteria and cause damages on cement paste. 
(a)

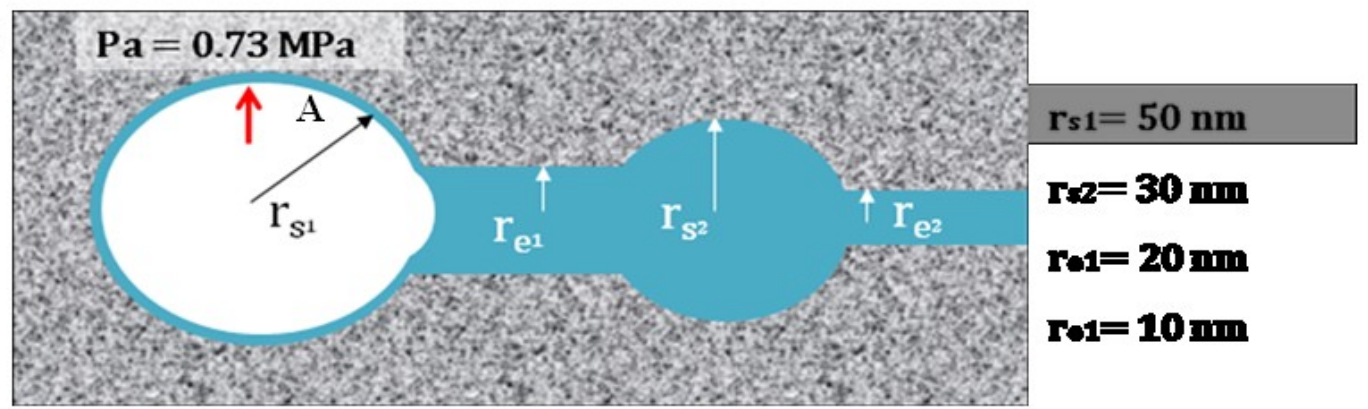

(b)

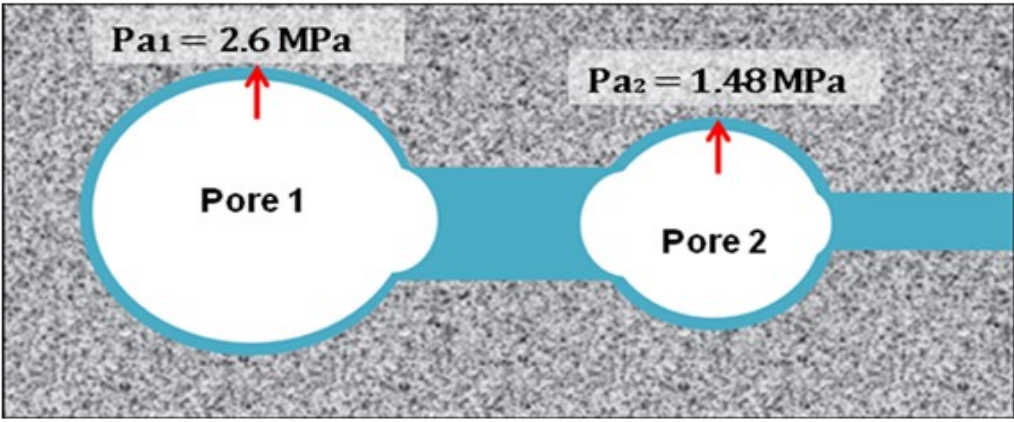

(c)

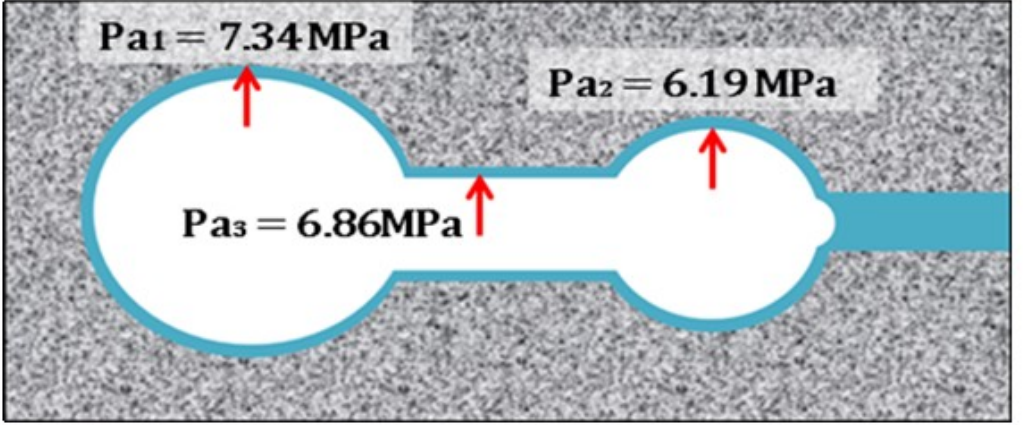

(d)
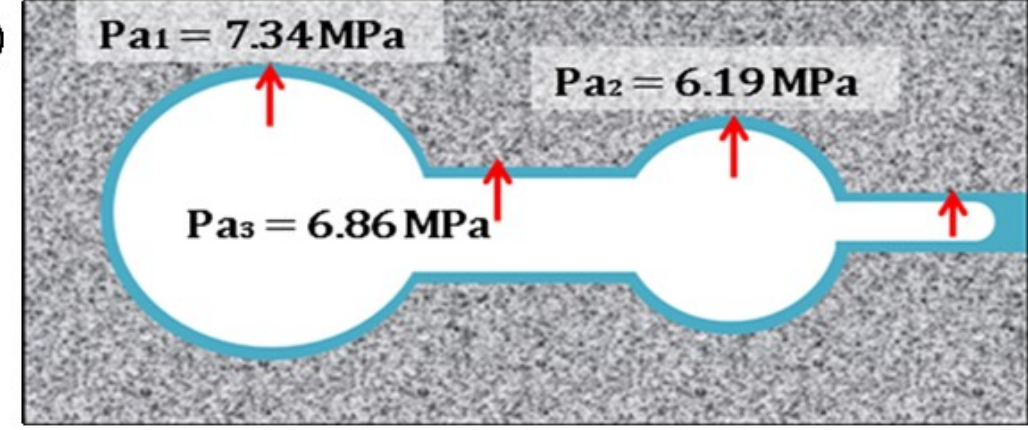

Pas $=4.51$ MPa

Figure 5-3: Schematic illustration of multiple pore system with capillary pores. (a) At $T=-2{ }^{\circ} \mathrm{C}$, ice was formed in spherical pore 1 and bulged into the cylindrical pore channel 1 , (b) at $T=-3.58{ }^{\circ} \mathrm{C}$, ice was formed in Pore 2 and bulged into the cylindrical pore channel 1 with hemispherical free ends, (c) at $T=-7.51{ }^{\circ} \mathrm{C}$, ice invaded through cylindrical pore channel 1 and bulged into cylindrical pore channel 2 with hemi-sphere ends, and (d) slightly below threshold temperature $T=-7.51^{\circ} \mathrm{C}$, the ice is penetrating into the cylindrical pore channel 2 with one free hemispherical end. 
(a)

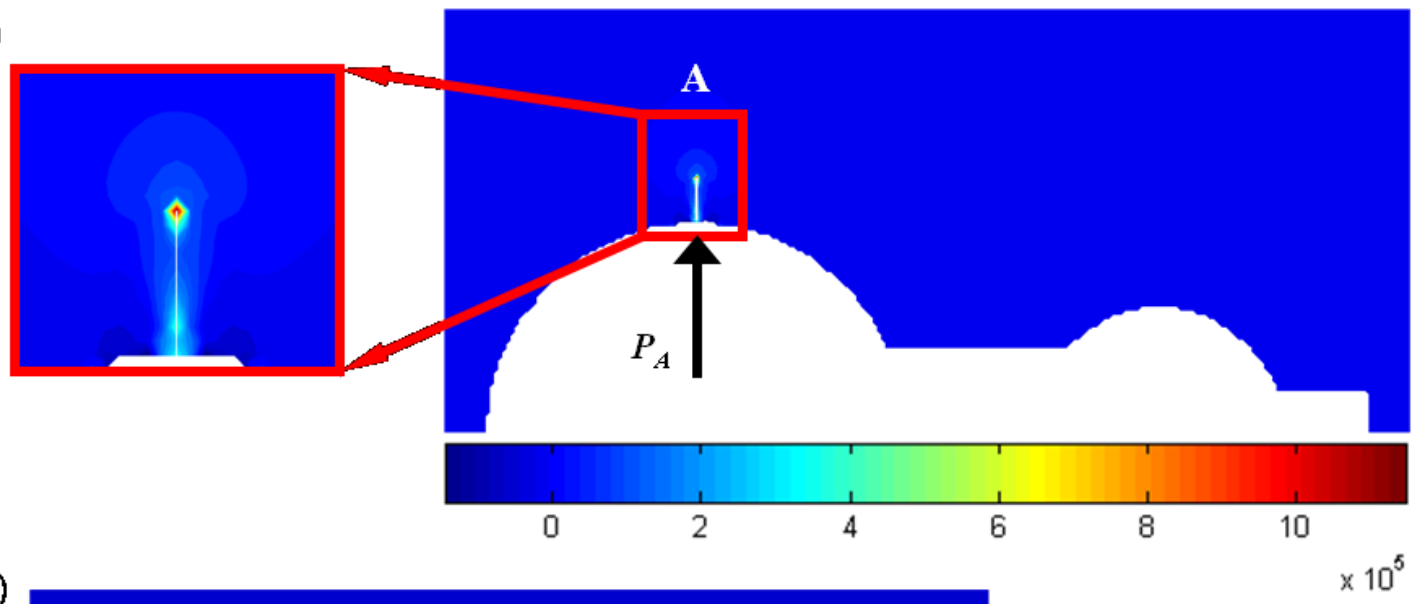

(b)

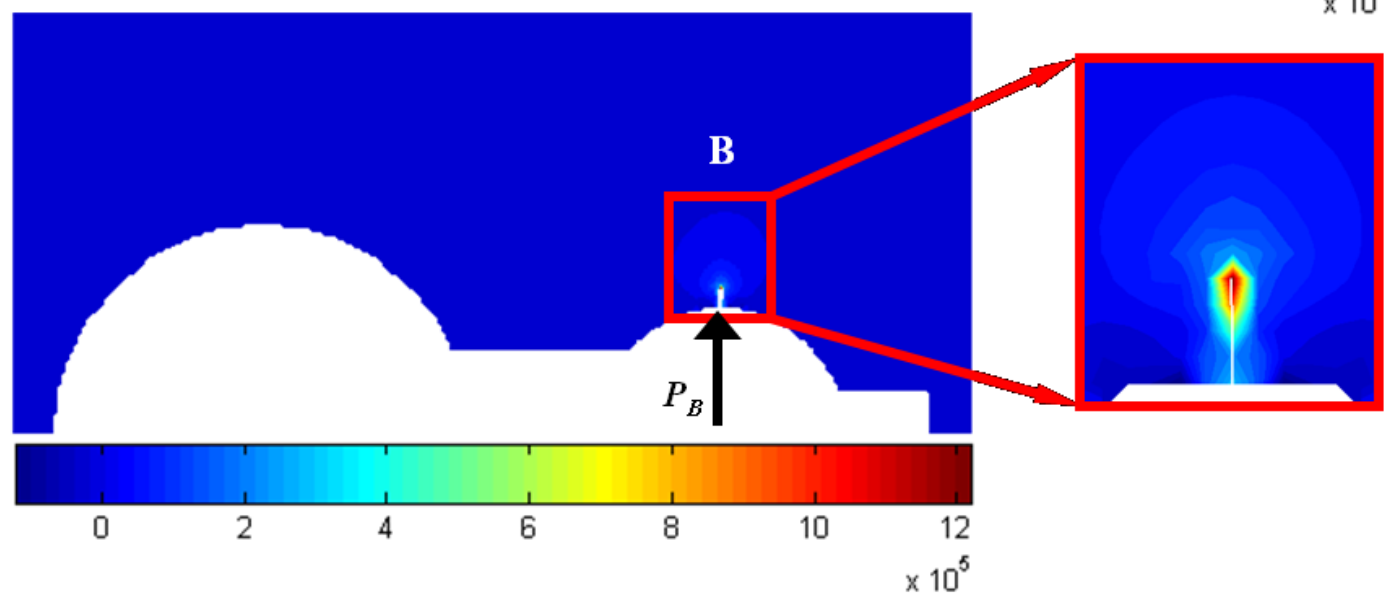

Figure 5-4: XFEM simulation $\sigma_{1}$ contour plot for multiple pores scenario with crack generated since the hoop stress generated was greater than the tensile strength of cement paste. Simulation results at temperature slightly below $-7.51{ }^{\circ} \mathrm{C}$ in: (a) Pore 1 with $P_{A}=7.34 \mathrm{MPa}$ at point $\mathrm{A}$ and (b) Pore 2 with $P_{B}=6.19 \mathrm{MPa}$ at Point B. 
(a)

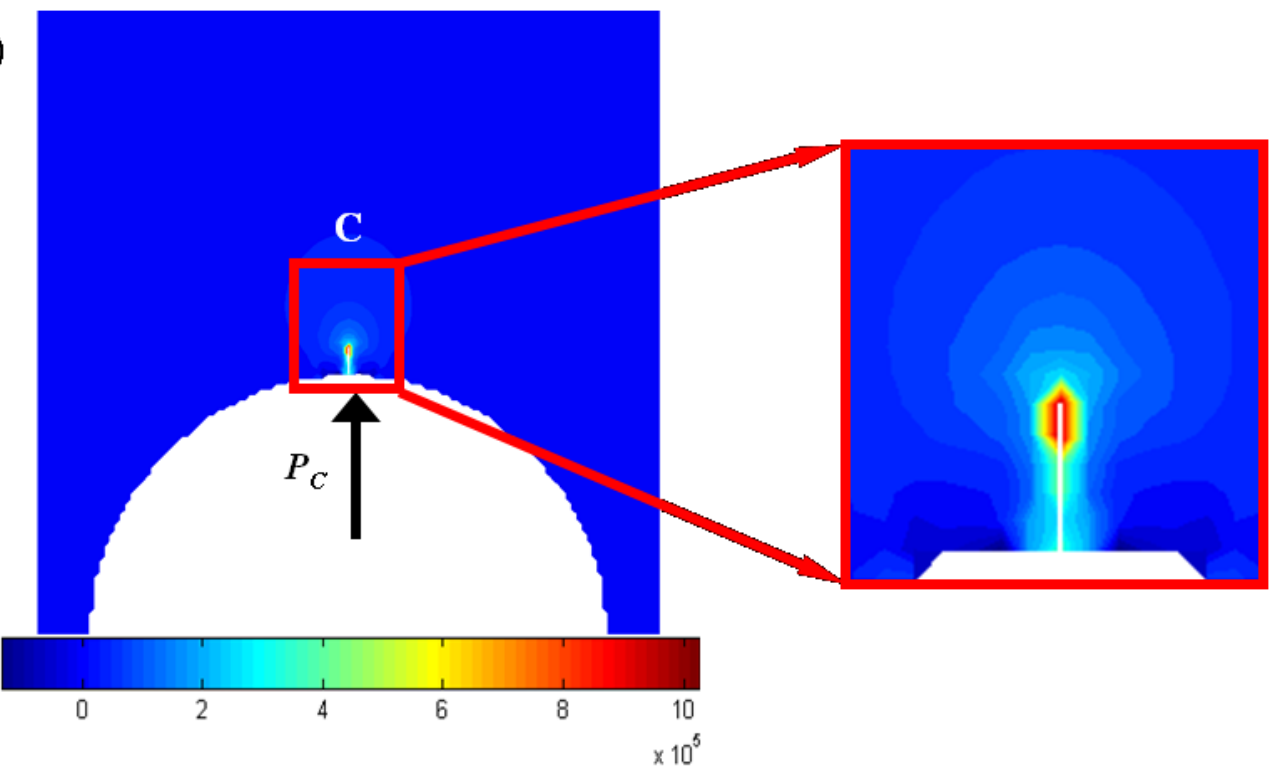

(b)

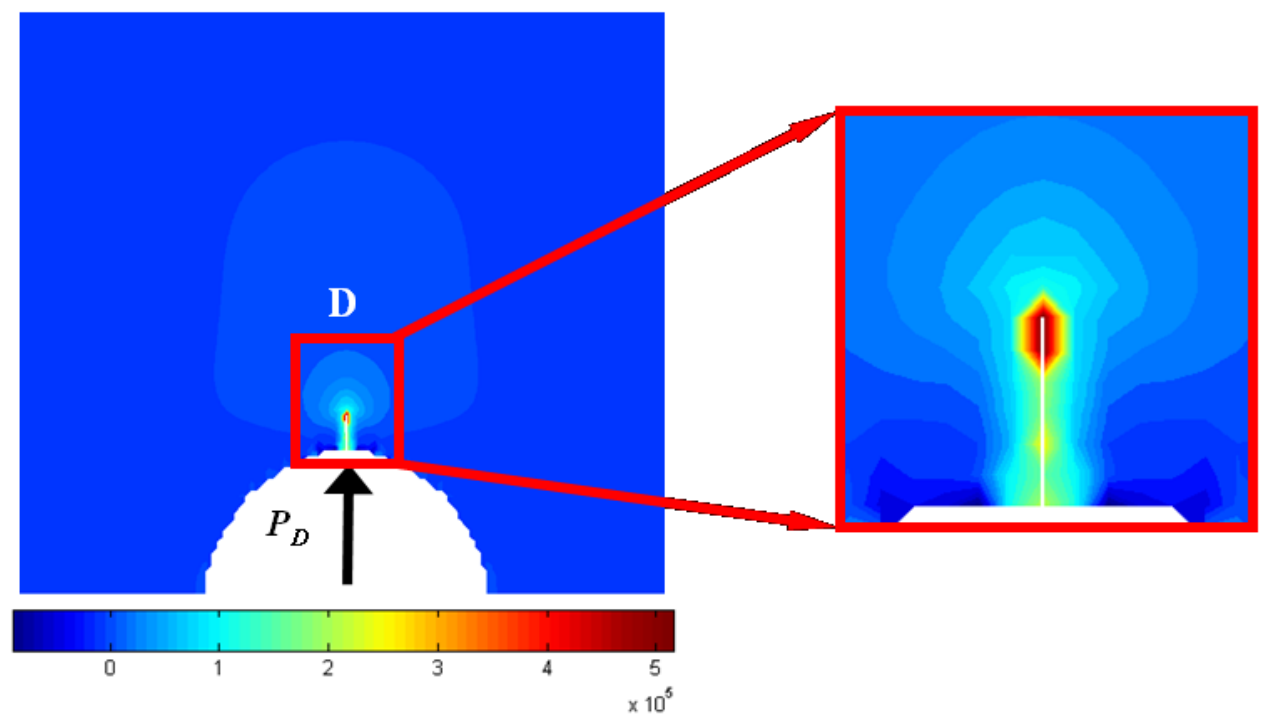

Figure 5-5: XFEM fracture simulations $\sigma_{1}$ contour plot on cylindrical capillary pore channels 1 and 2 at the temperature slightly below $-7.51^{\circ} \mathrm{C}$ in: (c) Channel 1 with $P_{C}=$ $6.86 \mathrm{MPa}$ at point $\mathrm{C}$, and (d) Channel 2 with $P_{D}=4.51 \mathrm{MPa}$ at point $\mathrm{D}$. The views for both channels 1 and 2 are in the plane perpendicular to this page. 


\subsection{3-D Fracture Simulation of Internal Frost Damage within Idealized Pore System}

The limitation of XFEM technique prevented the fracture simulation to be performed to model the cracks to coalesce in a porous media. Alternatively, the bilinear CZM technique can be employed. Prior performing 3-D fracture simulation, a 2-D case was performed to check the capability of the bilinear CZM technique to simulate internal frost damage on porous cement paste.

\subsubsection{2-D damage simulation with one idealized pore in cement paste sample}

The rectangular sample with dimensions $50 \mu \mathrm{m} \times 50 \mu \mathrm{m}$ were generated for 2-D CZM modeling as shown in Figure 5-7. A spherical pore with $10 \mu \mathrm{m}$ in radius was constructed. All the edges were defined with roller supports to create a confined boundary. Four-node bilinear square element having a size of $1 \mu \mathrm{m} \times 1 \mu \mathrm{m}$ was used in the model. Figure 5-6 shows the surface numbering scheme on an element with respect to the node locations in the ABAQUS.

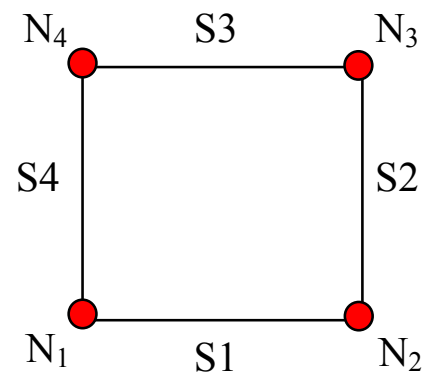

Figure 5-6: Schematic illustration of node $\left(\mathrm{N}_{1}, \mathrm{~N}_{2}, \mathrm{~N}_{3}\right.$, and $\left.\mathrm{N}_{4}\right)$ and surface (S1, $\mathrm{S} 2, \mathrm{~S} 3$, and $\mathrm{S} 4$ ) numbering of a four-node bilinear square element. 
The material properties for the cement paste were defined with Young's Modulus and Poisson's ratio of $22 \mathrm{GPa}$ and 0.25 , respectively. A predefined crack path with bilinear CZM elements were defined on top of the pore at location A, as shown in Figure 5-7. The fracture energy and tensile strength for cement paste material were defined as $37.5 \mathrm{~J} / \mathrm{m}^{2}$ and $0.15 \mathrm{GPa}$, respectively for the bilinear CZM parameters. Assuming the pore was connected to a capillary pore with $5 \mathrm{~nm}$ in radius, the crystallization pressure was determined to be approximately $20 \mathrm{MPa}$ based on the Equation (1-4), when the ice bulges into the capillary pore at the sub-cooling temperature of $-16.7{ }^{\circ} \mathrm{C}$ (Equation (1-1)). The thermodynamic principle is used to computing the crystallization pore pressure, which is correlated to the pore radius and temperature. A distributed pressure surface load (*DSLOAD) was applied on the pore surfaces based on the ABAQUS scheme. Figure 5-7 shows the result of 2-D fracture simulation generated with crack opening in the 10 $\mu \mathrm{m}$ pore in ABAQUS. The result proved that the bilinear CZM technique can be employed to study the internal frost damage in cement paste. 


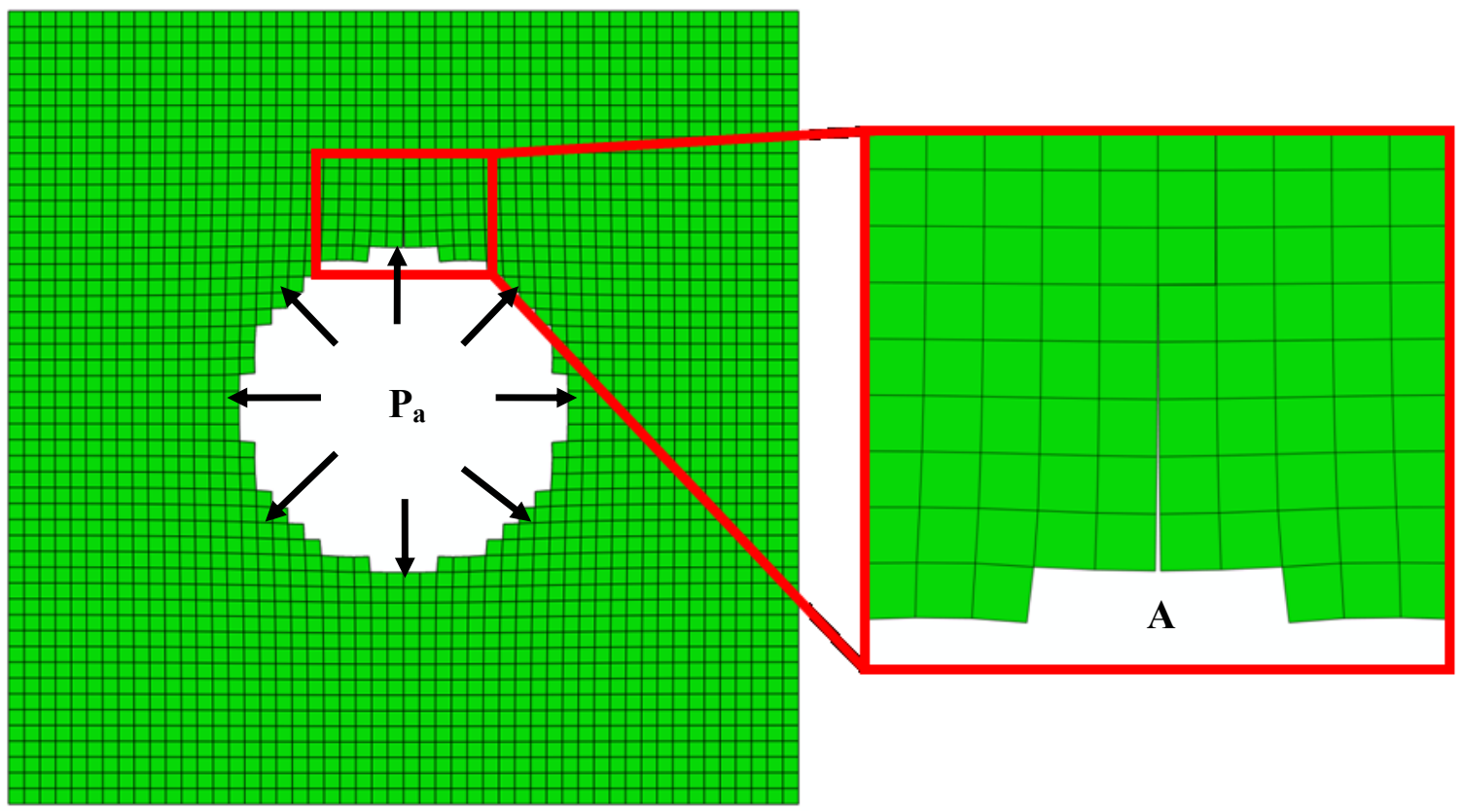

Figure 5-7: A 2-D bilinear CZM fracture simulation in ABAQUS on an idealized pore with $10 \mu \mathrm{m}$ in radius in cement paste as a result of crystallization pore pressure, $\mathrm{P}_{\mathrm{a}}$. The enlarged view showed the crack opening at Point A as a result of crystallization pore pressure.

\subsubsection{3-D damage simulation with one idealized pore in cement paste sample}

In developing 3-D fracture model, three image slices were utilized. Similar geometry, boundary conditions, material properties, and bilinear CZM fracture parameters from the 2-D case were applied in the 3-D model. The thickness of this sample is $3 \mu \mathrm{m}$. The cubic 8 -node reduced integration element (C3D8R) was employed for mesh construction. Figure 5-8 shows the surface numbering with respect to the node locations in a 3-D element. Similar to the 2-D case, a crystallization pore pressure of 2 $\mathrm{MPa}$ was evaluated on the Pore A surfaces at the sub-cooling temperature of $16.7{ }^{\circ} \mathrm{C}$, when the ice bulges into the capillary pore. Figure 5-9 shows the simulation result generated in ABAQUS with the pore in the cement paste experiencing crack opening due 
to induced crystallization pore pressure effect. Hence, the simulation result indicated that the bilinear CZM technique can be employed to perform internal frost damage analysis on cement paste in a 3-D model. In Chapter 2, it had been observed that the bilinear CZM technique was capable to simulate fracture simulation in a porous media. Thus, it is feasible to perform frost induced damage analysis in a porous cement paste by using the bilinear CZM technique in 2-D and 3-D models.

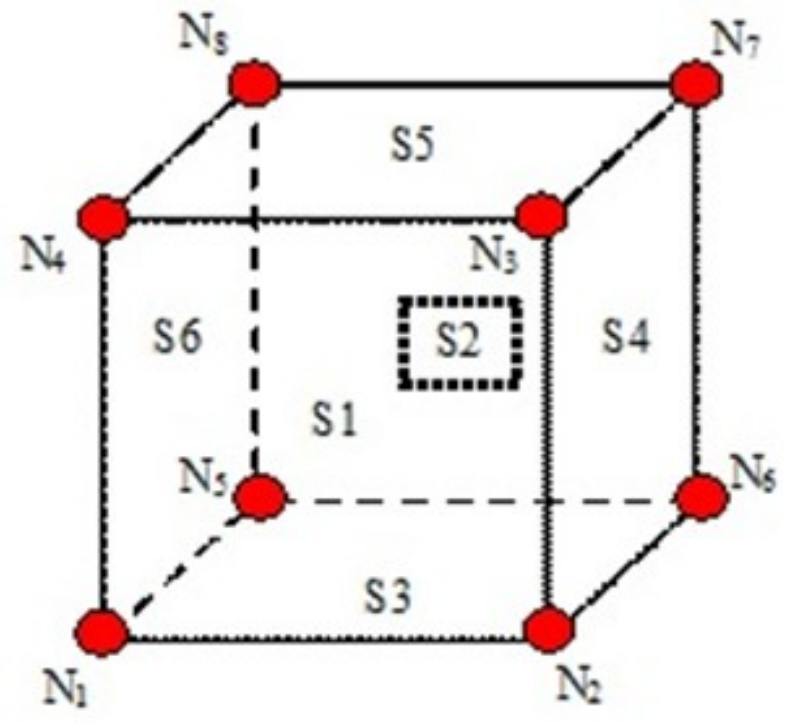

Figure 5-8: Schematic illustration of node and surface numbering of a cubic 8-node reduced integration element. 


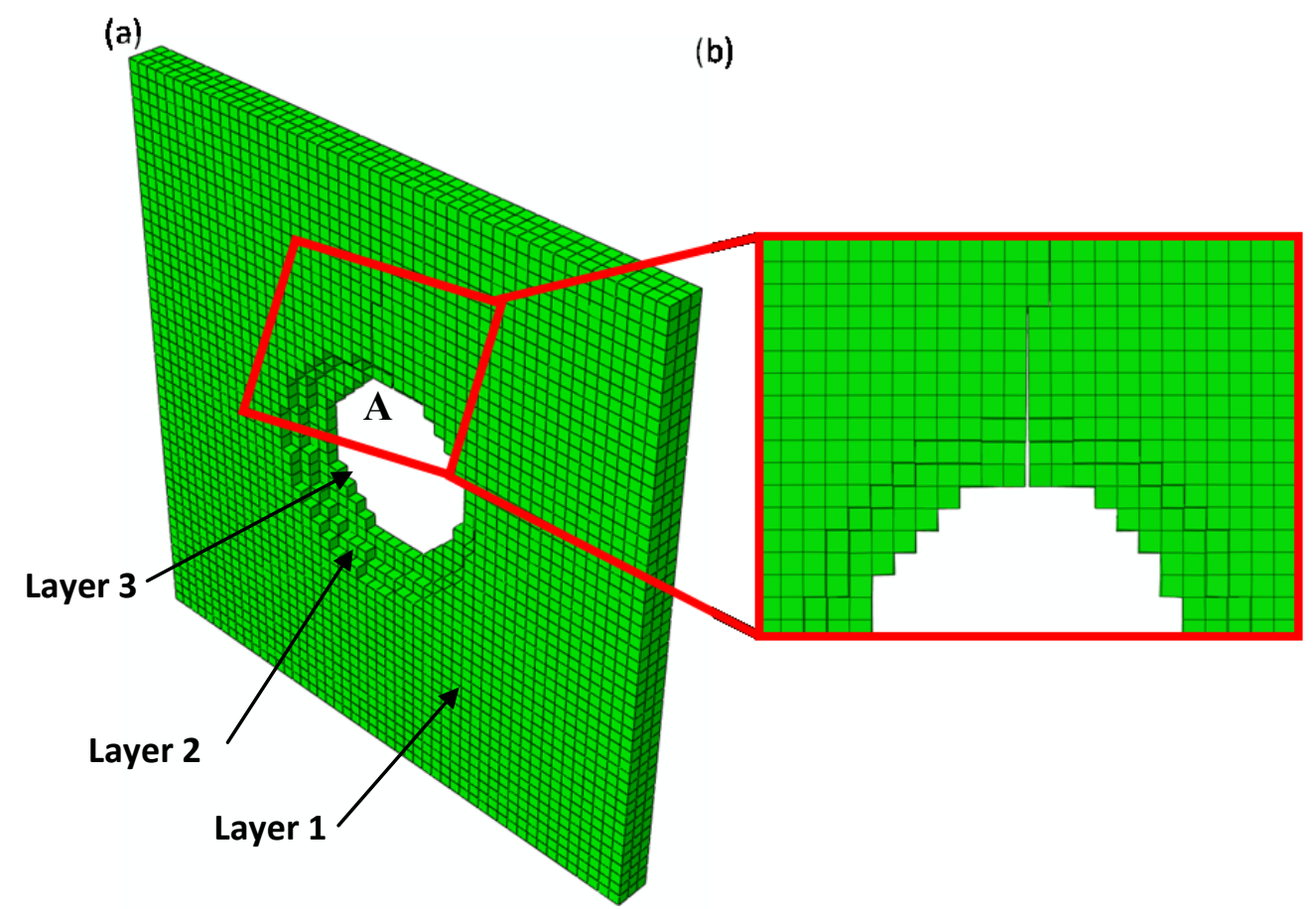

Figure 5-9: A 3-D bilinear CZM fracture simulation in ABAQUS on an idealized pore with $10 \mu \mathrm{m}$ in radius in cement paste as a result of crystallization pressure.

\subsection{Concluding Remarks}

Internal frost damage is a major factor that affects the concrete durability in cold regions. This chapter investigates crystallization pore pressure and the sequential internal frost damage of idealized cementitious material samples with thermodynamic analysis. It is believed that the crystallization pressure due to the ice nucleation inside of the capillary pores can cause the crack initiation and propagation. Under sub-cooling temperatures, the crystallization pressures inside idealized pore system were calculated with thermodynamic theories, which correlate to the pore curvature.

The damage processes due to ice crystallization were demonstrated with two idealized pore systems under reduced sub-cooling temperature. The ice geometry 
development and the generated crystallization pore pressure were used for XFEM fracture simulation with maximum normal stress criterion. The analytical study on these idealized cement samples demonstrated the ice formation and internal frost damage process.

Both XFEM and bilinear CZM techniques were applied with the thermodynamic principles for the internal frost damage analysis and crystallization pore pressure prediction. In the XFEM fracture models, the simulations results in both scenarios (Single pore and multi-pores systems) had proven that the computed crystallization pore pressures were sufficient to overcome the fracture criteria of the cement paste. The bilinear CZM technique was employed to perform damage simulation in the porous cement paste to overcome the XFEM technique. The crack openings on both 2-D and 3D models in ABAQUS had shown that with the calculated crystallization pore pressure, the bilinear CZM model can simulate internal frost damage in the porous cement paste. 


\section{CHAPTER 6 VALIDATION OF MICROMECHANICAL ANALYSIS OF INTERNAL FROST DAMAGE AND FRACTURE TESTS WITH TESTED SAMPLES}

\subsection{Overview}

All the developed computational tools incorporating the theoretical analysis were used to simulate fracture modeling on concrete and cement paste specimens. The techniques that were utilized for simulating the fracture analysis were XFEM and bilinear CZM. This is because of these techniques are capable to simulate fracture behavior in both idealized homogeneous and heterogeneous material samples, as presented in Chapter 2. Similarly, the internal frost damage analysis using the thermodynamic principles in Chapter 5 had proven that the computed crystallization pore pressure can lead to damage on cement paste specimens. The crack growth trend was employed as the technique to validate the computational tool results with the actual damage behavior on the concrete and cement paste specimens. The XFEM fracture simulation was conducted on the acquired digital sample after performing imaging process and elliptical fitting technique for generating idealized inclusions. On the other hand, the bilinear CZM fracture models were developed through acquired digital sample from image processing with the shape of the inclusions were remained the same as the actual sample. The XFEM simulation results were verified for the fracture behavior in both middle-notched SEB and freeze-thaw test on cement paste samples. The bilinear CZM also successfully simulated SEB test in 2-D and internal frost damage analysis in 2-D and 3-D models. All the simulation results were 
validated by comparing model prediction with the actual tested samples through the crack growth paths.

\subsection{Computational Prediction and Validation with Concrete SEB Test}

The image of undamaged concrete SEB test sample was utilized to develop FE model through image processing as presented in Chapter 4 Section 4.2. The elliptical fitting technique was employed to generate idealized elliptical inclusions in the FE model. The developed XFEM model for the SEB bending test was executed to generate crack propagations in the concrete specimen. Figure 6-1 (Ng and Dai 2012) demonstrates the XFEM fracture simulation result on concrete SEB bending test. Figure 6-1(b) shows the crack growth trend developed in the simulated XFEM mesh model that employed the XFEM technique, which incorporated the search distance and kink angle techniques (Dai and Ng 2010; Ng and Dai 2012). The highest energy released rate was employed to guide the crack growth direction, with a total of 55 iterations were executed for the simulation. As shown in Figure 6-1, both simulated result and the actual tested SEB test (as indicated with red line in Figure 6-1(a)) display a well agreement in crack growth trend. Thus, it can be concluded that the XFEM fracture simulation result was validated and the technique is capable to perform fracture simulations not only in homogeneous but also heterogeneous samples. 

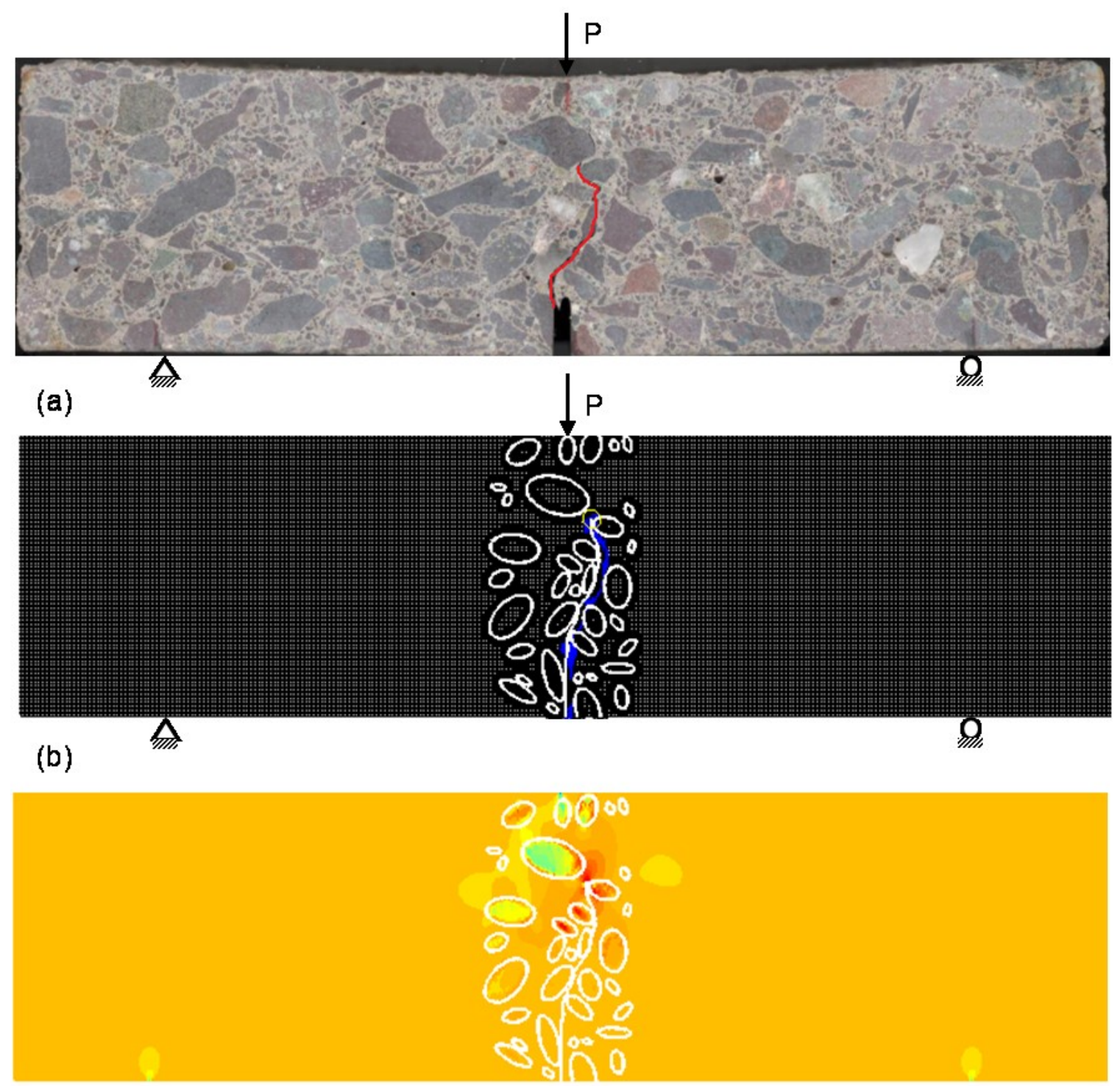

(c)

Figure 6-1: XFEM simulation of singe-edge middle-notched beam test on a concrete specimen: (a) image of the tested specimen with indicated propagated crack line shown in red, (b) mesh plot with predicted crack path in the digital sample, (c) $\sigma_{y y}$ stress contour with crack propagation (Ng and Dai 2012) - see permission in Appendix C3.

On the other hand, the bilinear CZM technique was also employed to simulate the fracture behavior of the concrete SEB bending test. Rather than defining idealized inclusions, the bilinear CZM FE model maintained the original inclusion complex shapes 
of the actual tested sample through image processing, as discussed in Chapter 4 Section 4.2. The material properties for the concrete beam are listed in Table 6-1 (Mehta and Monteiro 2006). For cement matrix, the Young's modulus and Poisson's ratio are $20 \mathrm{GPa}$ and 0.35 , respectively. For aggregate, the Young's modulus and Poisson's ratio are 50 GPa and 0.35 , respectively. For homogeneous properties of concrete, the Young's modulus and Poisson's ratio are $35 \mathrm{GPa}$ and 0.35 , respectively. The parameters for bilinear CZM elements were also defined as listed in Table 6-1 (Roesler et al. 2007). For the cement matrix bonding, the fracture energy and tensile strength were $37.5 \mathrm{~J} / \mathrm{m}^{2}$ and $3 \mathrm{GPa}$, respectively. For the aggregate bonding, the fracture energy and tensile strength were $14.0 \mathrm{~J} / \mathrm{m}^{2}$ and $10 \mathrm{GPa}$, respectively. Whereas, for the cement-aggregate interface, the fracture energy and tensile strength were $25 \mathrm{~J} / \mathrm{m}^{2}$ and $1 \mathrm{GPa}$, respectively. The critical strain ratio of $\lambda_{c r}=0.25$ was employed.

Table 6-1: Material properties and cohesive fracture parameters for different phases in concrete (Mehta and Monteiro 2006; Roesler et al. 2007)

\begin{tabular}{|c|c|c|c|c|}
\hline Phases & $\begin{array}{c}\text { Young's } \\
\text { Modulus (GPa) }\end{array}$ & $\begin{array}{c}\text { Poisson's } \\
\text { Ratio }\end{array}$ & $\begin{array}{c}\text { Fracture Energy } \\
\left(\mathbf{J} / \mathbf{m}^{\mathbf{2}}\right)\end{array}$ & $\begin{array}{c}\text { Tensile } \\
\text { Strength } \\
\mathbf{( G P a )}\end{array}$ \\
\hline Cement Matrix & 20 & 0.35 & 37.5 & 3 \\
\hline Aggregate & 50 & 0.35 & 14.0 & 10 \\
\hline Concrete & 35 & 0.35 & N/A & N/A \\
\hline $\begin{array}{c}\text { Cement-Aggregate } \\
\text { Interface }\end{array}$ & N/A & N/A & 25 & 1 \\
\hline
\end{tabular}

Figure 6-2 illustrates the fracture simulations generated in the ABAQUS by using the bilinear CZM technique. Figure 6-2(a) shows the deformation and fracture pattern 
developed after a unit displacement was applied to the model. Figure 6-2(b) shows the enlarged view of the crack growth trend developed in the model. The result shows that the crack developed mostly along the interface of cement-aggregate, which holds true as the bonding strength is the weakest on the interface of aggregate-cement. Overall, the result also shows a well agreement to the actual concrete SEB bending test result (Figure 6-1(a)). Thus, it was validated that the bilinear CZM technique also can be employed to simulate any fracture test model on concrete samples with proper boundary conditions defined. 
(a)

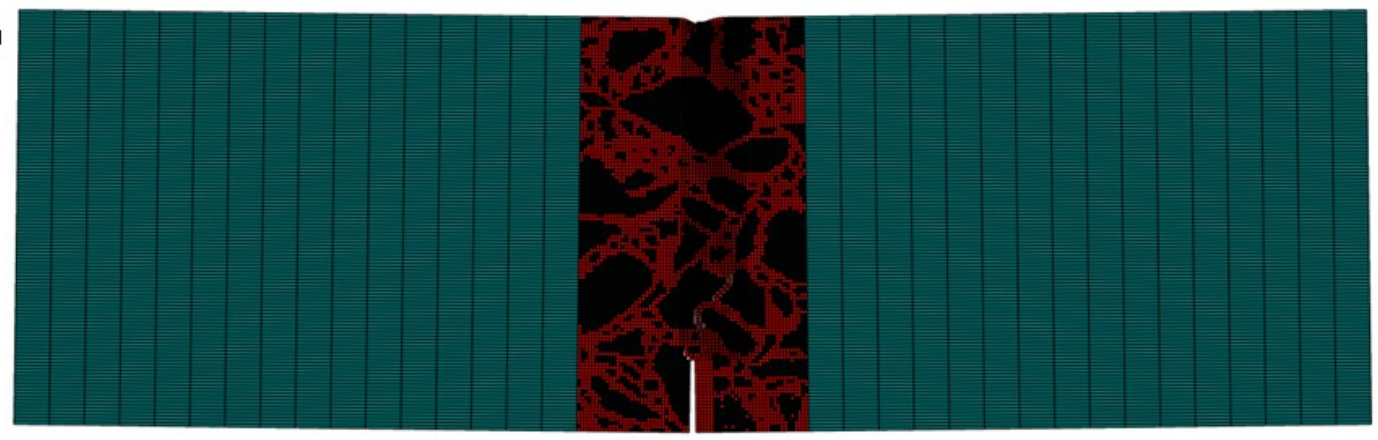

(b)

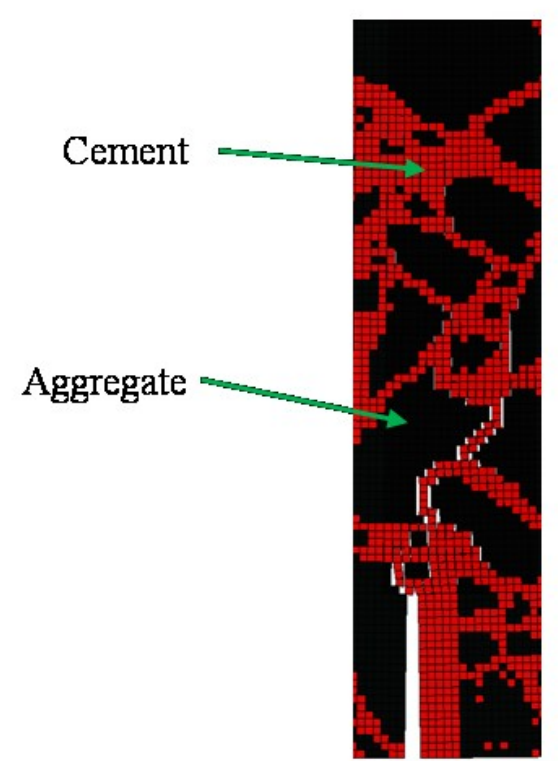

Figure 6-2: Fracture simulation of the concrete SEB test by using bilinear CZM technique in ABAQUS. (a) The SEB model in ABAQUS with deformation and fracture developed after a unit displacement is applied on the model. (b) A section of detail view of the SEB model that displays the crack growth pattern developed mostly along the cement-aggregate interface.

\subsection{Computational prediction of fracture test and internal frost damage with 2-D SEM image samples}

The fracture test and internal frost damage computational analysis were carried out on SEM images of cement paste sample by using bilinear CZM technique, which was executed in ABAQUS. The CT test simulation was firstly performed for the fracture test 
analysis on an undamaged cement paste sample to prove the capability of bilinear CZM technique on modeling fracture behavior in a porous media. Next, the internal frost damage analysis was performed on a damaged cement paste sample to validate the fracture behavior induced by crystallization pore pressure through the crack growth path development.

\subsubsection{2-D SEM digital sample generation}

The 2-D SEM digital sample generation was firstly carried out through image processing, such as filtering technique for noise reduction in the image. The processed image was then read in the developed MATLAB scheme to construct mesh based on the intensity (0-255) of the image. An ABAQUS input file was then generated after defining the boundary conditions and material properties in the MATLAB program. Chapter 4 Section 4.3 presented a complete SEM digital sample generation. The material properties for the concrete beam are listed in Table 6-2. For hydrated cement, the Young's modulus and Poisson's ratio are $22 \mathrm{GPa}$ and 0.25 , respectively. For unhydrated cement, the Young's modulus and Poisson's ratio are $135 \mathrm{GPa}$ and 0.35 , respectively. The parameters

for bilinear CZM elements were also defined as listed in Table 6-2 (Liu et al. 2011). For hydrated cement bonding, the fracture energy and tensile strength were $37.5 \mathrm{~J} / \mathrm{m}^{2}$ and $0.15 \mathrm{GPa}$, respectively. For aggregate bonding, the fracture energy and tensile strength were $14.0 \mathrm{~J} / \mathrm{m}^{2}$ and $1.80 \mathrm{GPa}$, respectively. Whereas, for cement-aggregate interface, the fracture energy and tensile strength were $25 \mathrm{~J} / \mathrm{m}^{2}$ and $0.05 \mathrm{GPa}$, respectively. The critical strain ratio of $\lambda_{c r}=0.25$ was employed. 
Table 6-2: Material properties and cohesive fracture parameters for different phases in cement paste (Liu et al. 2011).

\begin{tabular}{|c|c|c|c|c|}
\hline Phases & $\begin{array}{c}\text { Young's } \\
\text { Modulus } \\
\text { (GPa) }\end{array}$ & $\begin{array}{c}\text { Poisson's } \\
\text { Ratio }\end{array}$ & $\begin{array}{c}\text { Fracture } \\
\text { Energy } \\
\left(\mathbf{J} / \mathbf{m}^{2}\right)\end{array}$ & $\begin{array}{c}\text { Tensile } \\
\text { Strength } \\
(\mathbf{G P a})\end{array}$ \\
\hline Hydrated Cement & 22 & 0.25 & 37.5 & 0.15 \\
\hline Unhydrated Cement & 135 & 0.35 & 14.0 & 1.80 \\
\hline $\begin{array}{c}\text { Hydrated-Unhydrated } \\
\text { Cement Interface }\end{array}$ & N/A & N/A & 25 & 0.05 \\
\hline
\end{tabular}

\subsubsection{Computational simulation of 2-D SEM digital samples under CT test}

In order to determine the capability of bilinear CZM technique to simulate crack coalesce in a porous media, a CT test model was utilized here to perform the fracture modeling. As presented in Chapter 4 section 4.3, a section of SEM image (Figure 4-3) of an undamaged cement paste sample with $\mathrm{w} / \mathrm{c}=0.47$ was used for developing the $\mathrm{CT}$ test model. The image section was used as some pores were aligned and has higher possibility to occur coalescing when internal frost damage occurred. Figure 4-4 in section 4.3 shows the CT model generated from the undamaged cement paste SEM image (Figure 4-3) based on the standard dimension. Figure 6-3 shows the von Mises stress contour plot of the $\mathrm{CT}$ test fracture simulation result at the initial and final stages of the process after a unit displacement was applied on the left hole and a fixed support on the right hole. The result implied that $\mathrm{CZM}$ technique can simulate the crack growth path not only on a heterogeneous material but also in a porous media. 
(a)
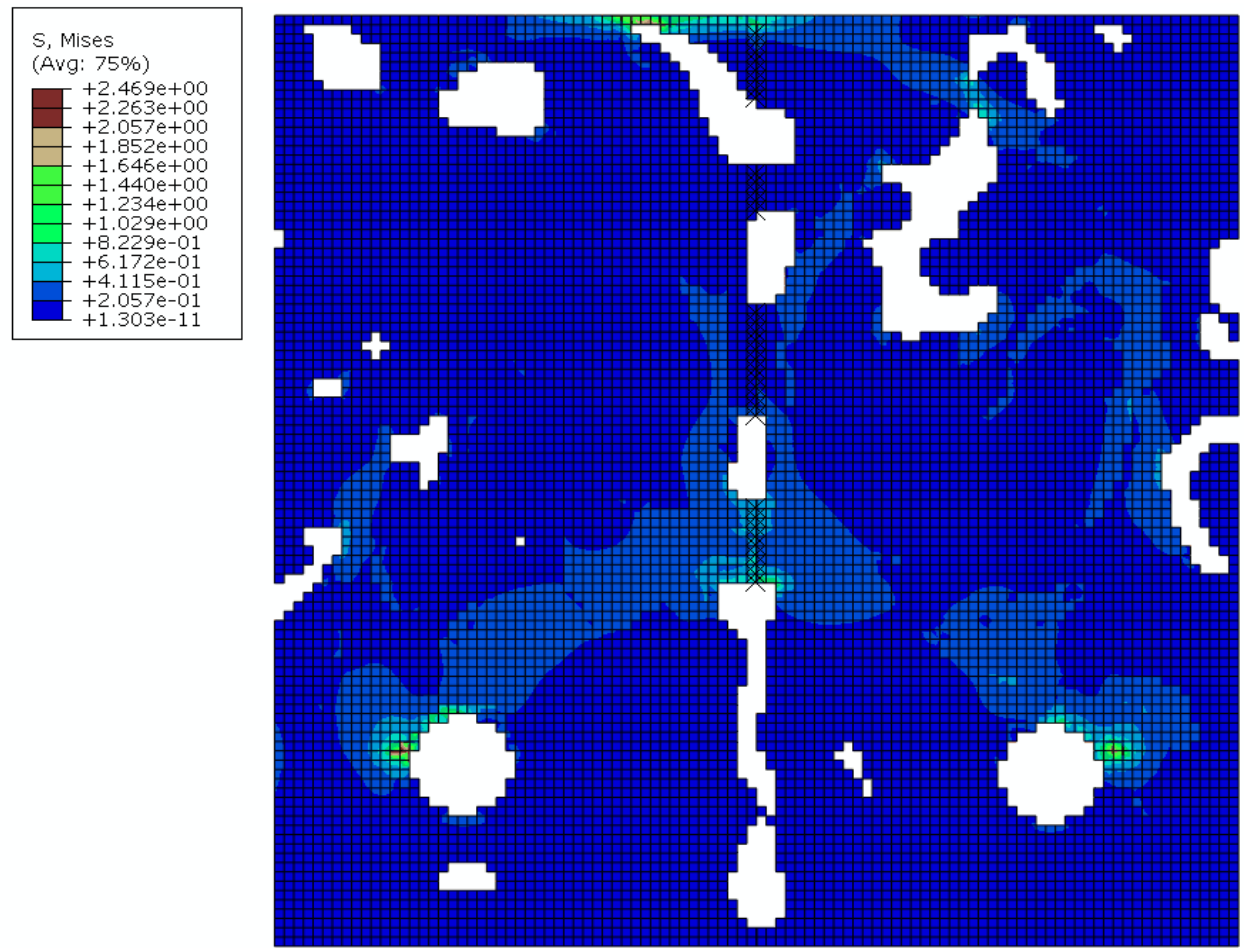

(b)
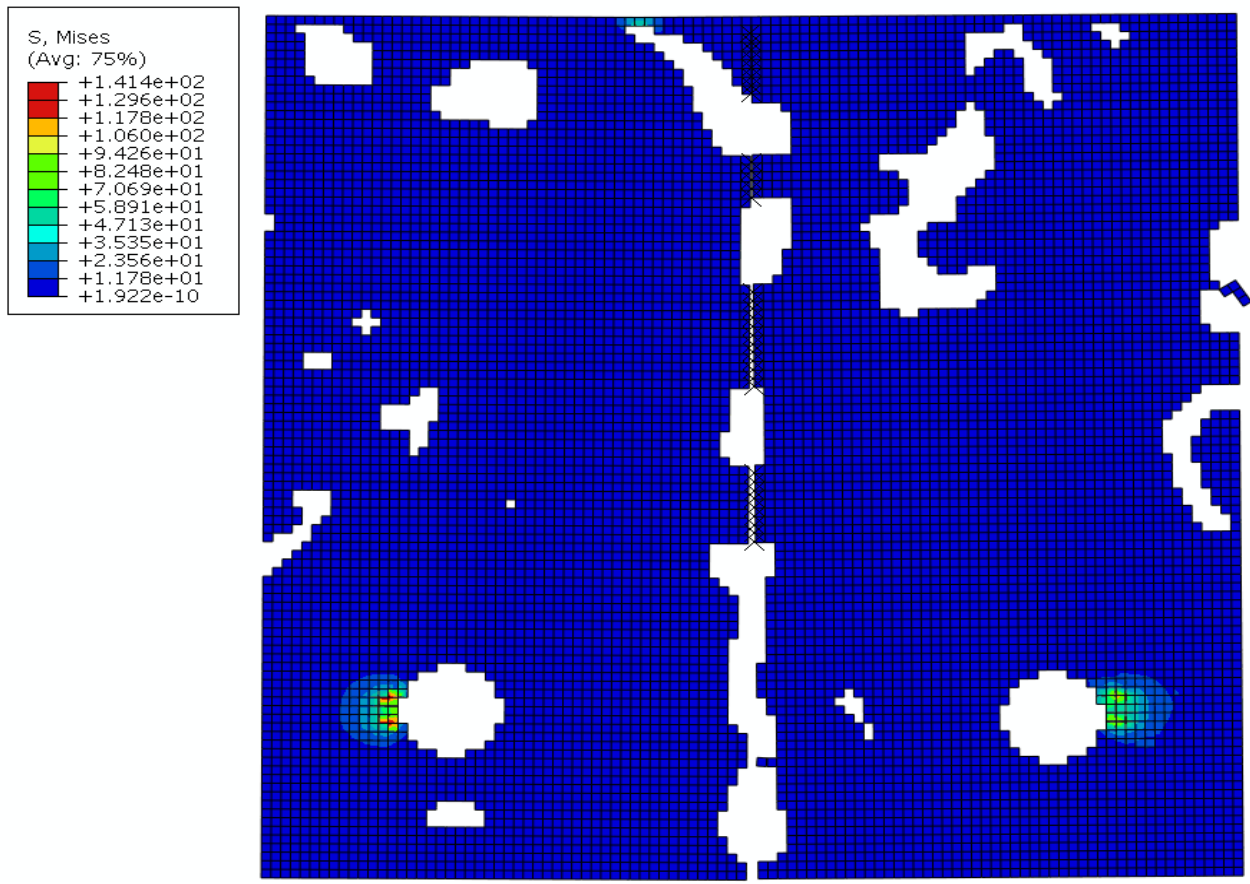

Figure 6-3: CZM fracture simulation of CT test with SEM digital cement paste sample. (a) The von Mises stress contour plot of the FE model at the initial stage of CT test. (b) At the final stage of the FE model, the CT test experiences complete failure with concentrated stress around the loading and fixed points. 


\subsubsection{Validation of internal frost damage 2-D simulation of SEM digital freeze- thawed damaged samples}

The successful fracture modeling of the porous media in the previous case had driven a good direction for performing internal frost damage simulation on cement paste sample. As presented in Chapter 4 Section 4.3, an image of a damaged cement paste sample with w/c $=0.47$ was successfully acquired using SEM, as shown in Figure 4-5(a). The developed cracks observed in the image were expected to be generated by the crystallization pore pressure effect in the sub-cooling temperature. This is because the developed crack was surrounded with pores. Thus, a section of interest was selected for performing the validation of internal frost damage analysis on the damaged cement paste sample, as shown in Figure 4-5(b). The actual crack path was patched except the Pore A at the bottom of the image, as shown in Figure 4-5(c). The Pore A was assumed to be the crack initiation location in the damaged cement paste sample.

After image processing was performed on the sectioned SEM image for noise reduction, a FE model was constructed with multiple columns of bilinear CZM elements defined on top of the Pore A, as highlighted in the yellow dotted box in Figure 6-4(a). An average pore radius was defined as $500 \mathrm{~nm}$ for Pore A. The radius was approximated by counting the number of elements away from the center of the pore curvature. Similar bilinear CZM properties from the CT test model were defined based on the material interface, as listed in Table 6-2. In boundary conditions, roller supports were defined around the edges of the model. By assuming a connecting pore of $10 \mathrm{~nm}$ in radius and using the Equation (1-4), the crystallization pore pressure at Pore A, $P_{A}$ was computed to be equivalent to $8.84 \mathrm{MPa}$. The sub-cooling temperature was estimated to be approximately $-7.51^{\circ} \mathrm{C}$ (Equation (1-1)). 
As shown in Figure 6-4 (b), the induced crystallization pore pressure on Pore A was sufficient to cause damage and generate cracks. In order to predict the crack growth direction in the multiple columns of CZM elements, the magnitudes of crack opening on the hydrated-unhydrated cement interface along the same row were compared. By comparing Figure 6-4 (c) to Figure 6-4 (d), it is clearly shown that the crack opening of Figure 6-4(d) in Row 1 was wider. Whereas, the crack opening of Figure 6-4 (c) in Row 2 was wider compared to the crack opening in Figure 6-4(d) Thus, based on this approach, the crack was predicted to grow to the left initially and then switched to the right. 


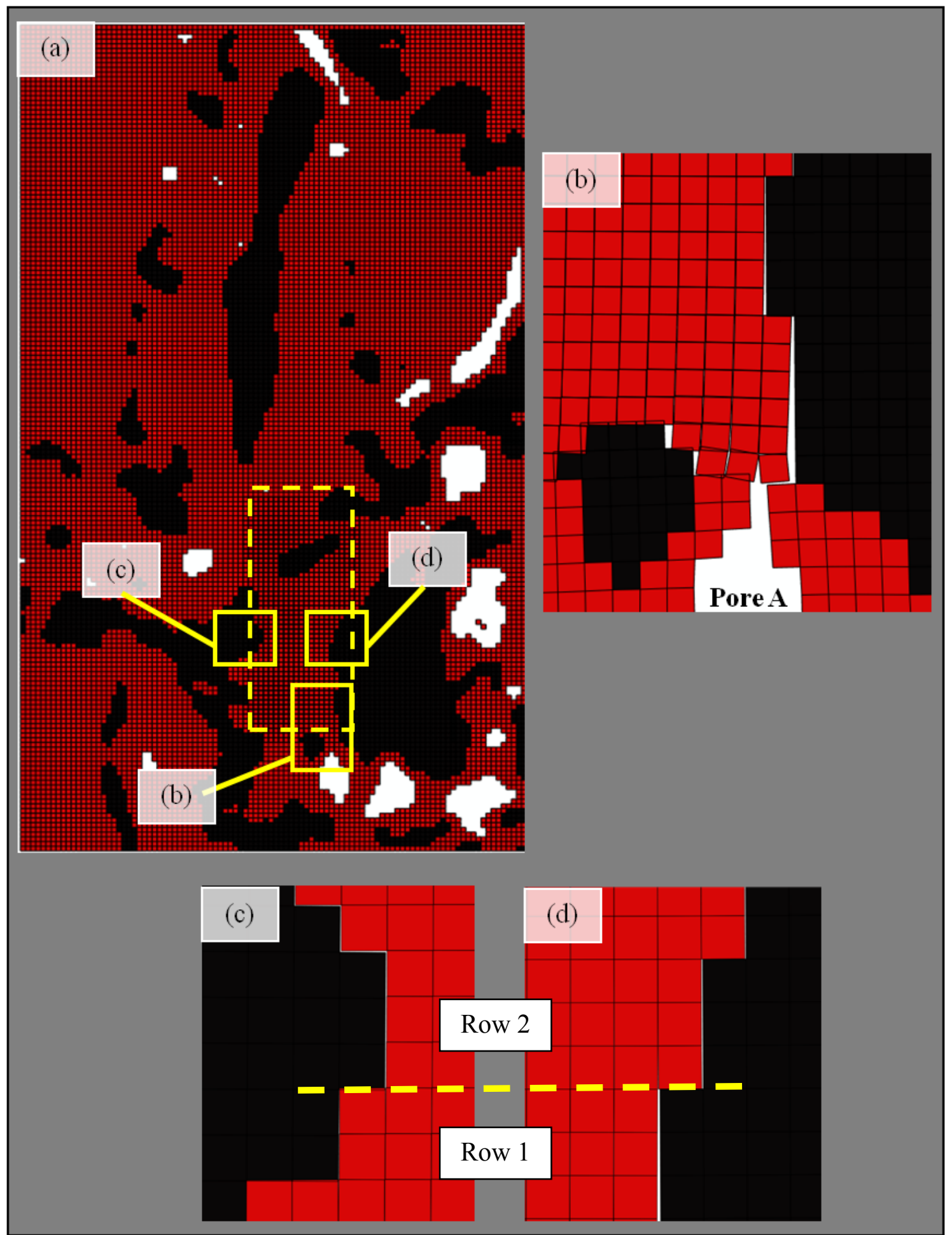

Figure 6-4: FE fracture simulation result with applied crystallization pressure: (a) The constructed FE model with fracture domain defined as highlighted in dotted line box. (b) An enlarged view of fracture zone above Pore A. (c) and (d) show the crack opening along the cement and clicker particle boundaries. 
The fracture behavior in the simulation was improved by introducing a predefined crack path based on the crack opening magnitude analysis in the simulation results, as illustrated in Figure 6-4(c) and (d). Figure 6-5(a) shows the outcome of the analysis with predefined crack path. Figure 6-5(b) and (c) demonstrate enlarged view of the crack path highlighted in yellow dotted box in Figure 6-5(a) that produced crack opening. The crack did not open outside the highlighted box (b). This can be explained by the strength of the applied pressure was insufficient to cause the crack to propagate further.

Thus, by assuming the crack generated in the highlighted section in Figure 6-5(b) turned into a pore, the fracture simulation was performed only on top half of the model with applied crystallization pore pressure in the Pore B (Figure 6-6). For an average radius of approximately $500 \mathrm{~nm}$, the crystallization pore pressure in the Pore B was equivalent to $8.84 \mathrm{MPa}$ (Equation (1-4)). The sub-cooling temperature was estimated to be approximately $-7.51^{\circ} \mathrm{C}$ (Equation (1-1)).

Multiple columns of bilinear CZM elements were first defined to analyze the crack opening magnitudes along the hydrated-unhydrated cement interface, as shown in Figure 6-6(a). Figure 6-6(b) illustrates the enlarged view of the highlighted section in Figure 6-6(a) that shows the crack opening on the interface. The analyzed result indicated that the crack would tend to grow in the left face of the unhydrated cement. Thus, a predefined crack was developed based on the analyzed result, as shown in Figure 6-6(c). Figure 6-6 (d) shows the enlarged view of crack opening with predefined crack path along the hydrated-unhydrated cement interface. Comparing to the crack path developed in the actual model, as shown in Figure 6-7, the predicted crack path in the bottom (Area B) and upper (Area A) halves showed a well agreement to the actual crack grow path. 
Thus, it is validated that the bilinear CZM technique is feasible to be carried out to perform internal frost damage modeling on cement paste samples.

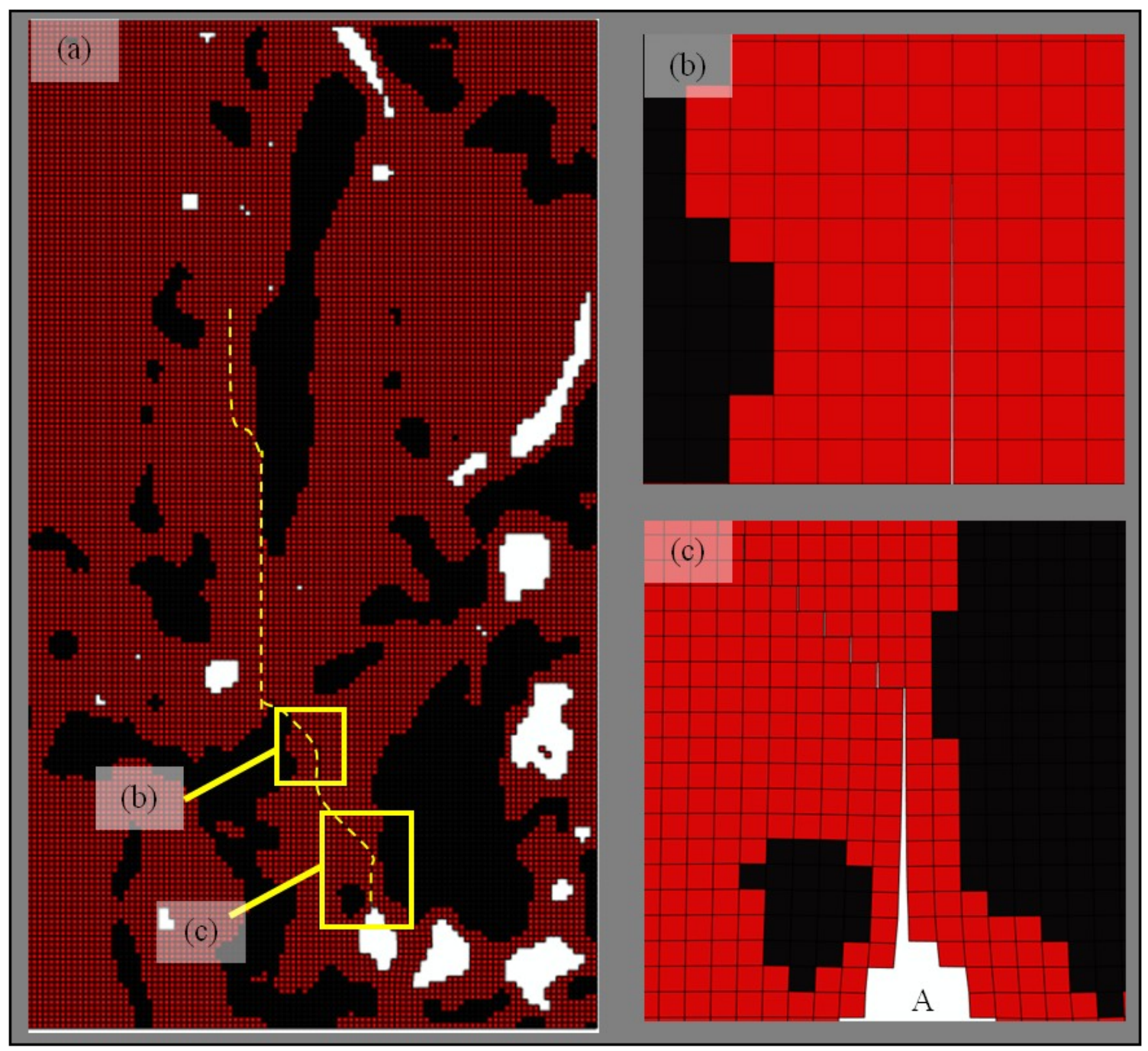

Figure 6-5: Bilinear CZM simulation result in ABAQUS on cement paste due to applied crystallization pressure: (a) The mesh constructed in ABAQUS with CZM boundary defined in yellow dotted line. (b) and (c) show the enlarged view crack opening in the highlighted area along the predefined crack path as a result of crystallization pressure on Pore A. 


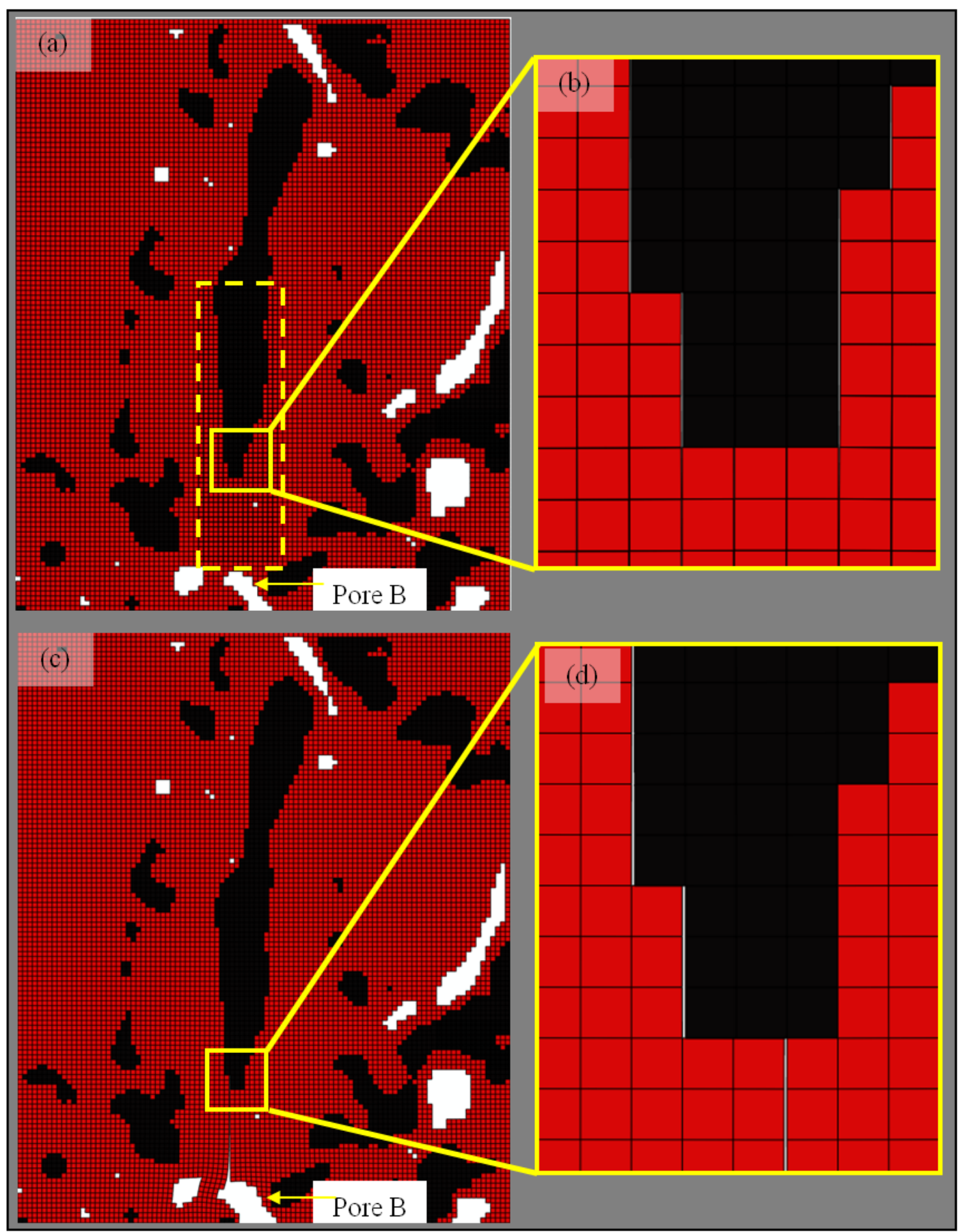

Figure 6-6: Bilinear CZM simulation result in ABAQUS on top half of the cement paste model: (a) The mesh constructed in ABAQUS with CZM boundary defined in the highlighted area (yellow dotted boundary). (b) An enlarged view that shows the crack opening along the hydrated-unhydrated cement interface. (c) A predefined crack growth path developed in the model with crack opening and (d) an enlarged view that shows the crack opening along the hydrated-unhydrated cement interface as a result of crystallization pressure from Pore B. 


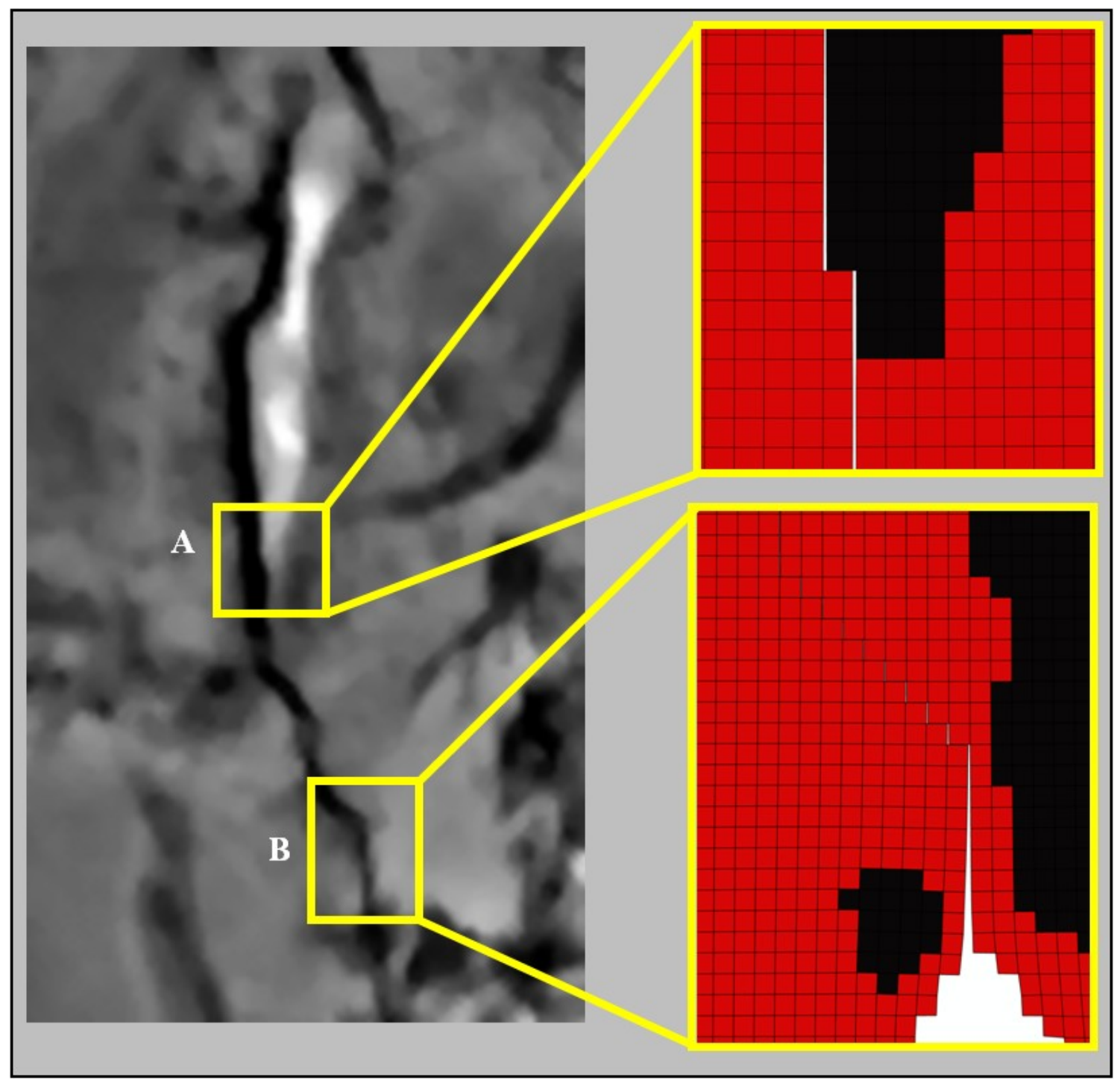

Figure 6-7: Validation of bilinear 2-D CZM fracture simulation result to the actual fracture sample. The highlighted area A and B show the simulated crack agreed well with the actual crack developed in the damaged cement paste sample.

\subsection{Computational Prediction of Internal Frost Damage with 3-D SEM Image Samples}

For a better visualization, a 3-D fracture simulation has been performed for the internal frost damage analysis on the cement paste sample. Since the SEM technique can only capture 2-D image, a 3-D image reconstruction was performed to generate a random 
microstructure of 3-D image by maintaining the porosity of the 2-D SEM sample. Due to random generation of 3-D microstructure, the 3-D simulation can only be performed on undamaged cement paste sample.

\subsubsection{3-D SEM digital sample generation}

An image of an undamaged cement paste sample had been selected for performing the 3-D fracture simulation, as shown in Figure 4-5(a). A section of $300 \times$ 300 pixels of the image was selected and cropped with a dimension of $0.096 \mu \mathrm{m} \times 0.096$ $\mu \mathrm{m}$ for each pixel. From the correlation function, the porosity was identified to be $6.35 \%$. The data was then applied for 3-D image reconstruction to generate a periodic microstructure. The initial generated 3-D image contained noise. The sintering algorithm was applied for noise reduction. At the end of sintering, a hydraulic radius of $5 \mu \mathrm{m}$ was defined to generate a 3-D microstructure image with reduced noise and the porosity remained as $6.35 \%$. The complete steps for the $3-\mathrm{D}$ image reconstruction and processing were shown in Figure 4-10 in Chapter 4 Section 4.2.3.3.

Figure 6-8 illustrates six image slices that were cropped from the reconstructed 3D image from slices 142 to 147 . Each image has a size of $60 \times 50$ pixels. All the image data were read by using the developed MATLAB scheme to construct FE mesh in the ABAQUS. Assuming the crack was initiated from the Pore A and extended to Pore B, a zigzag crack path with bilinear CZM element was constructed in each image model. All image slices were defined with the same crack path. The solid phase was defined with hydrated cement paste material properties, which are listed in Table 6-2. That is, the Young's modulus and Poisson's ratio are $22 \mathrm{GPa}$ and 0.25 , respectively. The bilinear 
CZM parameters were also defined as listed in Table 6-2. That is, for hydrated cement bonding, the fracture energy and tensile strength were $37.5 \mathrm{~J} / \mathrm{m}^{2}$ and $0.15 \mathrm{GPa}$, respectively.

All the surfaces of the 3-D model were defined with roller supports to create a confined boundary. For a pore radius of $5 \mu \mathrm{m}$ (based on the sintering algorithm), assumed a $5 \mathrm{~nm}$ capillary pore connected to this pore. The crystallization pore pressure was computed to be approximately $2 \mathrm{MPa}$ (Equation (1-4), when the ice bulges into the capillary pore at the sub-cooling temperature of $-16.67{ }^{\circ} \mathrm{C}$ (Equation (1-1)). The pore pressure was defined on the element surfaces in accordance to the ABAQUS scheme for using the *DSLOAD command, as presented in Chapter 5 Section 5.3.1.

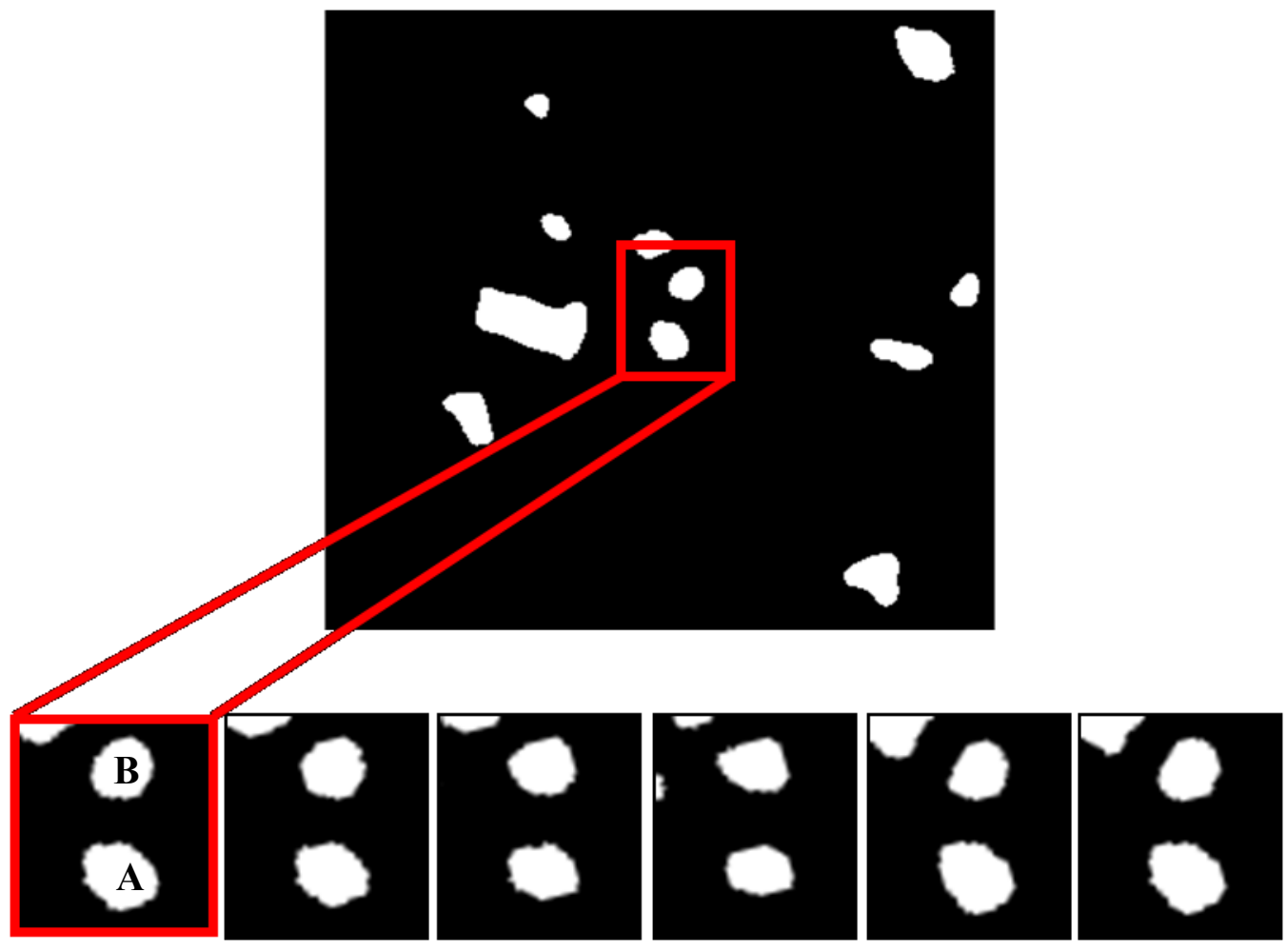

Figure 6-8: Image slices that contain pores were obtained from the reconstructed 3-D image of the cement paste sample with a w/c of 0.47 and a porosity of $6.35 \%$. 


\subsubsection{Computational simulation of internal frost damage with 3-D SEM digital samples}

Figure 6-9 shows the developed 3-D FE model in the ABAQUS from the six image slices (as shown in Figure 6-8) constructed in the developed MATLAB scheme. Figure 6-9 (b) also shows that the crack opening as a result of crystallization pore pressure of $20 \mathrm{MPa}$ induced in the Pore $\mathrm{A}$, and the crack extended to Pore $\mathrm{B}$ at the temperature of approximately $16.67{ }^{\circ} \mathrm{C}$. The result indicated that the computed crystallization pore pressure was sufficient to cause damages to the cement paste through the thermodynamic principle in a 3-D model. Thus, it is feasible to perform internal frost damage analysis on 3-D image for the cement paste sample based on the computed crystallization pore pressure. 
(a)

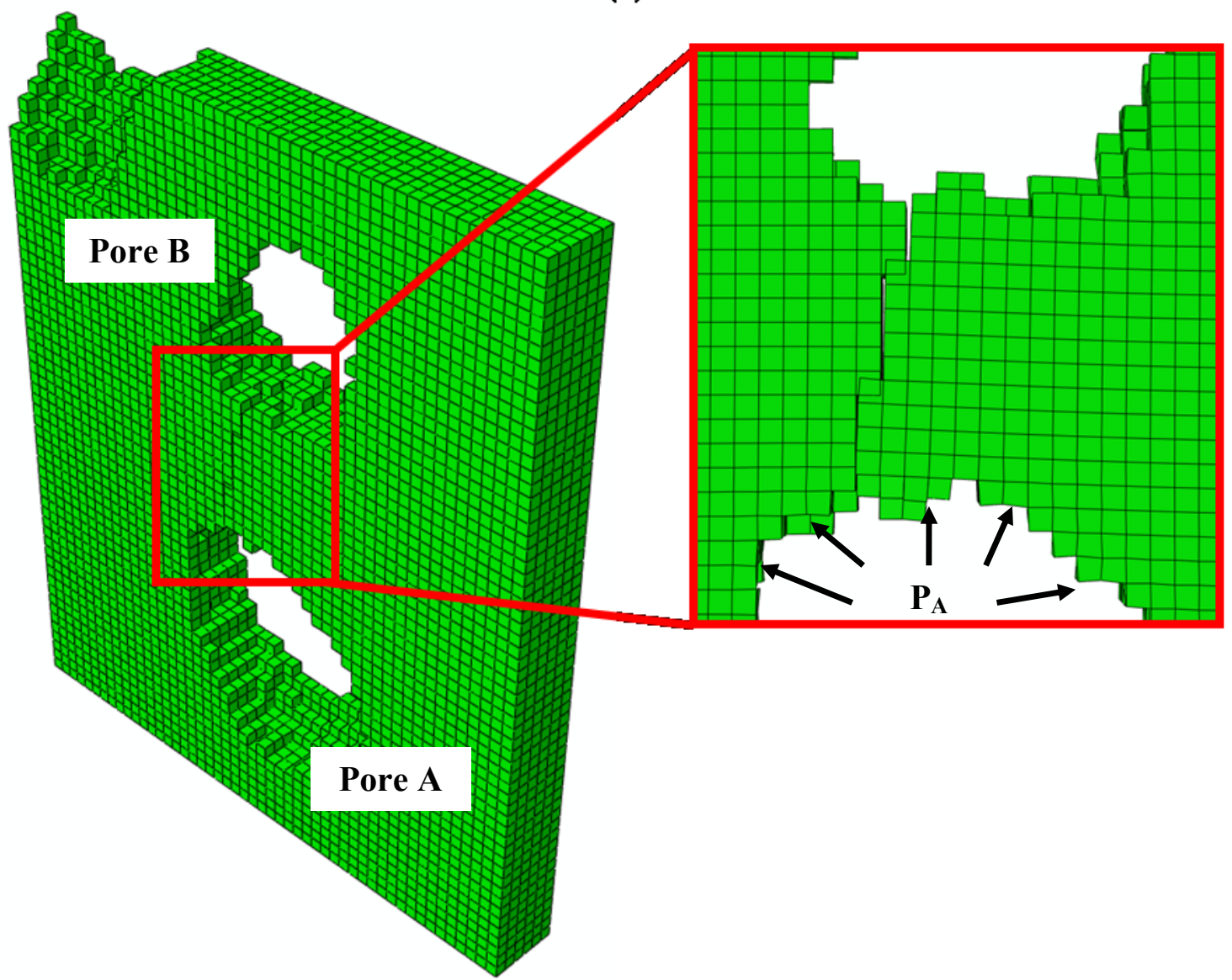

Figure 6-9: 3-D fracture simulation of internal frost damage analysis on reconstructed 3$\mathrm{D}$ image for the cement paste sample with a $\mathrm{w} / \mathrm{c}$ of 0.47 and a porosity of $6.35 \%$. The highlighted area display the crack developed as a result crystallization pressure effect.

\subsection{Computational Prediction of Internal Frost Damage with 3-D TXM Image Samples}

Since the SEM image has the limitation to validate the 3-D simulation result to the actual image sample, the TXM image of damaged cement paste sample was utilized here for validation purpose. The cement paste sample had a w/c of 0.45 and was damaged after performing 60 freeze-thaw cycles. Comparing to the SEM images, TXM images had 
a resolution of $30 \mathrm{~nm} \times 30 \mathrm{~nm}$, which met this doctoral proposal goal to study internal frost damage analysis in the nano-pores.

\subsubsection{3-D TXM image processing and digital sample generation}

The TXM image of a freeze-thaw damaged cement paste sample with w/c of 0.47 was selected to validate the internal frost damage analysis through the bilinear CZM technique in ABAQUS. Figure 6-10 demonstrates the area of interest (yellow box) that was selected for validating simulation result from the TXM image slice 4 . The area was selected as a developed crack was observed from slices 1 to 10 from the TXM image. The images were then cropped to a dimension of $80 \times 130$ pixels followed by keeping image slices from 1 to 6 for performing 3-D fracture simulation. These images were kept as they showed a clear developed crack path.

Figure 6-11(a) shows the six image slices cropped from the cement paste TXM image slices. The images were oriented $180^{\circ}$ as the developed bilinear CZM mesh scheme was only capable of generating pressure from the bottom half of the images. The images showed that the crack was initiated from Pore A and was propagated and approaching to Pore B. The noise in the images was then reduced by applying the median filter technique with a radius of 2.0 pixels by using the ImageJ. Figure 6-11(b) shows the resulting image slices after performing noise reduction through filtering technique. The images were read into the developed MATLAB code for constructing FE mesh with bilinear CZM elements defined along the predefined crack path. Each element was equivalent to a pixel size of the image, which had a dimension of $30 \mathrm{~nm} \times 30 \mathrm{~nm}$. Each

element was also defined with a thickness of 1 unit length or $30 \mathrm{~nm}$. Two phases were 
constructed for the bilinear CZM fracture model, they were pore $(<60)$ and solid phases $(\geq 60)$.

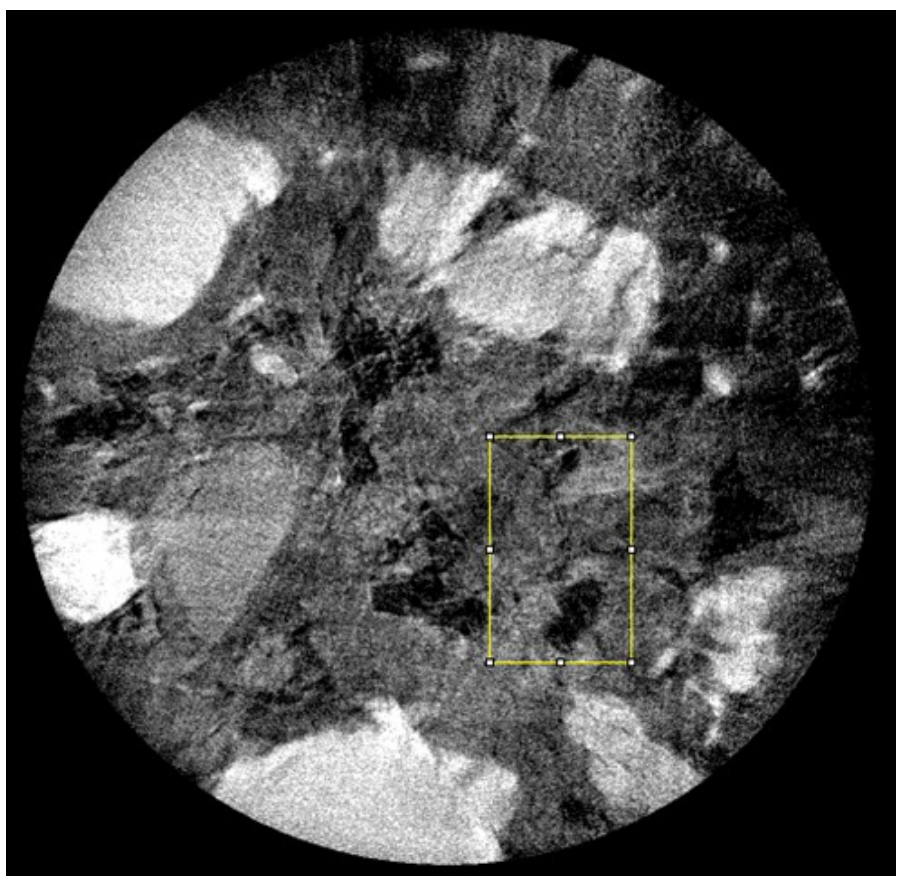

Figure 6-10: One of the TXM image slices that showed a crack pattern in the highlighted box in ImageJ (http://rsb.info.nih.gov/ij/). 

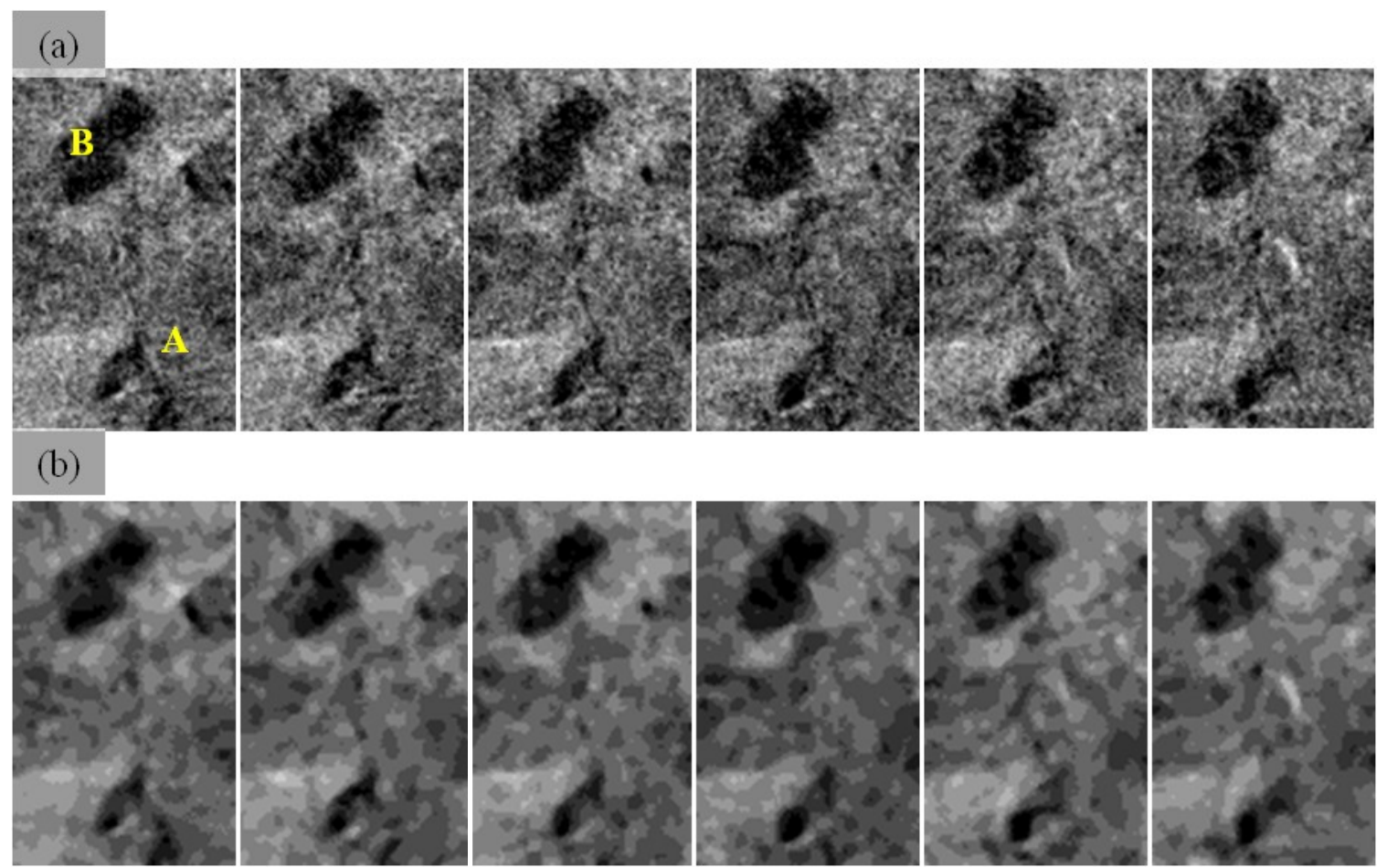

Figure 6-11: The six selected TXM image slices that displayed crack path: (a) The actual image samples before noise removal and (b) the filtered images with noises removed.

The solid phase of this model includes hydrated cement phase and unhydrated cement particles. The material properties were defined with Young's modulus and Poisson's ratio equivalent to $22 \mathrm{GPa}$ and 0.25 (as listed in Table 6-2), respectively. A zigzag crack path that emulated to the actual developed crack path in the damaged cement paste sample was defined in the bilinear CZM fracture model. The approach was to verify the damage was caused by crystallization pore pressure from the Pore A through the crack growth path. The bilinear CZM parameters for the hydrated cement phase were defined with fracture energy and tensile strength of $37.5 \mathrm{~J} / \mathrm{m}^{2}$ and $0.15 \mathrm{GPa}$ (as listed in Table 6-2), respectively. For boundary conditions, roller supports were defined on all six 
faces of the 3-D image. And a distributed pressure was defined inside the Pore A surfaces by using the *DSLOAD command based on the ABAQUS scheme, as presented in the Chapter 5 Section 5.3.1. With the pore size was approximately $500 \mathrm{~nm}$ in radius (through calculating the number of elements), assumed a connected capillary pore of $5 \mathrm{~nm}$ in radius. The crystallization pore pressure of $20 \mathrm{MPa}$ inside of this pore was computed (Equation (1-4) when the ice bulged into the capillary pore by reaching a freezing temperature of $-17^{\circ} \mathrm{C}$ (Equation (1-1)), according to thermodynamic theory.

\subsubsection{Computational simulation and validation of internal frost damage with 3-D TXM digital samples}

Figure 6-12 (a) shows the FE mesh constructed based on the TXM image slices data. The crack pattern (as highlighted in yellow dotted line) developed in the actual damaged cement paste model was emulated and defined in the FE model with the bilinear CZM elements. Figure 6-12 (b) shows one of the TXM image slices of the damaged cement paste sample with highlighted crack path in yellow dotted line. Figure 6-12 (c), (d) and (e) shows the enlarged view of the fracture zone in the simulated FE model. The results showed the crack opening as a result of crystallization pore pressure applied in the Pore A and were well matched with the actual damaged cement paste. Thus, the crack path developed in the damaged cement paste model was validated by the computational simulation results of the internal frost damage analysis based on the thermodynamic principles. 
(a)

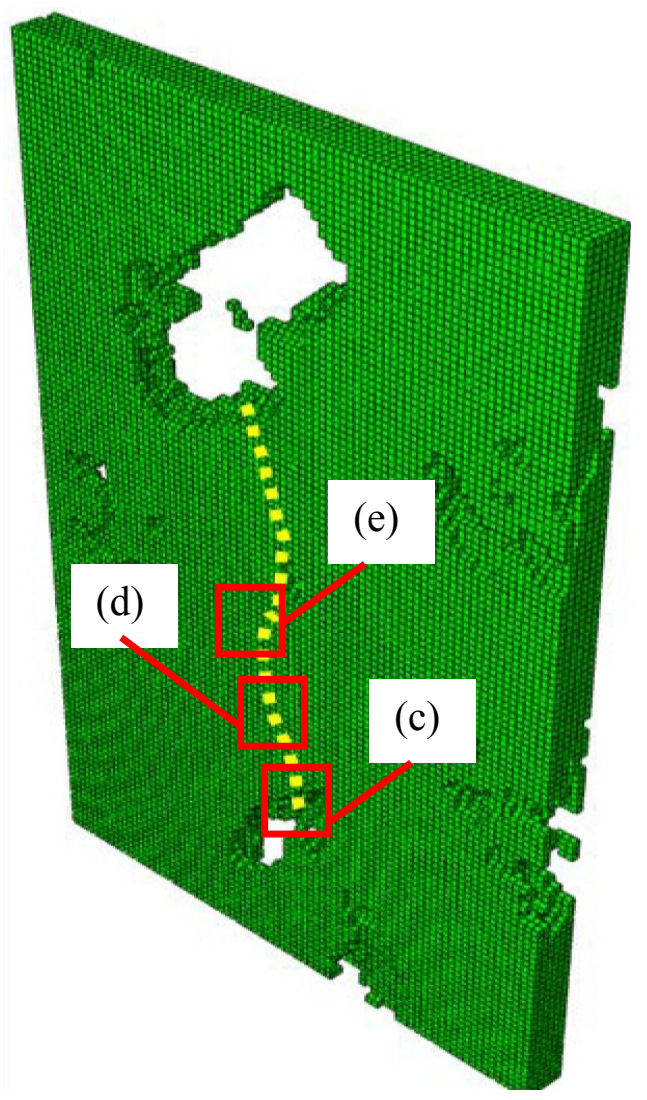

(b)
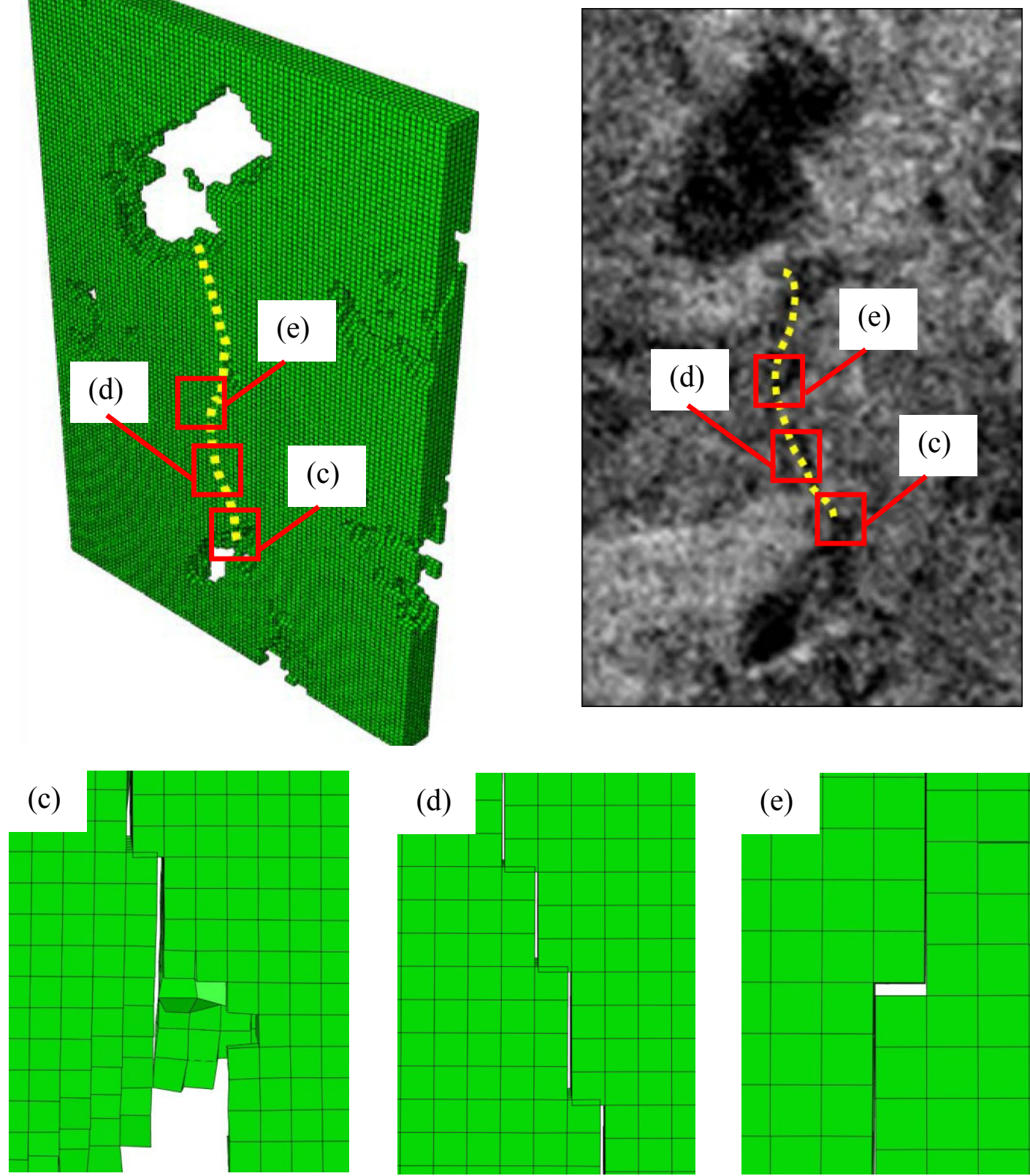

\begin{abstract}
(d)
\end{abstract}

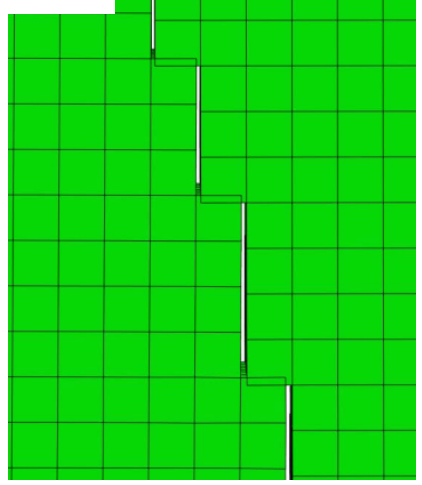

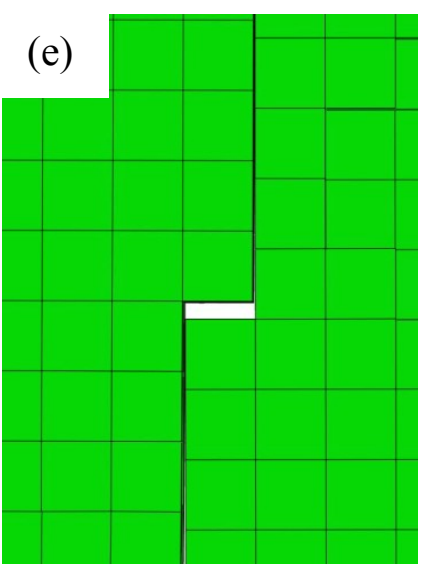

Figure 6-12: Validation of the fracture simulation by comparing the damaged cement paste sample with a w/c $=0.47$ : (a) The constructed FE model based on the TXM image slices of the cement paste sample with predefined crack path (bilinear CZM elements) as indicated in dotted yellow line. (b) One of the slice images with actual crack path as highlighted in yellow dotted line. (c), (d) and (e) The enlarged view of the crack opening in the FE model as a result of the crystallization pore pressure. The damages on the cement paste sample were validated by the computational simulation results. 


\subsection{Concluding Remarks}

This doctoral study applied XFEM and bilinear CZM techniques to numerically investigate micro-scale damage behavior within digital samples of cement-based materials under various loading conditions. The SEB bending test on a concrete specimen was firstly performed for validation. The SEB simulation was conducted on concrete digital samples generated from millimeter-scale images. For XFEM technique, the search distance and kink angle approach was employed to predict the crack growth path based on the highest energy released rate. For bilinear CZM, three types of cohesive/adhesive fracture behavior were defined for cement and aggregate phases and interfacial zone in the computational model. The predicted crack paths from both XFEM and bilinear CZM models show the damage pattern that is very close to the crack evolution on the tested concrete specimen. The results indicated that both techniques can be employed to perform fracture simulations on cement-based materials.

With the successful results obtained from the SEB bending test simulation results, the internal frost damage analysis was then performed through 2-D and 3-D fracture simulations. The multiphase 2-D and 3-D bilinear CZM models were developed to account for the internal cohesive and interfacial adhesive fracture behavior of cementbased materials. To capture microstructure of cement paste samples, the SEM and TXM imaging techniques were applied to characterize the material phases and generate digital samples for fracture simulation.

The digital cement samples were generated from SEM image in tenths of micron scale. The pores, hydrated cement and unhydrated cement particles were identified. A CT 
test fracture model was simulated to validate the capability of the bilinear CZM technique on modeling fracture behavior in a porous media. The CT test was simulated along a predefined crack path within one selected porous cement sample. It showed that the coalescing occurred in the porous system from the digital image sample. Thus, it is feasible to perform fracture simulations on porous cement-based sample by using the bilinear CZM technique.

The internal frost damage is mainly caused by ice crystallization pressure in pores. The simulation study was conducted by analyzing the crystallization pore pressure by assuming that an idealized spherical pore was connected with a cylindrical capillary pore channel. The magnitude of the pressure loading and the sub-cooling temperature were computed based on thermodynamic principles. The pressure loading were applied in the developed 2-D and 3-D models and crack opening was observed in the model due to the internal frost damage.

Following this, the crack propagation was also simulated within an actual cement paste sample from SEM digital image by considering cohesive fracture behaviors with hydrated cement, unhydrated cement particles and interfacial zones. The bilinear CZM model predicted the frost damage evolution that was compared favorably with captured crack path in the SEM digital sample. Overall, the simulation study showed that the multiphase bilinear CZM model can reasonably predict the crack nucleation and propagation with microstructure systems of cement and concrete.

The 3-D fracture simulations were also performed for the internal frost damage analysis. The SEM reconstructed 3-D image of undamaged cement paste microstructure was firstly performed to determine the capability of the developed bilinear CZM 
technique to simulate fracture process in a 3-D model. The successful crack opening generation from the simulation result allowed the validation of fracture analysis on the freeze-thawed cement paste sample to be carried out. The image of the damaged cement paste sample was acquired from TXM machine. The TXM image slices which have a resolution of $30 \mathrm{~nm} \times 30 \mathrm{~nm}$ had captured the crack developed from pores. The damages were expected to be caused by the induced crystallization pore pressure in the subcooling temperature. A bilinear CZM fracture model was developed based on the six TXM image slices. This means the physical thickness of the six slices is $180 \mathrm{~nm}$. The computed crystallization pore pressure based on the thermodynamic principle was applied on the pore where the crack initiation was observed in the actual damaged sample. The crack path that emulated the actual developed crack path had shown that the crack opening as a result of the applied pressure. The result had validated that the internal frost damage caused by ice crystallization pressure occurs within pores of cement-based materials under sub-cooling temperatures. 


\section{CHAPTER 7 CONCLUSIONS AND FUTURE WORKS}

\subsection{Summary of Research Investigations}

Some theories suggested that the internal frost damage is the main factor that causes damages and deterioration on concrete pavement. However, there are no supportive experimental evidences and computational simulations that prove the crystallization pore pressure can damage cementitious materials under subcooling temperatures. Further investigation are needed by capturing microscale damage evolution through imaging data acquisition on freeze-thaw tested samples, microstructure-based modeling based on thermodynamic principles, and experimental characterization. With these motivations, the objective of this doctoral study is to investigate the internal frost damage due to crystallization pore pressure in porous cement-based materials by developing computational and experimental characterization tools.

The ultimate goal of this study is to support the theory of internal frost damage mechanism on concrete by integration of computational modeling and experimental tests. The computatioanl tools were developed to simulate internal frost damage evolution by applying computed crystallization pore pressure based on thermodynamic principles. The experimental characterization tests were conducted to capture damage evolution within capillary pore structure in micro and nano scales. In order to achieve this final goal, four tasks were implemented to develop micromechanical analysis models and experimental characterization methods for studying internal frost damage in cementitious materials.

1. Task 1: Development and validation of computational fracture models 
2. Task 2: Pore pressure calculation and damage simulation on idealized samples

3. Task 3: Conducting controlled freeze-thaw test and microstructure image acquisition

4. Task 4: Numerical validation of crystallization pressure mechanism for frostinduced damage in cement samples.

It is essential to develop integrated computational and experimental tools to achieve the study objective. Thus, task 1 was firstly performed in order to develop and validate computational fracture models. In order to determine the feasibility of numerical tools on performing fracture simulations, both stress intensity factor (SIF) and crack growth trend were utilized. Compact tension (CT) test and single-edge notched beam (SEB) bending test on concrete sample were developed. Both extended finite element method (XFEM) and bilinear cohesive zone modeling (CZM) techniques were employed as the numerical tools. The relatively low error percentage observed in the XFEM CT and SEB models indicated the XFEM tools can predict fracture behavior on concrete. In addition, the crack growth paths predicted with the XFEM and bilinear CZM models were compared well with the actual tested sample. These results indicated that both XFEM and bilinear CZM techniques can be employed to develop fracture modeling on concrete.

With the availability of numerical tools to perform fraction simulations on concrete obtained in Task 1, the internal frost damage analysis can be done on numerical models. Thus, Task 2 was then carried out to calculate the crystallization pore pressure based on thermodynamic principles. The computed pressures were input into the FE models by utilizing the XFEM and bilinear CZM techniques on idealized pore system of 
concrete. The computed pressures on different pore geometries were found to be sufficient to cause cracks to propagate and open in the FE models. Thus, the results supported the theory of internal frost damage that can cause damages on concrete as a result of crystallization pore pressure. Both numerical and computational analyses were also applied in Task 4 to validate the internal frost damage based on image samples that were obtained in Task 3.

Computational analysis results from Task 2 on idealized samples were not sufficient to support the theory of internal frost damage on concrete. Thus, Task 3 was carried out to fabricate samples and conducting controlled freeze test followed by acquiring microstructure image. Cement paste samples with high w/c (i.e. w/c $=0.45$ and 0.47) were prepared in millimeter and micron meter scales and controlled freeze-thaw tests were then performed. The microstructure images of tested samples were observed through scanning electron microscope (SEM) and transmission X-ray microscopy (TXM) in micro and nano scales respectively. Crack developments within pore systems were observed from the acquired microstructure images. Since the SEM samples have dimensions in millimeter, the shrinkage damage introduced during SEM sample preparation was significantly reduced. While for the TXM imaging of freeze-thaw tested sample, it was expected that the damages were caused by the crystallization pore pressure.

Finally, Task 4 was carried out to perform micro-damage model validation by incorporating the numerical tools developed in Task 1, the pore pressure calculation approach in Task 2, and acquired microstructure image of cement paste sample in Task 3. The acquired microstructure images from damaged cement paste samples were utilized to 
perform fracture modeling with computed crystallization pressure on specific pores. All the fracture modeling results had shown crack opening as a result of the crystallization pore pressure that were computed based on the thermodynamic principles. Besides, the simulation results had shown that the crack opened along the predicted crack path similar to the actual sample image. Thus, the results not only indicated that the internal frost damage model can be simulated, but also validated that the actual developed crack in the damaged cement paste sample was the result of crystallization pore pressure effect from microstructural aspects.

To conclude, the computed crystallization pore pressures based on the thermodynamic principles were sufficient to induce damages on concrete in the subcooling temperature. The results verified that the internal frost damage can lead to damages on concrete pavement. With these successful results and investigations, this doctoral study has contributed to the whole knowledge of concrete by enhancing the knowledge of frost damage in concrete through thermodynamic analysis and integrated micromechanical modeling development and experimental tests. In addition, this doctoral study has also created a breakthrough on the experimental microstructure characterization in micro and nano scales by utilizing advanced technical instruments such as SEM and TXM.

\subsection{Suggestions for Future Work}

Even though the research has been completed, some new ideas were provided based on the current available resources. As a result of limited time, the ideas can be 
implemented for the future works. The ideas will not only improve the modeling work but also create some research opportunities in other areas.

\subsubsection{In-situ TXM investigation of freeze-thaw damage of cement paste specimens}

In this doctoral study, the nano-scale cement paste microstructure was successfully acquired by using the TXM machine. The cracks were also observed on the freeze-thawed damage sample from the TXM image slices. Since it is the first time that the APS facility dealt with cement paste samples in micron scale, it took some time and effort on calibrating the TXM machine. As a result, the in-situ TXM investigation of freeze-thaw damage could not be perform as planned on the cement paste specimens. Besides, applying for using the TXM machine is very competitive due to its advantages of fast-image acquisition and high image resolutions capturing. In this doctoral study, a total of three cycles were applied and only the third cycle application was granted for using the TXM machine. With the successful results and experiences, the in-situ freezethaw damage investigation can be carried out in the future with more samples that are made of various mixture designs. In addition, the publication of TXM results will ease the TXM usage application in the future.

\subsubsection{Employing XFEM technique for fracture simulation in a multi-pore system}

This doctoral study had shown the capability of the developed XFEM program to simulate crack growth in the cement-based material model. However, modeling coalescing of pores is the only limitation of this current developed XFEM program. In this doctoral study, the XFEM program had been altered to improve the performance on 
simulating the crack growth in the heterogeneous media with elliptical inclusions. Thus, the author believes that there is still a lot of freedom of alteration in the levelset function to be carried out to overcome the limitation.

\subsubsection{Generating predicted crack growth path from acquired image data}

The successful simulation results in this doctoral study had displayed that the crack tends to develop along the matrix-aggregate or hydrated-unhydrated cement interface. Thus, it is feasible to develop a new scheme in the existing developed MATLAB program to generate predicted crack growth path from the image data. The search distance and the kink angle schemes can be employed to detect for the highest energy released rate or other fracture criterion in order to predict the next crack growth direction.

\subsubsection{In-situ micromechanical behavior investigation on cement paste sample}

The successful 3-D image data acquisition of damaged cement paste sample by using TXM machine in this doctoral study has broaden the aspect of studying cementbased material microstructures. The fast image acquisition and fine resolutions (nano scale) allows the real time investigation of microstructure behavior. Hydration, fracture, and self-healing processes are among the aspects that can utilize the non-destructive technique to observe the in-situ microstructures behavior in 3-D in nano-scale level. Through the investigations, new mixture designs and damage preventions can be implemented to improve performance of concrete. 


\subsubsection{Statistical analysis on mixture design for concrete pavement}

From this doctoral study, the thermodynamic principle has determined that the lower the freezing temperature, the higher the crystallization pore pressure that can be induced on cement-based material. Thus, by gathering weather data from the region of interest, a statistical analysis can be performed on mixture design for concrete pavement. This can be done through optimization, which considered other factors, such as the loading and traffic rates, for design a durable and sustainable concrete pavement in the northern region of America. 


\section{REFERENCES}

ABAQUS. (2004). In: Hibitt, Karlsson and Sorenson, Version 6.5, Pawtucket, R.I.

Abdelaziz, Y., and Hamouine, A. (2008). "A survey of the extended finite element." Computers \& Structures, 86(11-12), 1141-1151.

Al-Omari, A., and Masad, E. (2004). "Three dimensional simulation of fluid flow in Xray CT images of porous media." International Journal for Numerical and Analytical Methods in Geomechanics, 28(13), 1327-1360.

Anderson, T. L. (1995). Fracture Mechanics: Fundamentals and Applications, 2nd Ed., C.R.C Press, Inc.

ASTM. (2009). "Annuaal Book of ASTM Standards: Concrete and Aggregates." In: Section Four: Construction.

Barenblatt, G. I. (1959). "The formation of equilibrium cracks during brittle fracture. General ideas and hypotheses. Axially-symmetric cracks." Journal of Applied Mathematics and Mechanics, 23(3), 622-636.

Bazant, Z. P., and Pijaudier-Cabot, G. (1988). "Nonlocal Continuum Damage, Localization Instability and Convergence." Journal of Applied Mechanics, 55(2), 287-293.

Belytschko, T., and Black, T. (1999). "Elastic crack growth in finite elements with minimal remeshing." International Journal for Numerical Methods in Engineering, 45(5), 601-620.

Belytschko, T., Lu, Y. Y., and Gu, L. (1994). "Element-free Galerkin methods." International Journal for Numerical Methods in Engineering, 37(2), 229-256.

Bentz, D., Quenard, D., Kunzel, H., Baruchel, J., Peyrin, F., Martys, N., and Garboczi, E. (2000a). "Microstructure and transport properties of porous building materials. II: Three-dimensional X-ray tomographic studies." Materials and Structures, 33(3), $147-153$.

Bentz, D. P., Garboczi, E. J., and Quenard, D. A. (1998). "Modelling drying shrinkage in reconstructed porous materials: application to porous Vycor glass." Modelling and Simulation in Materials Science and Engineering, 6(3), 211.

Bentz, D. P., and Martys, N. S. (1994). "Hydraulic radius and transport in reconstructed model three-dimensional porous media." Transport in Porous Media, 17(3), 221238. 
Bentz, D. P., Pimienta, P. J. P., Garboczi, E. J., and Carter, W. C. (1991). "Cellular Automation Simulations of Surface Mass Transport Due to Curvature Gradients: Simulations of Sintering in 3-D." MRS Proceedings, 249(413), doi:10.1557/PROC-1249-1413

Bentz, D. P., Quenard, D. A., Kunzel, H. M., Baruchel, J., Peyrin, F., Martys, N. S., and Garboczi, E. J. (2000b). "Microstructure and transport properties of porous building materials. II: Three-dimensional X-ray tomographic studies." Materials and Structures/Materiaux et Constructions, 33(227), 147-153.

Berryman, J. G. (1985). "Measurement of spatial correlation functions using image processing techniques." Journal of Applied Physics, 57(7), 2374-2384.

Brandt, A. M. (1995). Cement Based Composites: Materials, Mechanical Properties, and Performance Chapman \& Hall, London, UK.

Brun, M., Lallemand, A., Quinson, J.-F., and Eyraud, C. (1977). "A new method for the simultaneous determination of the size and shape of pores: the thermoporometry." Thermochimica Acta, 21(1), 59-88.

Bush, M. B. (1997). "The Interaction between a Crack and a Particle Cluster." International Journal of Fracture, 88(3), 215-232.

Buttlar, W. G., Paulino, G. H., and Song, S. H. (2006). "Application of graded finite elements for asphalt pavements." Journal of Engineering Mechanics, 132(3), 240249.

Chen, Y.-c. K., Chu, Y. S., Yi, J., McNulty, I., Shen, Q., Voorhees, P. W., and Dunand, D. C. (2010). "Morphological and topological analysis of coarsened nanoporous gold by x-ray nanotomography." Applied Physics Letters, 96(4), 043122.

Cook, R. D. (1995). Finite element modeling for stress analysis, Wiley.

Cruse, T. (1988). Boundary element analysis in computational fracture mechanics, Kluwer: Dordrecht.

Dai, Q. (2010). "Micromechanical viscoelasto-plastic models and finite element implementation for rate-independent and rate-dependent permanent deformation of stone-based materials." International Journal for Numerical and Analytical Methods in Geomechanics, 34(13), 1321-1345.

Dai, Q. (2011). "Two- and three-dimensional micromechanical viscoelastic finite element modeling of stone-based materials with X-ray computed tomography images." Construction and Building Materials, 25(2), 1102-1114.

Dai, Q., and Ng, K. (2010). "An Extended Finite Element Model for Characterization of Concrete Fracture Properties with Compact Tension Tests." In: ASCE 
Engineering Mechanics Special Publication (EMSP) entitled "Pavements and Materials: Testing and Modeling in Multiple Length Scales, March.

Dai, Q., Ng, K., Zhou, J., Kreiger, E. L., and Ahlborn, T. M. (2012). "Damage investigation of single-edge notched beam tests with normal strength concrete and ultra high performance concrete specimens using acoustic emission techniques." Construction and Building Materials, 31(0), 231-242.

Dai, Q., and Sadd, M. H. (2004). "Parametric model study of microstructure effects on damage behavior of asphalt samples." International Journal of Pavement Engineering, 5(1), 19-30.

Dai, Q., Sadd, M. H., Parameswaran, V., and Shukla, A. (2005). "Prediction of damage behaviors in asphalt materials using a micromechanical finite-element model and image analysis." Journal of Engineering Mechanics, 131(7), 668-677.

Dai, Q., and You, Z. (2005). "Prediction of Creep Stiffness of Asphalt Mixture with Micromechanical Viscoelastic Finite Element Modeling." In: Eighth U.S. National Congress on Computational Mechanics (USNCCM8), Austin, Texas.

Dai, Q., and You, Z. (2007a). "Micromechanical Finite Element Framework for Predicting Viscoelastic Properties of Asphalt Mixtures." Materials and Structures, Springer Netherlands, Online at http://www.springerlink.com/content/6272035711512866

Dai, Q., and You, Z. (2007b). "Prediction of Creep Stiffness of Asphalt Mixture with Micromechanical Finite-Element and Discrete-Element Models." Journal of Engineering Mechanics, 133(2), 163-173.

Dai, Q., You, Z., and Sadd, M. H. (2006). "A Micromechanical Viscoelaso-Plastic Model For Asphalt Mixtures." In: Geotechnical Special Publication (GSP): Asphalt Concrete: Simulation, Modeling, and Experimental Characterization. American Society of Civil Engineers (ASCE) 12-20.

Dai, Q., Yu, X., Ng, K., and Liu, Z. (2011). "Development of Micromechanics Models and Innovative Sensor Technologies to Evaluate Internal-Frost Damage of Concrete." Journal of the Transportation Research Board, National Academies, 2240, 50-58.

Dowling, N. E. (2007). Mechanics Behavior of Materials: Engineering Methods for Deformation, Fracture, and Fatigue, Third Edition Ed., Pearson International Edition.

Einstein, A. (1906). "Eine neue Bestimmung der Moleküldimensionen." Annalen der Physik, 324(2), 289-306. 
Espinosa, H. D., and Zavattieri, P. D. (2003). "A grain level model for the study of failure initiation and evolution in polycrystalline brittle materials. Part I: Theory and numerical implementation." Mechanics of Materials, 35(3-6), 333-364.

Fan, S. C., Liu, X., and Lee, C. K. (2004). "Enriched partition-of-unity finite element method for stress intensity factors at crack tips." Computers \& Structures, 82(45), 445-461.

Garboczi, E. J., Bentz, D. P., and Martys, N. S. (1999). "1. Digital Images and Computer Modeling." In: Experimental Methods in the Physical Sciences, W. Po-zen, ed., Academic Press, 1-41.

Guddati, M. N., Feng, Z., and Kim, R. (2002). "Toward a micromechanics-based procedure to characterize fatigue performance of asphalt concrete." Transportation Research Record(1789), 121-128.

Hasan, M., Ueda, T., and Sato, Y. (2008). "Stress-Strain Relationship of Frost-Damaged Concrete Subjected to Fatigue Loading." American Society of Civil Engineers, pp $37-45$.

Hsu, P.-C., Chu, Y., Yi, J.-M., Wang, C.-L., Wu, S.-R., Hwu, Y., and Margaritondo, G. (2010). "Dynamical growth behavior of copper clusters during electrodeposition." Applied Physics Letters, 97(3), 033101.

http://rsb.info.nih.gov/ij/. "ImageJ."

http://www.aps.anl.gov/Users/Prospective/. "Advanced Photon Source."

http://www.cement.org/pavements/pv cp highways.asp. (2012). "Portland Cement Association (PCA)." (3/27/2012.

http://www.ctlab.geo.utexas.edu/eng/index.php. "High Resolution X-ray CT Facility."

http://www.pa.pavement.com/why.htm. (2010). "American Concrete Pavement Association Pennsylvania Chapter." (3/27/2012.

Jacobsen, S., Marchand, J., and Hornain, H. (1995). "Sem observations of the microstructure of frost deteriorated and self-healed concretes." Cement and Concrete Research, 25(8), 1781-1790.

Jeon, I., Kang, K.-J., and Im, S. (2008). "Stress intensities at the triple junction of a multilevel thin film package." Microelectronics Reliability, 48(5), 749-756.

Kaufmann, J. (2000). "Experimental identification of damage mechanisms in cementitious porous materials on phase transition of pore solution under frost deicing salt attack." EMPA (Swiss Federal Laboratories for Materials Testing and Research) Publication(248), 7-177. 
Kaufmann, J. P. (2004). "Experimental identification of ice formation in small concrete pores." Cement and Concrete Research, 34(8), 1421-1427.

Ketcham, R. A., and Carlson, W. D. (2001). "Acquisition, optimization and interpretation of X-ray computed tomographic imagery: applications to the geosciences." Computers \& Geosciences, 27(4), 381-400.

Kim, H., Wagoner, M., and Buttlar, W. (2009). "Micromechanical fracture modeling of asphalt concrete using a single-edge notched beam test." Materials and Structures, 42(5), 677-689.

Kim, H., Wagoner, M. P., and Buttlar, W. G. (2008). "Simulation of Fracture Behavior in Asphalt Concrete Using a Heterogeneous Cohesive Zone Discrete Element Model." Journal of Materials in Civil Engineering, 20(8), 552-563.

Kim, Y.-R., Allen, D. H., and Little, D. N. (2007). "Computational Constitutive Model for Predicting Nonlinear Viscoelastic Damage and Fracture Failure of Asphalt Concrete Mixtures." International Journal of Geomechanics, 7(2), 102-110.

Kyritsis, K., Hall, C., Bentz, D. P., Meller, N., and Wilson, M. A. (2009). "Relationship Between Engineering Properties, Mineralogy, and Microstructure in CementBased Hydroceramic Materials Cured at $200^{\circ}-350^{\circ} \mathrm{C}$." Journal of the American Ceramic Society, 92(3), 694-701.

Li, R., and Chudnovsky, A. (1993). "Variation of the energy release rate as a crack approaches and passes through an elastic inclusion." International Journal of Fracture, 67, 169-177.

Liang, Z. R., Fernandes, C. P., Magnani, F. S., and Philippi, P. C. (1998). "A reconstruction technique for three-dimensional porous media using image analysis and Fourier transforms." Journal of Petroleum Science and Engineering, 21(3-4), 273-283.

Litvan, G. G. (1975). "Phase transitions of adsorbates: VI, effect of deicing agents on the freezing of cement paste." Journal of the American Ceramic Society, 58(1-2), 2630 .

Liu, L., Ye, G., Schlangen, E., Chen, H., Qian, Z., Sun, W., and van Breugel, K. (2011). "Modeling of the internal damage of saturated cement paste due to ice crystallization pressure during freezing." Cement and Concrete Composites, $33(5), 562-571$.

Logan, D. L. (2007). A first course in the finite element method, Thomson.

Mamlouk, M. S., and Zaniewski, J. P. (2006). Materials for civil and construction engineering, Second Ed., Pearson Education, Inc., Upper Saddle River, NJ. 
Marusin, S. L. (1995). "Sample preparation - the key to SEM studies of failed concrete." Cement and Concrete Composites, 17(4), 311-318.

Mehta, P. K., and Monteiro, P. J. M. (2006). Concrete: Microstructure, Properties, and Materials, Third Ed., McGraw-Hill.

Melenk, J. M., and Babuska, I. (1996). "The partition of unity finite element method: Basic theory and applications." Computer Methods in Applied Mechanics and Engineering, 139(1-4), 289-314.

Moës, N., Cloirec, M., Cartraud, P., and Remacle, J. F. (2003). "A computational approach to handle complex microstructure geometries." Computer Methods in Applied Mechanics and Engineering, 192(28-30), 3163-3177.

Moës, N., Dolbow, J., and Belytschko, T. (1999). "A finite element method for crack growth without remeshing." International Journal for Numerical Methods in Engineering, 46(1), 131-150.

Mondal, P. (2008). "Nonomechanical Properties of Cementitious Materials," Northwestern University, Evanston, Illinois.

Mouret, M., Bascoul, A., and Escadeillas, G. (1999). "Microstructural features of concrete in relation to initial temperature--SEM and ESEM characterization." Cement and Concrete Research, 29(3), 369-375.

Ng, K., and Dai, Q. (2011). "Investigation of Fracture Behavior of Heterogeneous Infrastructure Materials with Extended-Finite-Element Method and Image Analysis." Journal of Materials in Civil Engineering, 23(12), 1662-1671.

Ng, K., and Dai, Q. (2012). "Tailored Extended Finite-Element Model for Predicting Crack Propagation and Fracture Properties within Idealized and Digital Cementitious Material Samples." Journal of Engineering Mechanics, 138(1), 89100.

Ortiz, M., and Pandolfi, A. (1999). "Finite-deformation irreversible cohesive elements for three-dimensional crack-propagation analysis." International Journal for Numerical Methods in Engineering, 44(9), 1267-1282.

Osher, S., and Sethian, J. A. (1988). "Fronts propagating with curvature-dependent speed: Algorithms based on Hamilton-Jacobi formulations." Journal of Computational Physics, 79(1), 12-49.

Pais, M. J., and Kim, N. H. (2009). "Modeling failure in composite materials with the extended finite element and level set methods." 50th AIAA/ASME/ASCE/AHS/ASC Structures, Structural Dynamics, and Materials Conference(AIAA 2009-2393). 
Panneerselvam, D., and Panoskaltsis, V. P. (2005). "Numerical Implementation of a Hyperelastic-Viscoplastic Damage Model for Asphalt Concrete Materials and Pavements." In: Asphalt Concrete, Simulation, Modeling, and Experimental Characterization, , E. Masad, V. P. Panoskaltsis, and L. B. Wang, eds., ASCE, 61-79.

Park, K., Paulino, G. H., and Roesler, J. (2010). "Cohesive fracture model for functionally graded fiber reinforced concrete." Cement and Concrete Research, 40(6), 956-965.

Paulino, G. H., Song, S. H., and Buttlar, W. G. (2004). "Cohesive Zone Modeling of Fracture in Asphalt Concrete." In: 5th International RILEM Conference RILEM Publication, Limoges, France, 63-70.

Pigeon, M., and Pleau, R. (1995). Durability of concrete in cold climates, E \& FN Spon.

Pimienta, P. J. P., Garboczi, E. J., and Craig Carter, W. (1992). "Cellular automaton algorithm for surface mass transport due to curvature gradients simulations of sintering." Computational Materials Science, 1(1), 63-77.

Powers, T. C. (1949). "The Air Requirement of Frost-Resistant Concrete." Proc. Highway Res. Board, 29, 184-211.

Powers, T. C. (1975). "Freezing effects in concrete." ACI(SP 47), 1-12.

Powers, T. C., and Helmuth, R. A. (1953). "Theory of Volume Changes in Hardened Portland Cement Paste during Freezing." Proc. Highway Res. Board, 32, 285-297.

Prandtl, L. (1933). "Ein Gedankenmodell für den Zerreißvorgang spröder Körper." ZAMM - Journal of Applied Mathematics and Mechanics / Zeitschrift für Angewandte Mathematik und Mechanik, 13(2), 129-133.

Quenard, D., Xu, K., Künzel, H., Bentz, D., and Martys, N. (1998). "Microstructure and transport properties of porous building materials." Materials and Structures, 31(5), 317-324.

Reuss, A. (1929). Zeitschrift für angewandte mathematik und mechanik, Berlin.

Roesler, J., Paulino, G. H., Park, K., and Gaedicke, C. (2007). "Concrete fracture prediction using bilinear softening." Cement and Concrete Composites, 29(4), 300-312.

Sadd, M. H., Dai, Q., and Parameswaran, V. (2004a). "Microstructural simulation of asphalt materials: Modeling and experimental studies." Journal of Materials in Civil Engineering, 16(2), 107-115. 
Sadd, M. H., Dai, Q., Parameswaran, V., and Shukla, A. (2004b). "Microstructural Simulation of Asphalt Materials: Modeling and Experimental Studies." Journal of Materials in Civil Engineering, ASCE, 16(2), 107-115.

Sadd, M. H., Dai, Q., Parameswaran, V., and Shukla, A. (2004c). "Simulation of Asphalt Materials Using a Finite Element Micromechanical Model with Damage Mechanics." Journal of Transportation Research Board, 1832, 86-95.

Sanford, R. J., ed. (2003). Principles of Fracture Mechanics, Prentice Hall.

Scherer, G., and Valenza, J. (2005). "Mechanisms of Frost Damage." In: Materials Science of Concrete, 209-246.

Scherer, G. W. (1999). "Crystallization in pores." Cement and Concrete Research, 29(8), 1347-1358.

Scherer, G. W., Valenza II, J. J., and Simmons, G. (2007). "New methods to measure liquid permeability in porous materials." Cement and Concrete Research, 37(3), 386-397.

Shet, C., and Chandra, N. (2002). "Analysis of energy balance when using cohesive zone models to simulate fracture processes." Journal of engineering materials and technology, 124(4), 440-450.

Soares, J., de Freitas, F., and Allen, D. (2003). "Considering Material Heterogeneity in Crack Modeling of Asphaltic Mixtures." Transportation Research Record: Journal of the Transportation Research Board, 1832(-1), 113-120.

Song, I., Little, D., Masad, E., Lytton, R., You, Z., Rowe, G., Roque, R., Kim, R., Huang, S., and Dongre, R. (Year). "Comprehensive evaluation of damage in asphalt mastics using X-ray CT, continuum mechanics, and micromechanics." Association of Asphalt Paving Technologist, White Bear Lake, MN 55110, United States, Long Beach, CA, United States, 885-920.

Song, S. H., Paulino, G. H., and Buttlar, W. G. (2006a). "A bilinear cohesive zone model tailored for fracture of asphalt concrete considering viscoelastic bulk material." Engineering Fracture Mechanics, 73(18), 2829-2848.

Song, S. H., Paulino, G. H., and Buttlar, W. G. (2006b). "Simulation of crack propagation in asphalt concrete using an intrinsic cohesive zone model." Journal of Engineering Mechanics, 132(11), 1215-1223.

Spring, D. W. (2011). "Cohesive Zone Modeling of Fracture of Sustainable and Functionally Graded Concrete," University of Illinois at Urbana-Champaign, Urbana, Illinois. 
Stolarska, M., Chopp, D. L., Moës, N., and Belytschko, T. (2001). "Modelling crack growth by level sets in the extended finite element method." International Journal for Numerical Methods in Engineering, 51(8), 943-960.

Sukumar, N., Chopp, D. L., Moës, N., and Belytschko, T. (2001). "Modeling holes and inclusions by level sets in the extended finite-element method." Computer Methods in Applied Mechanics and Engineering, 190(46-47), 6183-6200.

Sukumar, N., Huang, Z. Y., Pr関ost, J.-H., and Suo, Z. (2004). "Partition of unity enrichment for bimaterial interface cracks." International Journal for Numerical Methods in Engineering, 59(8), 1075-1102.

Sukumar, N., and Préevost, J. H. (2003). "Modeling quasi-static crack growth with the extended finite element method Part I: Computer implementation." International Journal of Solids and Structures, 40(26), 7513-7537.

Sullivan, E. J. (2006). Long-Term Cement Consumption Outlook, Portland Cement Association.

Sumanasooriya, M. S., Bentz, D. P., and Neithalath, N. (2010). "Planar Image-Based Reconstruction of Pervious Concrete Pore Structure and Permeability Prediction." ACI Materials Journal, 107(4), 413-421.

Sumanasooriya, M. S., and Neithalath, N. (2009). "Stereology- and Morphology-Based Pore Structure Descriptors of Enhanced Porosity (Pervious) Concretes." ACI Materials Journal, 106(5), 429-438.

Sun, Z., and Scherer, G. W. (2010a). "Effect of air voids on salt scaling and internal freezing." Cement and Concrete Research, 40(2), 260-270.

Sun, Z., and Scherer, G. W. (2010b). "Measurement and simulation of dendritic growth of ice in cement paste." Cement and Concrete Research, 40(9), 1393-1402.

Terzic, A. M., and Pavlovic, L. M. (2010). "Application of Results of Nondestructive Testing Methods in the Investigation of Microstructure of Refractory Concretes." Journal of Materials in Civil Engineering, 22(9), 853-857.

Ueda, T., Hasan, M., Nagai, K., Sato, Y., and Wang, L. (2009). "Mesoscale Simulation of Influence of Frost Damage on Mechanical Properties of Concrete." American Society of Civil Engineers, pp 244-252.

Valenza II, J., and Scherer, G. (2005). "Mechanisms of salt scaling." Materials and Structures, 38(4), 479-488.

Valenza II, J. J., and Scherer, G. W. (2007). "A review of salt scaling: II. Mechanisms." Cement and Concrete Research, 37(7), 1022-1034. 
van Mier, J. G. M. (2008). "Framework for a generalized four-stage fracture model of cement-based materials." Engineering Fracture Mechanics, 75(18), 5072-5086.

Ventura, G., Budyn, E., and Belytschko, T. (2003). "Vector level sets for description of propagating cracks in finite elements." International Journal for Numerical Methods in Engineering, 58(10), 1571-1592.

Voigt, W. (1889). "Ueber die Beziehung zwischen den beiden Elasticitätsconstanten isotroper Körper." Annalen der Physik, 274(12), 573-587.

Williams, M. (1957). "On the stress distribution at the base of a stationary crack." Journal of Applied Mechanics, 24, 109-114.

Wriggers, P., and Moftah, S. O. (2006). "Mesoscale models for concrete: Homogenisation and damage behaviour." Finite Elements in Analysis and Design, 42(7), 623-636.

$\mathrm{Xu}, \mathrm{X}$. P., and Needleman, A. (1994). "Numerical simulations of fast crack growth in brittle solids." Journal of the Mechanics and Physics of Solids, 42(9), 1397-1434.

You, Z., Adhikari, S., and Emin Kutay, M. (2009). "Dynamic modulus simulation of the asphalt concrete using the X-ray computed tomography images." Materials and Structures, 42(5), 617-630.

You, Z., and Buttlar, W. G. (2005). "Application of Discrete Element Modeling Techniques to Predict the Complex Modulus of Asphalt-Aggregate Hollow Cylinders Subjected to Internal Pressure." Journal of the Transportation Research Board, National Research Council, 1929, 218-226.

You, Z., and Buttlar, W. G. (2006). "Micromechanical Modeling Approach to Predict Compressive Dynamic Moduli of Asphalt Mixture Using the Distinct Element Method." Journal of the Transportation Research Board, National Research Council, Washington, D.C., 1970, 73-83.

Zhou, Z.-Y., and Mihashi, H. (2008). "Micromechanics Model to Describe Strain Behavior of Concrete in Freezing Process." Journal of Materials in Civil Engineering, 20, 46-53. 


\section{APPENDIX}




\section{Appendix A: Developed MATLAB scheme to generate ABAQUS input file for implementing bilinear cohesive zone model (CZM)}

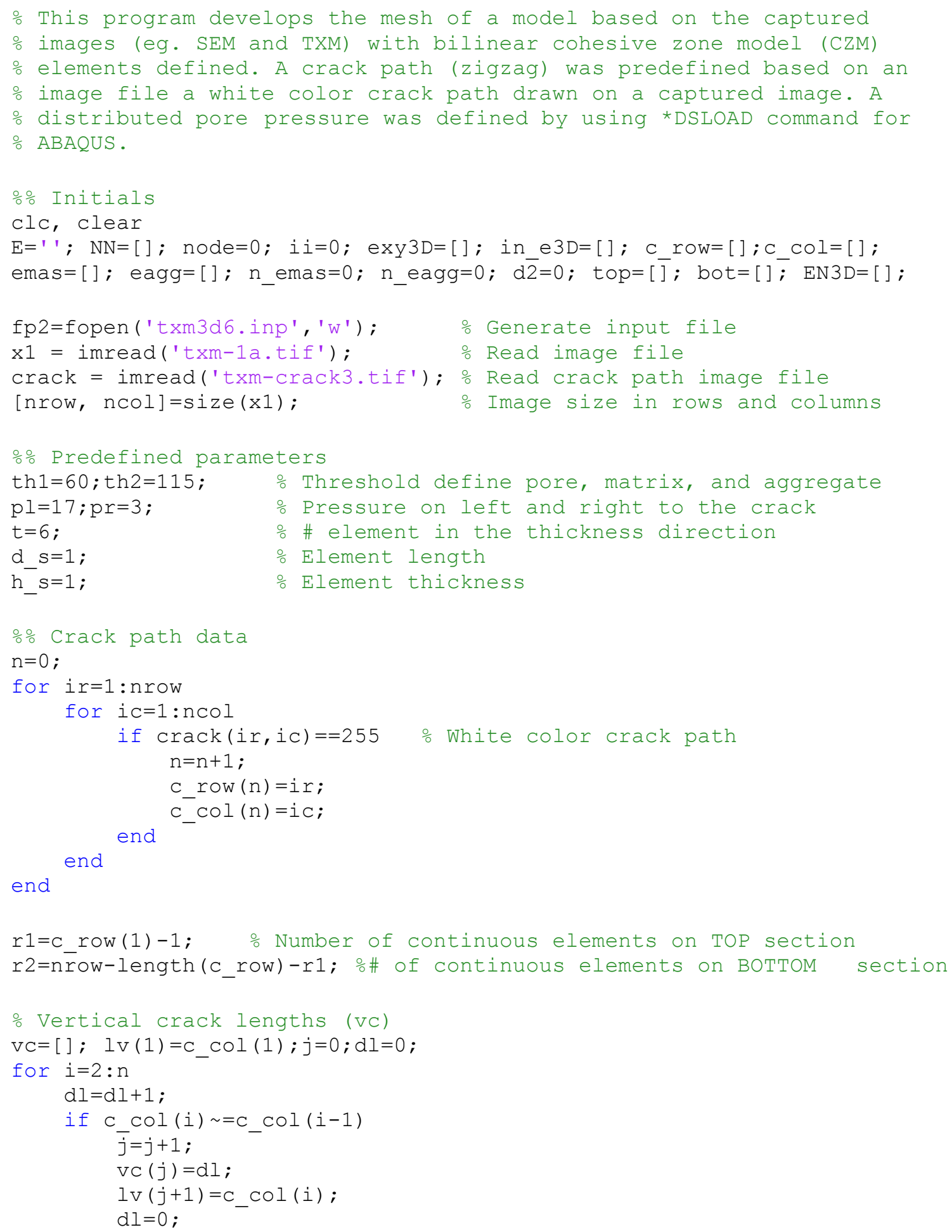




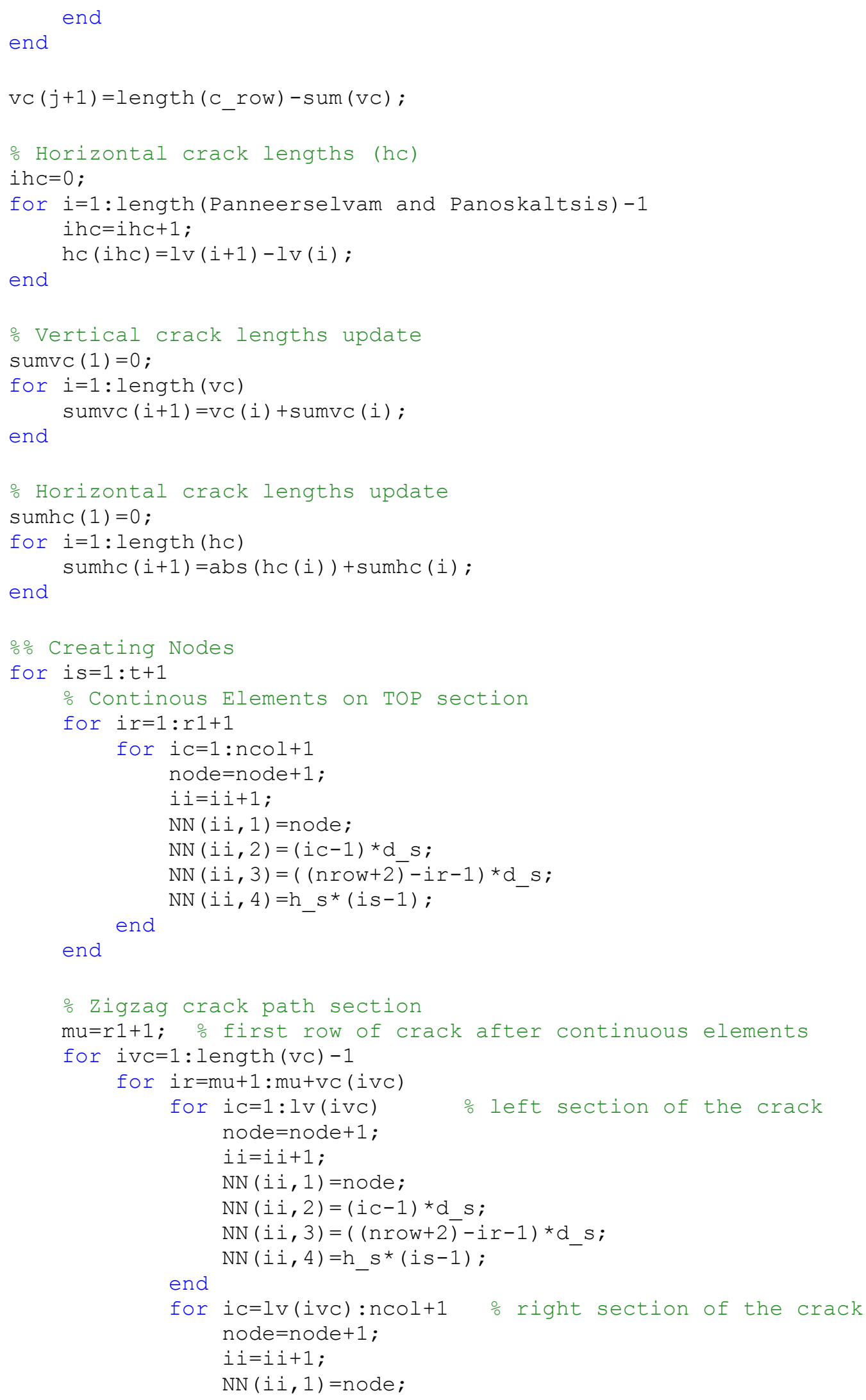




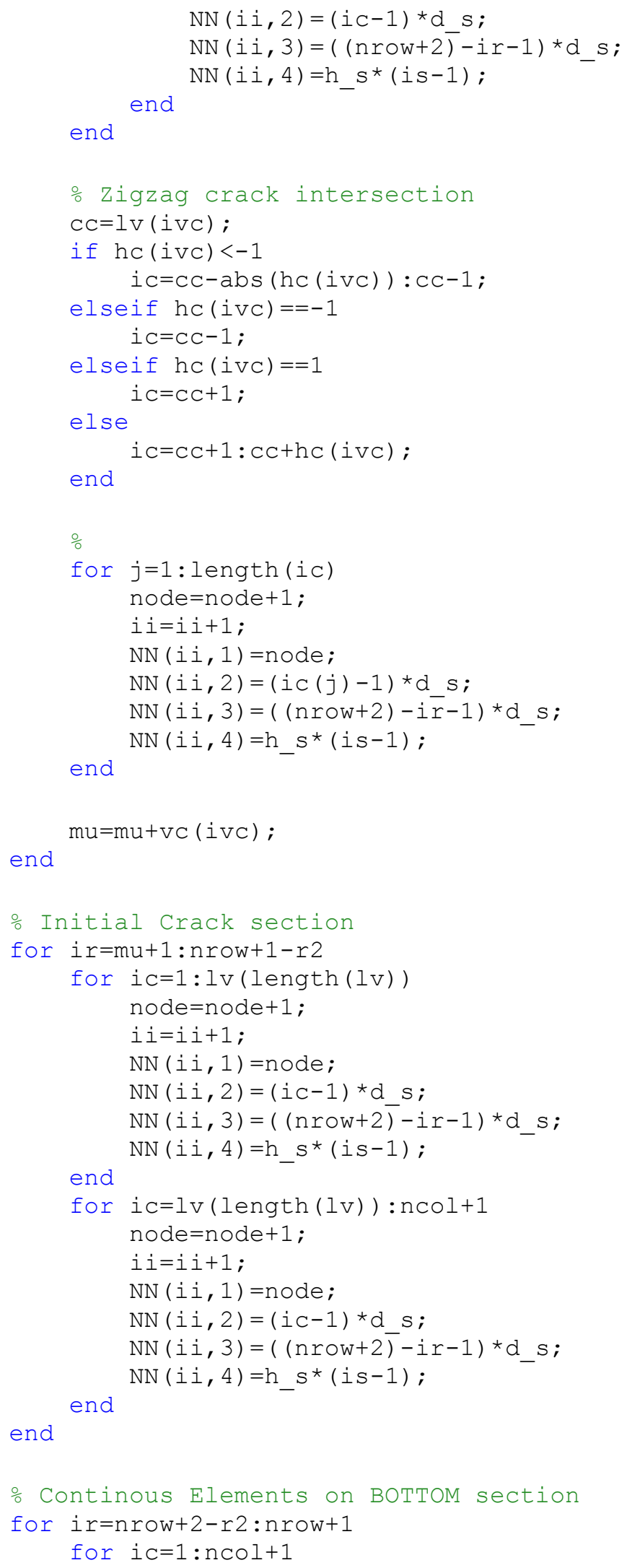




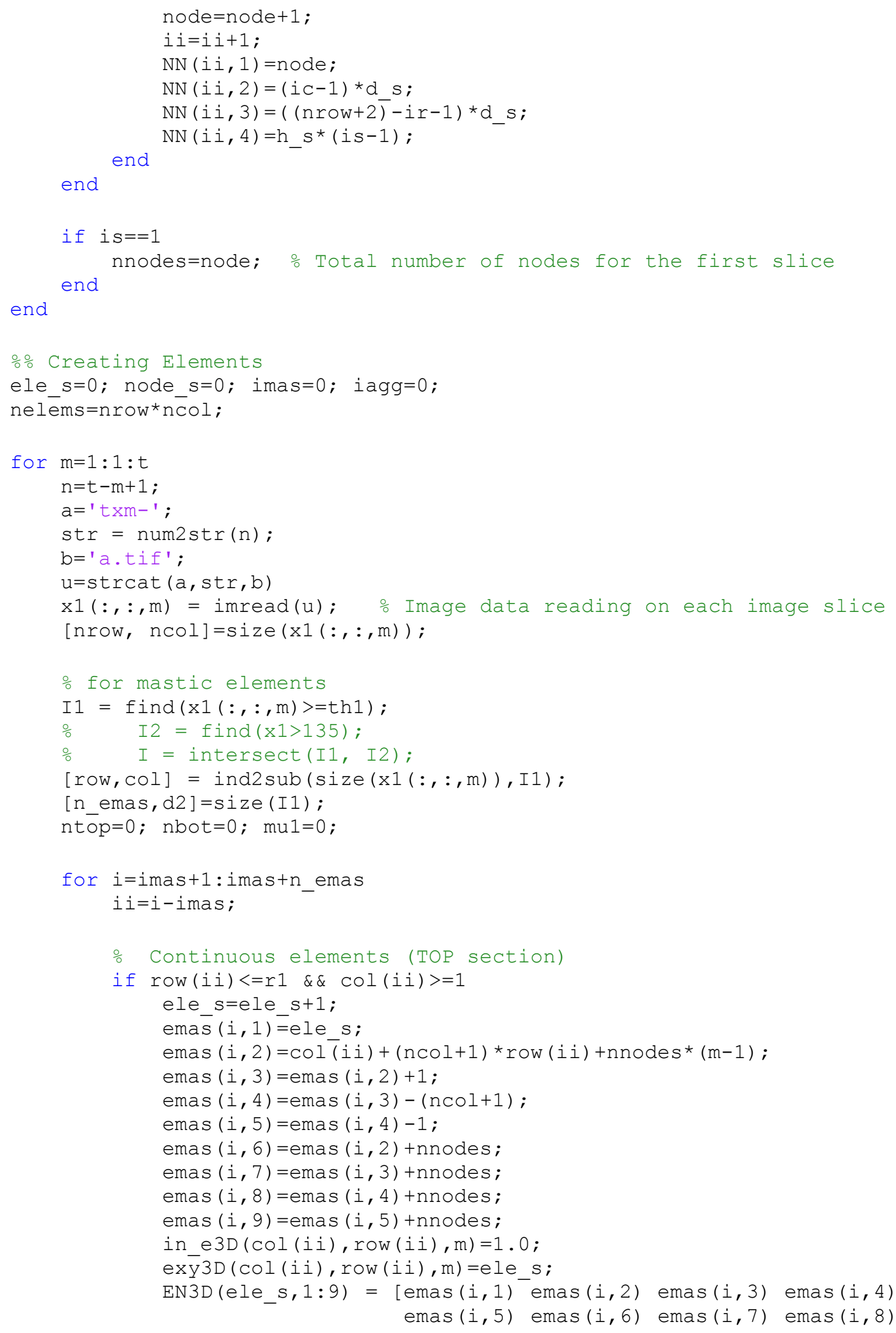




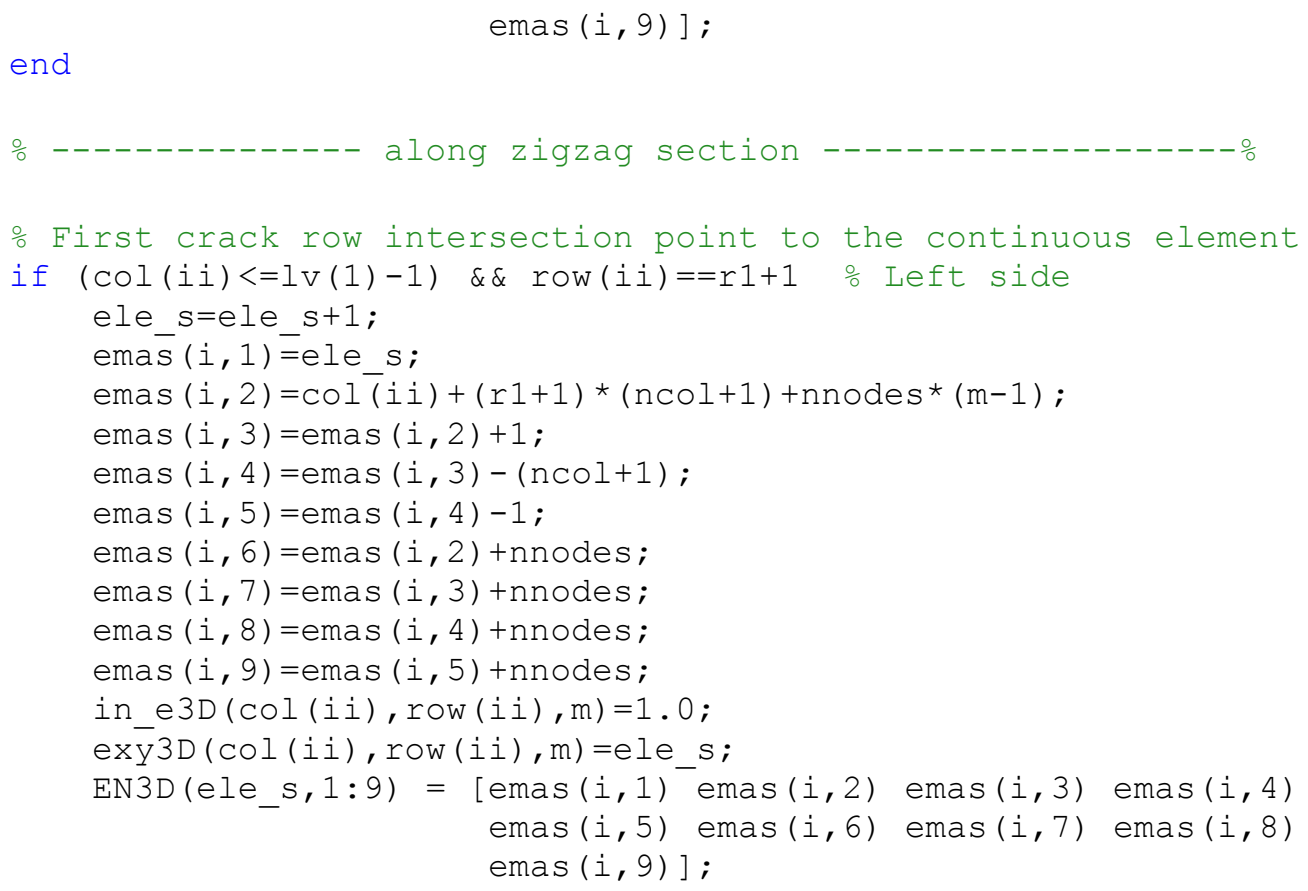

end

if $(\operatorname{col}(i i)>=\operatorname{lv}(1)) \quad \& \&$ row $(i i)==r 1+1$ ight side

ele_s=ele_s+1;

$\operatorname{emas}(i, 1)=e l e \_s$;

$\operatorname{emas}(i, 2)=\operatorname{col}(i \mathrm{i})+(\mathrm{r} 1+1) *(\operatorname{ncol}+1)+1+\operatorname{nnodes} *(m-1)$;

$\operatorname{emas}(i, 3)=\operatorname{emas}(i, 2)+1$;

$\operatorname{emas}(i, 4)=\operatorname{emas}(i, 3)-(\operatorname{ncol}+2)$;

$\operatorname{emas}(i, 5)=\operatorname{emas}(i, 4)-1$;

$\operatorname{emas}(i, 6)=\operatorname{emas}(i, 2)+$ nnodes ;

$\operatorname{emas}(i, 7)=\operatorname{emas}(i, 3)+$ nnodes ;

emas $(i, 8)=\operatorname{emas}(i, 4)+$ nnodes ;

emas $(i, 9)=\operatorname{emas}(i, 5)+$ nnodes

in_e3D( $\operatorname{col}(\mathrm{i} i), \operatorname{row}(\mathrm{i} i), \mathrm{m})=1.0$;

exȳ3D (col (ii), row (ii), m) =ele_s;

$\operatorname{EN3D}($ ele_s, $1: 9)=[\operatorname{emas}(i, 1) \operatorname{emas}(i, 2) \operatorname{emas}(i, 3) \operatorname{emas}(i, 4)$

emas $(i, 5)$ emas $(i, 6)$ emas $(i, 7)$ emas $(i, 8)$ $\operatorname{emas}(i, 9)]$;

end

o Zigzag crack rows after intersection point

for $i \mathrm{VC}=1:$ length $(\mathrm{VC})$

$\mathrm{mu}=\mathrm{r} 1+1$;

if $(\operatorname{col}(i i)<=\operatorname{lv}(i v c)-1) \quad \& \&$ row $(i i)>=\operatorname{mu}+1+\operatorname{sumvc}(i v c) \quad \ldots$

$\& \&$ row $(i i)<=m u+\operatorname{sumvc}(i v c+1)-1$ 낭 side

ele s=ele $s+1$;

$\operatorname{ema} \bar{s}(i, 1)=$ ele $s$;

$\operatorname{emas}(i, 2)=\operatorname{col}(i \mathrm{i})+(\operatorname{rl}+1) *(\operatorname{ncol}+1)+(\operatorname{row}(i \mathrm{i})-\mathrm{mu}) *(\mathrm{ncol}+2)$ $+\operatorname{sumhc}(i v c)+$ nnodes* $(m-1)$;

$\operatorname{emas}(i, 3)=\operatorname{emas}(i, 2)+1$;

$\operatorname{emas}(i, 4)=\operatorname{emas}(i, 3)-(\operatorname{ncol}+2)$; 


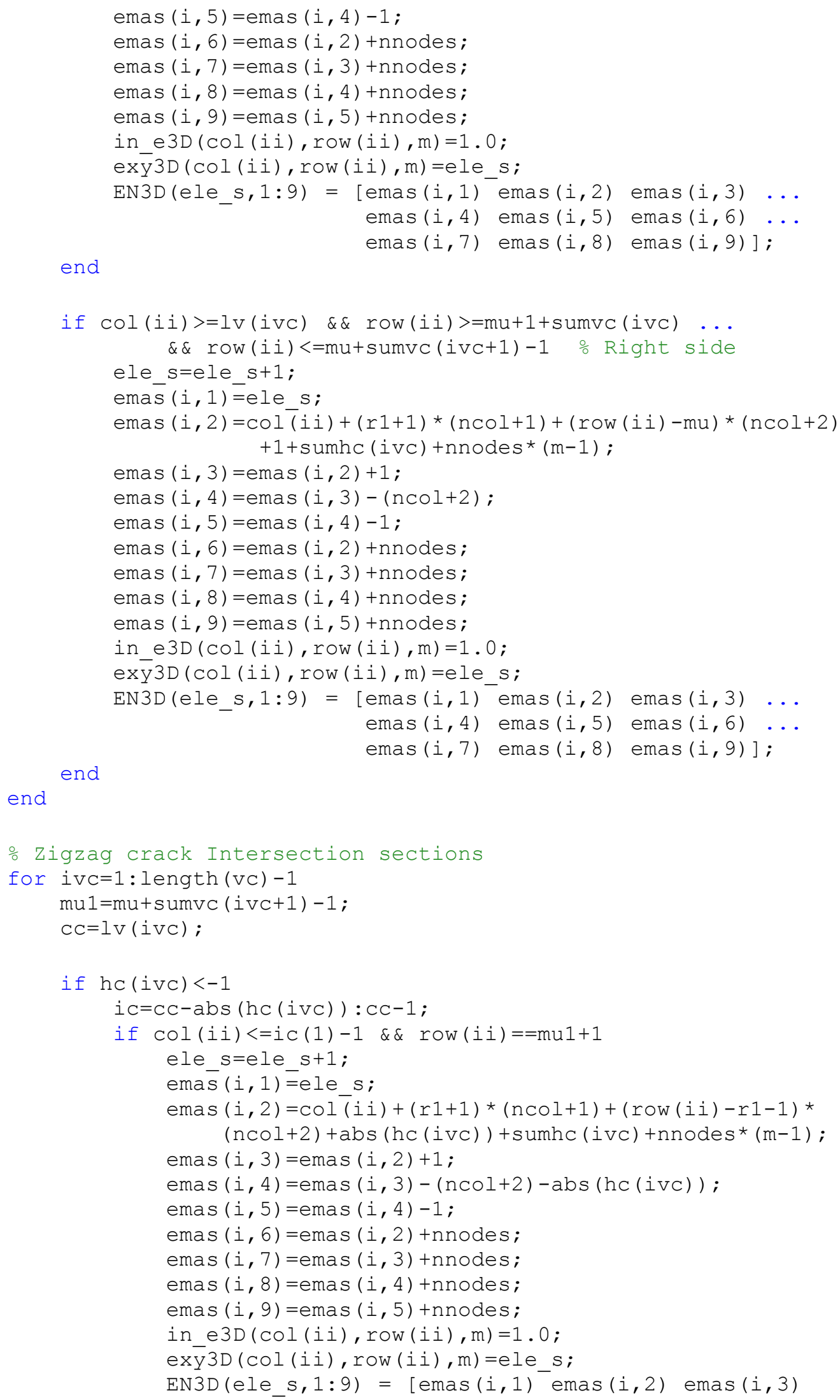

end

if $\operatorname{col}($ ii) $>=l v(i v C) \quad \& \&$ row $(i i)>=m u+1+\operatorname{sumvc}(i v c) \ldots$ $\& \& \operatorname{row}(i \mathrm{i})<=\mathrm{mu}+\operatorname{sumvc}(i \mathrm{vc}+1)-1$ 을 $\operatorname{Right}$ side 
end

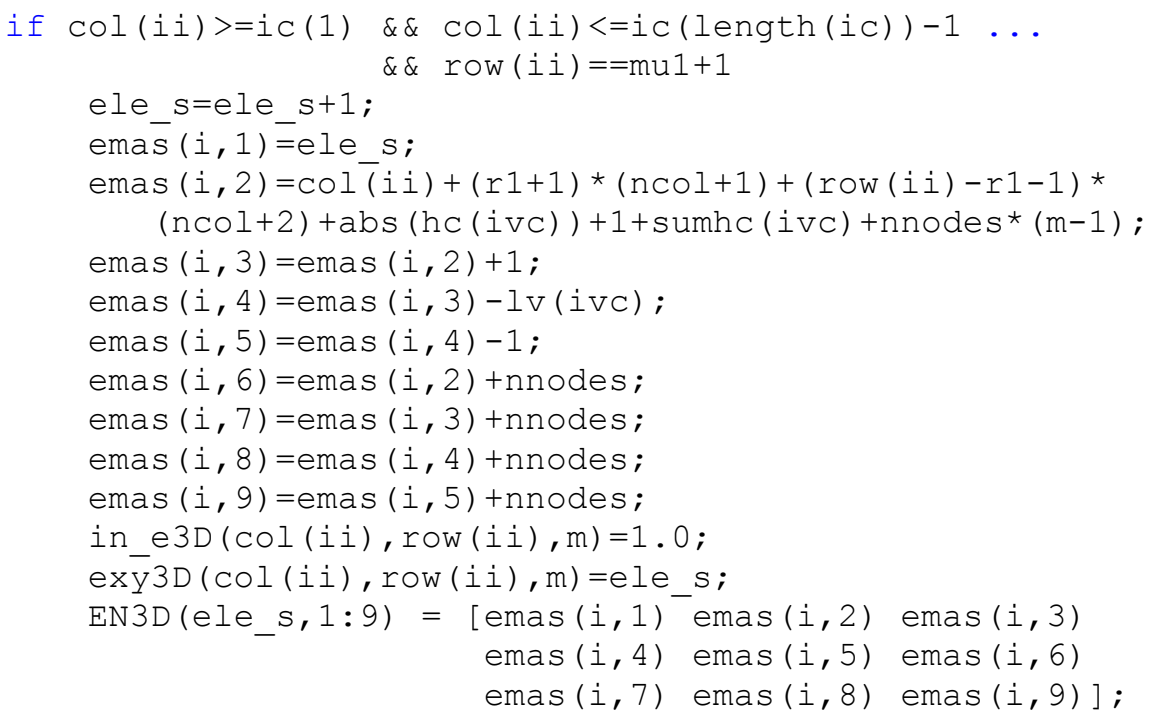

end

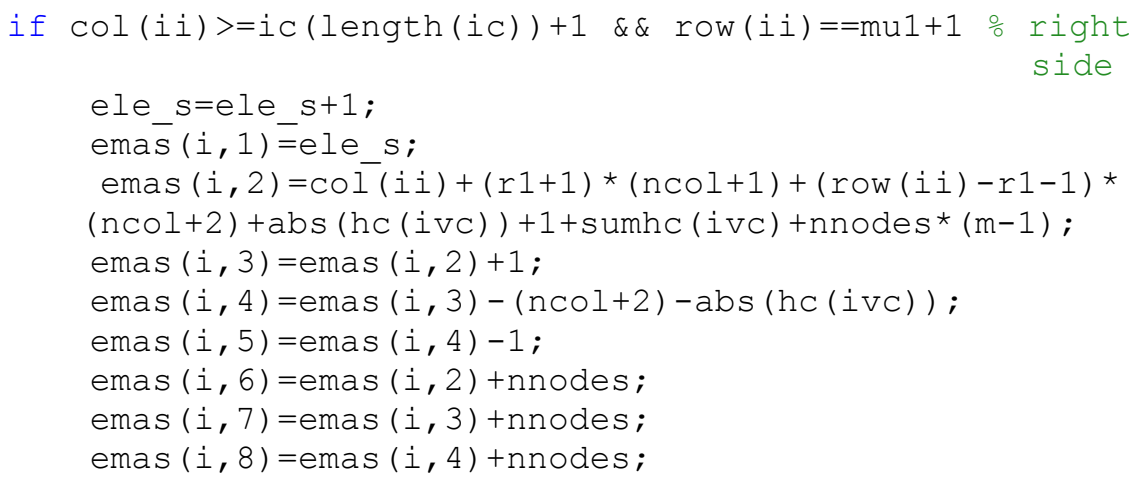




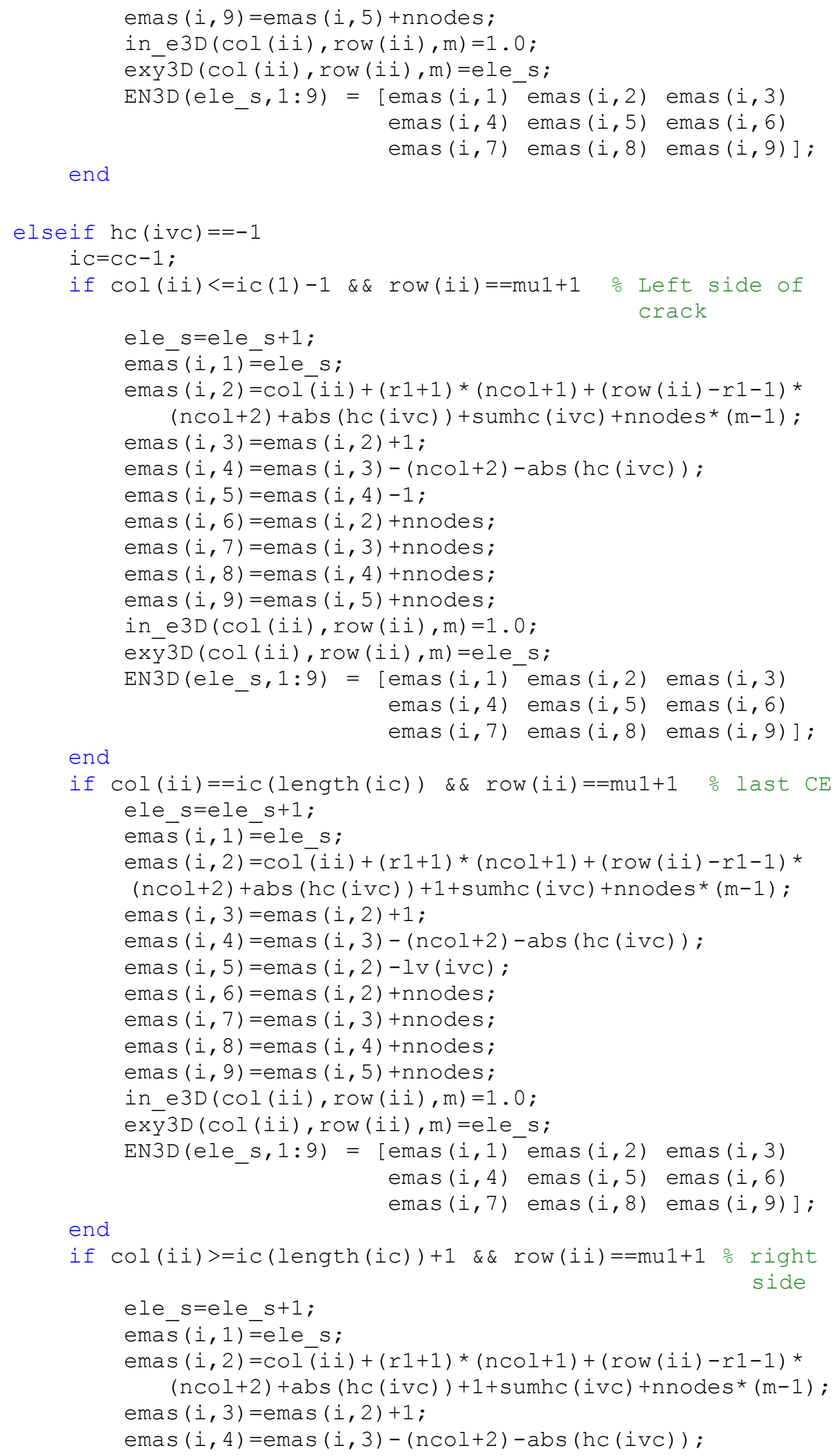




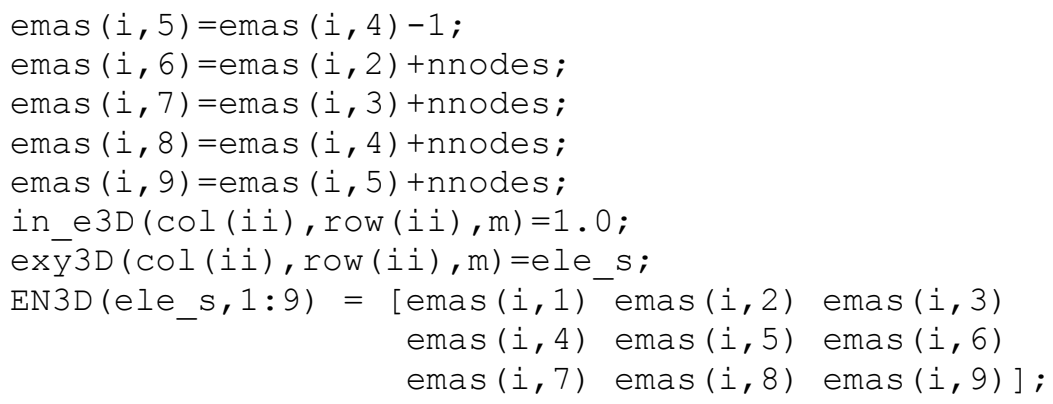

end

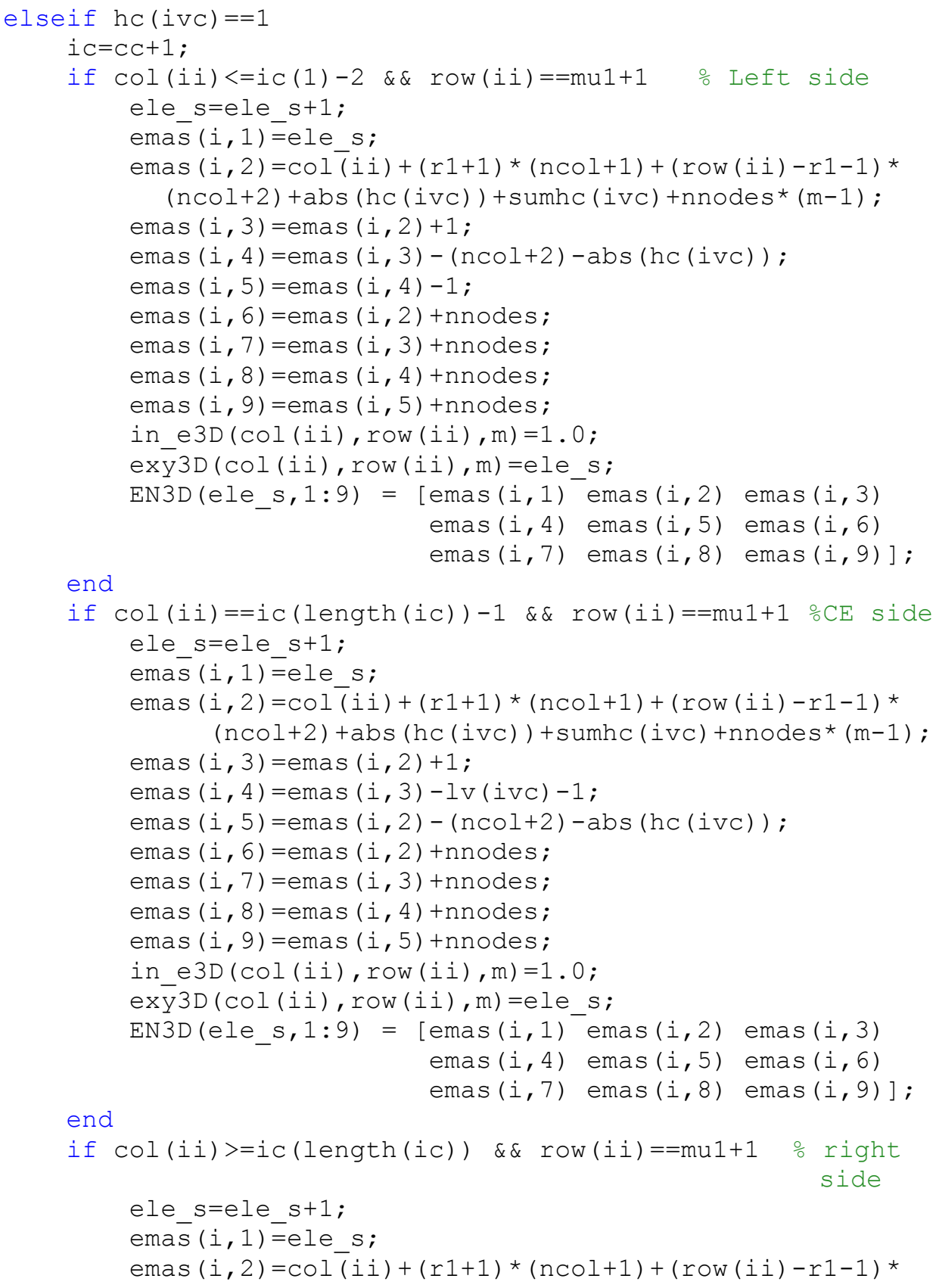


$(\mathrm{ncol}+2)+\mathrm{abs}(\mathrm{hc}(\mathrm{iVc}))+1+\operatorname{sumhc}($ ivc $)+$ nnodes $*(m-1)$; $\operatorname{emas}(i, 3)=\operatorname{emas}(i, 2)+1$;

$\operatorname{emas}(i, 4)=\operatorname{emas}(i, 3)-(\operatorname{ncol}+2)-\operatorname{abs}(\operatorname{hc}(i \mathrm{Vc}))$;

$\operatorname{emas}(i, 5)=\operatorname{emas}(i, 4)-1$;

emas $(i, 6)=\operatorname{emas}(i, 2)+$ nnodes ;

emas $(i, 7)=\operatorname{emas}(i, 3)+$ nnodes ;

$\operatorname{emas}(i, 8)=\operatorname{emas}(i, 4)+$ nnodes ;

emas $(i, 9)=\operatorname{emas}(i, 5)+$ nnodes ;

in_e3D( $\operatorname{col}(i \mathrm{i}), \operatorname{row}(\mathrm{i} i), \mathrm{m})=1.0$;

exȳ3D (col (ii), row (ii), m) =ele_s;

EN3D $\left(e l e \_s, 1: 9\right)=[\operatorname{emas}(i, 1) \operatorname{emas}(i, 2) \operatorname{emas}(i, 3)$

$\operatorname{emas}(i, 4) \operatorname{emas}(i, 5) \operatorname{emas}(i, 6)$

emas $(i, 7)$ emas $(i, 8)$ emas $(i, 9)]$;

end

else

ic $=\mathrm{CC}-1: \mathrm{cC}+\mathrm{hC}(\mathrm{iVC})$;

if $\operatorname{col}(i i)<=i c(1) \quad \& \& \operatorname{row}(i i)==m u 1+1$ Left side

ele_s=ele_s+1;

$\operatorname{emas}(i, 1)=e l e \_s$;

$\operatorname{emas}(i, 2)=\operatorname{col}(i i)+(r 1+1) *(\operatorname{ncol}+1)+(\operatorname{row}(i i)-r 1-1)$ *

$(\mathrm{ncol}+2)+\mathrm{abs}(\mathrm{hc}(\mathrm{ivc}))+\operatorname{sumhc}(i \mathrm{Vc})+$ nnodes* $(m-1)$;

$\operatorname{emas}(i, 3)=\operatorname{emas}(i, 2)+1$;

$\operatorname{emas}(i, 4)=\operatorname{emas}(i, 3)-(\operatorname{ncol}+2)-\operatorname{abs}(\operatorname{hc}(i \vee c))$;

$\operatorname{emas}(i, 5)=\operatorname{emas}(i, 4)-1$;

$\operatorname{emas}(i, 6)=\operatorname{emas}(i, 2)+$ nnodes $;$

$\operatorname{emas}(i, 7)=\operatorname{emas}(i, 3)+$ nnodes ;

emas $(i, 8)=\operatorname{emas}(i, 4)+$ nnodes

emas $(i, 9)=\operatorname{emas}(i, 5)+$ nnodes ;

in e3D ( col (ii), row (ii), m) =1.0;

exȳ3D (col (ii), row (ii), m) =ele_s;

$\operatorname{EN3D}($ ele_s, $1: 9)=[\operatorname{emas}(i, 1) \operatorname{emas}(i, 2) \operatorname{emas}(i, 3)$

$\operatorname{emas}(i, 4) \operatorname{emas}(i, 5) \operatorname{emas}(i, 6)$

emas $(i, 7)$ emas $(i, 8)$ emas $(i, 9)]$;

end

if $\operatorname{col}(i \mathrm{i})==\mathrm{ic}(1)+1 \quad \& \&$ row $(\mathrm{ii})==\mathrm{mu} 1+1 \quad \circ \mathrm{CE}$ side

ele_s=ele_s+1;

$\operatorname{emas}(i, 1)=e l e \_s$;

$\operatorname{emas}(i, 2)=\operatorname{col}(i i)+(r 1+1) *(\operatorname{ncol}+1)+(\operatorname{row}(i i)-r 1-1)$ * $(\mathrm{ncol}+2)+\mathrm{abs}(\mathrm{hc}(\mathrm{ivc}))+\operatorname{sumh}(\mathbf{i v c})+$ nnodes* $(m-1)$;

$\operatorname{emas}(i, 3)=\operatorname{emas}(i, 2)+1$;

$\operatorname{emas}(i, 4)=\operatorname{emas}(i, 3)-\operatorname{lv}(i \mathrm{Vc})-\mathrm{hc}(i \mathrm{Vc})$;

$\operatorname{emas}(i, 5)=\operatorname{emas}(i, 2)-(\operatorname{ncol}+2)-\operatorname{abs}(\operatorname{hc}(i \vee c))$;

$\operatorname{emas}(i, 6)=\operatorname{emas}(i, 2)+$ nnodes $;$

$\operatorname{emas}(i, 7)=\operatorname{emas}(i, 3)+$ nnodes ;

emas $(i, 8)=\operatorname{emas}(i, 4)+$ nnodes;

emas $(i, 9)=\operatorname{emas}(i, 5)+$ nnodes

in_e3D(col (ii), row $(i i), m)=1.0$;

exȳ3D (col (ii), row (ii), m) =ele_s;

$\operatorname{EN3D}\left(e l e \_s, 1: 9\right)=[\operatorname{emas}(i, 1) \operatorname{emas}(i, 2) \operatorname{emas}(i, 3)$

emas $(i, 4)$ emas $(i, 5)$ emas $(i, 6)$

$\operatorname{emas}(i, 7)$ emas $(i, 8)$ emas $(i, 9)]$;

end

if $\operatorname{col}($ ii) $>=$ ic $(1)+2 \& \& \operatorname{col}(i i)<=i c(l e n g t h(i c)) \ldots$

$\& \& \operatorname{row}(i)==\operatorname{mul} 1 \quad \div \mathrm{CE}$ side 


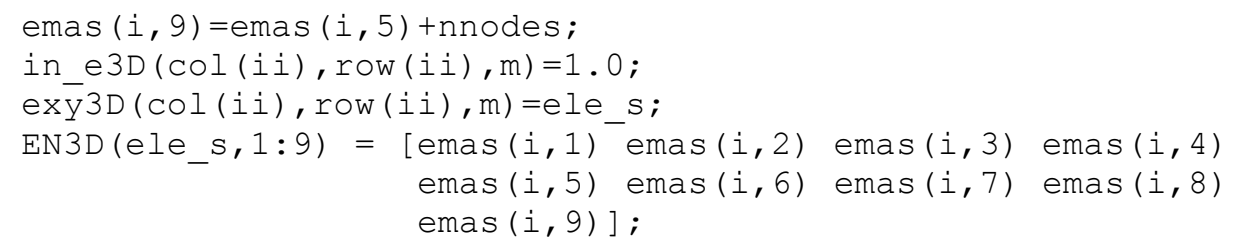

end 


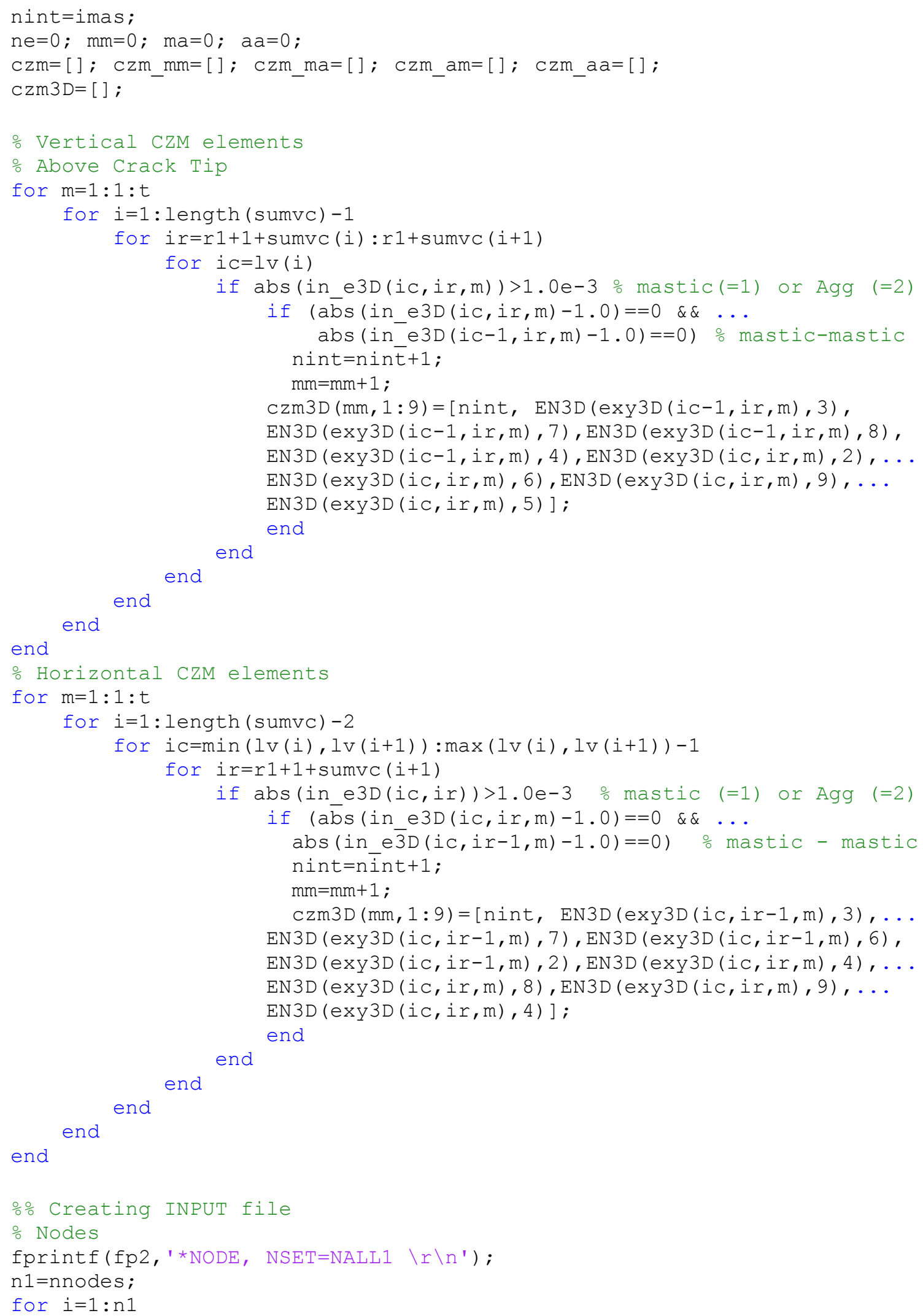




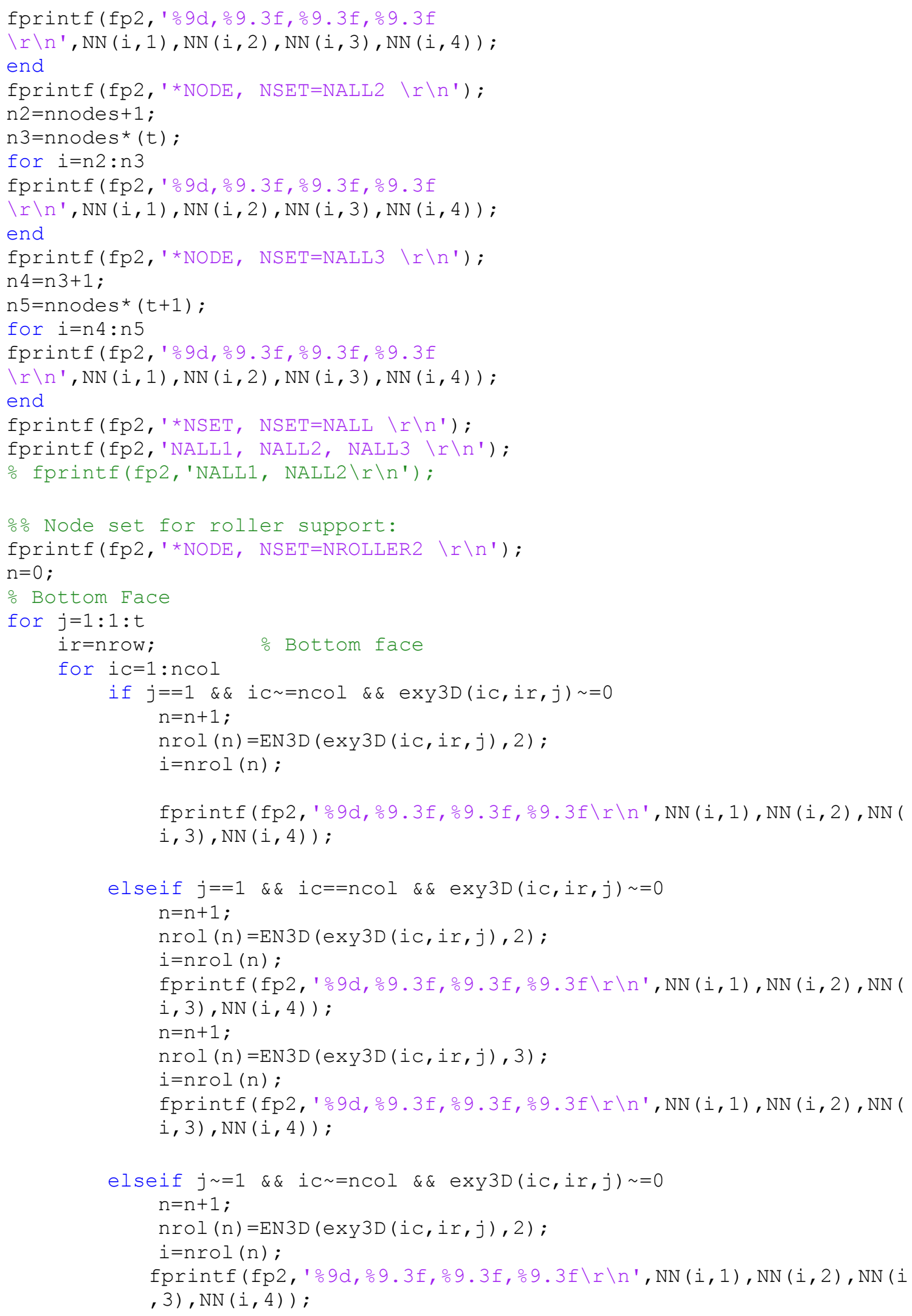




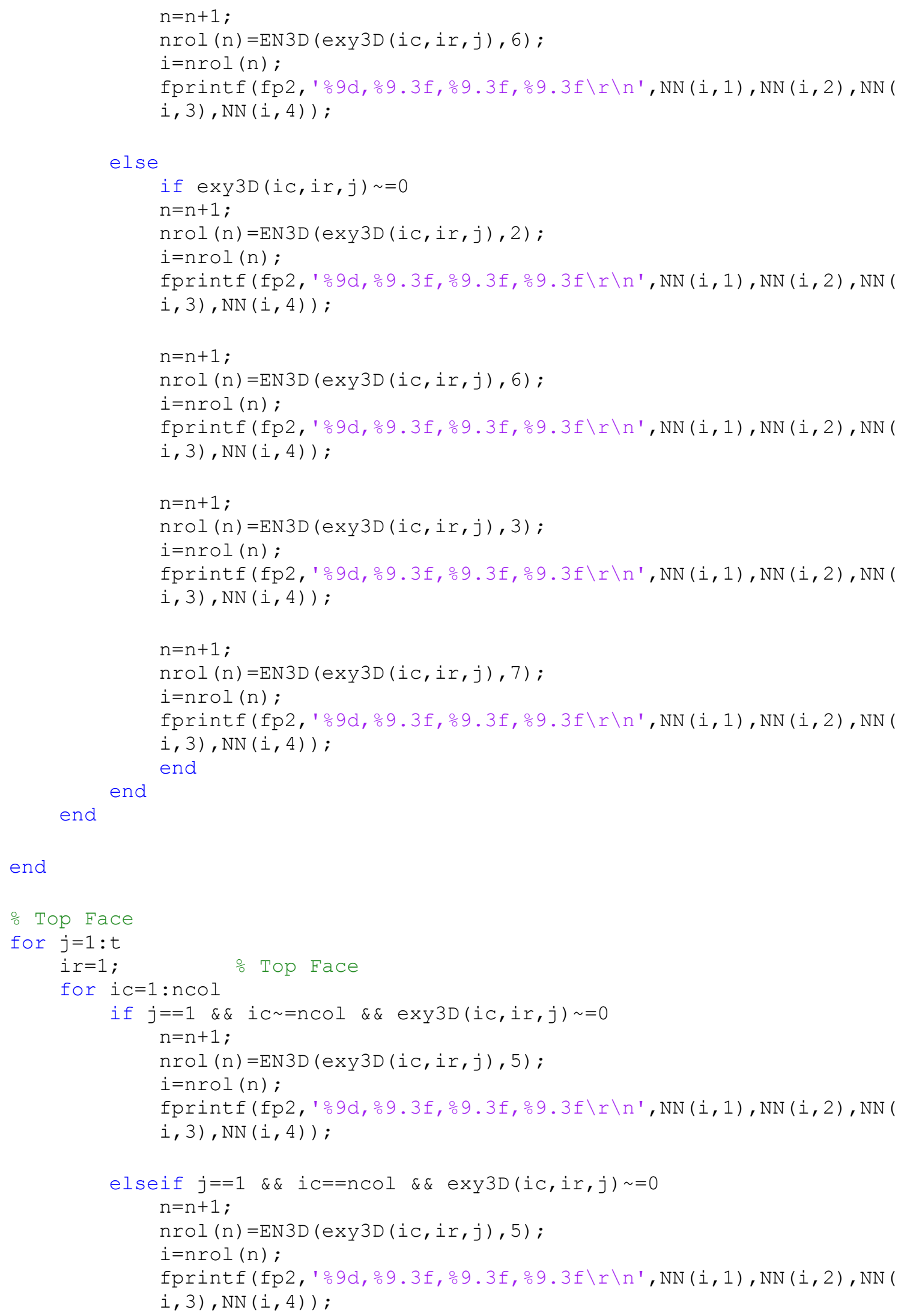




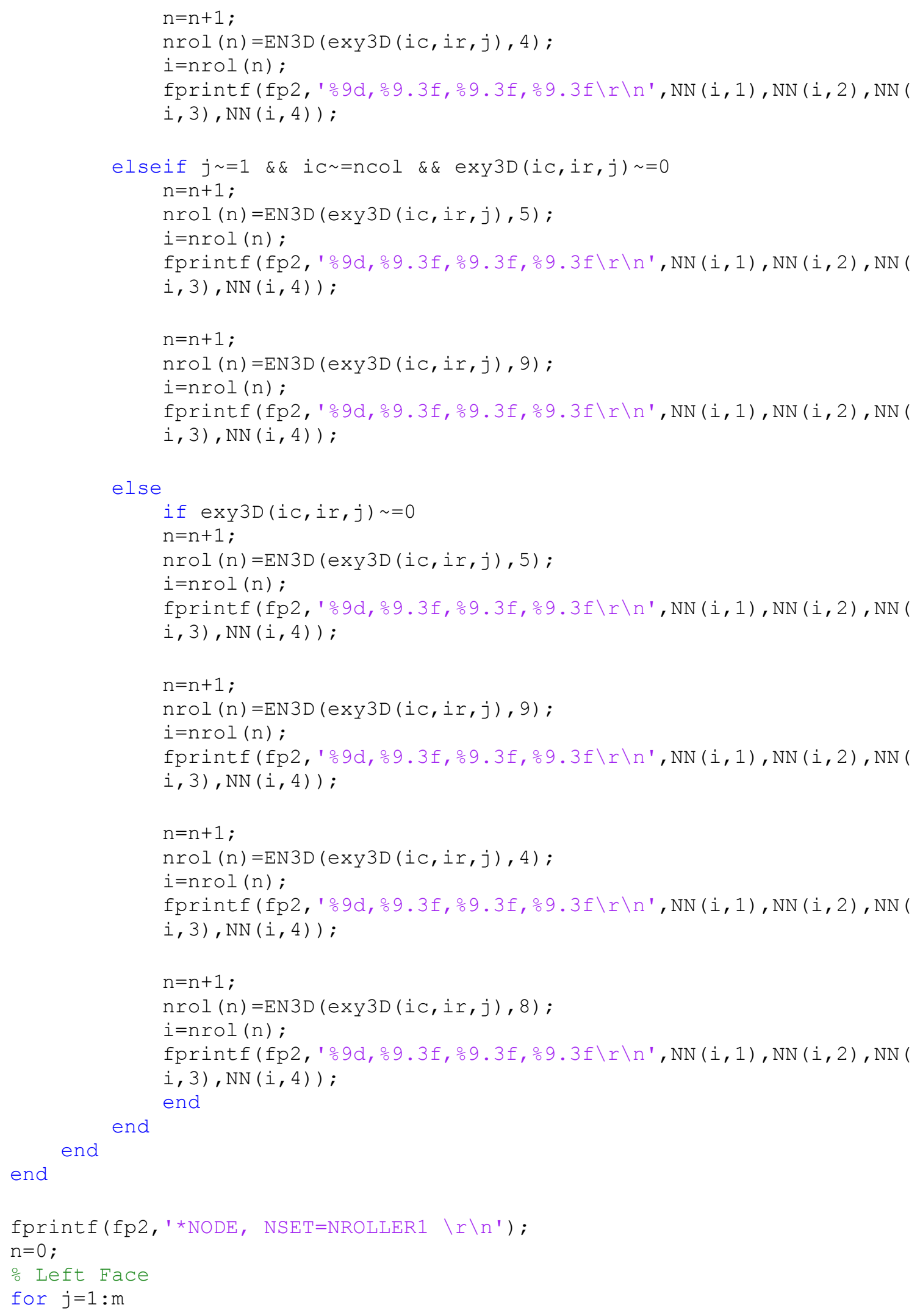




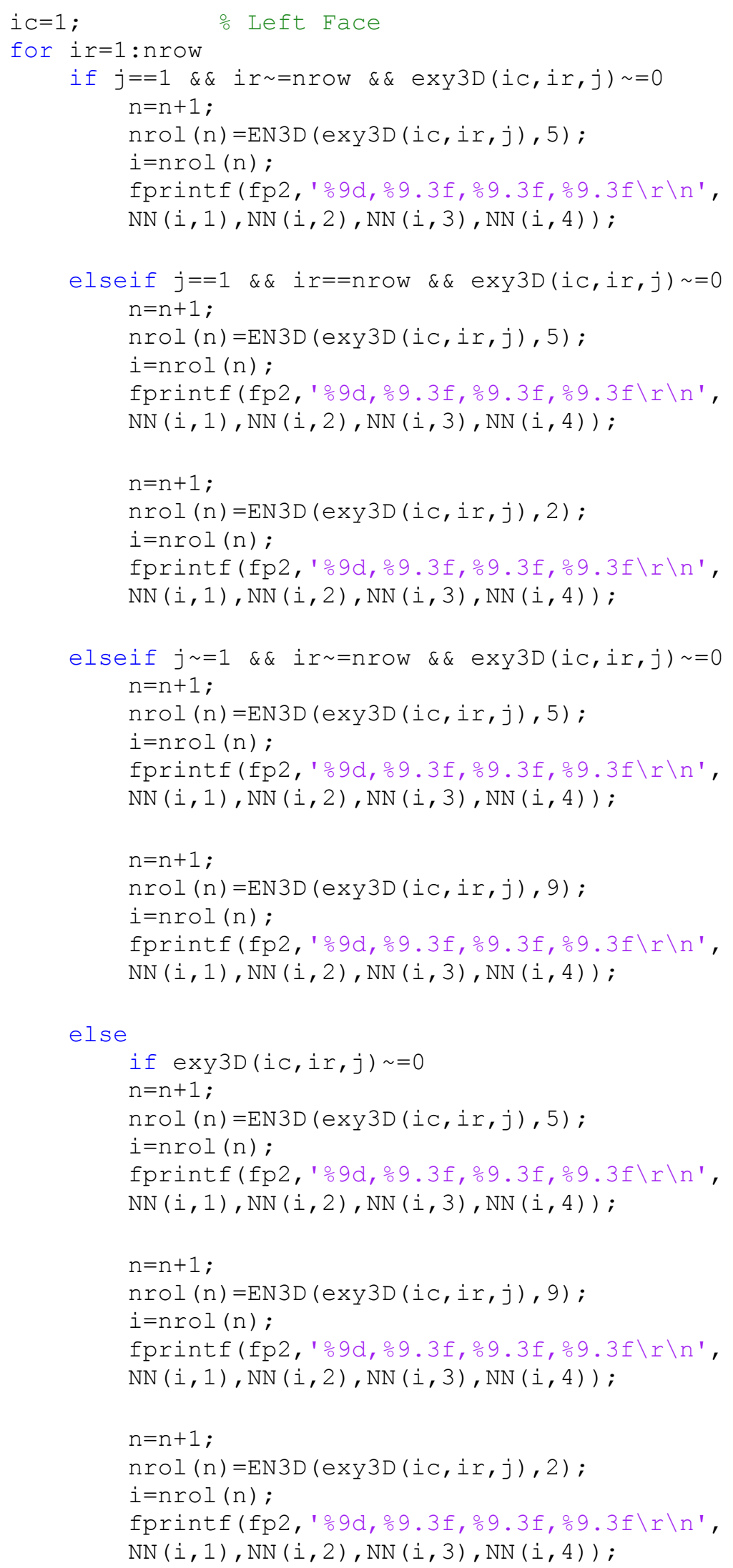




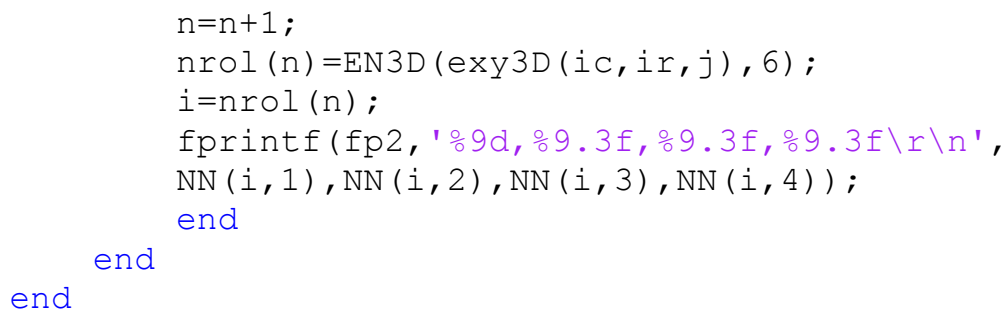

end

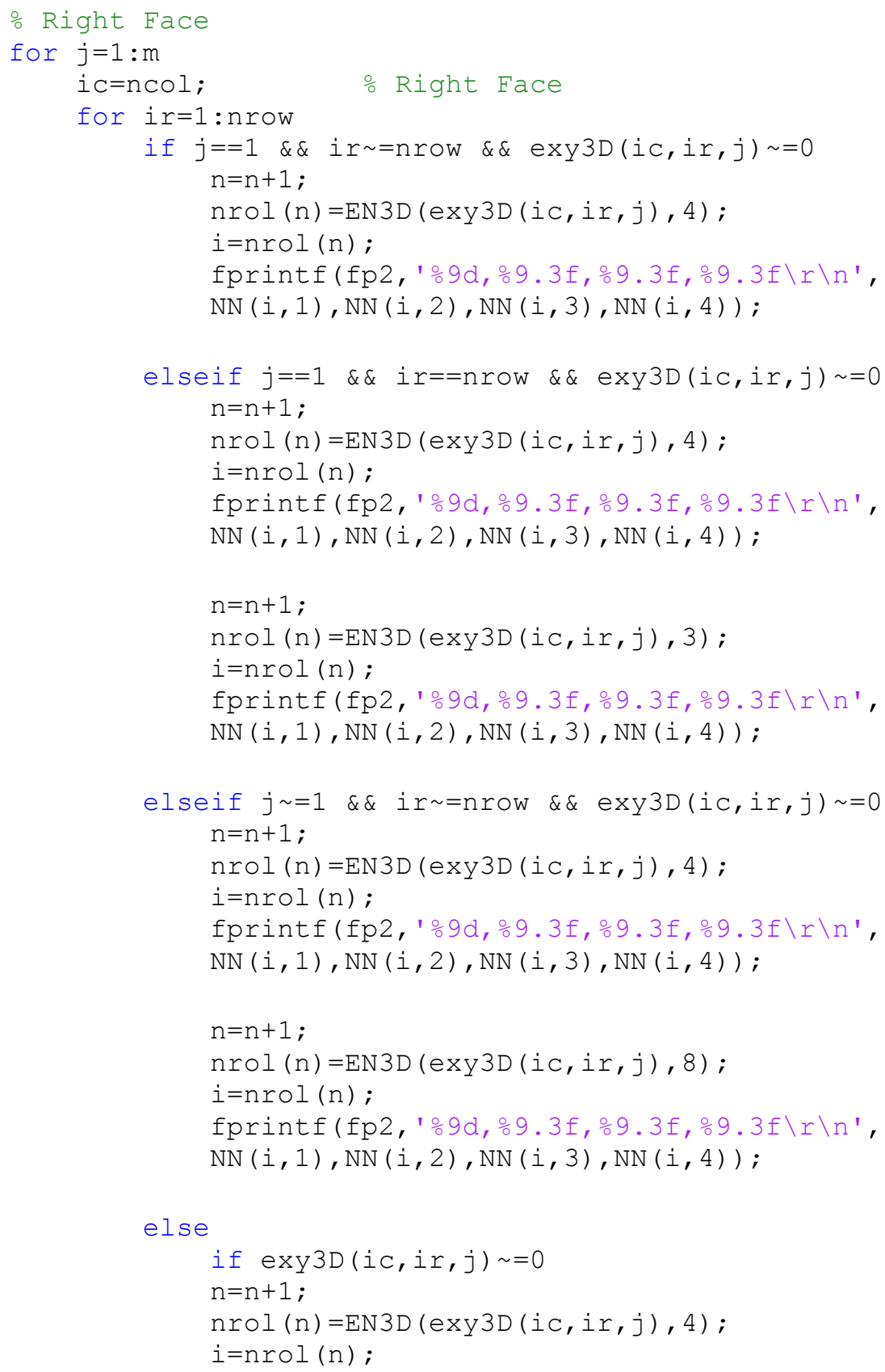




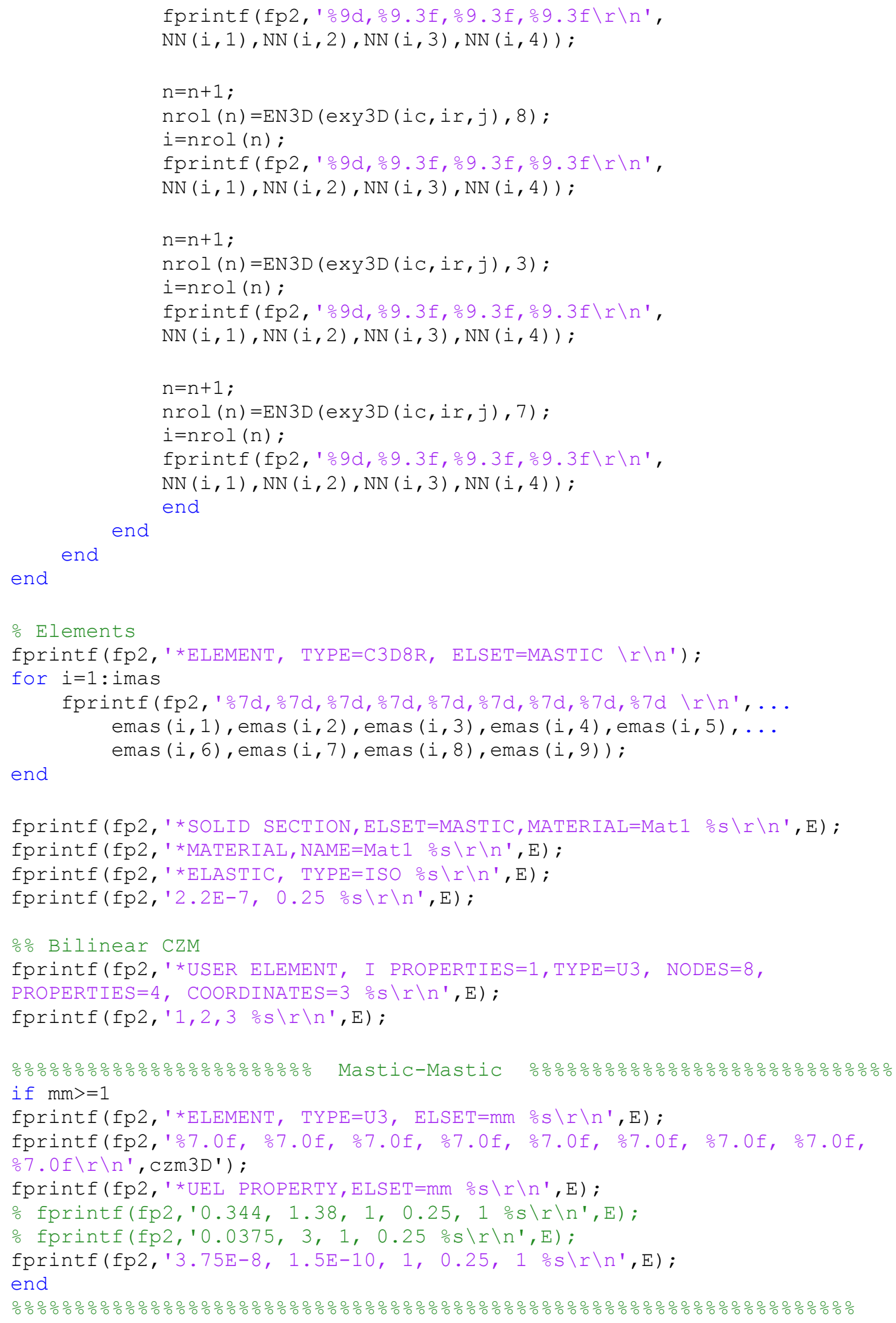




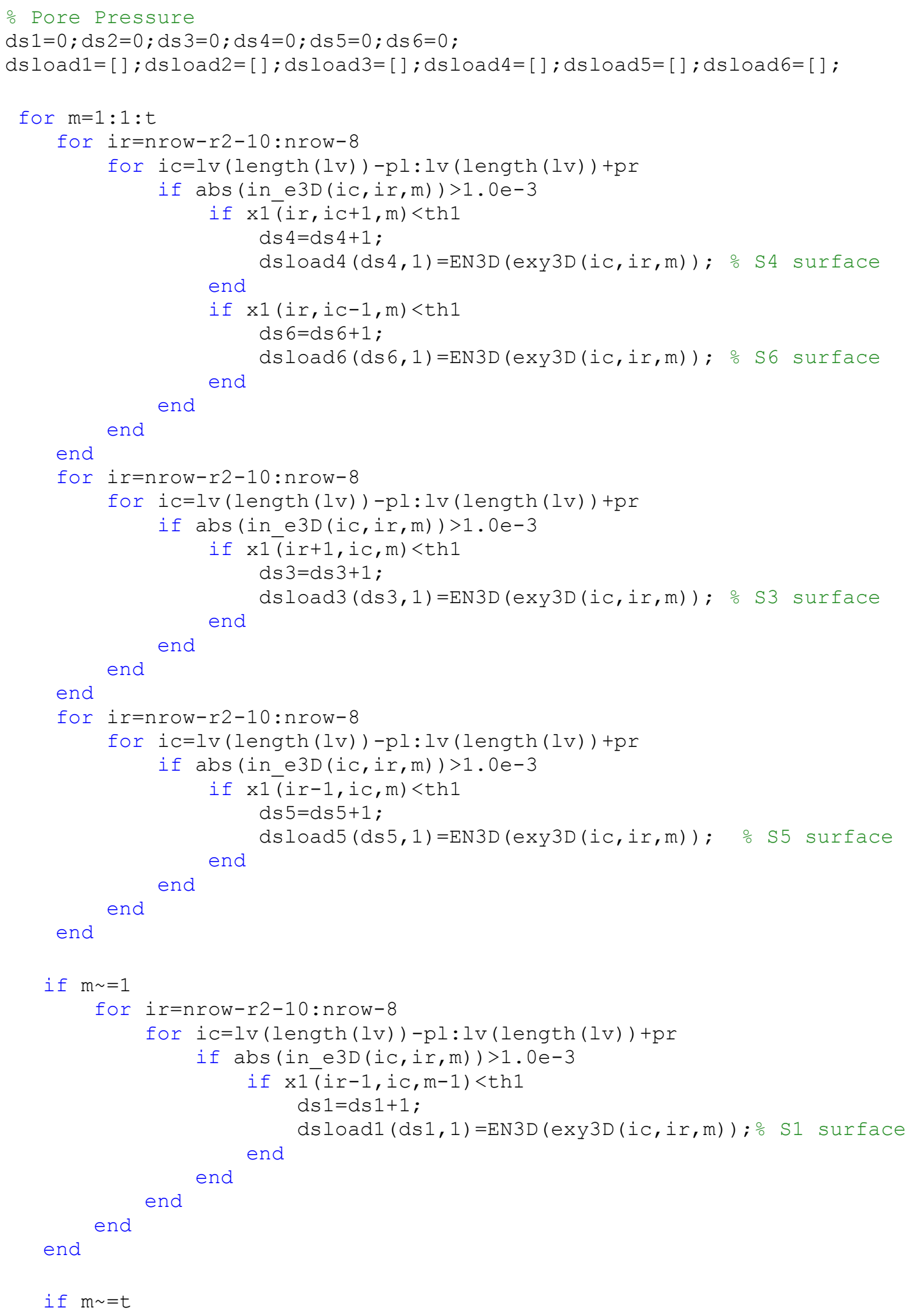




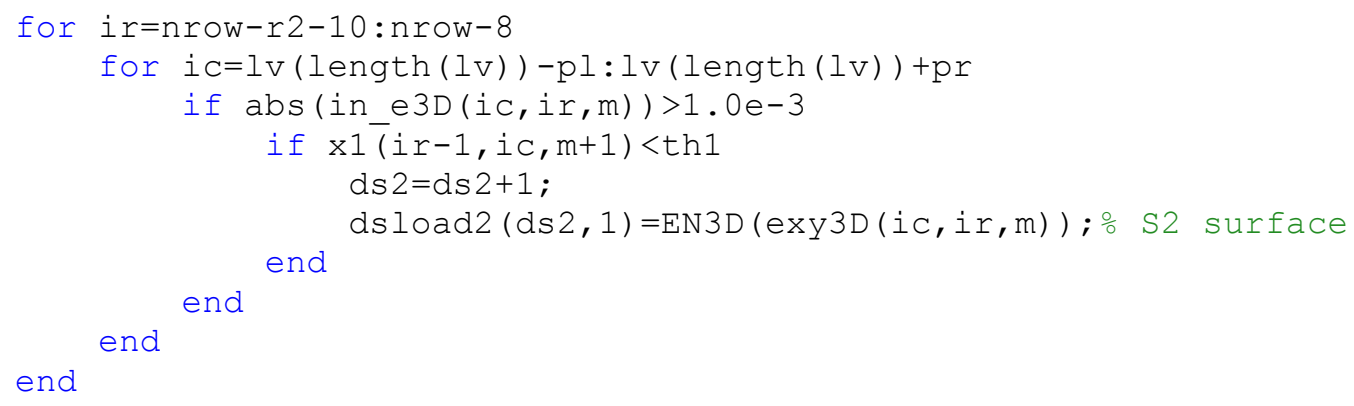




\section{Appendix B: Bilinear CZM user element subroutine (ABAQUS) for simulating fracture behavior in 3-D microstructure models}

c This 3-D bilinear CZM program was originally developed by Dr. Seong Hyeok Song (UIUC) and the program has been altered to allow this subroutine to be executed with microstructure-based computational model with ABAQUS.

C USER DEFINED ELEMENT

SUBROUTINE UEL(RHS, AMATRX, SVARS, ENERGY, NDOFEL, NRHS, NSVARS, + PROPS, NPROPS, COORDS, MCRD, NNODE, U, DU, V, A, jtype, TIME, DTIME,

+ KSTEP, KINC, jelem, PARAMS, NDLOAD, JDLTYP, ADLMAG, PREDEF, + NPREDF, LFLAGS, MLVARX, DDLMAG, MDLOAD, PNEWDT, JPROPS, NJPROP,

+ PERIOD)

INCLUDE 'ABA_PARAM.INC'

DIMENSION RHS(MLVARX, *), AMATRX(NDOFEL, NDOFEL), + SVARS(NSVARS), ENERGY (8), PROPS $(*), \operatorname{COORDS}($ MCRD, NNODE),

$+\mathrm{U}(\mathrm{NDOFEL}), \mathrm{DU}\left(\mathrm{MLVARX},{ }^{*}\right), \mathrm{V}(\mathrm{NDOFEL}), \mathrm{A}(\mathrm{NDOFEL}), \operatorname{TIME}(2)$,

$+\operatorname{PARAMS}(*)$, JDLTYP(MDLOAD, *), ADLMAG(MDLOAD, *),

$+\operatorname{DDLMAG}(\operatorname{MDLOAD}, *), \operatorname{PREDEF}(2, \operatorname{NPREDF}, \operatorname{NNODE}), \operatorname{LFLAGS}(*)$,

$+\operatorname{JPROPS}(*)$

jtype $=3$

NJPROP $=1$

$\operatorname{JPROPS}(1)=1$

return

end 


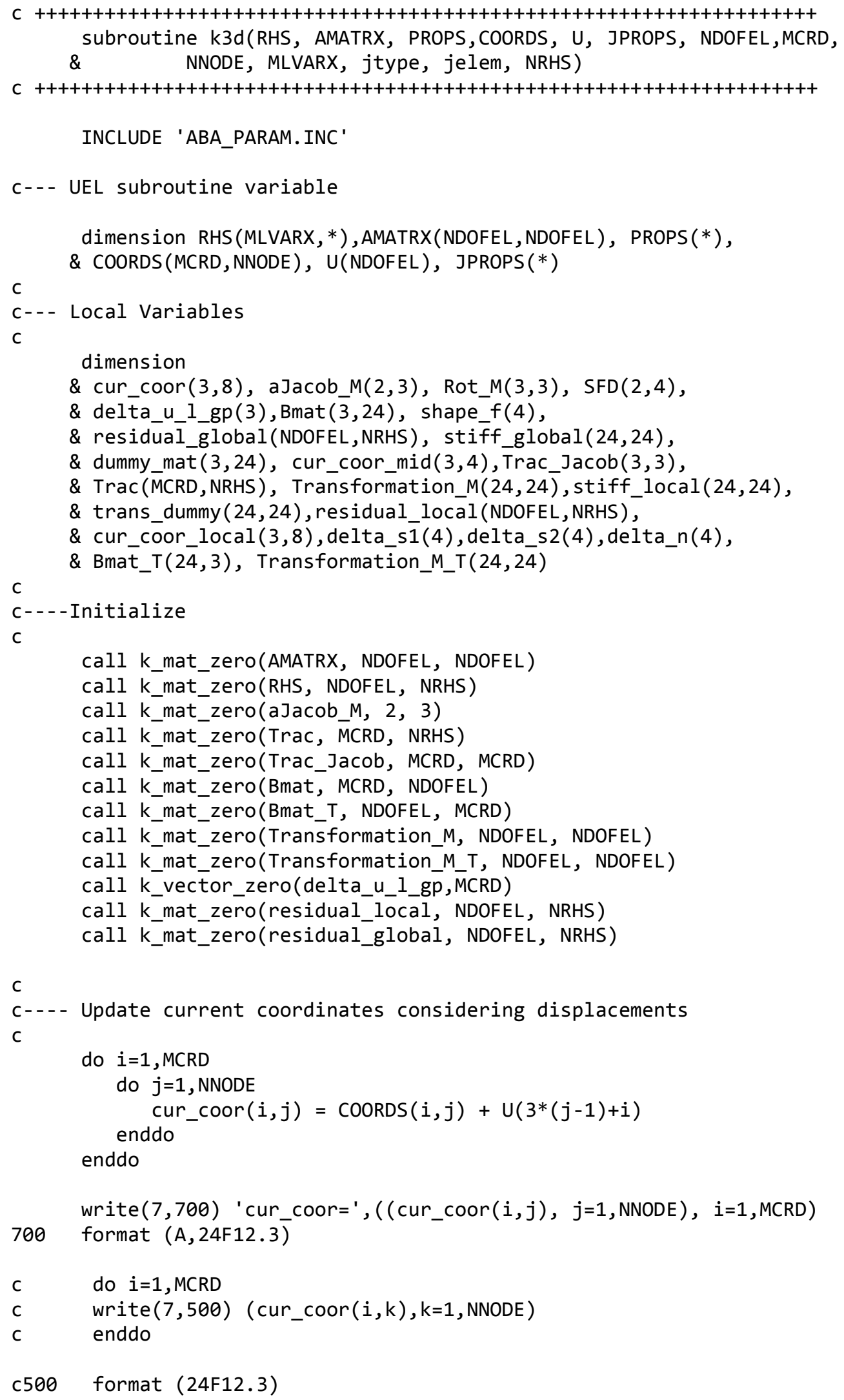




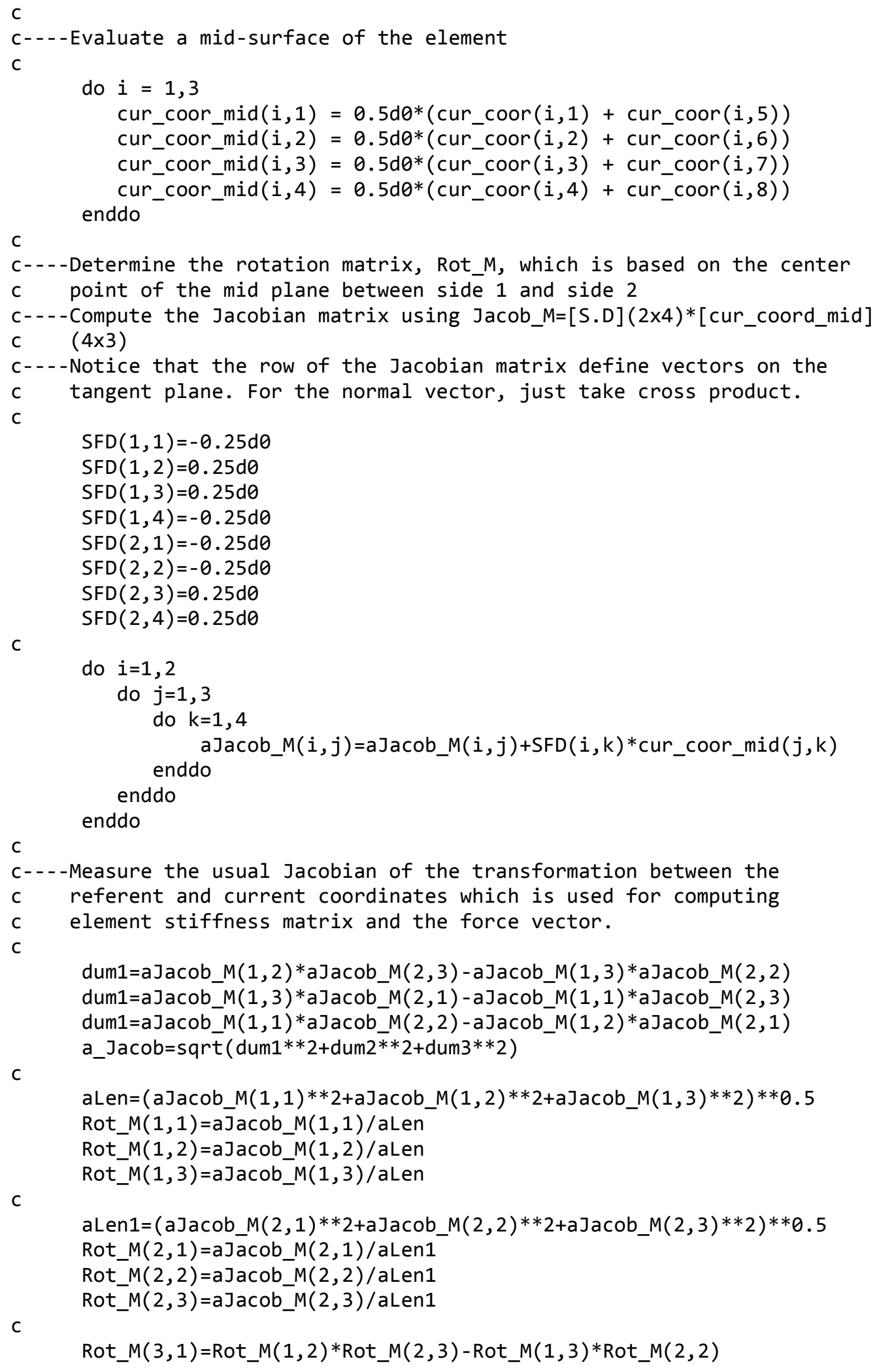




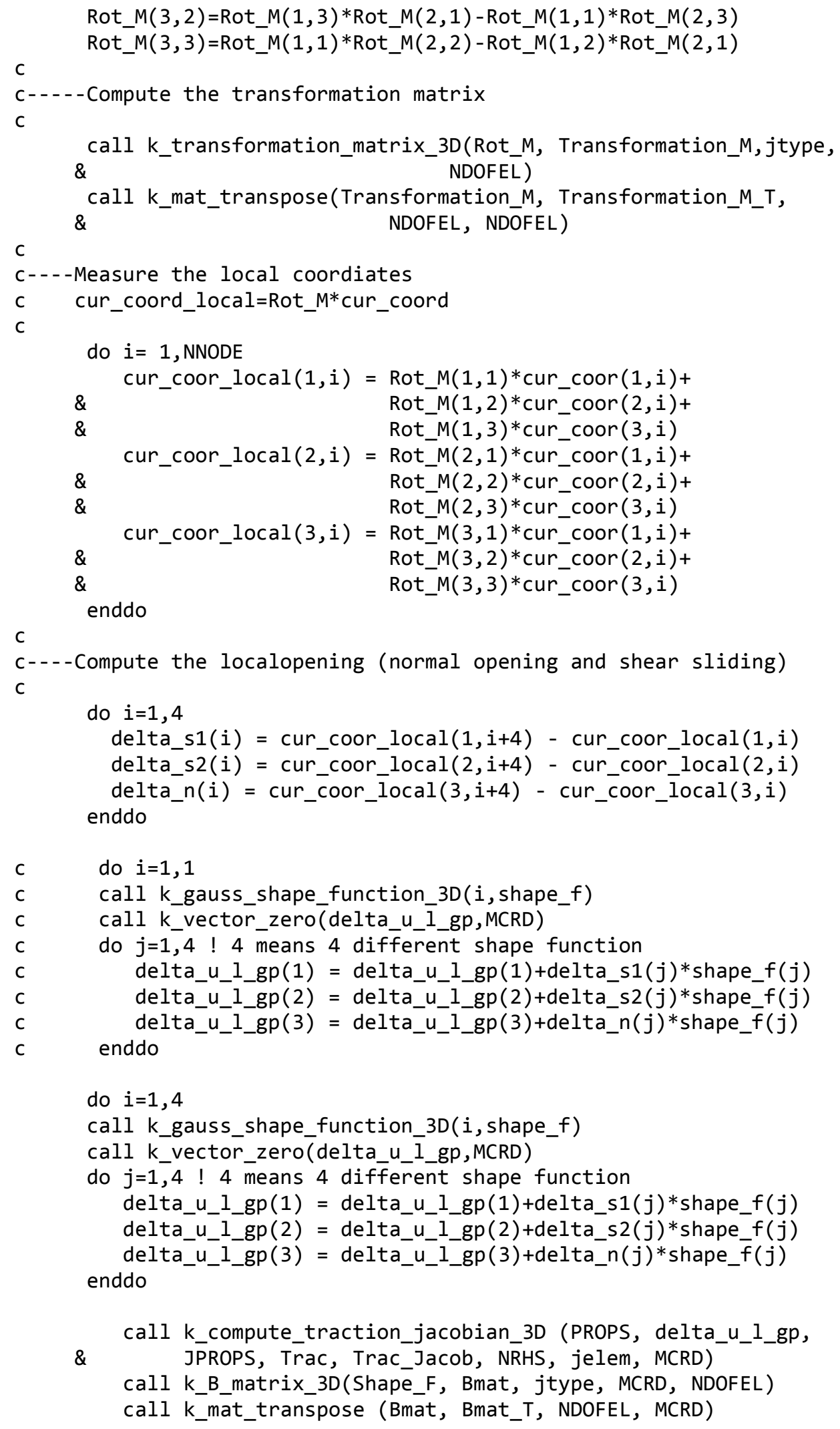




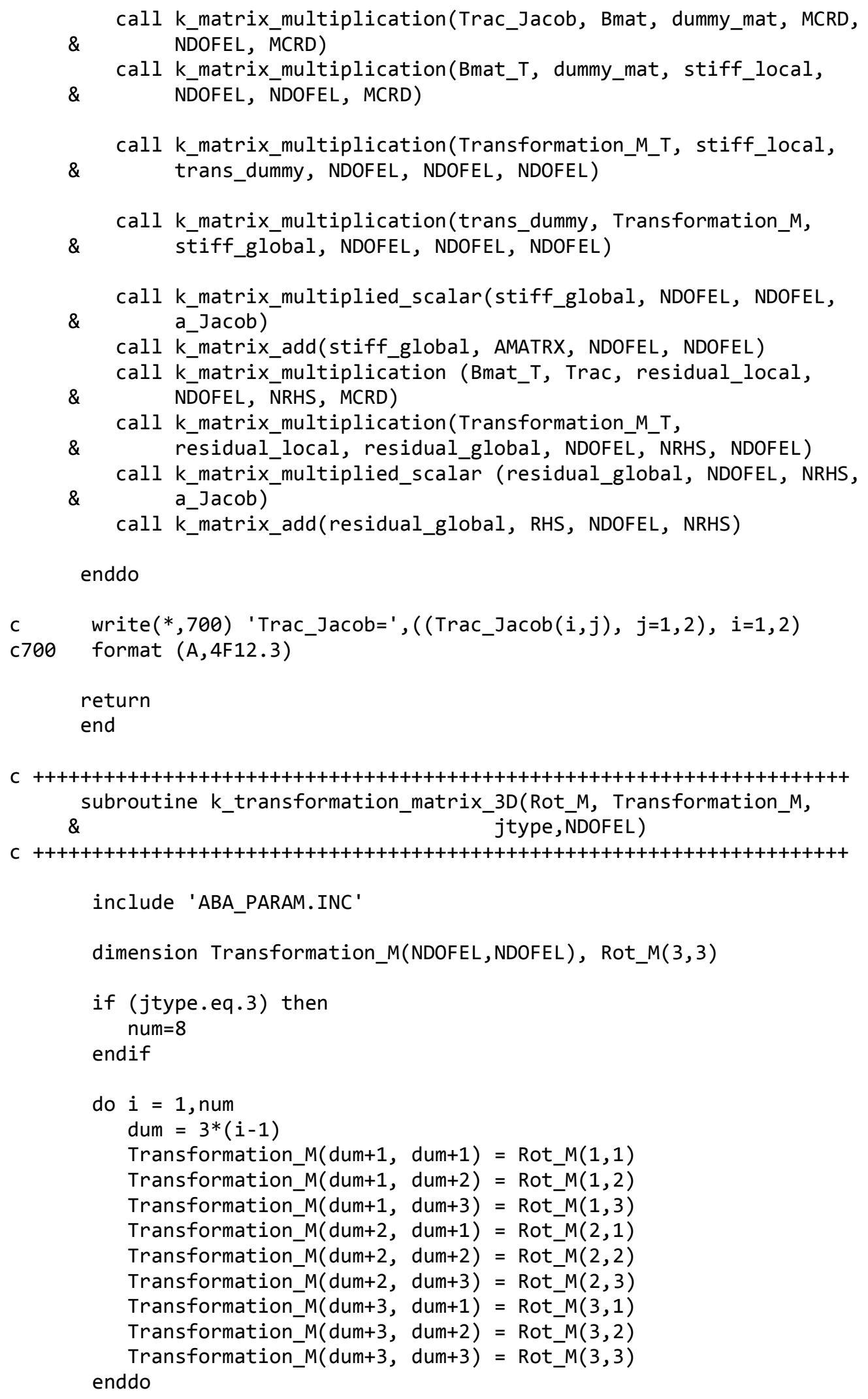




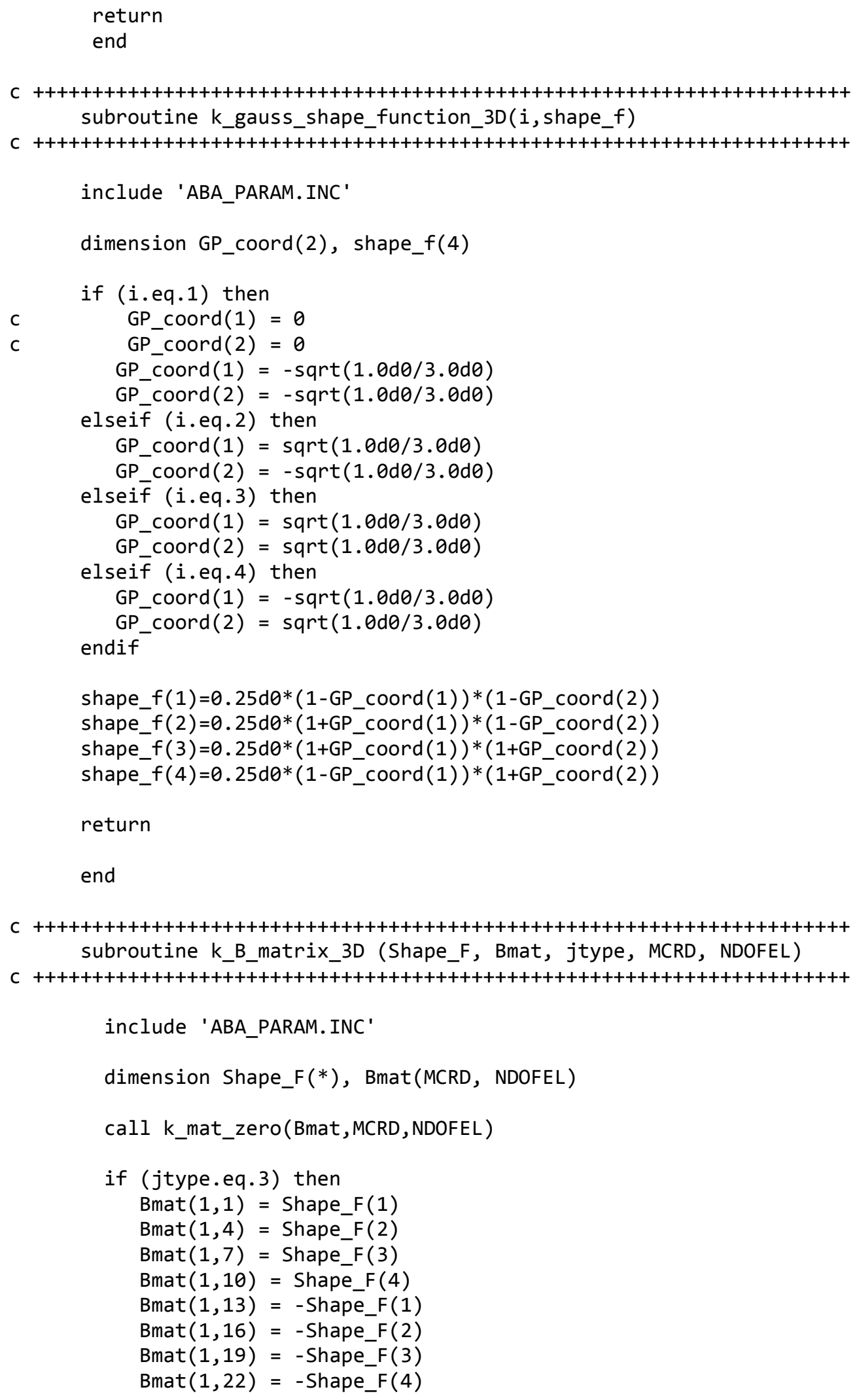




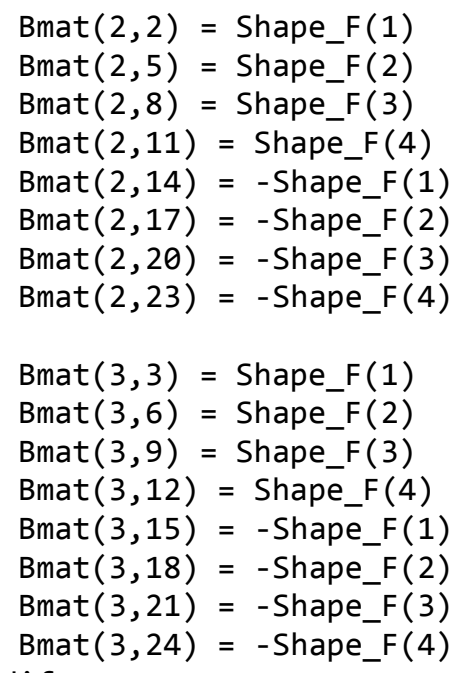


del_c $=$ del_c_new

del_cc $=$ del__c*ambda_cr

endif

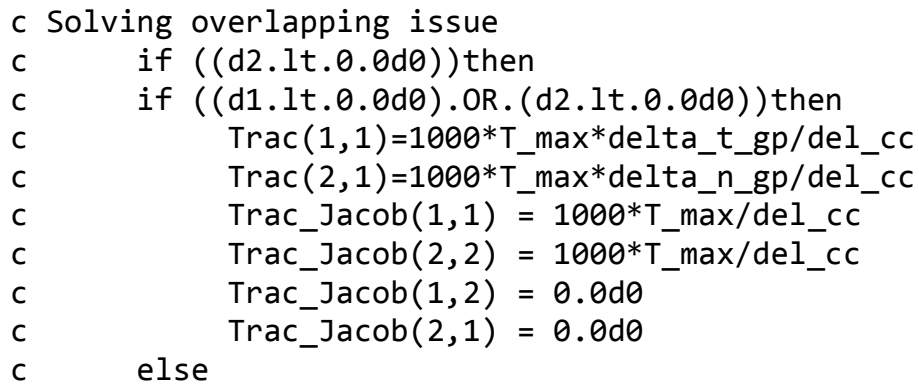

if (del_eff.le.del_cc) then

$\operatorname{Trac}(1,1)=T$ max*delta_t1_gp/del_cc

$\operatorname{Trac}(2,1)=T$ _max*delta_t2_gp/del_cc

$\operatorname{Trac}(3,1)=$ T_max*delta_n_gp/del_cc

$\operatorname{Trac} J \operatorname{Jacob}(\overline{1}, 1)=\mathrm{T} \_\mathrm{max} / \mathrm{del}$ _cc

Trac_Jacob $(2,2)=T_{-} \max / \mathrm{del}_{-} \mathrm{cc}$

$\operatorname{Trac} J \operatorname{Jacob}(3,3)=\mathrm{T}_{-} \mathrm{max} / \mathrm{del} \mathbf{d}_{-} \mathrm{cc}$

Trac_Jacob $(1,2)=0.0 \mathrm{~d} \theta$

Trac_Jacob $(1,3)=0.0 \mathrm{~d} \theta$

$\operatorname{Trac} \_J a c o b(2,1)=0.0 \mathrm{~d} \theta$

Trac_Jacob $(2,3)=0.0 \mathrm{~d} \theta$

Trac_Jacob $(3,1)=0.0 \mathrm{~d} \theta$

Trac_Jacob $(3,2)=0.0 \mathrm{~d} \theta$

elseif ((del_eff.gt.del_cc).AND.(del_eff.lt.del_c)) then

$\operatorname{Trac}(1,1)=$ T_max $*(1-$ sqrt (delta_t1_gp** $2+$ delta_t2_gp**2+delta_n_gp* \& $* 2) /$ del_c)**alpha*delta_t1_gp/sqrt(delta_t1_gp**2+delta_t2_gp**2+ \& delta_n_gp**2)/(1-ambda_cr)**alpha

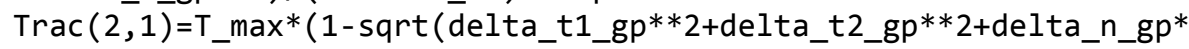

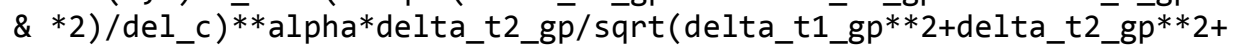
\& delta_n_gp**2)/(1-ambda_cr $) * * a l p h a$

$\operatorname{Trac}(3,1)=$ T_max* $(1-\operatorname{sqrt}($ delta_t1_gp**2+delta_t2_gp**2+delta_n_gp*

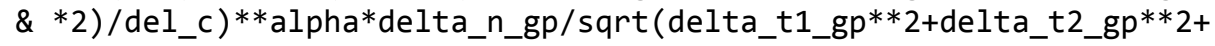
\& delta_n_gp**2)/(1-ambda_cr $) * * a l p h a$

a1 $=-T$ max*(1-sqrt (delta_t1_gp**2+delta_t2_gp**2+delta_n_gp** \&2)/del_c)**alpha*alpha/(dèlta_t1_gp**2+dèlta_t2_gp**2+dèlta_n_gp** \&2)/del_c*delta_t1_gp**2/(1-sqrt (delta_t1_gp**2+delta_t2_gp**2+delt \&a_n_gp**2)/del_c)/(1-ambda_cr)**alpha+T_max*(1-sqrt (delta_t1_gp**2 \&+deltta_t2_gp**2+delta_n_gp**2)/del_c)**alpha/sqrt (delta_t1_gp**2+d \&elta_t2_gp**2+delta_n_gp**2)/(1-ambda_cr)**alpha-T_max*(1-sqrt (del \&ta_t1_gp**2+delta_t2_gp**2+delta_n_gp**2)/del_c)**alpha*delta_t1_g \&p**2/sqrt (delta_t1_gp**2+delta_t2_gp**2+delta_n_gp**2)**3/(1-ambda \&_cr)**alpha

a2 = -T_max*(1-sqrt (delta_t1_gp**2+delta_t2_gp**2+delta_n_gp**

\&2)/del_c)**alpha*alpha/(delta_t1_gp**2+delta_t2_gp**2+delta_n_gp**

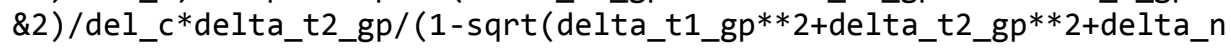


\& gp**2)/del_c)*delta_t1_gp/(1-ambda_cr)**alpha-T_max*(1-sqrt(delta \&_t1_gp**2+delta_t2_gp**2+delta_n_gp**2)/del_c)**alpha*delta_t1_gp/

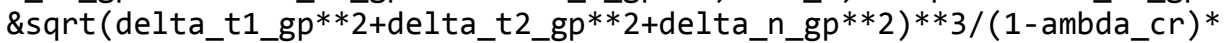
\&*alpha*delta_t2_gp

a3 = -T_max*(1-sqrt (delta_t1_gp**2+delta_t2_gp**2+delta_n_gp**

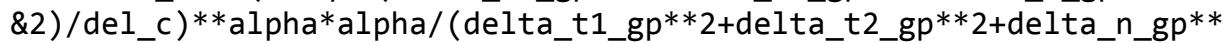
\&2)/del_c*delta_n_gp/(1-sqrt (dēta_t1_gp**2+delta_t2_gp**2+delta_n_ \&gp**2)/del_c)*delta_t1_gp/(1-ambda_cr)**alpha-T_max*(1-sqrt(delta_ \&t1_gp**2+delta_t2_gp**2+delta_n_gp**2)/del_c)**alpha*delta_t1_gp/s \&qrt (delta_t1_gp**2+delta_t2_gp**2+delta_n_gp**2)**3/(1-ambda_cr)** \&alpha*delta_n_gp

a4 = -T_max*(1-sqrt (delta_t1_gp**2+delta_t2_gp**2+delta_n_gp**

\&2)/del_ç $* * a l p h a * a l p h a /($ delta_t1_gp**2+dèlta_t2_gp**2+dèlta_n_gp** \&2)/del_c*delta_t2_gp**2/(1-sqrt (delta_t1_gp**'2+delta_t2_gp** $2+$ delt \&a_n_gp**2)/del_c)/(1-ambda_cr)**alpha+T_max*(1-sqrt(delta_t1_gp**2 \&+delta_t2_gp**2+delta_n_gp**2)/del_c)**alpha/sqrt (delta_t1__gp**2+d \&elta_t2_gp**2+delta_n_gp**2)/(1-ambda_cr)**alpha-T_max*(1-sqrt(del \&ta_t1_gp**2+delta_t2_gp**2+delta_n_gp**2)/del_c)**alpha*delta_t2_g \&p**2/sqrt (delta_t1__gp**2+delta_t2_gp**2+delta_n_gp**2)**3/(1-ambda \&_cr)**alpha

a5 = -T_max*(1-sqrt (delta_t1_gp**2+delta_t2_gp**2+delta_n_gp**

\&2)/del_c, )**alpha*alpha/(dèlta_t1_gp**2+dèlta_t2_gp**2+dèlta_n_gp** \&2)/del_c*delta_n_gp/(1-sqrt (dēta_t1_gp**2+delta_t2_gp**2+dēta_n_ \&gp**2)/del_c)*delta_t2_gp/(1-ambda_cr)**alpha-T_max*(1-sqrt(delta_ \&t1_gp**2+delta_t2_gp**2+delta_n_gp**2)/del_c)**alpha*delta_t2_gp/s \&qrt (delta_t1_gp $* * 2+$ delta_t2_gp $\left.* * 2+d e l t a \_n \_g p * * 2\right) * * 3 /\left(1-a m b \bar{d} a \_c r\right) * *$ \&alpha*delta_n_gp

a6 = -T_max*(1-sqrt (delta_t1_gp**2+delta_t2_gp**2+delta_n_gp**

\&2)/del_c)**alpha*alpha/ (delta_t1_gp**2+delta_t2_gp**2+delta_n_gp**

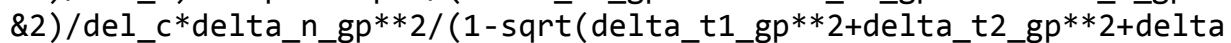
\& n gp**2)/del $\bar{c}) /(1-a m b d a ~ c r) * * a l p h a+T \max *(1-\operatorname{sqrt}(\overline{d e l t a}$ t1 gp**2+ \&delta_t2_gp**2+delta_n_gp**2)/del_c)**alpha/sqrt (delta_t1__gp**2+de \&lta_t2_gp**2+delta_n_gp**2)/(1-ambda_cr)**alpha-T_max*(1-sqrt (delt \&a_t1_gp**2+delta_t2_gp**2+delta_n_gp**2)/del_c)**alpha*delta_n_gp* $\& * \overline{2} /$ sqrt (delta_t1_gp**2+delta_t2_gp**2+delta_n̄_gp $* * 2) * * 3 /(1-a \overline{m b} \overline{d a}$ _c $\& r) * * a l p h a$

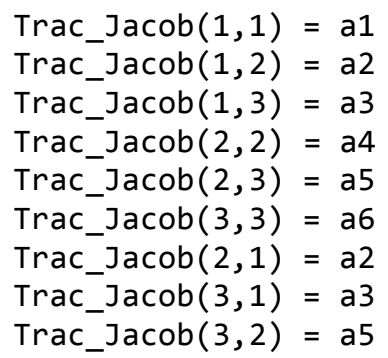

elseif (del_eff.gt.del_c) then

$\operatorname{Trac}(1, \overline{1})=0.0 \mathrm{~d} \theta$

$\operatorname{Trac}(2,1)=0.0 \mathrm{~d} \theta$

$\operatorname{Trac}(3,1)=0.0 \mathrm{~d} 0$

$\operatorname{Trac} \_\operatorname{Jacob}(1,1)=0.0 \mathrm{~d} \theta$ 


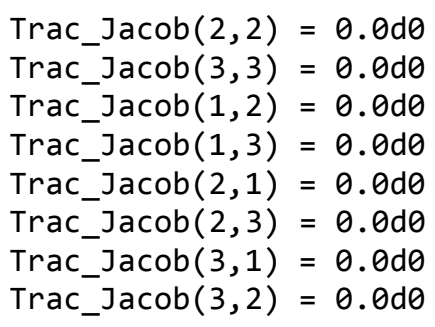

C +++++++++++++++++++++++++++++++++++++++++++++++++++++++++++++++++++++

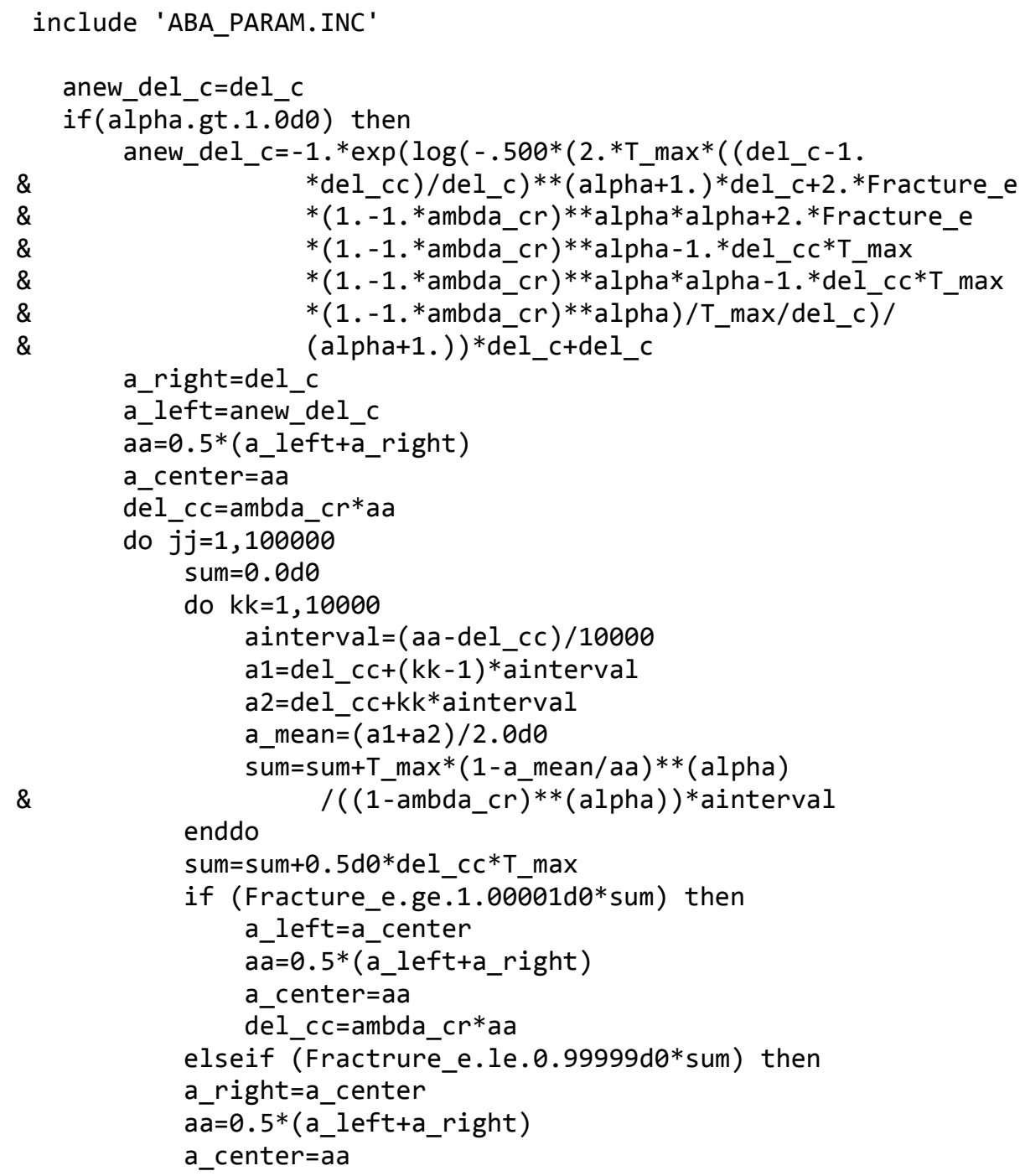




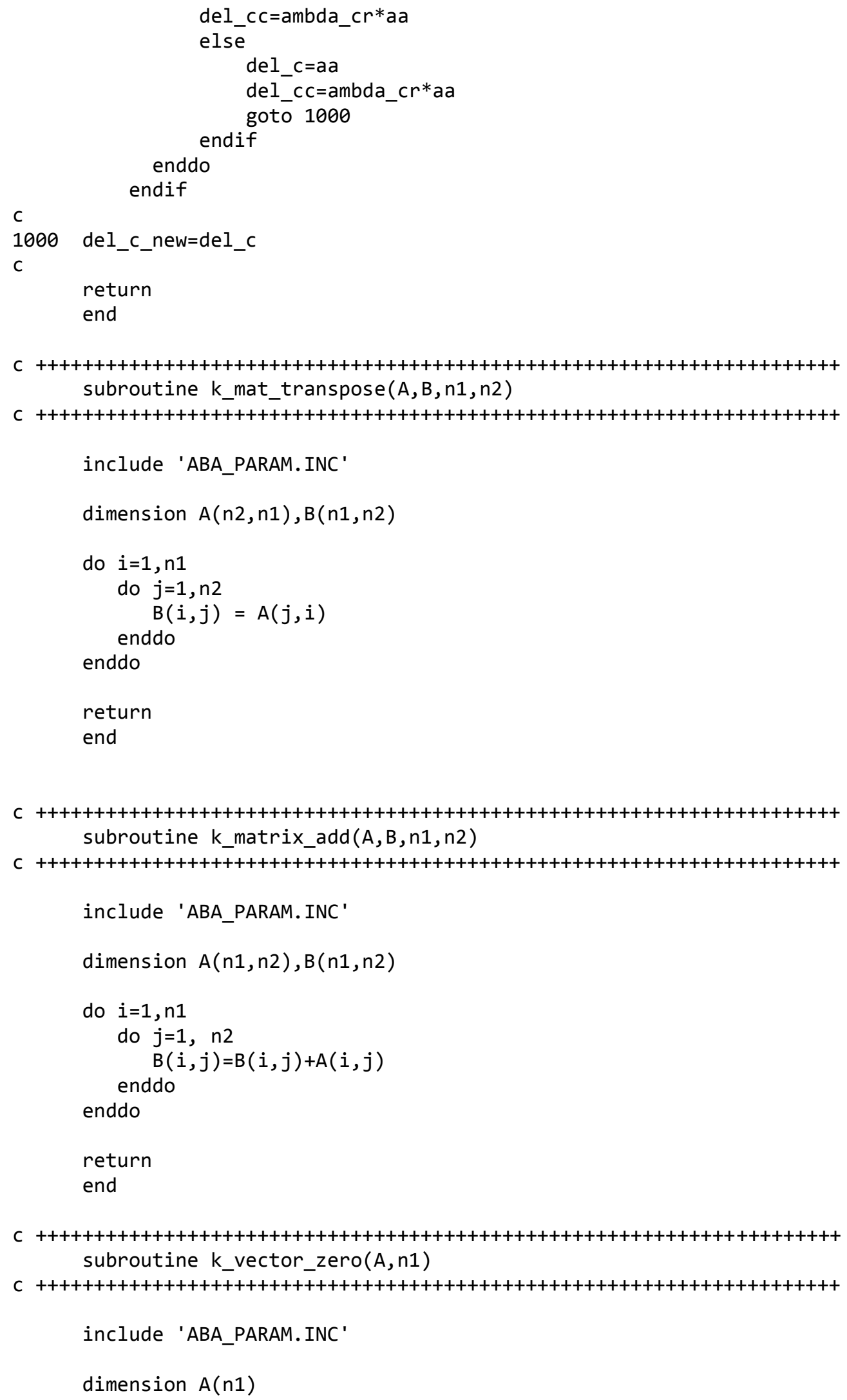


do $i=1, n 1$

$A(i)=0.0 \mathrm{~d} \theta$

enddo

return

end

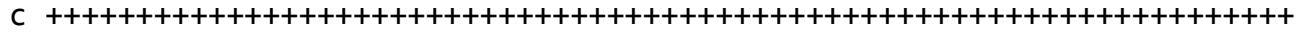
subroutine k_matrix_multiplication $(A, B, C, n 1, n 2, n 3)$

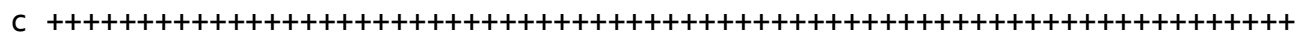

include 'ABA_PARAM. INC'

dimension $A(n 1, n 3), B(n 3, n 2), C(n 1, n 2)$

call k_mat_zero(c,n1, n2)

do $i=1, n 1$

do $j=1, n 2$

do $k=1, n 3$

$C(i, j)=C(i, j)+A(i, k) * B(k, j)$ enddo

enddo

enddo

return

end

C +++++++++++++++++++++++++++++++++++++++++++++++++++++++++++++++++++++ subroutine k_matrix_multiplied_scalar(A, n1,n2,fac)

C +++++++++++++++++++++++++++++++++++++++++++++++++++++++++++++++++++++

include 'ABA_PARAM. INC'

dimension $A(n 1, n 2)$

do $i=1, n 1$

do $j=1, n 2$

$A(i, j)=A(i, j) * f a c$

enddo

enddo

return

end

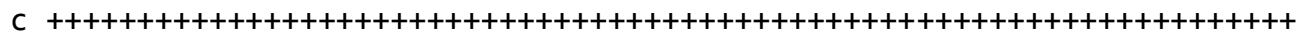
subroutine k_mat_zero $(A, n 1, n 2)$

C +++++++++++++++++++++++++++++++++++++++++++++++++++++++++++++++++++++

include 'ABA_PARAM. INC'

dimension $A(n 1, n 2)$

do $i=1, n 1$ 


$$
\begin{array}{ll} 
& \text { do } j=1, n 2 \\
& A(i, j)=0.0 \text { d0 } \\
& \text { enddo } \\
& \text { enddo } \\
& \text { return } \\
& \text { end } \\
\text { c } & \text { write(*,400) jelem } \\
\text { c400 } & \text { format }(F 5.3)
\end{array}
$$


Appendix C: Documents of permission to use copyrighted materials 


\section{Appendix C1: Copyright permission of journal titled: "Investigation of fracture behavior of heterogeneous infrastructure materials with extended-finite-element method and image anlaysis"}

List of applied figure(s): Figure 2-7

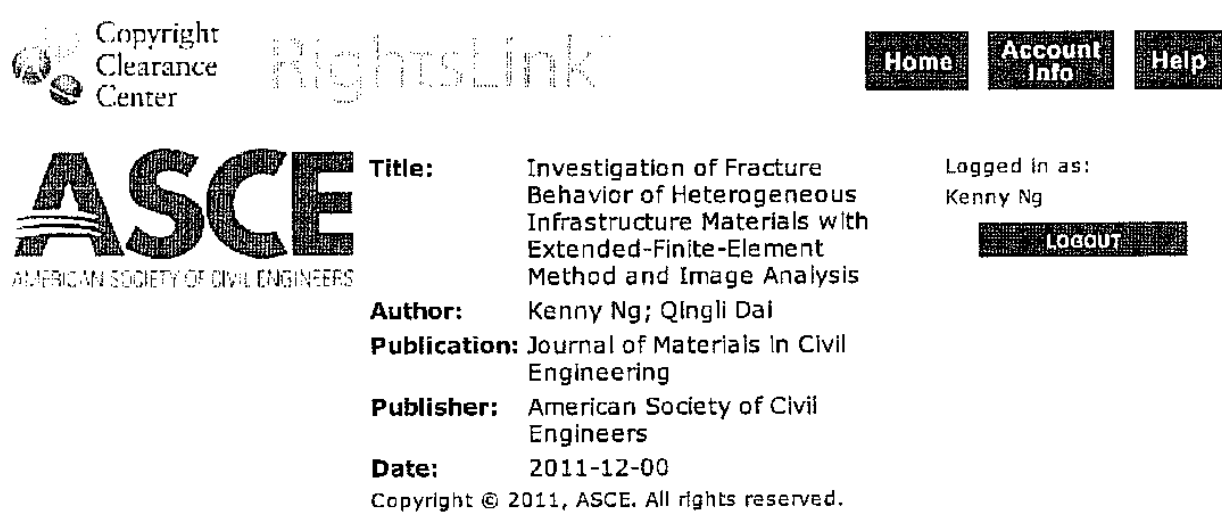

Permissions Request

As an ASCE author, you are permitted to reuse you own content for another ASCE or non-ASCE publication.

Please add the full credit line "With permission from ASCE" to your source citation. Please print this page for your records.

Type of use: Dissertation/Thesis

Portion: figures/tables/illustrations

Format: print and electronic

Use of this content will make up more than $25 \%$ of the new work: no

Author of this ASCE work or ASCE will publish the new work: yes

\section{BACK Cosewlipow}

Copyright (c) 2012 Copyright Clearance Center. Inc. All Rights Reserved. Privacy statement. Comments? We would llke to hear from you. E-mail us at customercare@copyriaht.com 


\section{Appendix C2: Copyright permission of journal titled "Concrete fracture properties with compact tension test"}

List of applied figure(s): Figure 2-1, Figure 2-2, and Figure 2-6.

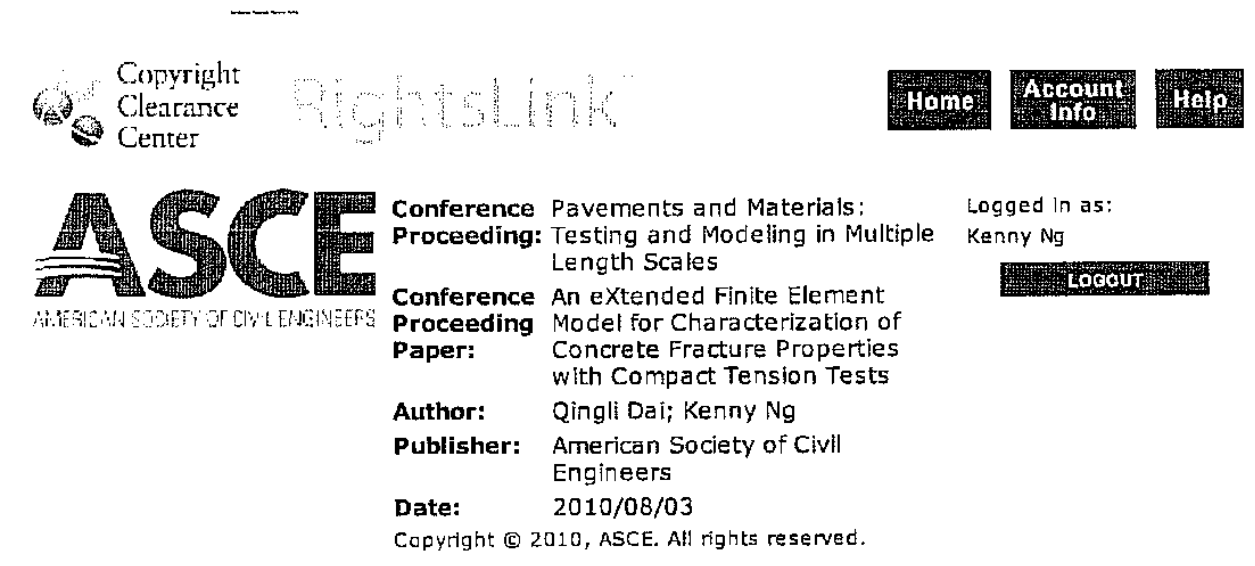

Permissions Request

As an ASCE author, you are permitted to reuse you own content for another ASCE or non-ASCE publication.

Please add the full credit line "With permission from ASCE" to your source citation. Please print this page for your records.

Type of use: Dissertation/Thesis

Portion: figures/tables/iliustrations

Format: electronic

Use of this content will make up more than $25 \%$ of the new work: no

Author of this ASCE work or ASCE will publish the new work: yes

\section{H BACK}

Copyright (c) 2012 Copuriaht Clearance Center, Inc. All Rights Reserved. Privacy statement. Comments? We would like to hear from you. E-mall us at gustomercare @ecopyriaht.com 


\section{Appendix C3: Copyright permission of journal titled "Tailored extended finite- element model for predicting crack propagation and fracture properties within idealized and digital cementitious material samples"}

List of applied figure(s): Figure 2-1, Figure 2-2, Figure 2-3, Figure 2-4, Figure 2-5, Figure 2-6, Figure 2-8, Figure 2-9, Figure 2-10, Figure 2-11, Figure 3-5, Figure 4-1, Figure 4-2, and Figure 6-1

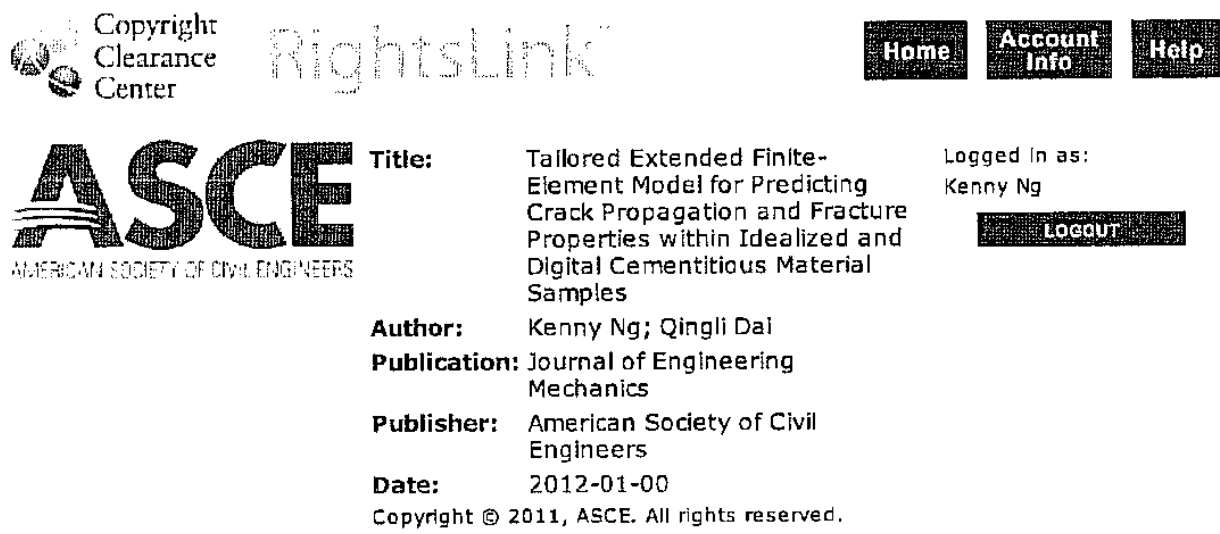

Permissions Request

As an ASCE author, you are permitted to reuse you own content for another ASCE or non-ASCE publication.

Please add the full credit line "With permission from ASCE" to your source citation. Please print this page for your records.

Type of use: Dissertation/Thesis

Portion: figures/tables/illustrations

Format: electronic

Use of this content will make up more than $25 \%$ of the new work: no

Author of this ASCE work or ASCE will publish the new work: yes

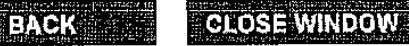

Copyrigt (c) 2012 Copyricht Clearance Center. Inc. All Rights Reserved. Privacy statement. Comments? We would like to hear from you. E-mail us at customercare@copyright.com 


\title{
Appendix C4: Copyright permission of book titled "Concrete: microstructure, properties, and materials"
}

List of Figure(s) applied: Figure 1-1

$10 / 9 / 12$

Copyright Clearance Canter

\author{
Coprotight \\ W. Conterince
}

Confirmation Number: 11033803

Order Date: $10 / 03 / 2012$

Customer Information

Customer: Kenny $\mathrm{Ng}$

Account Number: 3000576378

Organization: Kenny Ng

Email: keng@mtu.edu

Payment Method: Credit Card ending in

5189

Order Details

Concrete : microstructure, properties, and materials

\begin{tabular}{|c|c|}
\hline Order detail ID: & 62972375 \\
\hline $\begin{array}{l}\text { ISBN: } \\
\text { Publication year: }\end{array}$ & $\begin{array}{l}978-0-07-146289-1 \\
2005\end{array}$ \\
\hline $\begin{array}{l}\text { Publication } \\
\text { Type: }\end{array}$ & Book \\
\hline Publisher: & MCGRAW-HILL \\
\hline Rightsholder: & $\begin{array}{l}\text { MCGRAW-HILL COMPANIES, INC, - } \\
\text { BOOKS }\end{array}$ \\
\hline Author/Editor: & $\begin{array}{l}\text { P. Kumar Mehta AND Paulo J.M. } \\
\text { Montelro }\end{array}$ \\
\hline
\end{tabular}

Permission Status: Granted

Comment: Permission granted provided figure is not cited to another source.

Permission type: Republish or dlsplay content

Type of use: Dissertatlon

Requested use: D/ssertation

Requested use: Dlssertation
Republication Computational and experimental

Republication Computational and experirnental
title:

title: $\quad \begin{array}{ll}\text { Investigation of Internal frost } \\ \text { of cement-based materiats }\end{array}$

Republishing Michigan Technological University

organization:

Organization Non-profit 501(c)/3)

status:

Republication 12/24/2012

date:

Circulation/

Distribution:

Type of content: Figure/dlagram/table

Description of Concrete : microstructure, properties,

requested

and materlals - Figure 2-7

Page range(s): 3

Translating to: No Translation

Requested 09/26/2005

content's

publication date:

Payment

CC ending in $\mathbf{5 1 8 9}$ 


\section{Appendix C5: Copyright permission of journal titled "A review of salt scaling: II. Mechanisms"}

List of Figure(s) applied: Figure 1-3

\section{ELSEVIER LICENSE TERMS AND CONDITIONS}

Oct 09,2012

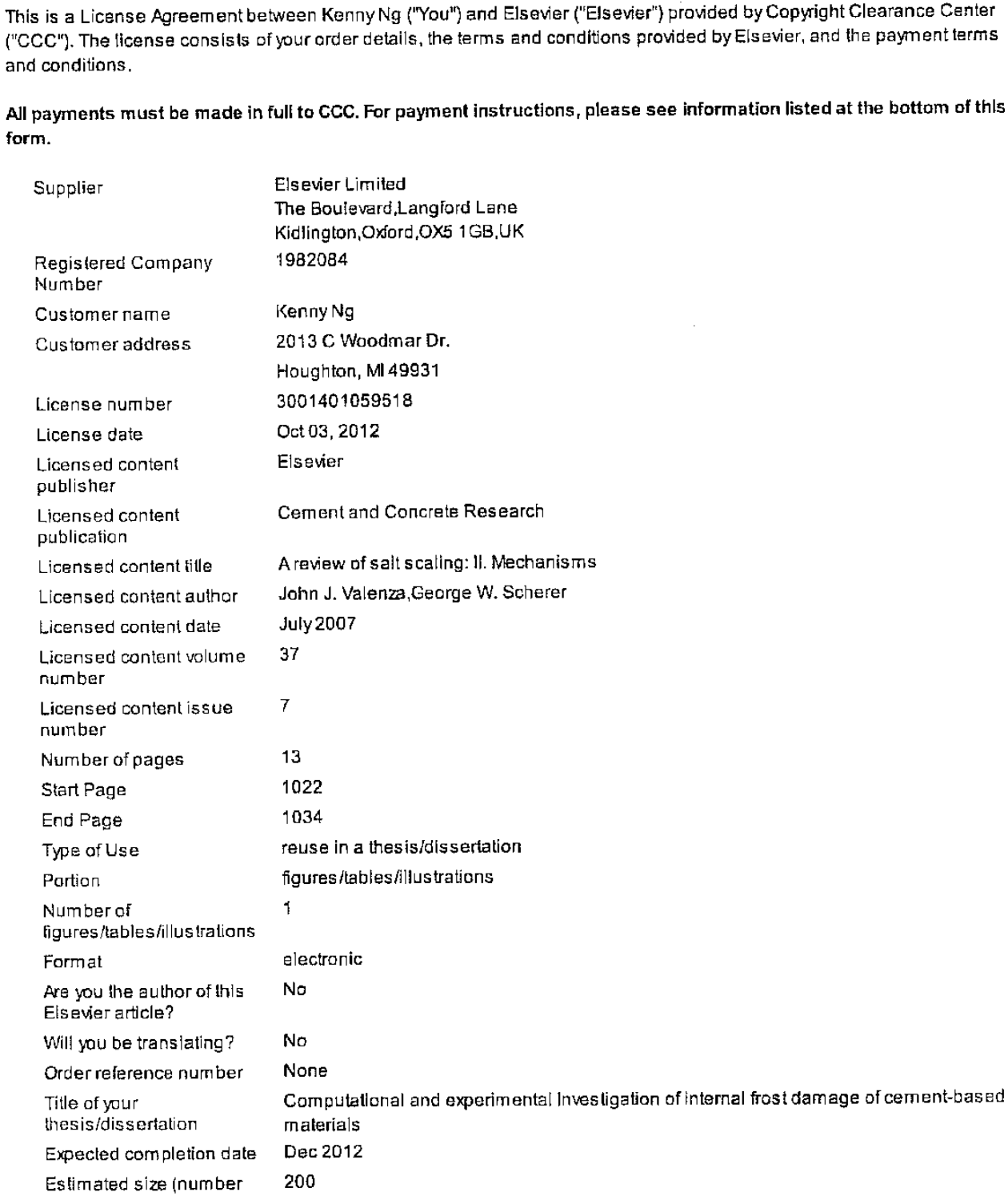

All payments must be made in full to CCC. For payment instructions, please see information listed at the bottom of thls form.

Supplier

Register

Number

Customer name

Customer address

License number

License date

Licensed content

publisher

Licensed content

publication

Licensed content fille

Licensed content author

Licensed content date

Licensed content volume

number

Licensed content issue

number

Number of pages

Start Page

End Page

Type of Use

Portion

Number of

figures/tables/illus trations

Format

Are you the author of this

Els evier article?

Will you be translating?

Order reference number

Title of your

thesis/dissertation

Expecled completion date

Estimated size (number 
of pages)

Elsevier VAT number $\quad$ GB 494627212

Pemissions price $\quad 0.00$ USD

VAT/Local Sales Tax $\quad 0.0$ USD / 0.0 GBP

Total $\quad 0.00$ USD

Terms and Conditions

\section{INTRODUCTION}

1. The publisher for this copyrighted material is Elsevier. By clicking "accept" in connection with completing this licensing transaction, you agree that the following terms and conditions apply to this transaction (along with the Billing and Payment terms and conditions established by Copyright Clearance Center, Inc. ("CCC"), at the time that you opened your

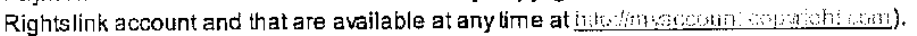

\section{GENERAL TERMS}

2. Elsevier hereby grants you permission to reproduce the aforementioned material subject to the terms and conditions indicated.

3. Acknowledgement: If any part of the material to be used (for example, figures) has appeared in our publication with credit or acknowledgement to another source, permission must also be sought from that source. If such permission is not obtained then that material may not be included in your publication/copies. Suitable acknowledgement to the source must be made, either as a footnote or in a reference list at the end of your publication, as follows:

"Reprinted from Publication title, Vol /edition number, Author(s), Titie of article/title of chapter. Pages No., Copyright (Year), with permission from Elsevier [OR APPLICABLE SOCIETY COPYRIGHT OWNER]." Asso Lancet speclal credit "Reprinted from The Lancet, Vol. number, Author(s), Title of article. Pages No.. Copyright (Year), with permis sion from Elsevier."

4. Reproduction of this material is confined to the purpose and/or media for which permission is hereby given.

5. Atering/Modifying Material: Not Permitted. However figures and illustrations may be alterediadapted minimally to serve your work. Any other abbreviations, additions, deletions and/or any other alterations shall be made only with prior written authorization of Els evier Ltd. (Please contact Elsevier at permissions@elsevier.com)

6 . If the permission fee for the requested use of our material is walved in this instance, please be advised that your future requests for Els evier materials mayattract a fee.

7. Reservation of Rights: Publisher reserves all rights not specifically granted in the combination of (i) the license detalis provided by you and accepted in the course of this licensing transaction, (ii) these terms and conditions and (iii) CCC's Billing and Payment terms and conditions.

8. License Contingent Upon Payment: While you may exercise the rights licensed immedialely upon issuance of the license at the end of the licensing process for the transaction, provided that you have disclosed complete and accurate details of your proposed use, no license is finally effective unless and until full payment is received from you (either by publisher or by CCC) as provided in CCC's Billing and Payment terms and condltions. If full payment is not received on a timely basis, then any license preliminarily granted shall be deemed automatically revoked and shall be wid as if never granted. Further, in the event that you breach any of these terms and conditions or any of CCC's Billing and Payment terms and conditions, the license is automatically revoked and shall be void as if never granted. Use of materials as described in a revoked license, as well as any use of the materials beyond the scope of an urvevoked license, may constitute copyright infringement and publisher reserves the right to take any and all action to protect its copyright in the materials.

9. Warranties: Publlsher makes no representations or warranties with respect to the licensed material.

10. Indemnity. You hereby indemnify and agree to hold harmless publisher and CCC, and their respective officers, directors, employees and agents, from and against any and all claims arising out of your use of the licensed material other than as specifically authorized pursuant to this license.

11. No Transfer of License: This license is personal to you and may not be sublicensed, assigned, or transferred by you to any other person without publisher's written permission.

12. No Amendment Except in Writing: This license may not be amended except in a writing signed by both parties (or, in the case of publisher, by CCC on publisher's behalf).

13. Objection to Contrary Terms: Publisher hereby objects to any terms contained in any purchase order. acknowledgment, check endorsement or other writing prepared by you, which terms are inconsis tent with these terms and conditions or CCC's Billing and Payment terms and conditions. These terms and conditions, together with CCC's Billing and Payment terms and conditions (which are incorporated herein), comprise the entire agreement between you and publisher (and CCC) concerning this licensing transaction. In the event of any confict between your obligations established by these ferms and condltions and those es tablished by CCC's Billing and Payment terms and conditions. 
these terms and conditions shall control.

14. Revocation: Elsevier or Copyright Clearance Center may deny the permissions described in this License at their sole discretion, for any reas on or no raason, with a full refund payable to you. Notice of such denial will be made using the contact information provided by you. Failure to receive such notice will not alter or invalidate the denial. In no event will Els evier or Copyright Clearance Center be responsible or liable for any costs, expenses or damage incurred by you as a result of a denlal of your permiss lon request, other than a refund of the amounl(s) paid by you to Elsevier and/or Copyright Clearance Center for denied permissions.

\section{LIMITED LICENSE}

The following terms and conditions apply only to speclfic license types;

15. Translation: This permission is granted for non-exclusive world English rights only unless your license was granted for transiation rights. If you licensed translation rights you may only translate this content into the languages you requested. A professional transiator must periorm all translations and reproduce the content word for ward preserving the integrity of the arlicle. If this license is to re-use 1 or 2 figures then permission is granted for non-excluslve world rights in all languages.

16. Website: The following terms and conditions apply to electronlc reserve and author websites:

Eectronic reserve: If licensed material is to be posted to website, the web site is to be password-protected and made available only to bona ide students registered on a relevanl course if:

This license was made in connection with a course.

This permission is granted for 1 year only. You may abtain a license for future website posting.

All content posted to the web site must maintain the copyright information line on the bottom of each image

A hyper-text must be included to the Homepage of the journal from which you are licensing at

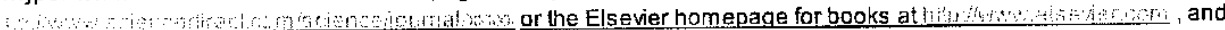
Central Storage: This license does not include permission for a scanned version of the material to be stored in a central repository such as that provided by Heron/XanEdu.

17. Author website for journals with the following additional ciauses:

Al content posted to the web site must maintain the copyright information line on the bottom of each image, and the permission granted is limited to the personal version of your paper. You are not allowed to download and post the publis hed electronic version of your article (whether PDF or HTML, proof or final version). nor may you scan the printed edition to create an electronic version. A hyper-text must be included to the Homepage of the Joumal from which you are licensing at an e-mail notice when your article appears on Elsevier's online service SclenceDirect (www.sciencedirect.com). Thatemail will inciude the articie's Digital Object Identifier (DOI). This number provides the electronic link to the published article and should be included in the posting of your personal version. We ask that you wait until you receive this e-mail and have the DOl to do any posting.

Central Storage: This license does not include permission for a scanned version of the material to be stored in a central repository such as that provided by Heron/XanEdu.

18. Author website for books with the following additional clauses:

Authors are permitted to place a brief summary of their work online oniy.

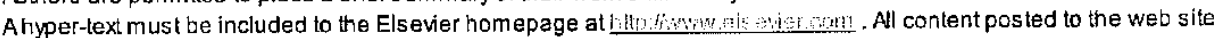
must maintain the copyright information Ine on the bottom of each inage. You are not allowed to download and post the publis hed electronic version of your chapter, nor may you scan the printed edition to create an electronic version.

Central Storage: This license does not include permission for a scanned vers ion of the material to be stored in a central repository such as that provided by Heron/XanEdu.

19. Website (regular and for author): A hyper-text must be included to the Homepage of the journal from which you are

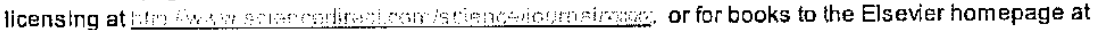

http://www.els evier.com

20. Thesis/Dissertation: If your license is for use in a thesis/dissertation your thesis may be submitted to your ins titution in elther print or electronic form. Should your thes is be published commercially, please reapply for permission. These requirements include permis sion for the Library and Archives of Canada to supply single copies, on demand, of the complete thesis and include permission for UMl to supply single coples, on demand, of the complete thesis. Should your thes is be published commercially, please reapply for permission.

21. Other Conditions:

v 1.6

If you would like to pay for this license now, please remit this license along with your payment made payable to "COPYRIGAT CLEARANCE CENTER" otherwise you will be invoiced within $\mathbf{4 8}$ hours of the license date. Payment should 
be in the form of a check or money order referencing your account number and this invoice number RLNK500869762. Once you receive your invoice for this orcier, you may pay your invoice by credit card. Please follow instructions provided at that time.

Make Payment To:

Copyright Clearance Center

Dept 001

P.O. Box 843006

Bostor, MA 02284-3006

For suggestions or comments regarding this order, contact RightsLink Customer Support:

Ut

Gratis licenses (referencing $\$ 0$ in the Total field) are free. Please retain this printable license for your reference. No payment is required. 


\title{
Appendix C6: Copyright permission of journal titled "Damage investigation of single-edge notched beam tests with normal strength concrete and ultra high performance concrete specimens using acoustic emission techniques"
}

List of Figure(s) applied: Figure 3-4

\section{ELSEVIER LICENSE TERMS AND CONDITIONS}

\begin{abstract}
This is a License Agreement between Kenny Ng ("You") and Elsevier ("Elsevier") provided by Copyright Clearance Center ("CCC"). The license consists of your order details, the ferms and conditions provided by Elsevier, and the payment terms and conditions.
\end{abstract}

All payments must be made in full to CCC. For payment Instructions, please see information listed at the bottom of this form.

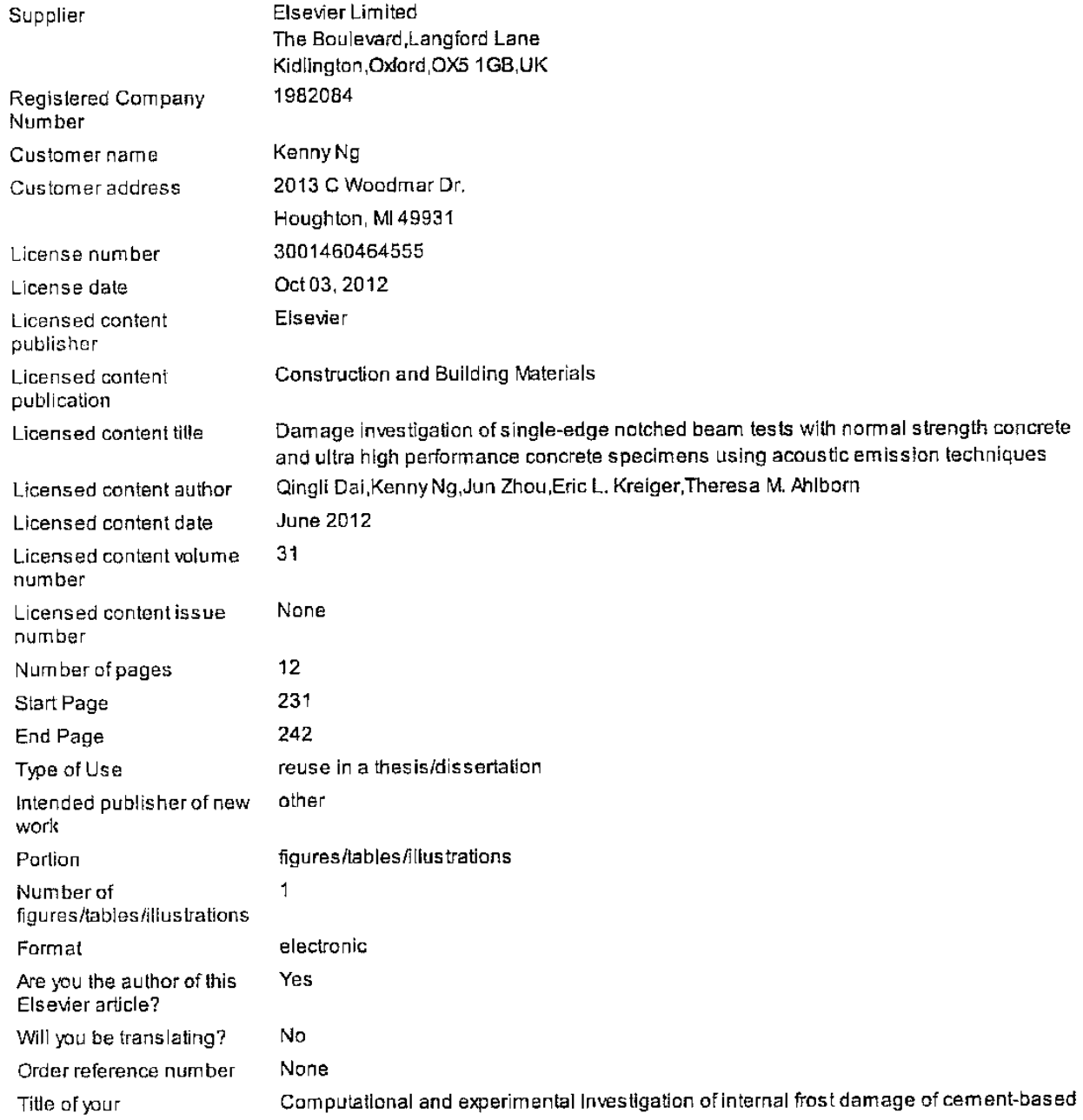




$\begin{array}{ll}\text { thesis/dissertation } & \text { materials } \\ \text { Expected completion date } & \text { Dec } 2012 \\ \begin{array}{l}\text { Estimated size (number } \\ \text { of pages) }\end{array} & 200 \\ \text { Elsevier VAT number } & \text { GB } 494627212 \\ \text { Permissions price } & 0.00 \text { USD } \\ \text { VATLocal Sales Tax } & 0.0 \text { USD / 0.0 GBP } \\ \text { Total } & 0.00 \text { USD } \\ \text { Terms and Conditions } & \end{array}$

INTRODUCTION

1. The publisher for this copyrighted material is Elsevier. Byclicking "accept" in connection with completing this licensing transaction, you agree that the following terms and conditions apply to this transaction (along with the Billing and Payment terms and conditions established by Copyright Clearance Center, Inc. ("CCC"), at the time that you opened your

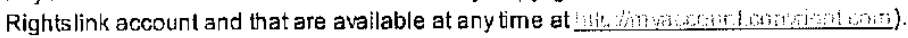

GENERAL TERMS

2. Elsevier hereby grants you permission to reproduce the aforementioned material subject to the terms and conditions indicated.

3. Acknowledgement: If any part of the material to be used (for example, figures) has appeared in our publication with credit or acknowledgement to another source, permission must also be sought from that source. If such permission is not obtained then that material may not be included in your publication/copies. Suitable acknowledgement to the source must be made, either as a footrote or in a reference list at the end of your publication, as follows:

"Reprinted from Publication title, Vol ledition number, Author(s), Title of article / title of chapter, Pages No., Copyight

(Year), with permission from Elsevier [OR APPLICABLE SOCIETY COPYRIGHT OWNER]." Also Lancet special credit "Reprinted from The Lancet, Vol. number, Author(s), Title of article, Pages No., Copyright (Year), with permission from Elsevier."

4. Reproduction of this material is confined to the purpose and/or media for which permission is hereby given.

5. Atering/Modifying Material: Not Permitted. However figures and illus trations may be altered/adapted minimally to serve your work. Any other abbreviations, additions, deletions and/or any other alterations shall be made only with prior written authorization of Els evier Ltod. (Please contact Elsevier at perm/ssions @elsevier.com)

6. If the permission fee for the requested use of our material is waived in this instance, please be advised that your future requests for Elsevier materials may attract a fee.

7. Reservation of Rights: Publisher reserves all rights not specifically granted in the combination of (i) the license details provided by you and accepted in the course of this licensing transaction, (ii) these terms and conditions and (iii) CCC's Billing and Payment terms and conditions.

8. License Contingent Upon Payment: While you may exercise the rights licensed immediately upon issuance of the license at the end of the licensing process for the transaction, provided that you have disclosed complete and accurate details of your proposed use, no license is finally effective unless and until full payment is received from you (either by publisher or by (CC) as provided in CCC's Billing and Payment terms and conditions. If full payment is not received on a timely bas is, then any license preliminarily granted shall be deemed automatically revoked and shall be void as if never granted. Further, in the event that you breach any of these terms and conditions or any of CCC's Billing and Payment terms and conditions, the license is automatically revoked and shail be void as if never granted. Use of materials as described in a revoked license, as well as any use of the materials beyond the scope of an unrevoked license, may cons tilute copyright infringement and publisher reserves the right to take any and all action to protect its copyright in the materials.

9. Warranties: Publisher makes no representations or warranties with respect to the licensed material.

10. Indemnity. You hereby indemnify and agree to hold harmless publisher and CCC, and their respective officers,

directors, employees and agents, from and against any and all claims arising out of your use of the licens ed material other than as specifically authorized purs uant to this license.

11. No Transfer of License: This ilcense is personal to you and may not be sublicensed, assigned, or transferred by you to any other person withoul publisher's written permission.

12. No Amendment Except in Wriling: This license may not be amended except in a writing signed by both parties (or. in the case of publisher, by CCC on publisher's behalf).

13. Objection to Contrary Terms: Publisher hereby objects to any terms contained in any purchase order, acknowledgment, check endorsement or ather writing prepared by you, which terms are inconsistent with these terms and conditions or CCC's Bilting and Payment terms and conditions. These terms and conditions, logether with CCC's 
Billing and Payment terms and conditions (which are incorporated herein), comprise the entire agreement between you and publisher (and CCC) concerning this Ilcensing transaction. In the event of any conflict between your obligations established by these terms and condlions and those established by CCC's Billing and Payment terms and conditions, these terms and conditions shall control.

14. Revocation: Els evier or Copyright Clearance Center may deny the permissions described in this License at their sole discretion, for any reason or no reason, with a full refund payable to you. Notice of such denial will be made using the coniact information provided by you. Failure to receive such notice will not alter or invalidate the denial. In no event will Elsevier or Copyright Clearance Cenler be responsible or liable for any costs, expenses or damage incurred by you as a result of a denial of your permission request, other than a refund of the amount(s) paid by you to Elsevier ano/ar Copyright Clearance Center for denied permissions.

\section{LIMITED LICENSE}

The following terms and conditions apply only to specific license types:

15. Translation; This permission is granted for non-exclusive world English rights only unless your llcense was granted for translation rights. If you licensed translation rights you may only translate this content into the languages you requested. A professional translator must perform all iranslations and reproduce the content word for word preserving the integrity of the article. If this license is to re-use 1 or 2 figures then permission is granted for non-exciusive world rights in all languages.

16. Website: The following terms and conditions apply to electronic reserve and author websites:

Electronic reserve: If licensed material is to be pos ted to website, the web site is to be password-protected and made available only to bona fide students registered on a relevant course if:

This license was made in connection with a course.

This permission is granted for 1 year only, You may obtain a license for future website posting,

Al content posted to the web site must maintain the copyright information llne on the bottom of each image.

A hyper-text must be included to the Homepage of the joumal from which you are licensing at

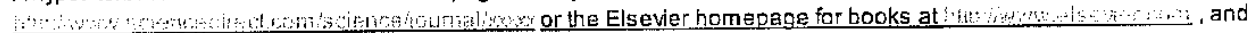

Central Storage: This Ilcense does not include permission for a scanned version of the material to be stored in a centra repository such as that provided by Heron/XanEdu.

17. Author website for journals with the following additional clauses:

Al content posted to the web site must maintain the copyright Information llne on the bottom of each image, and the permission granted is limited to the personal version of your paper. You are not allowed to download and post the publis hed electronic version of your article (whether PDF or HTML, proof or final version), nor may you scan the printed edition to create an electronic version. A hyper-text must be included to the Homepage of the journal from which you are

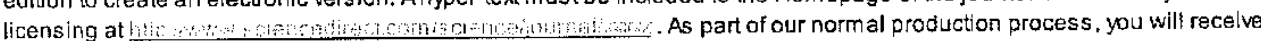
an e-mail notice when your article appears on Elsevier's online service SclenceDirect (www.sciencedirect.com). That email will include the article's Digital Object Identifier (DOI). This number provides the electronic link to the publis hed article and should be included in the posting of your personal version. We ask that you wait until you receive this e-mail and have the DOI to do any posting.

Central Storage: This license does not Include permission for a scanned version of the material to be stored in a central repository such as that provided by Heron/XanEdu.

18. Author website for books with the following additional clauses:

Authors are permitted to place a brief summary of their work online only.

A hyper-text must be included to the Elsevier homepage at hto linglts must maintain the copyright information line on the bottom of each image. You are not allowed to download and post the published electronic version of your chapter, nor may you scan the printed edition to create an electronic version.

Central Storage: This license does not include permission for a scanned version of the material to be stored in a central repository such as that provided by Heron/XanEdu.

19. Website (regular and for author): A hyper-text must be included to the Homepage of the journal from which you are licensing at or for books to the Elsevier homepage at http:/Www.elsevier.com

20. Thesis/Dissertation: If your license is for use in a thes/s/dissertation your thesis may be submitted to your institution in either print or electronic form. Should your thesis be published commercially, please reapply for permiss ion. These requirements include permission for the Library and Archives of Canada to supply single copies, on demand, of the complete thesis and include permission for UMl to supply single copies, on demand, of the complete thesis. Should your thes is be published commercially, please reapply for permlssion.

21. Other Conditions:

v1.6 
If you would like to pay for this license now, please remit this license along with your payment made payable to "COPYRGGI CLEARANCE CENTER" otherwise you will be invoiced within 48 hours of the flcense date. Payment should be in the form of a check or money order referencing your account number and this invoice number RLNK500869904. Once you receive your invoice for this order, you may pay your invoice by credit card. Please follow instructions provided at that time.

Make Payment To:

Copyright Clearance Center

Dept 001

P.O. Box 843006

Boston, MA 02284-3006

For suggestions or comments regarding this order, contact Rights Link Customer Support:

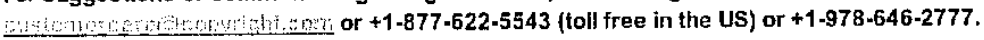

Gratis licenses (referencing $\$ 0$ in the Total field) are free. Please retain this printable license for your reference. No payment is required. 